\title{
SOLID-PHASE EXTRACTION OF BISPHENOL A IN WATER USING CARBON NANOTUBE ENVELOPES
}

\author{
By \\ Jenna Davies, B.Sc. \\ A thesis submitted to the Faculty of Graduate Studies and Research in partial fulfillment \\ of the requirements for the degree of \\ Master of Science \\ Department of Chemistry \\ Carleton University \\ Ottawa, Ontario \\ December 2010 \\ (C) copyright \\ 2010 Jenna Davies
}




\section{Library and Archives \\ Canada}

Published Heritage

Branch

395 Wellington Street Ottawa ON K1A ON4 Canada
Bibliotheque et

Archives Canada

Direction du

Patrimoine de l'édition

395 , rue Wellington

Ottawa ON K1A ON4

Canada
Yourfile Votre référence

ISBN: 978-0-494-79613-9

Our file Notre référence

ISBN: 978-0-494-79613-9
NOTICE:

The author has granted a nonexclusive license allowing Library and Archives Canada to reproduce, publish, archive, preserve, conserve, communicate to the public by telecommunication or on the Internet, loan, distribute and sell theses worldwide, for commercial or noncommercial purposes, in microform, paper, electronic and/or any other formats.

The author retains copyright ownership and moral rights in this thesis. Neither the thesis nor substantial extracts from it may be printed or otherwise reproduced without the author's permission.

\section{AVIS:}

L'auteur a accordé une licence non exclusive permettant à la Bibliothèque et Archives Canada de reproduire, publier, archiver, sauvegarder, conserver, transmettre au public par télécommunication ou par l'Internet, prêter, distribuer et vendre des thèses partout dans le monde, à des fins commerciales ou autres, sur support microforme, papier, électronique et/ou autres formats.

L'auteur conserve la propriété du droit d'auteur et des droits moraux qui protège cette thèse. $\mathrm{Ni}$ la thèse ni des extraits substantiels de celle-ci ne doivent être imprimés ou autrement reproduits sans son autorisation.
In compliance with the Canadian Privacy Act some supporting forms may have been removed from this thesis.

While these forms may be included in the document page count, their removal does not represent any loss of content from the thesis.
Conformément à la loi canadienne sur la protection de la vie privée, quelques formulaires secondaires ont été enlevés de cette thèse.

Bien que ces formulaires aient inclus dans la pagination, il n'y aura aucun contenu manquant.

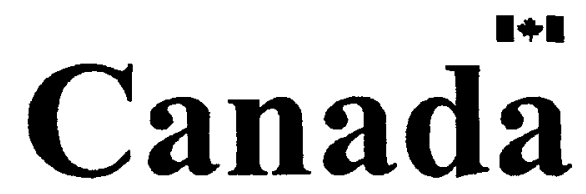




\begin{abstract}
Solid-phase extraction (SPE) of bisphenol A (BPA) from aqueous solution using a novel carbon nanotube (CNT) device has been investigated. Multi-walled carbon nanotubes (MWCNTs) were sandwiched between two sealed pieces of porous membrane. The final device resembled a commercial teabag. The SPE device, termed a CNT envelope, was prepared using polypropylene (PP) with pore diameter $0.2 \mu \mathrm{m}$ (denoted as PP-CNT envelope) and polyvinylidene fluoride (PVDF) with pore diameter $0.1 \mu \mathrm{m}$ (PVDF-CNT envelope). The device was immersed in aqueous BPA solution during adsorption and in methanol during desorption. The amount of BPA extracted by the CNT envelope was determined by HPLC with fluorescence detection.
\end{abstract}

BPA recovery from aqueous solution using PP-CNT envelopes was poor. To improve extraction efficiency, the device was conditioned in organic solvents prior to extraction; however the introduction of an organic phase significantly complicated the extraction system.

PVDF-CNT envelopes made were successful at extracting BPA from aqueous solution. Insignificant analyte carryover was observed between extractions and a single PVDFCNT envelope was used for nine successive BPA extractions from aqueous solution. BPA recovery using PVDF-CNT envelopes was determined to be $\sim 55 \%$ at equilibrium. The adsorption rate was examined and the length of time required to reach equilibrium, at least 9.25 hours, and was attributed to slow BPA diffusion through the membrane. 
BPA extraction was examined at different temperatures and the amount of BPA extracted increased at higher temperatures. The thermodynamic parameters of the overall system of moving BPA from water to the CNT surface were determined from a van Hoff plot. $\Delta \mathrm{H}^{\circ}{ }_{(\mathrm{rxn})}$ and $\Delta \mathrm{S}^{\circ}{ }_{(\mathrm{rxn})}$ were determined to be $37 \mathrm{~kJ} / \mathrm{mol}$ and $200 \mathrm{~J} / \mathrm{K} \cdot \mathrm{mol}$, respectively.

Adsorption using CNT envelopes resembles solid-phase microextraction (SPME).

Calculation of partition coefficients indicated BPA adsorption is an equilibrium extraction over a wide concentration and sample volume range. At low concentrations and small sample volumes, adsorption approached exhaustive extraction as is observed in SPME. The limit of detection of the method was determined to be $0.5 \mathrm{ng} / \mathrm{mL}$.

Single and multi-walled CNTs (SWCNTs and MWCNTs, respectively) were oxidized in dilute $\mathrm{HNO}_{3}$ to functionalize the CNT surfaces. BPA recovery decreased dramatically when the CNT envelope was prepared with oxidized nanotubes since the concentration of water molecules solvating the CNT surface increased with more polar groups. A comparison of extraction efficiency using CNTs with different diameters indicated BPA adsorption is most favourable on CNTs with smallest diameters.

BPA extraction from aqueous solution in polycarbonate baby bottles was examined using PVDF-CNT envelopes. BPA leaching to aqueous solution was examined at elevated temperatures in a microwave and oven, and at room temperature at extremely basic $\mathrm{pH}$. The amount of BPA extracted was dependent on the extraction conditions, although the mass of BPA extracted from every bottle was lower than the no observed adverse effect level (NOAEL) for BPA exposure. 


\section{ACKNOWLEDGEMENTS}

To Bob, thank you for giving me the opportunity to work in your lab all these years. I am so thankful for the experience and the knowledge I have gained. Thank you for your endless patience, generosity and support. Thank you for everything you have taught me about HPLC and GC-MS and for answering all of my questions, even if I'd already asked them before. You are an excellent supervisor, I am so appreciative of your guidance and I will be very sad to leave your lab.

To Steve Tremblay and coworkers at the Science Technology Center at Carleton University, thank-you for designing and fabricating the CNT envelope apparatus.

To Mohamed Abdel Salam, thank you for your friendship, support and for providing the surface area measurements and TEM images.

To Jim Logan, this work would not have been possible without your suggestions of how to suspend the teabags. Thank you for providing, fixing, and calibrating the many stir plates I needed. Thank you for somehow keeping the HPLC computer in working order!! To say thank you is not enough to show how much I appreciate the little things you have done to help me over the past 2.5 years.

To Jianqun Wang thank you for teaching me how to use the SEM and for helping me with all the imaging. 
To Tony O'Neil and Keith Bourque, thank you for your help designing the teabags in the early stages to the project, before I had the stainless steel pieces to make them myself. Further thanks to Tony for lending me the heat gun over and over again.

To Mastaneh, Elena, Graham and Daniel, thank you for all of the lab equipment you lent me when I needed it.

To Trevor and Jeab Porter, and Craig Nichols, a big thank you for taking the time to find baby bottles for me while travelling in Asia and for actually making room for them in your suitcases!

To Anatoli Ianoul, thank you for also providing me with baby bottles.

To Kamal, thank you for everything you taught me about making polymer films. Even though that work didn't make it into my thesis I am grateful for the time you spent teaching and helping me to make CNT polymer disks.

To Tony and Dave McMullin for driving me to and from school during the bus strike. Without your help, it would have been almost impossible for me to get to Carleton that winter.

To Farah, even though we only spent a short time together, I really enjoyed your presence in the lab. Thank you for being so understanding of the messy state I've left the lab in these past couple months. To Leda, thank you for all the little stories you entertained me with and thank you for all your help and support. And to Steve, I have thoroughly 
enjoyed becoming friends with you over the past year. Thank you for always smiling and bringing such positive energy to the lab. I have lost count of the number of laughs and jokes we have shared together. Thank you for all your help and support in the lab as well.

To the large number of friends who have helped, encouraged and supported me throughout this degree, thank you so much! Life is better spent with good friends!

And last but certainly not least, to Mom, Dad, Kyle and Austin. Thank you for all the love and support you have given me over the years and continue to give me every day. Thank you for being so understanding of the time and energy I needed to dedicate to this project. Thank you for always being there, I would not be here today if it wasn't for you. And with particular respect to my thesis, Dad thank you so much for all the time you have spent with me to create this document and for showing me all the things you can do in Word. And of course Dad, one of the biggest thank you's goes to you for all the time you spent with me taking the photographs in this document. They are perfect and hopefully one day I'll be as good a photographer as you. 


\section{TABLE OF CONTENTS}

1 INTRODUCTION........................................................................................................ 1

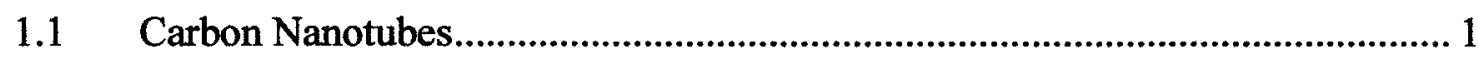

1.1.1 Introduction and History ........................................................................ 1

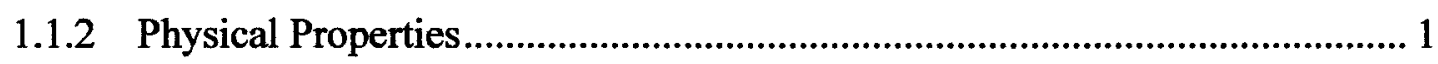

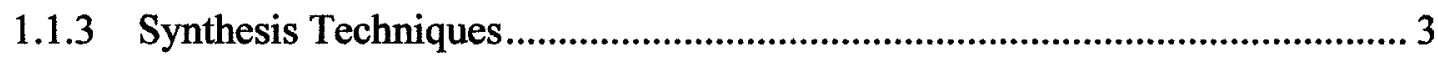

1.1.4 Oxidation and Functionalization............................................................ 4

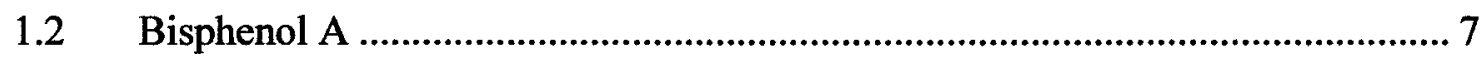

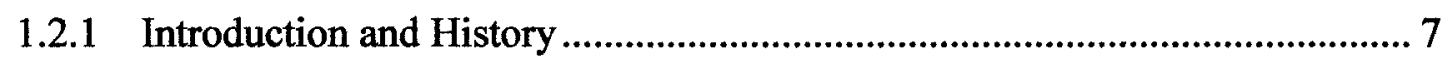

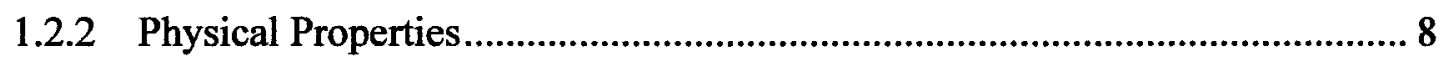

1.2.3 Effects on the Environment ....................................................................... 8

1.2.4 Health Effects and Toxicity ……............................................................. 10

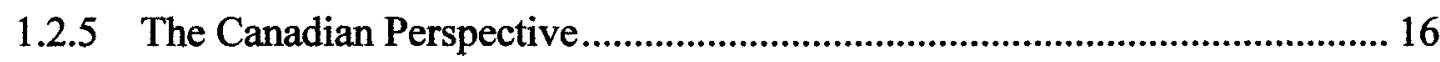

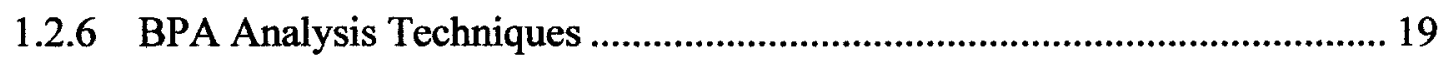

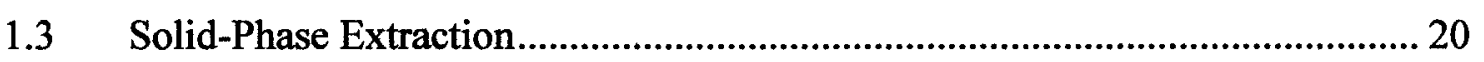


1.3.1 Solid-Phase Micro-Extraction

1.3.2 SPE and SPME Using Carbon Nanotubes. 24

1.3.3 BPA Analysis Using Carbon Nanotubes ........................................... 26

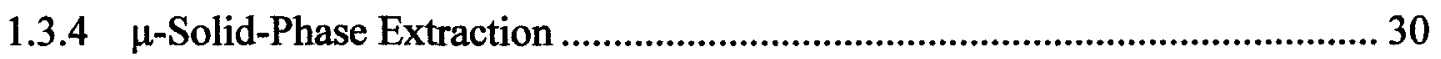

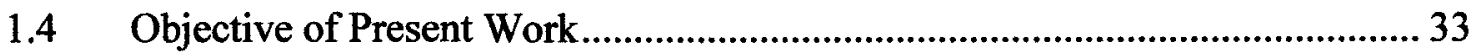

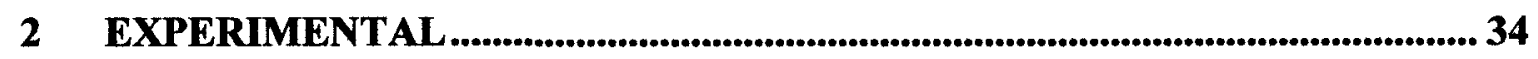

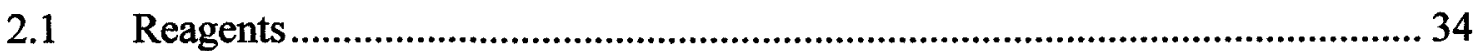

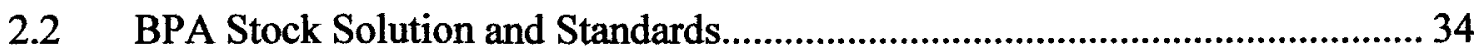

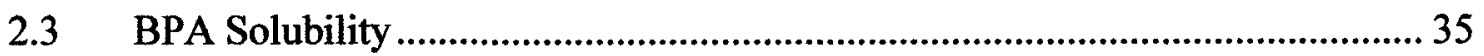

2.4 Preparation and Characterization of Pristine and Oxidized CNTs ................. 36

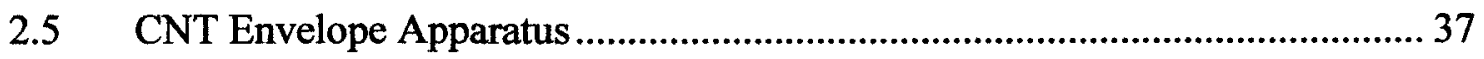

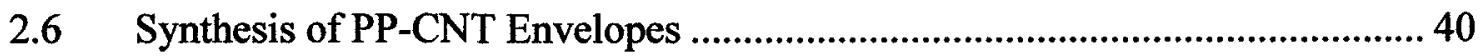

2.7 Initial BPA Extraction Using PP-CNT Envelopes...................................... 47

2.8 PP-CNT Envelope Conditioning Prior to Adsorption ................................ 47

2.8.1 Dipping PP-CNT Envelope in an Organic Solvent.................................... 48 
2.8.2 Soaking PP-CNT Envelope in an Organic Solvent. 48

2.8.3 Dipping the Rolled PP-CNT Envelope in an Organic Solvent 48

2.8.4 Sonicating the PP-CNT Envelope in an Organic Solvent. 48

2.8.5 Change in Mass of Conditioned PP-CNT Envelope over Time 48

2.9 Synthesis of PVDF-CNT Envelopes............................................................... 49

2.10 Initial Extraction of BPA Using PVDF-CNT Envelope ................................55

2.11 BPA Adsorption to PVDF-CNT Envelopes …............................................56

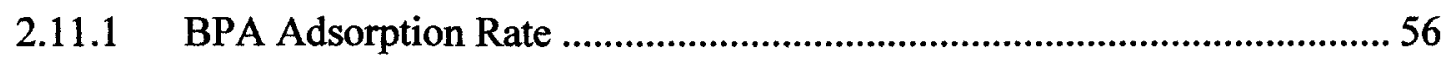

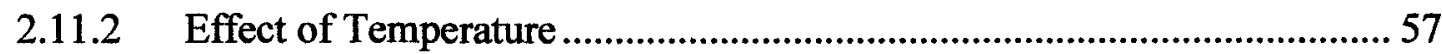

2.11.3 Effect of Concentration of Aqueous BPA solution...................................57

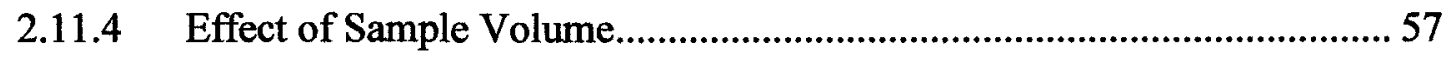

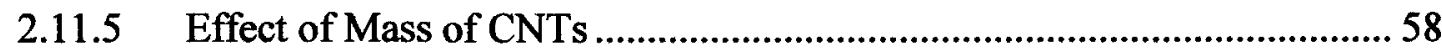

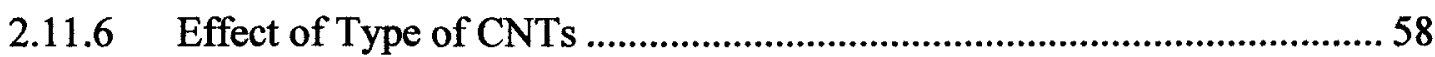

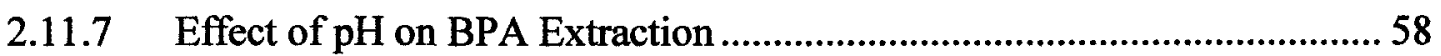

2.12 BPA Desorption to PVDF-CNT Envelopes..................................................58 
2.12.2 Desorption Solvent Volume.................................................................5

2.12.3 Effect of Desorption Solvent .............................................................. 59

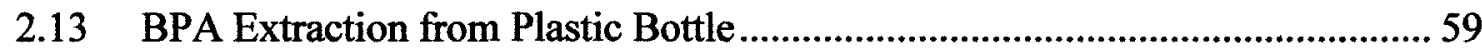

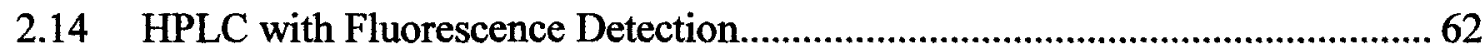

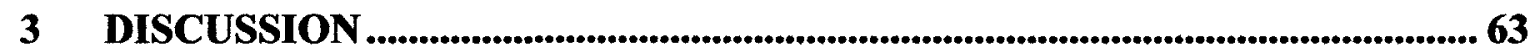

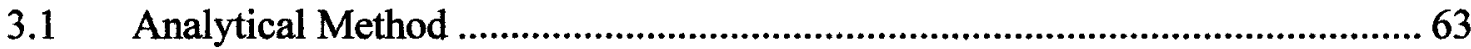

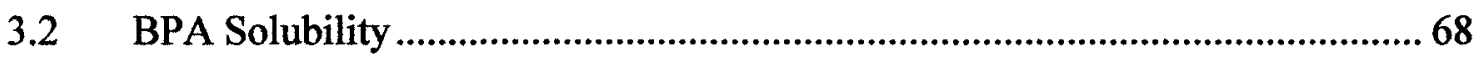

3.3 Carbon Nanotube Characterization................................................................. 74

3.3.1 Characterization of Polymer Membranes ....................................................... 83

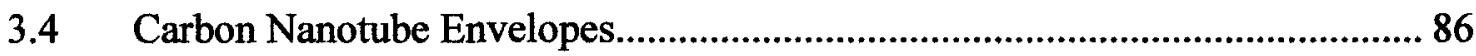

3.5 Overview of BPA extraction using CNT envelopes ..........................................91

3.6 Initial BPA Extraction Using PP-CNT Envelopes...........................................93

$3.7 \quad$ PP-CNT Envelope Conditioning................................................................... 98

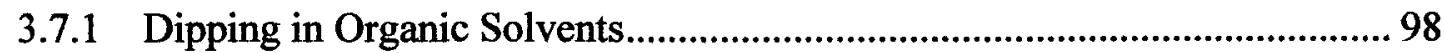


3.8 Initial BPA Extraction Using PVDF-CNT Envelopes

3.9 BPA Adsorption to PVDF-CNT Envelopes 130

3.9.1 BPA Adsorption Rate on PVDF-CNT Envelopes

3.9.1.1 BPA Adsorption with Stirring 131

3.9.1.2 BPA Adsorption with Sonication. 137

3.9.1.3 BPA Adsorption using PVDF-CNT Envelopes made from Membranes with Larger Pore Diameter. 139

3.9.1.4 Adsorption without stirring 141

3.9.2 Effect of Temperature 143

3.9.3 Effect of Sample Size and Concentration 148

3.9.4 Effect Mass of Carbon Nanotubes 156

3.9.4.1 Effect of Carbon Nanotube Functionality and Dimension 157

3.9.5 Effect of $\mathrm{pH}$ 161

3.10 BPA Desorption from PVDF-CNT envelopes. 163

3.10.1 Desorption Rate 165

3.10.2 Desorption Solvent Volume 167 


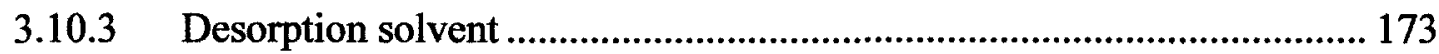

3.11 BPA Extraction from Baby Bottles Using PVDF-CNT Envelopes................ 176

4 CONCLUSION

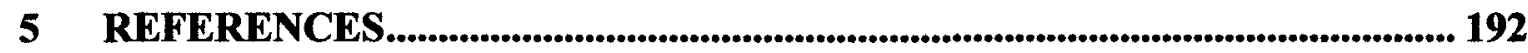




\section{LIST OF FIGURES}

Figure 1- 1 Schematic of a pristine carbon nanotube at the molecular level

2

Figure 1- 2 Oxidation of a pristine carbon nanotube in acid produces oxygen-containing groups at the CNT surface.

Figure 1- 3 Molecular structure of bisphenol A (BPA) 7

Figure 1- 4 BPA degrades in the environment to produce p-hydroxyacetophenone (pHAP), p-hydroxybenzaldehyde (p-HBAL),and 2,3-bis-(4-hydroxyphenyl)-1.2propanediol (Tetraol-IV) which are degraded further to produce p-hydroxybenzoic acid (p-HBA) $[24,25]$.

Figure 1- 5 Results of the urinary BPA concentration in the Canadian population, as determined from the survey Canadian Health Measures Survey (CHMS).

Figure 2-1 CNT envelope apparatus 39

Figure 2-2 The bottom PP punch is used to cut a disk from a sheet of polypropylene by pushing the punch into the membrane and turning clockwise. 42

Figure 2-3 The large (diameter: $5 \mathrm{~cm}$ ) and small (diameter: $2.25 \mathrm{~cm}$ ) polypropylene disks cut using the bottom and top PP punches, respectively. 42 
Figure 2-4 The large PP disk is placed on the die block (with indent side facing up) and held in place with the centering jig.

Figure 2-5 The forming tool is guided through the opening in the centering jig and pressed firmly on the large PP disk to create an indent. 43

Figure 2-6 The indent in the large PP disk created by the forming tool.

Figure 2-7 The centering tool is inserted into the opening in the centering jig and CNTs are transferred to the surface of the large PP disk through the hollow core of the centering jig. 44

Figure 2-8 The centering tool is removed and the small PP disk is gently placed on top of the CNTs through the opening of the centering jig 45

Figure 2-9 The sealer tool is heated to approximately $210^{\circ} \mathrm{C}$ using a heat gun. 45

Figure 2-10 The heated sealer tool is guided through the opening of the centering jig and pushed firmly down to create a seal between the small and large PP disks. 46

Figure 2-11 A PP-CNT envelope (shown left) and a PP-CNT envelope with excess membrane cut away from the seal (shown right). 46

Figure 2-12 A polyvinylidene fluoride disk is placed on a Millipore filter support and the centering tool is placed on top so that the opening of the tool is approximately in the 
center of the membrane. CNTs are introduced to the membrane through the hollow core of the centering tool.

Figure 2-13 Methanol is introduced through the hollow core of the centering tool to wet the CNTs. 52

Figure 2-14 The PVDF disk with CNTs collected in the center, is placed on the die block with indent side down. A second PVDF disk is placed over top of the disk containing the CNTs, guided by the centering jig. 53

Figure 2-15 A PVDF-CNT envelope (shown left) and a PVDF-CNT envelope with excess membrane cut away from the seal (shown right). 54

Figure 2-16 Open and closed twist-lock cable fasteners (shown left and center, respectively) and a piece of Teflon tubing with a slit cut half-way through the tube (shown right). Teflon-coated wire was threaded through the opening at the top of the closed twist-lock and through the core of the Teflon tube to suspend the CNT envelopes.

Figure 2-17 The baby bottles used for practical application of BPA extraction from aqueous solution using PVDF-CNT envelopes. From left: A1, A2 (behind A1), B1, C1, D1 (orange), D2 (purple, behind D1), E1, F1, G1, H1, H2 and H3 (both behind H1).... 60 65 
Figure 3-2 HPLC chromatogram of a $1 \mathrm{mg} / \mathrm{mL}$ BPA in methanol standard 65

Figure 3-3 HPLC chromatogram of a $100 \mathrm{ng} / \mathrm{mL}$ BPA in water standard 66

Figure 3-4 HPLC chromatogram of a water solution after BPA extraction using a CNT envelope 66

Figure 3-5 Calibration curve of BPA in methanol (red) and BPA in water (blue). 67

Figure 3-6 Concentration of BPA in aqueous solution over a wide range of temperature and $\mathrm{pH}$ conditions. 70

Figure 3-7 Concentration of BPA in organic solvents ranging in polarity. 71

Figure 3-8 Comparsion of BPA solubility to dipole moment, which reflects the degree of polar character the solvent has. 73

Figure 3-9 TEM image of SWCNTs 75

Figure 3-10 TEM image of long MWCNTs with a diameter of $10 \mathrm{~nm}$ as stated by the

manufacturer. 76

Figure 3-11 Infrared spectra of pristine (blue) and oxidized (red) SWCNTs. 77

Figure 3-12 Infrared spectra of pristine (blue) and oxidized (red) MWCNTs. 78

Figure 3-13 SEM image of pristine SWCNTs. 80

Figure 3-14 SEM image of oxidized SWCNTs 80 
Figure 3-15 SEM image of pristine MWCNTs

Figure 3-16 SEM image of oxidized MWCNTs.

Figure 3-17 Comarison of aqueous suspensions of pristine and oxidized SWCNTs (left pair) and pristine and oxidized MWCNTs (right pair). 83

Figure 3-18 SEM image of the polypropylene membrane, with a pore diameter of $0.2 \mu \mathrm{m}$, as reported by the manufacturer, Membrana $\mathrm{GmbH}$. 84

Figure 3-19 SEM image of the polyvinylidene fluoride membrane, with a pore diameter of $0.1 \mu \mathrm{m}$, as stated by the manufacturer, Millipore.

Figure 3-20 SEM image of the polyvinylidene fluoride membrane, with a pore diameter of $0.65 \mu \mathrm{m}$, as stated by the manufacturer, Millipore. .85

Figure 3-21 A PP-CNT envelope immersed in toluene to better illustrate what a CNT envelope looks like on the inside. 91

Figure 3-22 Comparison of BPA recovery between an empty PP envelope and PP--CNT envelope when the envelopes were placed in water directly for BPA extraction. 96

Figure 3-23 A schematic of possible interactions among water, BPA, CNTs and the polypropylene membrane during extraction. Repulsion between water and the hydrophobic membrane likely created a space in the pores of the membrane preventing 
water diffusion through the membrane and thus preventing BPA adsorption on the CNTs.

Figure 3-24 BPA recoveries when the PP devices (empty and filled) were conditioned by dipping in acetone prior to use. 100

Figure 3-25 BPA recoveries when the PP devices (empty and filled) were conditioned by soaking in acetone overnight prior to use. 101

Figure 3-26 BPA recoveries when the PP devices (empty and filled) were conditioned by dipping in methanol prior to use. 102

Figure 3-27 BPA recoveries when the PP devices (empty and filled) were conditioned by soaking in methanol overnight prior to use

Figure 3-28 BPA recoveries when the PP devices (empty and filled) were conditioned by dipping in dichloromethane prior to use. 105

Figure 3-29 BPA recoveries when the PP devices (empty and filled) were conditioned by sonication in acetone prior to use. 108

Figure 3-30 Time required for empty and filled PP envelopes to completely fill with solvent during condtitioning. 109

Figure 3-31 BPA recoveries when the rolled PP devices (empty and filled) were conditioned by dipping in acetone prior to use 112 
Figure 3-32 BPA recoveries when the rolled PP devices (empty and filled) were conditioned by dipping in methanol prior to use.

Figure 3-33 A schematic of the cross-section of a PP-CNT envelope when a conditioning solvent has been introduced to the extraction system. If the solvent is miscible with water, mixing will likely occur both inside and outside the PP-CNT envelope.

Figure 3-34 BPA extraction using envelopes prepared from the polyvinylidene fluoride membrane. Extraction efficiencies are compared for empty envelopes and envelopes filled with carbon nanotubes.

Figure 3-35 Extraction efficiencies from several extractions are compared to show the uncertainty in the amount of BPA recovered from one PVDF-CNT envelope to the next.

Figure 3-36 BPA recoveries when the intial extraction experiment was repeated using PVDF-CNT envelopes to determine if the other objects present in the system participated in extraction. 122

Figure 3-37 The amount of BPA adsorbed to the twist-lock fastener was determined when the PVDF-CNT envelope and twist-lock were separated for desorption during extraction

Figure 3- 38 PVDF-CNT envelopes suspended in water using a twist-lock fastener (shown left) and a piece of Teflon tubing (shown right). 125 
Figure 3-39 The extraction capability of the Teflon tube apparatus was explored before using it to suspend PVDF-CNT envelopes to ensure the problems associated with twistlock fasteners did not arise. 126

Figure 3-40 Two successive desorptions were performed on the same PVDF-CNT envelopes to determine if any BPA was retained in the envelope.

Figure 3-41 Reusability of a single PVDF-CNT envelope was explored over successive BPA extractions 129

Figure 3-42 Adsorption rate of BPA to CNT envelopes with stirring

Figure 3- 43 Dimensions of BPA 135

Figure 3-44 Partition coefficients corresponding to each point in equilibrium when the rate of BPA adsorption to CNTs was examined. 137

Figure 3-45 BPA extraction usinga single PVDF-CNT when adsoprtion was performed in a sonicator for 30 minutes.

Figure 3-46 BPA adsorption rate to PVDF-CNT envelopes that were prepared from a membrane with an effective pore diameter of $0.65 \mu \mathrm{m}$. 141

Figure 3-47 Rate of adsorption of BPA to PVDF-CNT envelopes without stirring....... 142

Figure 3-48 BPA recovery with changing adsorption temperature. 144

xiv 
Figure 3-49 van Hoff plot showing correlation between equilibrium constant and temperature.

Figure 3-50 BPA extraction from aqueous solution with changing concentration at constant sample volume. 150

Figure 3-51 Partition coefficient as a function of initial aqueous BPA concentration using PVDF-CNT envelopes.

Figure 3-52 BPA extraction from aqueous solution at constant concentration with changing sample volume.

Figure 3-53 Relationship between partition coefficient and sample volume. 155

Figure 3-54 BPA extraction from aqueous solution containing the same mass of BPA with changing sample volume. 156

Figure 3-55 BPA extraction using PVDF-CNT envelopes filled with varying masses of LMWCNTs

Figure 3-56 Comparison of BPA extraction using CNTs of different diameters (pristine single, double or multi-walled) and of different functionality (pristine or oxidized SWCNTs and MWCNTs) 159

Figure 3-57 Effect of carbon nanotube length on BPA recovery when the nanotube diameter is constant. 161 
Figure 3-58 Effect of $\mathrm{pH}$ of aqueous solution on BPA extraction using PVDF-CNT

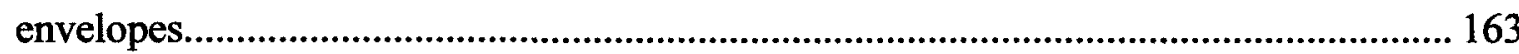

Figure 3-59 BPA desorption from PVDF-CNT envelopes with stirring. ......................... 166

Figure 3-60 BPA desoprtion from PVDF-CNT envelopes without stirring. 167

Figure 3-61 Volume of methanol required for complete BPA desorption with stirring. 169

Figure 3-62 Two methods of suspending PVDF-CNT envelopes: on left, a PVDF-CNT envelope suspended in $15 \mathrm{~mL}$ methanol and on right, a PVDF-CNTenvelope could be suspended in $1 \mathrm{~mL}$ of methanol.

Figure 3-63 Volume of methanol required for complete BPA desorption without stirring.

Figure 3-64 Comparison of desorption solvents for BPA elution from the PVDF-CNT envelopes 176

Figure 3-65 Baby bottles used for practical application of BPA extraction from aqueous solution using PVDF-CNT envelopes. From left: A1, A2 (behind A1), B1, C1, D1 (orange), D2 (purple, behind D1), E1, F1, G1, H1, H2 and H3 (both behind H1) 178

Figure 3-66 Standard addition curves of old bottle A1 and new bottle B1 to determine the amount of BPA leached from the bottles into aqueous solution 180 
Figure 3-67 Chromatogram showing the presence of another analyte overlapping with BPA when the BPA extract from a baby bottle was anayzed by HPLC. The composition of the mobile phase was $90 \% \mathrm{MeOH}: 10 \% \mathrm{dH}_{2} \mathrm{O}$ to start.

Figure 3-68 Chromatogram showing the separation between BPA and any other analytes when the composition of the mobile phase was changed to $50 \% \mathrm{MeOH}: 50 \% \mathrm{dH} 2 \mathrm{O}$ to start.

Figure 3-69 Concentration of BPA leached from old bottles A1 and A2 after several successive extractions.

Figure 3-70 Concentration of BPA leached into water from old bottles D1 and D2 after several successive extractions. 185 


\section{LIST OF TABLES}

Table 3-1 Comparsion between the solubility of BPA and dipole moment of the solvent

Table 3-2 Specific surface area measurements of the carbon nanotubes used in this work.

Table 3-3 Volume of solvent needed to fill a PP-CNT envelope with acetone, hexane or methanol 110

Table 3-4 Mass of BPA extracted from each baby bottle. 187

xviii 


\section{INTRODUCTION}

\subsection{Carbon Nanotubes}

\subsubsection{Introduction and History}

In 1991, Iijima [1] discovered graphitic carbon needles deposited on the cathode during the synthesis of fullerenes by the arc-discharge evaporation process. These carbon nanostructures, later termed carbon nanotubes, were 4-300 $\mathrm{nm}$ in diameter and up to 1 $\mu \mathrm{m}$ in length. Electron micrographs showed evidence of multiple walls within the nanotubes, with a number of walls recorded up to 50 sheets. Two years later, Iijima [2] reported the discovery of single -wall carbon nanotubes found in soot-like deposits in a carbon-arc chamber. Unlike other bulk or nano-scale materials, carbon nanotubes are characterized by their unique structural, electrical, chemical and mechanical properties [3]. It has been reported that carbon nanotubes have a tensile strength 100 times greater than steel, and a thermal conductivity better than diamond. They have an electrical conductivity similar to copper but have the capacity to carry significantly higher currents [4]. In addition, they also have the largest elastic modulus of any known material and very high surface areas [3]. Since their discovery, research involving carbon nanotubes has grown significantly due to the exploitation of their novel properties and their potential for a wide range of applications [5].

\subsubsection{Physical Properties}

A carbon nanotube (CNT) is best described as a graphene sheet rolled along a lattice vector to form a seamless cylindrical hollow nanostructure, as shown in Figure 1- 1 
$[3,4,6]$. Each carbon atom is bonded to three neighbouring carbon atoms through $\mathrm{sp}^{2}$ hybridization [4]. The ends of carbon nanotubes are generally capped with an arc surface of pentagons and heptagons, resembling part of a fullerene structure. The presence of pentagons and heptagons affects the cylindricity of the CNT at the tip, which causes local distortions in the caps and affects the localized properties of the ends [6].

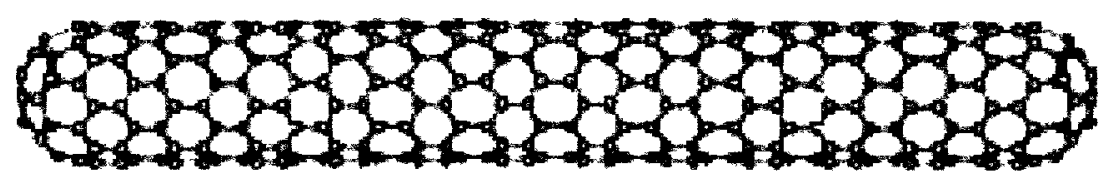

Figure 1-1 Schematic of a pristine carbon nanotube at the molecular level

Carbon nanotubes may be layered with one or more cylindrical graphene sheets inside one another $[3,4,6]$ and are identified as single, double, or multi-walled depending on the number of layers $(1,2$ or more than 2 , respectively). Single-walled carbon nanotubes (SWCNTs) typically have diameters of $0.2-2 \mathrm{~nm}$, while multi-walled carbon nanotubes (MWCNTs) have outer diameters of 2-100 nm [3], and 0.3-0.4 $\mathrm{nm}$ spacing between layers [6]. In a MWCNT the individual planes have no set configuration relative to one another, unlike the planes of graphite which are stacked in an ordered fashion relative to one another [6]. The delocalized aromaticity of CNTs allows them to $\pi-\pi$ stack with other aromatics. Due to their extremely narrow diameter compared to their long lengths, CNTs have a very high aspect ratio (on the order of 100-1000) and can essentially be thought of as a one-dimensional nanostructure [6,7]. 


\subsubsection{Synthesis Techniques}

Three methods are commonly employed for the synthesis of carbon nanotubes: arc discharge, laser ablation and chemical vapour deposition. CNTs were first produced by the arc discharge method [8]. In this synthesis, two carbon rods are placed end-to-end and an arc plasma is generated between them by applying a dc current in an inert gas atmosphere at low pressure $[4,6]$. A deposit grows on the cathode while the anode is consumed. Generally, the deposit has a soft fibrous core and a hard outer shell formed of fused CNTs and nanoparticles. The fibrous core is made up of bundles of smaller fibers that are in fact bundles of even smaller fibers [6]. CNTs are only formed at the cathode when the current is flowing since they have not been observed anywhere else in the chamber. Several gases have been reported for use during the arc discharge process including helium [8,9], argon [], methane [10] and hydrogen [11]. When the carbon anode contains a metal catalyst (Fe, Co, etc), SWCNTs are generated on the carbon cathode instead of MWCNTs [2].

Despite the growth of SWCNTs using the carbon arc method, laser ablation or the laser furnace method is commonly used for the production of SWCNTs. A carbon composite containing catalytic metals is placed in the center of a quartz tube furnace and exposed to a laser beam of $\mathrm{CO}_{2}[4]$. SWCNTs are formed when the composite is vapourized in a high-temperature, argon atmosphere. SWCNT diameters can be altered with changes to the furnace temperature, flow rate and catalytic metals [12]. Laser ablation affords high quality SWCNTs with regard to diameter and growth control [4]. 
Chemical vapour deposition (CVD) allows control of alignment and location of CNTs during synthesis compared to arc-discharge and laser ablation [4]. In CVD, hydrocarbon vapour is passed through a tube furnace containing catalyst particles at temperatures high enough to decompose the hydrocarbon. As the hydrocarbon vapour passes over the catalyst, CNTs grow and can be collected when the system is cooled to room temperature. The type of hydrocarbon, type of catalyst and growth temperature will all affect CNT production [4]. Unlike arc discharge and laser ablation, CVD can render large scale nanotube production or production of a single CNT [13].

\subsubsection{Oxidation and Functionalization}

CNTs possess the strong ability for chemical modification compared to planar carbon structures, such as graphite, due to their high degree of curvature. Functionalization at the capped ends is most energetically favourable compared to the sides, due to the degree of curvature in two dimensions as a result of the presence of pentagons and heptagons at the caps $[14,15]$. Although CNTs are generally regarded as having a highly uniform surface, their structure is not completely homogenous and often contains defects formed during synthesis. It has been reported that $1-3 \%$ of carbon atoms are located in a defect site in a CNT. One of the most common defect sites, known as the Stone-Wales defect, is composed of a small cluster of five and seven-membered rings which distorts the curvature of the nanotube surface along its side. Any deformations along the sidewall will increase the reactivity of the defect site [16].

Methodologies for nanotube functionalization can be divided into two groups [3]. The first involves CNT oxidation in the presence of strong acids. During the oxidative 
process, pristine (pure) CNTs are sonicated or refluxed in mixtures of dilute or concentrated nitric and sulfuric acid, depending on the degree of functionalization desired $[3,17]$. These highly oxidative conditions lead to the formation of holes in the sidewalls and opening of the end caps, resulting in a shortening of CNT lengths $[3,4,14]$. The final products are really CNT fragments, with reported lengths of 100-300 nm. Nanotube shortening can be minimized under less vigorous conditions (ie. refluxing in diluted acids) in order to retain the electronic and mechanical properties of the CNTs [14]. Upon oxidation, the ends and sidewalls of a CNT will be decorated with a high density of oxygen-containing functionalities, mostly carboxyl groups, as demonstrated in Figure 12. Alcohols, esters and ketones have also been observed [18]. The presence of oxygenated functional groups provides sites for further CNT modification. Through the creation of ester and amide bonds, nanotubes can be modified with a broad range of moieties, including biologically active species, polymers, metals and other nanoparticles [14]. 


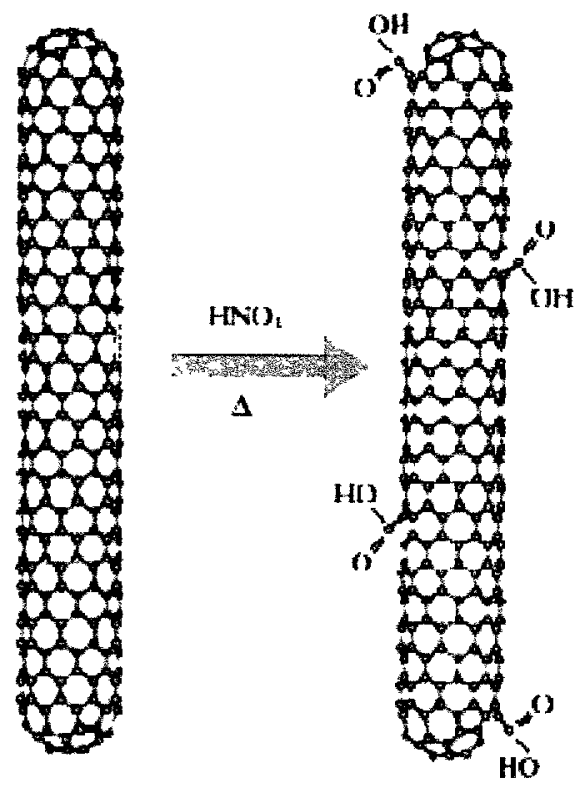

Figure 1-2 Oxidation of a pristine carbon nanotube in acid produces oxygen-containing groups at the CNT surface.

The second functionalization process involves addition reactions directly at the CNT surface [14]. These reactions usually involve heat, although not all mechanisms are understood. Under these conditions the desired functional group is coupled directly to the $\pi$-conjugated framework of the CNTs. The degree of functionalization increases with smaller tube diameters. Upon modification through addition reactions, CNTs can be further functionalized via substitution reactions. CNT functionalization with carboxyl groups or other functionalities prevents CNTs from aggregating into bundles due to a reduction in the van der Waals forces between nanotubes and to increased solvation. This allows for the separation of CNT bundles into individual nanotubes. Consequently, the solubility of carbon nanotubes in aqueous or organic media can be greatly enhanced $[4,14]$. 


\subsection{Bisphenol A}

\subsubsection{Introduction and History}

Bisphenol A or BPA, is the common name for the synthetic chemical 2,2-(4,4'dihydroxydiphenyl) propane $[19,20]$. The chemical structure of BPA is shown in Figure 1- 3 .

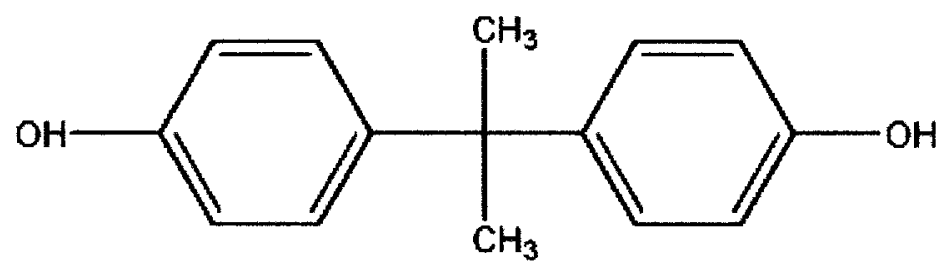

Figure 1- 3 Molecular structure of bisphenol A (BPA).

There are a few different methods of manufacturing BPA, however, generally synthesis involves condensing phenol with acetone under low $\mathrm{pH}$ and high temperature conditions, in the presence of catalysts. Distillation technology is used to purify the crude BPA and then the purified product is filtered and dried [20]. There is no evidence that suggests any natural occurrence of BPA, or any formation of BPA as a breakdown product from a more persistent organic contaminant. In the early 1950s, BPA was first used as a monomer to synthesize polycarbonate plastics and resins [21], and today almost all manufactured BPA is used as an intermediate in the production of these products, as well as flame retardants and other specialty products. Other final products containing BPA include adhesives, powder paints, automotive lenses, protective window glazing, building materials, compact disks, optical lenses, thermal paper and paper coating. BPA is incorporated into protective coatings, including dental coatings and sealants. It has been 
included as a developer in dyes and has been used for the encapsulation of electrical and electronic parts [20].

\subsubsection{Physical Properties}

BPA exists as a white solid in the shape of crystals, prills or flakes, under ambient conditions $[19,20]$. It is a relatively hydrophobic compound with a water solubility of 120-300 mg/L [20]. Due to its two OH groups, BPA has two dissociation constants, pKa 9.59 and 11.30 , and thus is more water soluble at alkaline $\mathrm{pH}$ [19]. It will not appreciably deprotonate at environmental $\mathrm{pH}$ levels (generally $\mathrm{pH} 7$ and lower). The airwater partition coefficient, $\mathrm{K}_{\mathrm{AW}}$, of BPA is very low $\left(10^{-9}\right)$ and thus BPA is not likely to evaporate from aqueous media [19]. The octanol-water coefficient, $\mathrm{K}_{\mathrm{OW}}$ is used to describe analyte partitioning between solvent and water. The log $\mathrm{K}_{\mathrm{OW}}$ of BPA is 3.323.64 which suggests BPA will easily partition to an aqueous phase [19]. The octanol-air partition coefficient, $\mathrm{K}_{\mathrm{OA}}$, is $2.6 \times 10^{12}$. This coefficient is used to describe analyte partitioning between air and soil. The $\mathrm{K}_{\mathrm{OA}}$ for BPA suggests that gaseous BPA will sorb to soil surfaces (soils, vegetation, aerosols, etc.) quite strongly [19].

\subsubsection{Effects on the Environment}

BPA is one of the highest volume chemicals produced worldwide. Each year over 6 billion pounds are produced and a portion of BPA produced during synthesis can be released to air, surface waters, or wastewaters, during manufacturing, processing and transportation $[22,23]$. In addition, wastes created during the manufacture of other products containing BPA will also contribute to BPA emissions to the environment. In the environment, more than $98 \%$ of BPA will partition to surface water (30\%) and soil 
(68\%), and thus BPA will be primarily lost from these media [19]. Once released to the environment, BPA will be dispersed and degraded by biotic and abiotic fate processes, including biodegradation, adsorption to suspended solids and sediments, and potentially photodegradation. In the atmosphere, BPA is likely lost by reaction with hydroxyl radicals [20].

Aerobic biodegradation of BPA in water and soil is the most common loss process and occurs via complicated metabolic pathways, as shown in Figure 1- 4. Approximately $80 \%$ of BPA undergoes cleavage to produce p-hydroxyacetophenone (p-HAP) and phydroxybenzaldehyde (p-HBAL) which are degraded further to produce $\mathrm{p}$ hydroxybenzoic acid (p-HBA). The remaining $20 \%$ is converted to $2,3-$ bis-(4hydroxyphenyl)-1.2-propanediol (Tetraol-IV) and then to bis(4-hydroxyphenyl)-1propanol $[24,25]$. The reported half-life of BPA in surface water is very short (3-6 days) and is not dependent on certain experimental parameters, including location, sampling site, sediment addition or chemical concentration [26]. In soil, BPA half-life was reported as less than 3 days [19]. The half-life criteria for persistent chemicals as outlined by Environment Canada are 2 days in air, 6 months in water and soil and 1 year in sediments. BPA is not considered to be persistent in the environment when its halflives in environmental media (air, water, soil and sediment) are assessed against the halflife criteria for persistent chemicals outlined by Environment Canada [19, 27]. When emitted to the atmosphere, BPA is not expected to travel far from point sources, however, in surface waters, BPA may travel hundreds of kilometers downstream from the emission source [19]. 


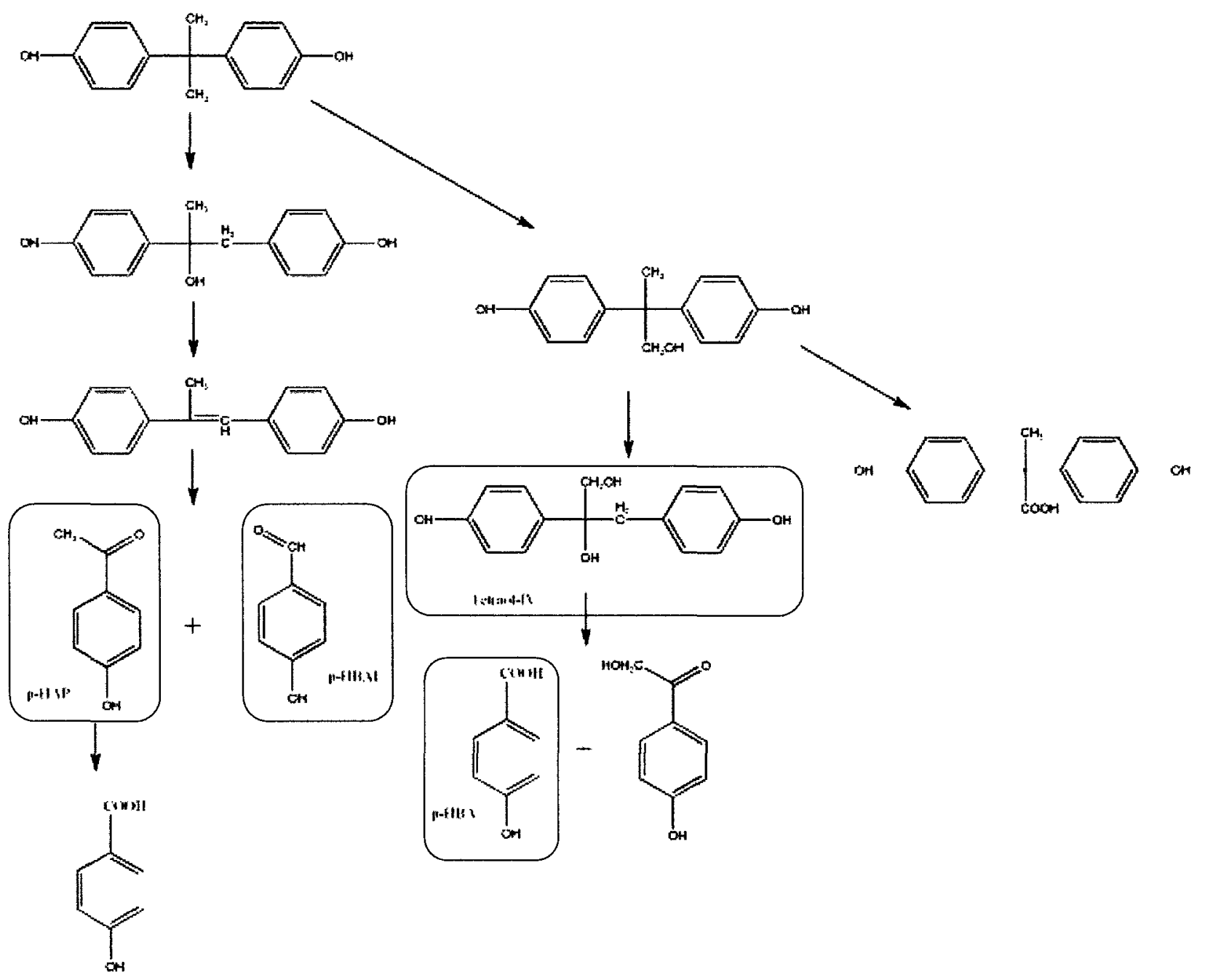

Figure 1- 4 BPA degrades in the environment to produce $\mathrm{p}$-hydroxyacetophenone (pHAP), p-hydroxybenzaldehyde (p-HBAL), and 2,3-bis-(4-hydroxyphenyl)-1.2propanediol (Tetraol-IV) which are degraded further to produce $p$-hydroxybenzoic acid $(\mathrm{p}-\mathrm{HBA})[24,25]$.

\subsubsection{Health Effects and Toxicity}

Humans are exposed to BPA mainly through environmental sources (air, water, soil), especially close to emission point sources [20]. Exposure is widespread, studies from Canada, the US and Europe all report detectable levels of BPA in more than $90 \%$ of the population $[28,29]$. The population that are employed in BPA manufacturing facilities, or 
facilities that use BPA as an intermediate in manufacturing other products are of greater risk through occupational exposure [20]. BPA has also been reported to migrate from epoxy resins lining metal cans and beverage containers, and from polycarbonate storage containers, especially at elevated temperatures $[22,30]$. The number of studies estimating total BPA exposure to humans is minimal. Kang et al. [31] estimated daily human intake of BPA from both environmental (air, water, soil) and food (coated metal can surfaces, polycarbonate containers) contamination to be less than $1 \mu \mathrm{g} / \mathrm{kg} \bullet$ body weight (bw)/day. Alternatively, BPA exposure from food sources alone was estimated to be 0.48-1.6 $\mu \mathrm{g} / \mathrm{kg} \cdot \mathrm{bw} /$ day by the European Commission's Scientific Committee on Food [32]. According to the IUPAC Compendium of Chemical Technology [33], the no observed adverse effect level (NOAEL) of a target organism is defined as the highest amount or concentration of a substance which causes no detectable adverse alteration of morphology, functional ability, growth, development, or life span of the organism under set exposure conditions. NOAELs are used for regulatory purposes and are determined by experiment or observation using lowest observed adverse effect levels (LOAEL). Two multi generation studies examining BPA effects on rats and mice reported a LOAEL of $50 \mathrm{mg} / \mathrm{kg}$ • bw/day $[34,35]$ which was used to establish a regulatory NOAEL of 5 $\mathrm{mg} / \mathrm{kg} \cdot \mathrm{bw} /$ day for BPA in Europe and the US [36,37]. Based on the NOAEL and LOAEL established from the rat and mice studies, Health Canada established a provisional tolerable daily intake (TDI) for BPA of $25 \mu \mathrm{g} / \mathrm{kg} \bullet \mathrm{bw} / \mathrm{day}$ [62]. BPA elicits cellular responses in the body because it fits in the estrogen receptor (ER) binding pocket and thus can bind to both estrogen receptors, ER $\alpha$ and ER $\beta$ [22]. BPA 
also interacts with the androgen receptor (AR) [30]. These interactions interrupt the normal cell-signaling pathways of these hormone receptors. Thus, BPA is considered to be a xenoestrogen (an industrial compound that exhibits estrogenic effects) or an environmental estrogen [22]. However, BPA has a significantly lower affinity for nuclear ERs compared to estradiol (approximately 10000 times weaker) [38], and consequently has been labeled a weak environmental estrogen [39,40]. BPA is metabolized in the liver by two enzymes that are capable of metabolizing xenoestrogens and other toxic substances. EDP-glucuronosyltransferases are responsible for the glucuronidation of BPA to BPA glucuronide and phenol sulfotransferases conjugate BPA to BPA sulfate [22]. Both of these metabolites exhibit little to no estrogenic activity [41]. Toxicity, potential adverse effects and conclusions about the health risks associated with BPA have been widely investigated [30], however findings are contradictory and inconclusive, and no consensus has been made regarding what BPA exposure levels cause a health risk and what the health risks are. BPA risk assessment has proven to be a particularly controversial topic among toxicologists and other experts. In particular, the "low-dose" effects of BPA have been one of the biggest sources of dispute among scientists, and are responsible for much of the BPA risk assessment controversy. Despite having a NOAEL in the $\mathrm{mg} / \mathrm{kg} \bullet \mathrm{bw} /$ day range, BPA exposure at $\mu \mathrm{g} / \mathrm{kg} \bullet \mathrm{bw} /$ day levels has been reported to have effects on laboratory animals [30].

Low-dose exposures to laboratory animals have been reported to have effects on the development and function of male and female reproductive organs, and neurobehavioural development [30]. Typically when the toxicity of a substance is examined, correlation is 
expected between high doses and greater adverse effects. In other words, effects that are observed at high doses are not expected at low-doses. However, in the case of some natural and synthetic hormones, biphasic dose responses have been observed for certain endpoints. A biphasic dose response curve is U-shaped or inverted U-shaped and illustrates that hormone signaling may activate more than one pathway [22]. BPA exposure to control animals has been reported to elicit biphasic dose responses in behaviors, protein expression, embryonic development and organ size [42]. Consequently, some studies designed to examine significant changes to morphology or development following exposure to high doses of BPA may not reveal subtle changes that are still of significant concern [43]. As such, hypotheses about the safety of lowdoses of BPA may not be valid if conclusions drawn from high dose exposure do not necessarily predict the endpoints observed at low-doses [44]. Since BPA has been shown to initiate cellular response at concentrations well below the levels where BPA is expected to bind to estrogens receptors, it is conceivable that the accepted NOAEL for BPA is not a reflection of its true potency [22].

In addition to low-dose effects, exposure to test animals during various stages of development has been the cause for some debate. Many BPA exposure studies fail to examine exposure throughout all levels of development, since most studies have been focused on exposure levels during adulthood. Throughout development, ERs will be present in different types of cells and diverse effects on the same organ could occur during different life stages when exposed to low-doses of BPA. Furthermore, effects to low-doses of BPA during fetal development may not be apparent until later stages in life 
[22]. It has been concluded that BPA crosses the maternal-fetal placental barrier because notably high levels of BPA have been measured in umbilical cord blood, fetal plasma and the serum of pregnant women [45]. Vom Saal and Moyer [46] predicted that the perinatal period of development (just before and after birth) is the time when an organism is most sensitive to BPA effects. This prediction was supported by studies examining the amount of BPA needed to induce a uterotrophic response in female rodents. It was observed that levels of $\mu \mathrm{g} / \mathrm{kg} \bullet \mathrm{bw} / \mathrm{day}$ were necessary for response in adult rodents while levels of only $\mathrm{ng} / \mathrm{kg} \cdot \mathrm{bw} /$ day invoked a change in the development of estrogen-target organs later in life, when fetuses were exposed in utero $[47,48]$. As previously mentioned, BPA is metabolized in the body by liver enzymes, however the amount of BPA metabolized has not been quantified and may differ between sexes. Infant rodents have limited ability to metabolize BPA into an inactive form, regardless of BPA administration route [49]. Thus, human fetuses and infants could suffer from the same challenge. Studies of BPA metabolism in rodents found that enzymes in the colon and digestive tract successfully deconjugated conjugated BPA [50]. This suggests that the possible deconjugation of conjugated BPA into a biologically active form by other body tissues may exist. In the human body, glucuronidase production in the digestive tract is increased from infancy until adult levels are reached at age 4. As a result, infants may also deconjugate conjugated BPA during digestion [51].

Furthermore, disparity between BPA exposure levels and measured human concentrations is the cause for some contest among researchers. Studies claiming rapid BPA metabolism rates and excretion from the body indicate that BPA should be 
undetectable in human samples when compared with estimated exposure levels [52]. However, analysis of blood, tissue and urine samples indicates definite, widespread occurrence of BPA [53,54]. These findings suggest that BPA exposures may not all be accounted for or the estimated daily intake is too low. Conversely, the currently accepted metabolic model for BPA breakdown may be inaccurate or BPA may bioaccumulate in the body [45].

Other sources of contention that contribute to the BPA toxicity dispute are focused on the methods used to perform experiments that draw conclusions about the health effects of BPA. There is debate about whether the use of Good Laboratory Practices (GLP) should be essential criterion for accepted exposure studies and risk assessments, and whether those low-dose studies already published that failed to meet GLP standards are viable [22]. Particularly, contamination from collection materials or during storage is cause for concern. Deliberations about the most appropriate exposure route for laboratory animals are also a cause of significant objection. Several studies demonstrate differences in pharmacokinetics depending on exposure through oral, intravenous, or subcutaneous avenues $[45,52,55]$. In addition, some findings suggest BPA metabolism differs between rodents and primates, and consequently the application of rodent models to human metabolism of BPA is under question [22].

Despite the controversy surrounding BPA toxicity, some expert panels have identified positive correlations and potential adverse effects of BPA. In rodents, low doses of BPA led to developmental changes, weight changes and size differences in male and female reproductive organs $[56,57,58]$. Cells in the mammary glands, brain, and skeletal and 
cardiovascular systems have also been associated with BPA exposure [59]. In the rodent brain, BPA has also been shown to alter sexually dimorphic pathways; differences in sexually dimorphic behaviours became indistinguishable after exposure. Aggressive behaviour in adult rodents $[56,60]$ and defeminization of female rodents has also been observed [59]. Furthermore, rats prenatally exposed to BPA displayed sensitivities to carcinomas in the mammary gland later in life. Since BPA levels are detectable in more than $90 \%$ of the human population and human exposure is likely chronic, not acute, no identifiable control group exists. In women, associations have been made between endometrial hyperplasia, occurrence of miscarriages, sterility, ovarian dysfunction and BPA concentrations in the blood [22]. An increased risk for type II diabetes and cardiovascular disease has been correlated with BPA levels in urine. In addition, adverse effects in experimental animals to low doses of BPA have been related to recent trends in human diseases. These include an increase in prostate and breast cancer, uro-genital abnormalities in male babies and early onset of puberty in girls. A link has also been made between neurobehavioural problems such as attention deficit hyperactivity disorder (ADHD) in humans and effects observed in laboratory animals [21].

\subsubsection{The Canadian Perspective}

In the human population, fetuses and infants are most at risk to BPA exposure, and it has been hypothesized that adverse effects to BPA exposure in fetuses and infants may not be apparent until significantly later in life $[21,22,30]$. Furthermore, it is unethical to initiate human studies on this part of the population. The Canadian government was the first country to complete a risk assessment of BPA in consultation with industry and other 
stake holders. They identified that the main source of BPA exposure for newborns and infants is through leaching from polycarbonate baby bottles, especially when exposed to elevated temperatures, and migration into infant formula from the epoxy linings in metal cans. Although newborn and infant exposure to BPA was below levels that may pose a risk, Health Canada identified the gap between exposure and effect as not wide enough. Consequently, in April 2008, the Government of Canada was the first governmental body to take actions to reduce BPA exposure to newborns and infants by banning polycarbonate baby bottles. In addition, the government proposed to develop alternative food packaging and stringent migration targets for BPA in cans of infant formula. In October 2010, the Canadian Government added BPA to its list of chemical substances in the Chemical Environmental Protection Act, thereby formally designating BPA as a toxic substance, a decision the chemical industry fought strongly against. Days later, government also announced further legislation that would "require facilities to develop and implement plans to limit BPA releases to the environment and to submit ongoing progress reports" [62].

In August 2010, Statistics Canada released its findings from the Canadian Health Measures Survey (CHMS) [63] which was conducted in partnership with Health Canada and the Public Health Agency of Canada. The focus of the CHMS was to evaluate lead and BPA concentrations in the Canadian population from March 2007 to February 2009. People, aged 6 to 79, were surveyed at fifteen sites across the country, which consequently represented $96.3 \%$ of the population. The survey consisted of a face-toface interview and urine sampling from a mobile examination centre. 
Results from the survey indicated BPA exposure in Canada is widespread; $91 \%$ of the population aged 6-79 had detectable levels of BPA in their urine, with an average concentration of $1.16 \mu \mathrm{g} / \mathrm{L}$. The results were sorted according to age group and gender, as shown in Figure 1- 5.

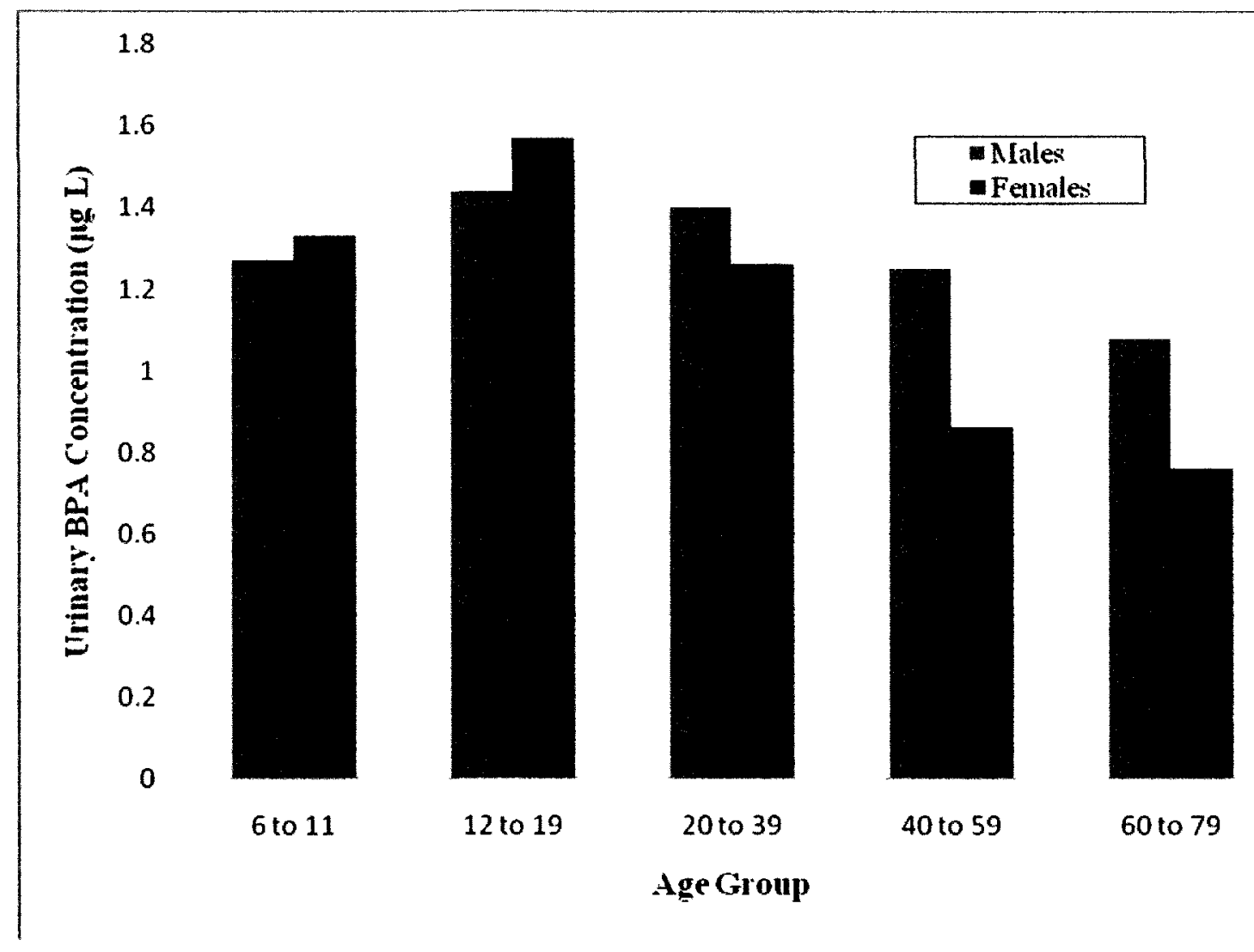

Figure 1- 5 Results of the urinary BPA concentration in the Canadian population, as determined from the survey Canadian Health Measures Survey (CHMS).

Overall, adolescents, aged 12-19 had the highest urinary BPA concentrations. Men, aged 40-79, had statistically higher levels compared to women in the same age group. Children may be more susceptible to higher BPA levels than adults due to their higher food intake compared to their body weight. Furthermore, children and adults may experiences differences in absorption, distribution, metabolism or excretion of BPA. 
Also, since BPA use has increased since the 1970 s, it is likely that the younger age groups would have been more exposed to BPA from the use of products containing the endocrine disruptor. Variation in BPA levels between genders may be the result of differences in exposure or in pharmocokinetic factors. The influence of household income, country of birth and BMI status were examined to see if they significantly impacted BPA concentration, however when the variables age, gender, and time of urine collection were held constant, no other factors were correlated with BPA levels.

The results from the CHMS were in agreement with the National Health and Nutrition Examination Survey (NHANES) from the U.S. [28] which reported detectable levels of BPA in $93 \%$ of the population, aged 6 or over. BPA was detected in $99 \%$ of the German population, aged 6 or over, according to the German Environmental Survey [29]. Although, the reasons for higher BPA levels in children compared to adults and men compared to women need to be better identified through additional research, this is the first national survey measuring BPA exposure to Canadians and it serves as a strong foundation to represent exposure levels to the Canadian population.

\subsubsection{BPA Analysis Techniques}

Separation and detection of BPA is mainly performed using GC/MS [64] or HPLC [65]. HPLC with fluorescence detection is an advantageous detection method because it does not require the derivatization steps necessary for GC/MS analysis. Typically, reversed phase $\mathrm{C}_{18}$ columns are used with mixtures of water, methanol and acetonitrile as the mobile phase. After excitation at $275 \mathrm{~nm}, \mathrm{BPA}$ has been found to exhibit fluorescence at 300-320nm [66]. HPLC-MS detection also avoids tedious derivatization work-ups and 
can be used for BPA detection. Electrospray ionization (ESI) and atmospheric pressure chemical ionization (APCI) are both used in negative mode for BPA analysis; however ESI is used more frequently as it provides better sensitivity. Regardless of the ion source used, the most abundant peak on an LC-MS spectrum of BPA will be $\mathrm{m} / \mathrm{z} 227,[\mathrm{M}-\mathrm{H}]$ " Other fragment ions observed include the additional loss of oxygen, $\mathrm{m} / \mathrm{z} 211$ or a methyl radical, $\mathrm{m} / \mathrm{z} 212$. LC coupled with electrochemical detection has also been reported [66]. Higher resolution and lower detection limits for BPA determination are afforded by GCMS analysis; however, derivatization steps are required prior to chromatographic detection. Silyation with BSTFA or acetylation using acetic anhydride or trifluoroacetic anhydride are the most widely used derivatization procedures. GC-MS with electron impact (EI) ionization is generally used for analysis. Peaks in the mass spectrum correspond to the parent ion, $\mathrm{m} / \mathrm{z} 228$ and the loss of a methyl group, $\mathrm{m} / \mathrm{z} 213$ [66]. Although chromatographic analysis of BPA is the most common analytical method, BPA determination using ELISA has also been reported [66]. Prior to separation, sample preparation techniques are almost always used to prepared sample for chromatographic detection. Solid-phase extraction is most widely used for BPA isolation, however liquidliquid extraction techniques have also been documented. Less common extraction methods, including microwave assisted extraction, pressurized liquid extraction, stir bar sorptive extraction and solid-phase micro-extraction have also been reported [66].

\subsection{Solid-Phase Extraction}

As highly sensitive chromatographic instruments evolved for the detection of contaminants in the environment, sample preparation and pre-concentration methods also 
emerged to cope with the demand for accurate and precise environmental analysis [67]. Liquid-liquid extraction (LLE) and solid-phase extraction (SPE) techniques were developed to prepare an environmental sample for analysis $[67,68]$. Solid-phase extraction involves the partitioning of compounds between two phases, one being solid, the other liquid or gas. Analytes are retained on the solid phase via adsorption to the surface since they will have a great affinity for the solid phase than the liquid or gas phase. Later, analytes will be eluted or desorbed from the solid sorbent if they have a greater affinity still for the solvent. SPE is advantageous compared to LLE for sample preparation due to its shorter times required, ability to handle small samples, use of small volumes of organic solvents, no formation of emulsions and can provide more reproducible results. In modern SPE procedures, the sorbent is passed into a cartridge or embedded in a disk. Aqueous samples containing the analyte (s) of interest are pumped or pulled through the SPE cartridge or disk are exposed to the sorbent surface [68]. Over time, several sorbents have been developed for SPE, including activated carbon, alumina, silica gel, magnesium silicate, and chemically bonded to silica and polymers [68].

\subsubsection{Solid-Phase Micro-Extraction}

In 1989, prior to the discovery and use of CNTs for SPE, a modification to classical SPE procedures was developed to address the need for a rapid, solvent-free, sensitive SPE technique for use in both laboratory and on-site analysis [69-71]. In this approach, sections of fused-silica optical fibers were coated with a liquid or solid polymeric phase [70]. The coated fiber was dipped in an aqueous solution containing the analytes of interest and then simply placed in a GC injector for analyte elution. Although the 
polymeric phase could be liquid or solid, the technique was named solid-phase microextraction (SPME), since the apparatus involved a stationary sorbent phase coated on a solid support [70]. Modern SPME apparatus resemble a modified syringe. A thin, cylindrical fused-silica fiber coated with thin films of the polymeric stationary phase is connected to a piece of stainless steel tubing, which is in turn connected to a specifically designed syringe-like instrument [69]. Control of the plunger movement allows fiber exposure during extraction and elution, and protection in the needle during storage and transportation [69]. Extraction is performed by immersing the coated fiber directly in a liquid sample, known as direct immersion SPME (DI-SPME) or by exposing the fiber to a vapour phase above a liquid or solid sample, referred to as head-space SPME (HSSPME) [69]. Analytes are desorbed from the fiber with an appropriate solvent or the fiber can be attached directly to GC or HPLC systems for solvent-free elution of analytes, and delivery directly to the chromatographic column. The type of fiber coating, choice of sampling mode, use of agitation conditions, internal temperature fiber temperature and external sampling temperature will all affect the efficiency of analyte extraction by SPME [71]. SPME techniques are advantageous compared to other pre-concentration methods because they are fast, portable, solvent-free, simple and easy to use [69-71].

Unlike exhaustive SPE procedures, SPME involves equilibrium partitioning to the sorbent surface. Using SPME, maximum extraction efficiency occurs at the partition equilibrium, the equilibrium reached between analytes partitioning to the sorptive fiber and left in the sampling media. When the fiber is coated in a liquid polymeric coating, the amount of analyte absorbed by the coating is directly proportional to its concentration 
in the sample. This relationship between amount of analyte absorbed, $n$, and sample concentration, $\mathrm{C}_{0}$, is modelled by:

$n=\frac{K_{f s} V_{f} C_{0} V_{s}}{K_{f s} V_{f}+V_{s}}$

Where $\mathrm{K}_{\mathrm{fs}}$ is the partition coefficient of analyte between the fiber coating and sample matrix, and $V_{f}$ and $V_{s}$ are the volumes of fiber coating and sample matrix, respectively $[72,73]$.

Analytes partitioning to the fiber from the sample matrix tends to be high since organic analytes generally have a strong affinity for the polymeric coating. As a result, good sensitivity is obtained due to a high concentrating effect by the fiber coating. Although the partition coefficients $\left(\mathrm{K}_{\mathrm{fs}}\right)$ will be large in SPME, partitioning is generally not significant enough to exhaustively extract most analytes [72].

SPME is often used for field sampling and when the sample volume is very large, $V_{f} \gg$ $\mathrm{K}_{\mathrm{fs}} \mathrm{V}_{\mathrm{s}}$ and the expression for amount of analyte absorbed can be simplified [72,73]:

$n=K_{f s} V_{f} C_{0}$

Since the amount of analyte extracted is independent of sample volume at large volumes, the fiber can be directly exposed to the sample matrix in the field, such as ambient air, water, rivers, lakes, etc, and sample collection and clean-up can be avoided. In other words, SPME integrates extraction, pre-concentration and introduction into chromatographic instruments into a single step [72,73]. 
At small sample volumes, however, the amount of analyte removed by the fiber is significant compared to the sample volume such that $V_{\mathrm{f}} \ll \mathrm{K}_{\mathrm{fs}} \mathrm{V}_{\mathrm{s}}$, and SPME is no longer an equilibrium extraction. When exhaustive extraction is achieved, owing to very small sample volumes, the amount of analyte removed is redefined as:

$n=V_{s} C_{0}$

This implies the fiber coating extracted all of the analytes in the sample matrix and thus the mass of analyte removed is easily calculated from the sample concentration and fiber coating $[72,73]$.

\subsubsection{SPE and SPME Using Carbon Nanotubes}

In 2001, Long et al [74] reported strong sorption of dioxins to the surface of MWCNTs. These pronounced interactions were attributed to the unique structure and electronic properties of CNTs. The interactions of dioxin to MWCNTs and activated carbon were compared, and the amount of dioxin adsorbed to the CNT surface was tens of orders of magnitude larger than that of activated carbon. The immense sorptive capability of CNTs was attributed to their high surface area and ability to $\pi-\pi$ stack with aromatic analytes. In addition to their unique structural and electrical characteristics, CNTs possess excellent thermal, mechanical and chemical stability. These attributes and expanding research about CNT functionalization make carbon nanotubes very attractive as potential SPE sorbents for both organic and inorganic compounds. A sample of the publications of CNTs as SPE sorbents are mentioned below. 
MWCNTs have been explored for removal of several pesticides from aqueous solution including DDT [75], atrazine and simazine [76], and diazinon [77]. Extraction efficiencies of MWCNTs outperformed $\mathrm{C}_{18}$ and graphitized carbon black when compared for removal of atrazine and simazine from tap and well water [78]. MWCNTs were also used as SPE sorbents for analysis of antidepressants in urine [79] and barbiturates in pork [80]. Pristine SWCNTs were explored for removal of tetracyclines from surface waters [81], while carboxylated SWCNTs were use for sorption of non-steroidal antiinflammatory drugs from urine [82]. Several phthalates in tap, river and sea water were extracted by MWCNTs with equal or greater extraction efficiencies than $\mathrm{C}_{8}, \mathrm{C}_{18}$, and polystyrene-divinylbenzene [84]. MWCNTs were also explored for extraction of many phenols, including 2,4-D, TCP and PCP from aqueous solutions. Salam and Burk [85] compared the extraction capabilities of pristine, oxidized, and MWCNTs functionalized with polyethylene glycol or octadecylamine for removal of TCP, PCP and two PCBs from river water. MWCNTs have also been applied as SPE sorbents for extraction of several inorganic ions from aqueous solution, including $\mathrm{Cd}^{2+}, \mathrm{Mn}^{2+}$, and $\mathrm{Ni}^{2+}[86]$ in lake water, $\mathrm{Cu}^{2+}[87], \mathrm{Pb}^{2+}[88], \mathrm{Hg}^{2+}[89]$, and $\mathrm{Co}^{2+}[90]$ in tap water and $\mathrm{Cr}^{3+}$ and $\mathrm{Cr}^{6+}[91]$ in river and wastewater. Carbon nanotubes have also been explored for use as coating on SPME fibers. SWCNTs were functionalized with hydroxyl-terminated silicone oil and use to prepare an SPME fiber through sol-gel techniques. The functionalized fiber was successfully used for HS-SPME of several polybrominated diphenyl ethers in reservoir and wastewater samples [92]. SWCNT-coatings have also demonstrated higher extraction efficiencies compared to commercial PDMS fibers for extraction of organochlorine pesticide in aqueous solution. Oxidized MWCNTs have been used to 
coat silica fibers prior to SPME detection of several phenols in aqueous solution [92]. A unique SPME approach involving CNTs was explored by Sae-Know et el. A removable capillary probe was packed with either SWNCTs or MWCNTs and attached to a syringe to create a novel SPME setup. An aqueous solution of 2-nitrophenol, 2,6-dichlorophenol and naphthalene was drawn into the probe for extraction. SWCNTs and MWCNTs containing hydroxyl and carboxylic groups improved analyte retention compared to their pristine counterparts [92].

\subsubsection{BPA Analysis Using Carbon Nanotubes}

Few studies have been published using CNTs as a sorbent for removal of BPA from aqueous matrices. Cai et al. [93] removed the packing from a $C_{18}$ cartridge and filled it with MWCNTs to use as an SPE method for the removal of BPA, 4-n-nonylphenol and 4tert-octylphenol from aqueous solution. Several extraction parameters, including sample volume, flow rate, eluent type and volume, and $\mathrm{pH}$ were examined. Recovery was essentially $100 \%$ for all three analytes for sample volumes of $100-750 \mathrm{~mL}$ and decreased slightly at $1000 \mathrm{~mL}$. The most effective eluent was determined to be methanol.

Recoveries of 4-n-nonylphenol and 4-tert-octylphenol were not significantly influenced by varying $\mathrm{pH}(\mathrm{pH} 3-11$ ), however for $\mathrm{BPA}$ an increase in $\mathrm{pH}$ above $\mathrm{pH} 8$ (above the first $\mathrm{pKa}$ ) resulted in a dramatic decrease in recovery. The limit of detection for BPA was determined to be $0.083 \mathrm{ng} / \mathrm{mL}$. Cartridge reusability was also examined, it was determined that carryover from one extraction to the next was negligible due to complete desorption. The MWCNT packed cartridge was compared to a $\mathrm{C}_{18}$ cartridge and an $\mathrm{XAD}-2$ copolymer cartridge. The XAD-2 copolymer cartridge was not as effective at 
extracting any of the analytes compared to the MWCNT packed cartridge, while the $\mathrm{C}_{18}$ cartridge displayed comparable extraction efficiency for the removal of the endocrine disruptors, except BPA.

The majority of SPE studies relating to the use of CNTs as adsorbents involve packing CNTs in an SPE cartridge. Niu et al. [84] investigated a method of preparing an SPE disk from SWCNTs. SWCNTs were suspended in sodium dodecyl sulfate and filtered through a qualitative filter paper under vacuum, creating a uniformly dispersed SWCNT filter. Aqueous solutions containing the model analytes (BPA, 4-nonylphenol, and 4-tertoctylphenol) were filtered through the SWCNT disk. Since the disks were quite thin (thickness: $0.25 \mathrm{~mm}$ ), they could be stacked for simultaneous extraction using multiple disks. It was found that stacking the extraction disks significantly increased recovery compared to single disk extraction. Extraction involving two disks, or double-disk (DD) extraction was found to have an equally high extraction capability as a MWCNT packed cartridge despite the significantly smaller amount of CNTs used to prepare the disk (60 mg SWCNTs versus 300-500 mg MWCNTs). The limit of detection of BPA using double-disk extraction was determined to be $7 \mathrm{ng} / \mathrm{L}$. When compared with a $\mathrm{C}_{18}$ disk and an activated carbon disk, the SWCNT disk displayed comparable extraction efficiencies with the $\mathrm{C}_{18}$ disk for the removal of phthalates and increased capabilities for the extraction of chlorophenols, except BPA. Furthermore, the DD disk was used to extract the aforementioned chlorophenols from real water samples.

Kuo [94] examined the use of pristine and modified CNTs for BPA removal from aqueous solution. Pristine CNTs were synthesized by CVD and then modified with 
$\mathrm{SOCl}_{2}$ and $\mathrm{NH}_{4} \mathrm{OH}$ by microwave assisted oxidation procedures to produce carboxyl and amine groups at the carbon nanotube surface, as confirmed by IR spectroscopy. For adsorption, pristine or modified CNTs were suspended in an aqueous solution containing BPA via shaking and then centrifuged and filtered for collection after extraction. Specific surface area measurements of the pristine and modified CNTs revealed that the CNT surface area increased with chemical modification. The pristine and modified CNTs proved successful for BPA removal from water over a wide temperature $\left(7-47^{\circ} \mathrm{C}\right)$ and $\mathrm{pH}(\mathrm{pH} 3-9)$ range, respectively, without a decrease in BPA recovery. The enthalpy $\left(\Delta \mathrm{H}^{\circ}\right)$ and entropy $\left(\Delta \mathrm{S}^{\circ}\right)$ change of the system were determined. The value of $\Delta \mathrm{H}^{\circ}(-$ $11.7 \mathrm{~kJ} / \mathrm{mol}$ ) revealed adsorption was exothermic, and suggested the interaction between BPA and CNTs during adsorption was physical, rather than chemical. The value of $\Delta \mathrm{S}^{\circ}$ (46.1 J/mol) indicated BPA was less ordered on the CNT surface compared to in aqueous solution. All values of $\Delta \mathrm{G}^{\circ}$ indicated BPA adsorption to the CNT surface was a spontaneous process.

Rastkari et al. [95] reported a solid-phase micro-extraction approach using SWCNTs for BPA pre-concentration from canned food. Oxidized SWCNTs were attached to a piece of stainless steel wire using an organic binder. The fiber was used for HS-SPME analysis of acylated bisphenol derivatives. The SWCNT-coated SPME fiber was extremely robust with a life span of over 150 uses. When compared to a commercial PDMS fiber, the SWCNT-coated fiber was found to be more sensitive and have better thermal stability for the determination of bisphenol $\mathrm{A}$ and bisphenol $\mathrm{F}$ in canned food. 
Li et al. [96] prepared a SWCNT-coated fiber for SPME analysis of BPA from aqueous samples. SWCNTs were oxidized in a mixture of concentrated acids and then suspended in DMF. Two Pt wires, used as an anode and a cathode, were immersed in the SWCNTs suspension, and a voltage was applied between them. SWCNTs were deposited on the Pt wire acting as an anode. This process, electrophoretic deposition, could be repeated for various amount of time to produce fibers with coatings of different thicknesses. The SWCNT coating did not strip off from the Pt wire substrate, nor swell in the presence of organic solvents, and had a high mechanical strength. Since both SWCNTs and the platinum wire were conductive, the SWCNT-coated fiber was conductive. In addition, the fiber was essentially unbreakable due to the durability of the Pt substrate. The SPME-coated fiber was used in direct immersion SPME sampling mode for the evaluation of six phenols; phenol, p-nitrophenol, m-methylphenol, 2-chlorophenol, 2,4dichlorophenol and BPA from aqueous solution, followed by HPLC-UV analysis. Extraction capabilities of the SWCNT-coated fiber were compared to a commercial poly(acrylate) (PA) fiber for analysis of the same group of phenols. The SWCNTprepared fiber was determined to have similar or superior extraction efficiencies compared to the PA fiber, likely due to the high surface area and the presence of oxygenated groups on the SWCNTs. Last, the SWCNT-coated fiber was successfully used for the analysis of the group of phenols from seawater and tap water matrices with limits of detection between 0.9 and $3.8 \mathrm{ng} / \mathrm{mL}$. 


\subsection{4 $\mu$-Solid-Phase Extraction}

The challenge of developing a practical SPE method using CNTs as the sorbent for the removal of contaminants from the environment has been explored by Basheer et al. [97]. The objective of their work was to develop a micro SPE procedure, alternative to MWCNT cartridges and SPME to remove organophosphorous pesticides (OPP) from sewage sludge samples. To create a $\mu$-SPE device, a porous polypropylene membrane was folded in half and heat-sealed around two of the remaining open edges. MWCNTs ( $\sim 6 \mathrm{mg})$ were introduced into the pocket through the open edge, which was thereafter sealed, immobilizing the MWCNTs within the protective membrane. The device was cleaned by ultrasonication in acetone for 10 minutes and then stored in acetone until use. During adsorption, the device was allowed to tumble freely in the sewage sludge sample, aided by magnetic stirring. After 30 minutes, the $\mu$-SPE device was removed and rinsed with ultrapure water. The organophosphorous pesticides were then desorbed from the device by ultrasonication in hexane. Extracts were analyzed by GC-MS.

Low wettability of the $\mu$-SPE device and the CNTs was observed, likely due to the hydrophobic nature of both the polypropylene membrane and the carbon nanotubes. Consequently, low extraction efficiencies and slow extraction rates were observed. To overcome this challenge, the $\mu$-SPE device was conditioned in several organic solvents (methanol, acetone, hexane, dichloromethane, and toluene) by submerging the device in the solvent for approximately 1 minute and then ultrasonicating the $\mu$-SPE device in water for 2 minutes. The polypropylene membrane and carbon nanotubes retained only trace volumes of the conditioning solvent. The conclusions drawn about the effect of 
organic conditioning solvent on extraction efficiency were inconclusive and contradictory. First, the authors stated that when comparing extraction efficiencies between devices conditioned with polar and non-polar solvents, it was found that nonpolar solvents (toluene and dichloromethane) gave higher results than polar solvents. However, they later indicated that the use of a conditioning solvent did not influence extraction efficiency. The authors indicated that organic conditioning solvents had been used in the past to improve the wettability of carbon nanotubes, for their use as an SPE sorbent [93]. Extraction was also performed using an empty polypropylene device to ensure the membrane itself did not participate in the adsorption process.

The extraction parameters adsorption time, desorption time, temperature, $\mathrm{pH}$, sample volume and desorption solvent were optimized. The highest extraction efficiency was obtained after 30 minutes for all OPPs, and complete desorption from the envelope was observed after sonication for 30 minutes. The desorption solvent used was not reported, however in a study of ideal desorption solvent, dichloromethane and acetone obtained the highest extraction efficiencies. Extraction was performed from $25-80^{\circ} \mathrm{C}$ and recovery increased slightly at $80^{\circ} \mathrm{C}$ for almost all OPPs. Extraction was also examined in sample volumes from $5-25 \mathrm{~mL}$ and efficiency was highest at $20 \mathrm{~mL}$ for almost all OPPs. Recovery of all OPPs increased from $\mathrm{pH} \mathrm{2-8}$ and decreased at $\mathrm{pH} 10$ and higher. The limit of detection using the $\mu-\mathrm{SPE}$ device was determined to be $1-7 \mathrm{pg} / \mathrm{g}$. After desorbing the OPPs from the $\mu$-SPE device, a second desorption was performed and no OPPs were detected. Thus, it was determined that no analyte carryover occurred from one extraction to another. As a result, experiments were performed repeatedly with a single device and 
it was observed the extraction efficiency was not compromised for up to 30 extractions. In addition, the extraction capability of the $\mu$-SPE device was compared to hollow fiber membrane protected SPME (HFM-SPME) and head space SPME (HS-SPME) for OPP removal, and the $\mu$-SPE device was determined to be the most efficient extraction method.

$\mu$-SPE was also explored for the removal of two acidic drugs, ketoprofen and ibuprofen, from wastewater [98]. $\mu$-SPE devices were prepared in the same fashion, except the mass of CNTs was increased to $20 \mathrm{mg}$, and experiments were performed under similar conditions, however some unique extraction parameters were explored. First, the sorbents $\mathrm{C}_{18}, \mathrm{C}_{2}$, Carbograph, HayeSep A, HayeSep B, and MWCNTs were all used to fill the $\mu$-SPE devices. The study indicated that $C_{18}$ was the most efficient sorbent for both drugs. This was attributed to high surface area and high electrostatic interaction with both drugs. The authors stated that the electrostatic interaction between the carbon nanotubes and acidic drugs was not as strong compared to $\mathrm{C}_{18}$, resulting in a lower extraction efficiency. Second, several different membranes (polypropylene, polysulfone, GV Durapore, polycarbonate) were explored for the synthesis of $\mu$-SPE devices containing $\mathrm{C}_{18}$. Polypropylene was found to be the most compatible with the conditioning solvent, methanol, resulting in the highest extraction rates compared to the other membranes. Last, extraction using multiple $\mu$-SPE devices (1-4) was also examined. It was found that as the number of $\mu$-SPE devices increased, extraction efficiency increased as well, however little to no increase was observed after extraction with two $\mu$-SPE devices. 


\subsection{Objective of Present Work}

The objective of this work is to develop a novel SPE device, similar in design and dimension to the $\mu$-SPE device developed by Basheer et al. [97], for the extraction of BPA from aqueous solution using carbon nanotubes. The device, termed a CNT envelope, will be used to study the fundamentals of BPA adsorption to CNTs; including the adsorption rate, thermodynamics of the process and BPA partitioning from water to the CNTs. Extraction parameters; including adsorption and desorption time, temperature, sample volume, concentration and $\mathrm{pH}$, and mass and type of CNTs will be optimized to develop an analytical method for BPA removal using the envelopes. Last, the CNT envelopes will be evaluated as SPE adsorbents for analysis of water in contact with polycarbonate baby bottles. 


\section{EXPERIMENTAL}

\subsection{Reagents}

Pristine "long" single-walled (diameter: $<2 \mathrm{~nm}$ ), double-walled (diameter: $<5 \mathrm{~nm}$ ), multiwalled (diameter: $10 \mathrm{~nm}$ and diameter: $10-20 \mathrm{~nm}$ ) carbon nanotubes, with tube length 5-15 $\mu \mathrm{m}$ were all purchased from Shenzhen Nanotech Port. Co. Ltd. (Shenzhen, China). Pristine "short" multi-walled (diameter: 10-20 nm) carbon nanotubes with length 1-2 $\mu \mathrm{m}$ were also purchased from Shenzhen Nanotech Port. Co. Ltd. (Shenzhen, China). Accurel® PP 2E HF (R/P) polypropylene membrane (pore diameter: $0.2 \mu \mathrm{m}$, thickness: $167 \mu \mathrm{m}$ ) was purchased from Membrana GmBH (Wuppertal, Germany). Durapore® (polyvinylidene fluoride) membrane filters (pore diameter: $0.1 \mu \mathrm{m}$, thickness: $125 \mu \mathrm{m}$ ) were purchased from Millipore (Billerica, MA, USA). Voltrex twist-lock cable fasteners were supplied by Newark (Palatine, IL, USA). Bisphenol A ( $\geq 99 \%$ ) was also supplied by Sigma-Aldrich. Optima LC/MS grade methanol, optima grade hexane and spectro grade chloroform were purchased from Fisher Scientific. Spectro grade acetone, toluene, dichloromethane, ethyl acetate and HPLC grade acetonitrile were supplied by Caledon Laboratories. A Milli-Q water purification system was used to prepare ultrapure water. Compressed air was supplied by Praxair.

\subsection{BPA Stock Solution and Standards}

A $100 \mathrm{mg} / \mathrm{L}$ stock solution of BPA in methanol was prepared by dissolving $50 \mathrm{mg}$ of BPA in $500 \mathrm{~mL}$ of methanol. The solution was shaken by hand until all BPA visibly dissolved. BPA standards in either water or methanol were diluted from the $100 \mathrm{mg} / \mathrm{L}$ 
BPA in methanol stock solution. Using the serial dilution method, standards of $1,5,10$, $50,100,250,500$, and $1000 \mathrm{ng} / \mathrm{mL}$ were prepared. All standards were analyzed using HPLC with fluorescence detection to determine the linear dynamic range of BPA and produce a calibration curve.

\subsection{BPA Solubility}

To determine the solubility of BPA in water over a wide $\mathrm{pH}$ range, a Fisher Accumet Model $210 \mathrm{pH}$ meter was used to accurately measure the $\mathrm{pH}$ of deionized water which was spiked with a small volume of either dilute $\mathrm{NaOH}$ or $\mathrm{HCl}$. The water was combined with solid BPA in a glass centrifuge vial and the vial was shaken vigorously to promote dissolution. BPA was continuously added until it would no longer dissolve and a visible amount of solid BPA could be seen in the vial. The vial was placed in a Neslab RTE-8 refrigerated circulating bath and the temperature was maintained at $25 \pm 0.5^{\circ} \mathrm{C}$. Periodically, $10 \mu \mathrm{L}$ of the supernatant was removed using a $10 \mu \mathrm{L}$ syringe and diluted with methanol for HPLC analysis. The concentration of the supernatant was determined from a calibration curve of methanol standards. The centrifuge vial was shaken after every $10 \mu \mathrm{L}$ of supernatant was removed. This procedure was repeated until the concentration of the solution reached equilibrium, as determined by HPLC. Similarly, to determine the solubility of BPA in water over a wide temperature range (at neutral $\mathrm{pH}$ ), BPA was added to deionized water until it no longer dissolved and the temperature of the solution was adjusted from $5-45^{\circ} \mathrm{C}$ in the water bath.

In addition, the solubility of BPA in organic solvents (methanol, acetone, dichloromethane, chloroform, ethyl acetate, toluene, or hexane) was determined in the 
same manner by combining solid BPA with the solvent in a centrifuge vial until the BPA no longer dissolved. The temperature was maintained at $25^{\circ} \mathrm{C}$ for all organic solvents. Again, the aliquot of supernatant removed was diluted with methanol and its concentration was determined from methanol standards.

\subsection{Preparation and Characterization of Pristine and Oxidized CNTs}

The specific surface area measurements and transmission electron microscope (TEM) images were provided by Dr. Mohamed Abdel Salam at King Abdulaziz University, Jeddah, Saudi Arabia.

The specific surface area of the pristine CNTs was determined from nitrogen adsorption/ desorption isotherm measurements at $77 \mathrm{~K}$ using a NOVA $3200 \mathrm{e}$ automated gas sorption system (Quantachrome, USA). Each sample was degassed for 6 hours at $100^{\circ} \mathrm{C}$ prior to each measurement.

TEM images of the pristine SWCNTs and L-MWCNTs-10 were obtained.

A $12 \mathrm{M}$ nitric acid solution was prepared by diluting $300 \mathrm{~mL}$ of $16 \mathrm{M}$ nitric acid with 100 $\mathrm{mL}$ of distilled water. To oxidize the carbon nanotubes, $\sim 2 \mathrm{~g}$ of pristine nanotubes (single and multi-walled) were added to $200 \mathrm{~mL}$ of $12 \mathrm{M}$ nitric acid. This mixture was refluxed for twenty-four hours at $140^{\circ} \mathrm{C}$. The CNTs were then filtered using a Millipore filtration apparatus, washed with deionized water until the $\mathrm{pH}$ was neutral and dried in air at $70{ }^{\circ} \mathrm{C}$. 
An ABB Bowen MB Series Arid-Zone spectrometer was used to obtain infrared spectra of the pristine and oxidized CNTs. The $\mathrm{KBr}$ pellet method was used to prepare the CNTs for IR.

Samples of the pristine SWCNTs and L-MWCNTs-10, oxidized SWCNTs and LMWCNsT-10, PP membrane and $0.1 \mu \mathrm{m}$ and $0.65 \mu \mathrm{m}$ PVDF membranes were stuck to aluminum holders using double-sided carbon tape. Each sample was coated with a $24 \%$ $\mathrm{Au} / 76 \% \mathrm{Pd}$ coating by sputtering using a Hummer VII Sputter from Anatech Ltd. (VA, USA). The thickness of the coating was on the order of nanometers. SEM images were obtained using a Tescan Vega II XMU scanning electron microscope (Czech Republic) at a voltage of $20 \mathrm{kV}$.

Aqueous suspensions of pristine and oxidized SWNCTs and L-MWCNTs-10 were prepared by sonicating the CNTs in deionized water for 30 minutes, after which the suspensions were left for several days.

\subsection{CNT Envelope Apparatus}

The stainless steel pieces of the CNT envelope apparatus were designed by the Science Technology Center at Carleton University based on the planned design a CNT envelope. The stainless steel pieces consisted of two punches, a die block, a centering tool, a centering jig, a forming tool, a sealer tool and handle, and a handling tool, as labeled in Figure 2-1. The two punches (top and bottom) were used to cut different sized disks from the PP sheet. The die block acted as a support for the CNT envelope production and had a small indent on the top surface for forming an indent in the PP sheet. The forming 
tool was used to make the indent. The centering jig acted as a cover and fit directly over the die block. The centering tool was used to deliver the CNTs to the membrane and ensure they were collected in the center of the membrane. The sealer tool was used to create the seal between the two pieces of membrane. The handle and handling tool were developed for easier manipulation of the sealer tool. More detailed descriptions of the use of each piece are given in section 2.9, the synthesis of PP-CNT envelopes.

Illustrations, including dimensions, of each piece can be found in Appendix 1. 


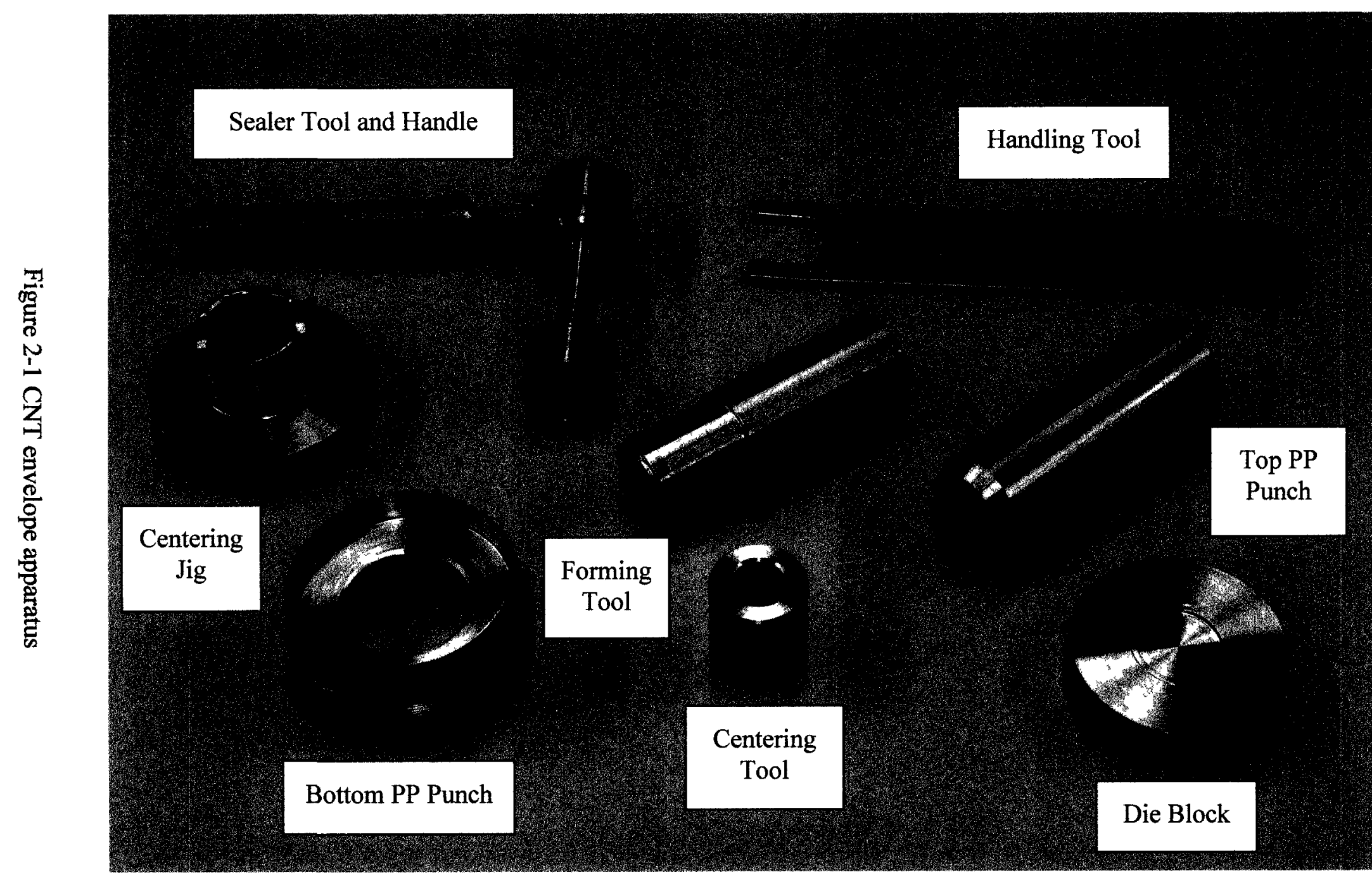




\subsection{Synthesis of PP-CNT Envelopes}

Prior to use, all stainless steel pieces were rinsed with methanol. The propylene membrane used was supplied as a roll, and so, a piece of membrane was cut from the roll and placed on a piece of Plexiglass. The bottom PP punch was used to cut out the large PP disk by pushing the punch into the PP sheet and turning clockwise, shown in Figure 22. A small PP disk was cut using the small PP punch in the same fashion (not shown in images). The large PP disk (diameter: $5 \mathrm{~cm}$ ) and small PP disk (diameter: $2.25 \mathrm{~cm}$ ) are shown in Figure 2-3. The larger of the two cutouts (made from the bottom PP punch) was placed on the die block, with the indent side of the block facing up, and was held in place with the centering jig (Figure 2-4). An indent was imprinted into the PP membrane by pushing the forming tool through the opening in the centering jig onto the PP membrane, shown in Figure 2-5. The large PP membrane is not normally removed from in between the die and centering blocks but has been to illustrate what the indent looks like, as shown in Figure 2-6. Next, the centering tool was guided into the opening of the centering jig and approximately $6.65 \pm 0.05 \mathrm{mg} \mathrm{L}-\mathrm{MWCNTs}-10$ (unless otherwise stated) were transferred to the PP membrane through the hollow core of the centering tool, as shown in Figure 2-7. The centering tool was removed and the small PP cutout (cut from the top PP punch) was placed over the CNTs through the opening of the centering jig, as shown in Figure 2-8. The forming tool was pushed gently on the small PP cutout to ensure it rested flush against the CNTs (not shown). The sealing tool was heated with a heat gun to approximately $210^{\circ} \mathrm{C}^{*}$ and then pushed firmly down on the small PP cutout through the opening in the centering jig, with the aid of the handle and handling tool, 
shown in Figure 2-9 and Figure 2-10, respectively. Figure 2-11 shows the final PP-CNT envelope before and after the extra PP edge has been cut away around the seal.

*The temperature of the sealing tool was measured with a TEGAM $871 \mathrm{~A}$ digitalthermometer as soon as the tool was removed from the heat gun. However, the temperature started to cool as soon as the tool was removed from the heat source, so the reported temperature in the first value measured with the digital thermometer. 


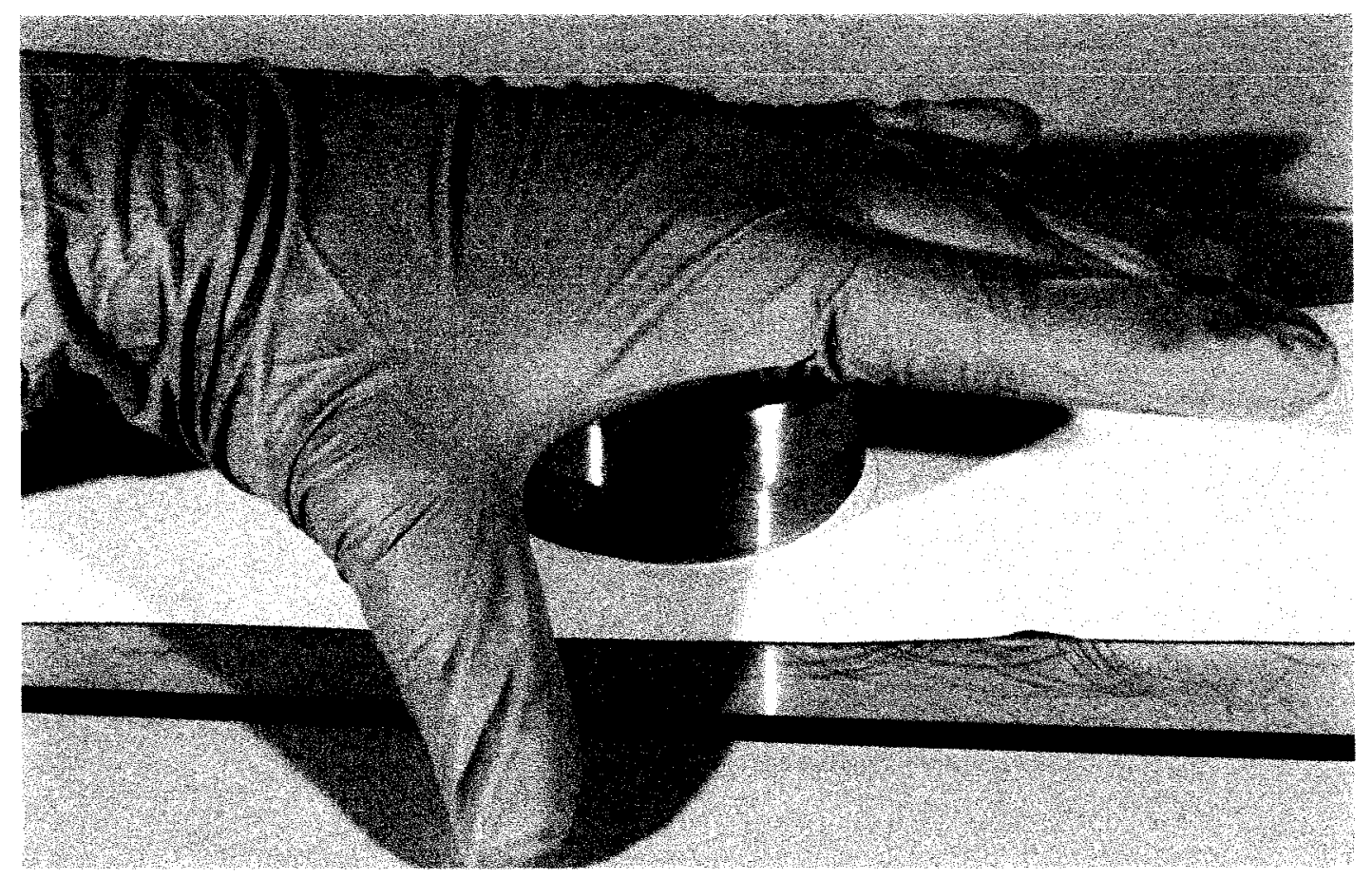

Figure 2-2 The bottom PP punch is used to cut a disk from a sheet of polypropylene by pushing the punch into the membrane and turning clockwise.

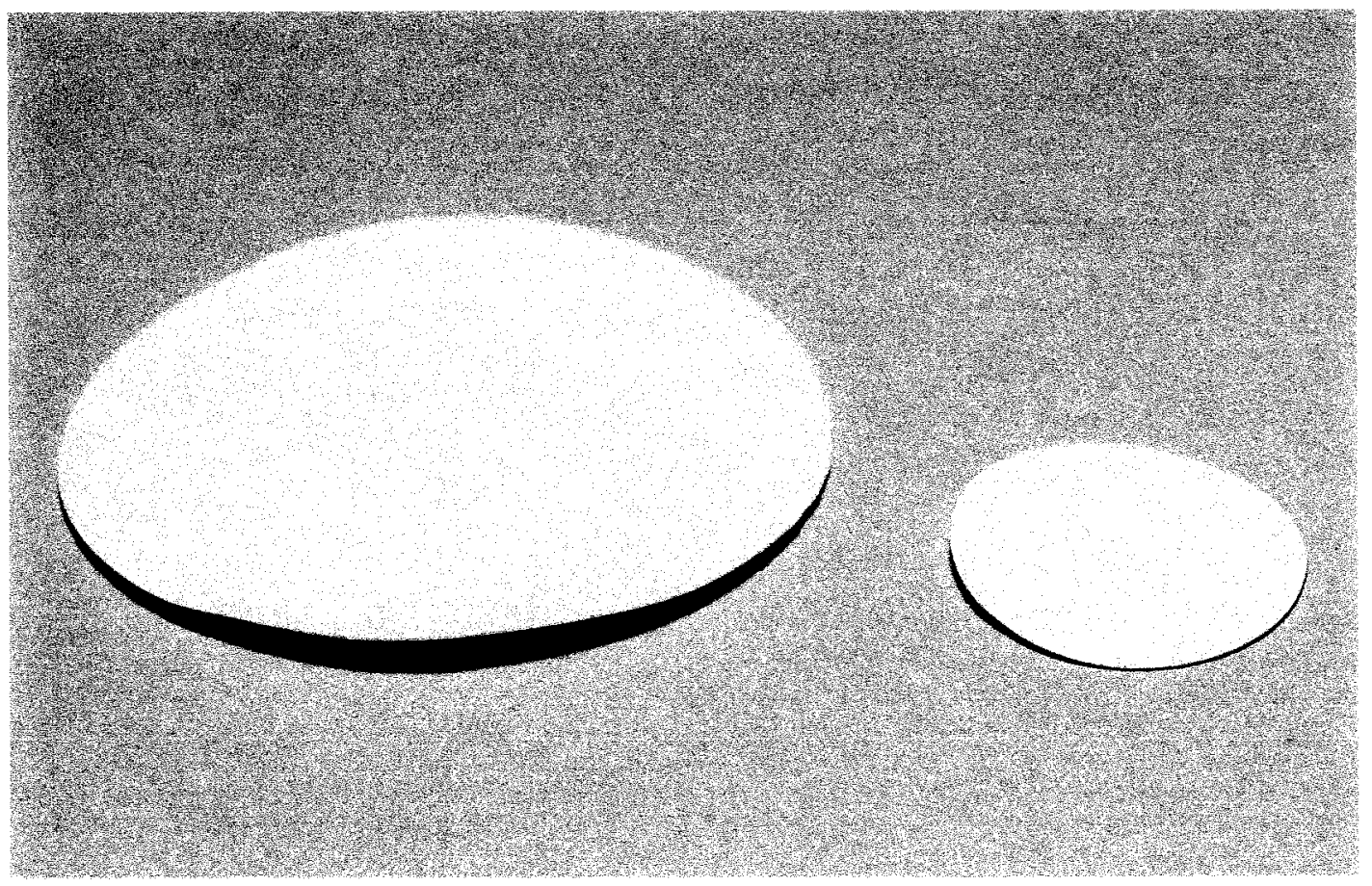

Figure 2-3 The large (diameter: $5 \mathrm{~cm}$ ) and small (diameter: $2.25 \mathrm{~cm}$ ) polypropylene disks cut using the bottom and top PP punches, respectively. 


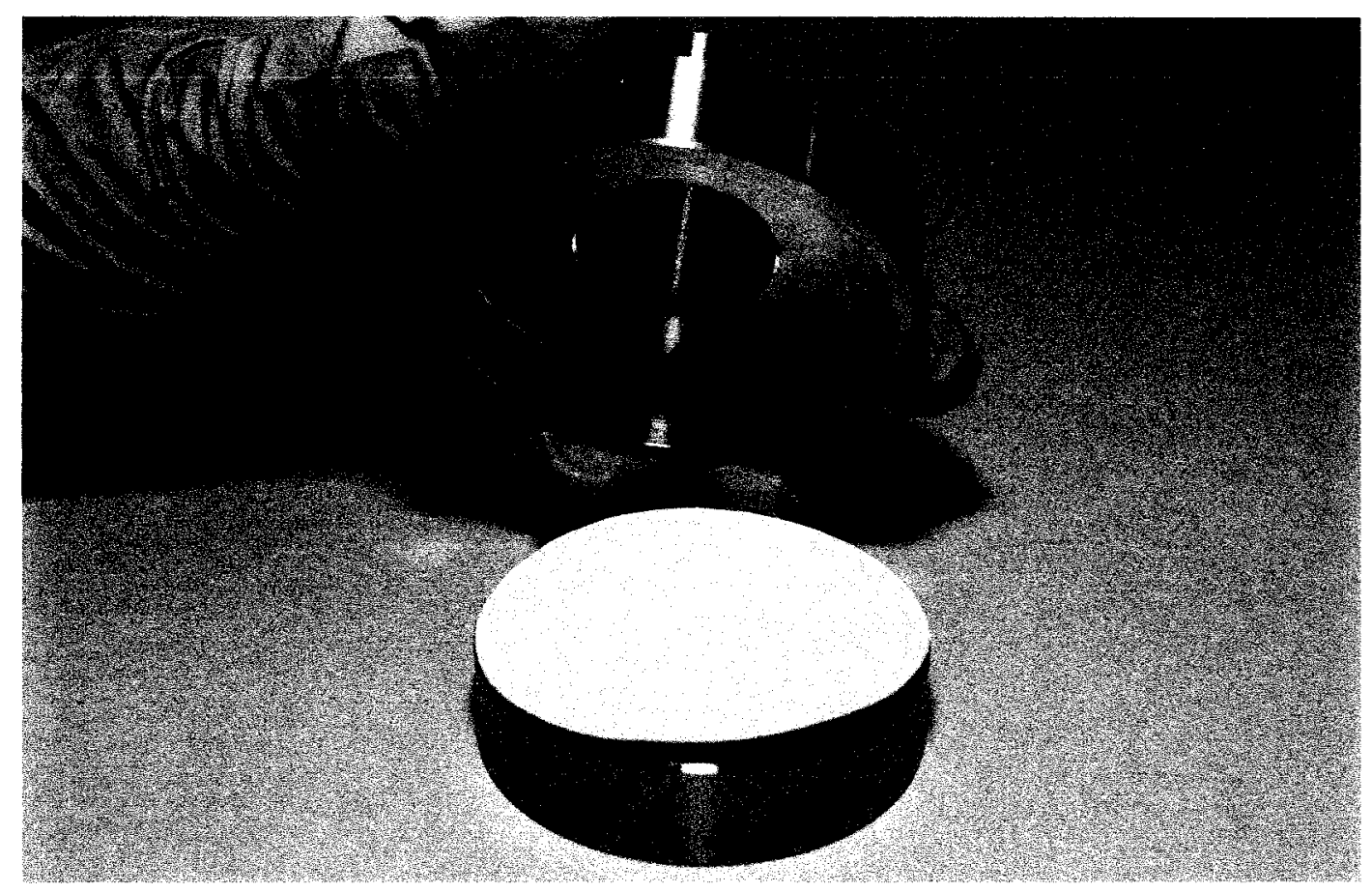

Figure 2-4 The large PP disk is placed on the die block (with indent side facing up) and held in place with the centering jig.

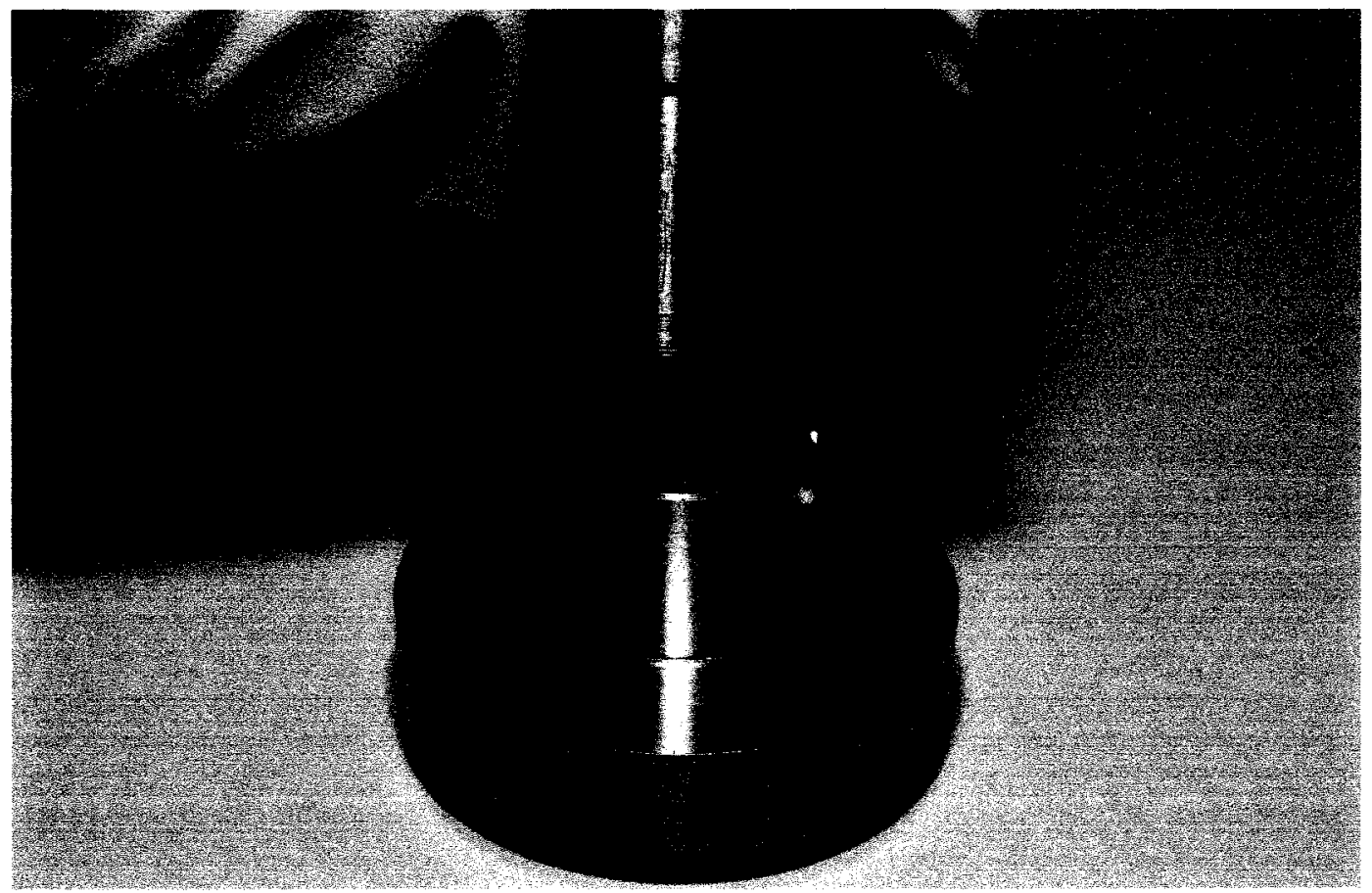

Figure 2-5 The forming tool is guided through the opening in the centering jig and pressed firmly on the large PP disk to create an indent. 


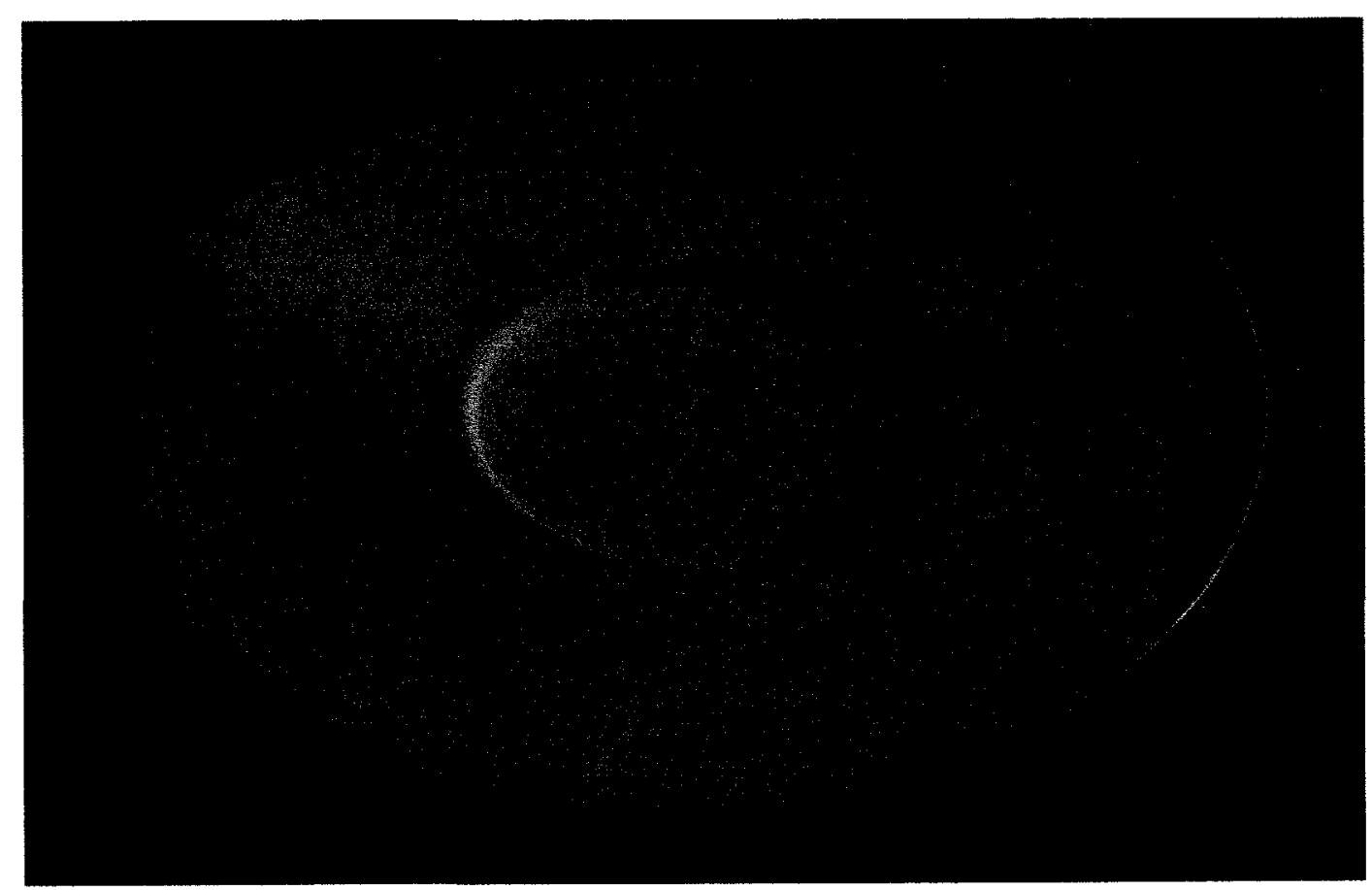

Figure 2-6 Indent in the large PP disk created by the forming tool.

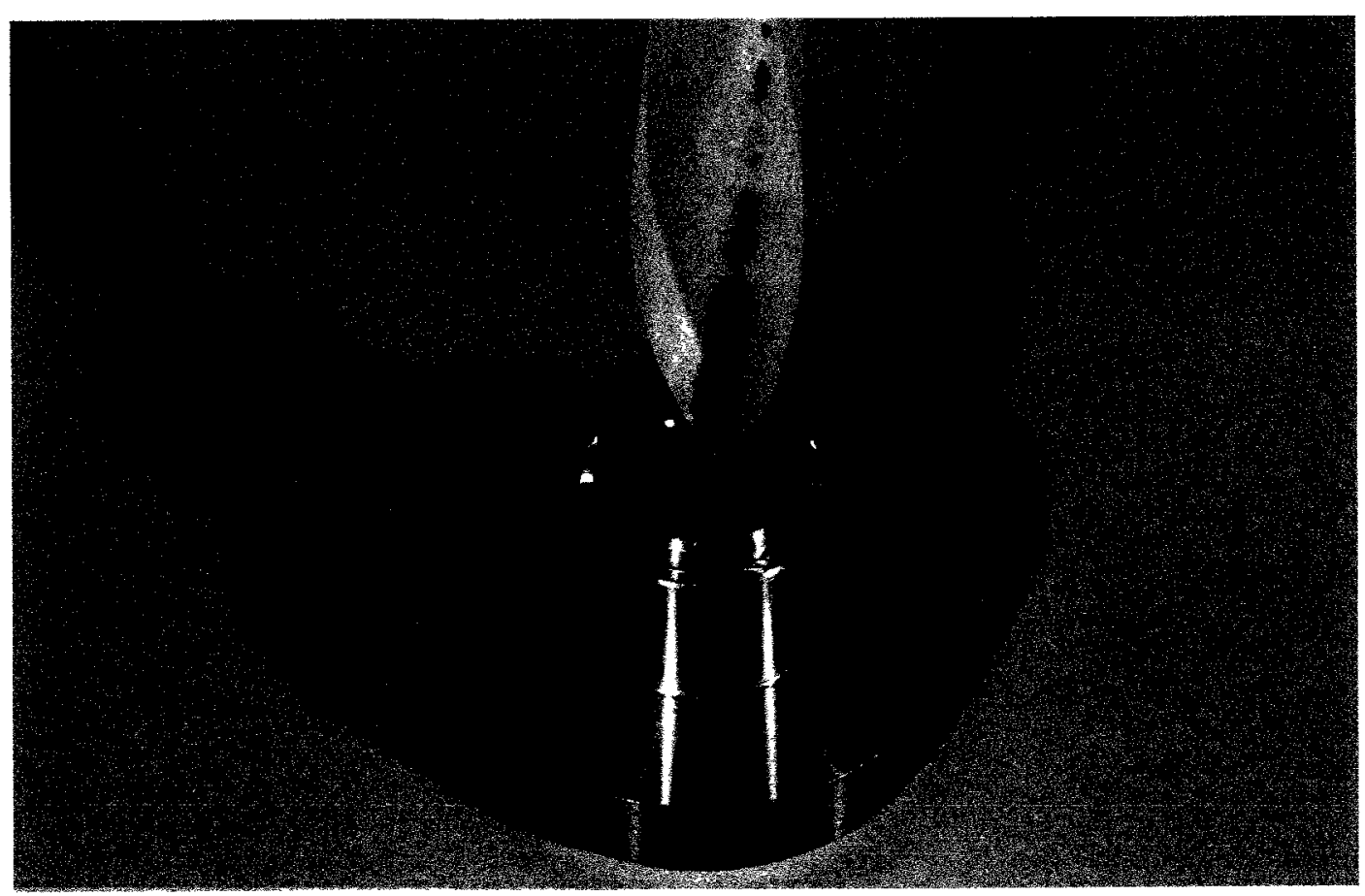

Figure 2-7 The centering tool is inserted into the opening in the centering jig and CNTs are transferred to the surface of the large PP disk through the hollow core of the centering jig. 


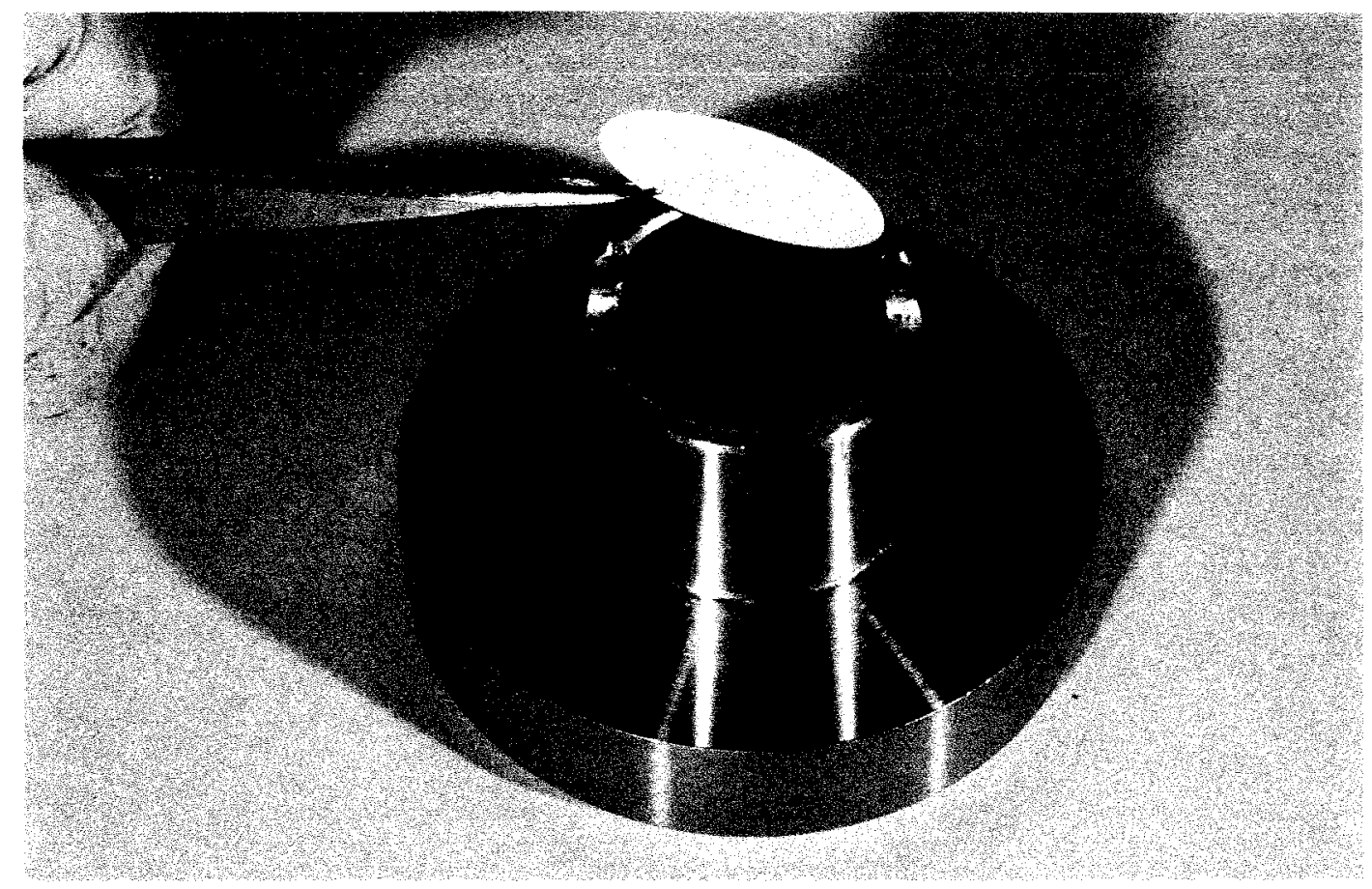

Figure 2-8 The centering tool is removed and the small PP disk is gently placed on top of the CNTs through the opening of the centering jig.

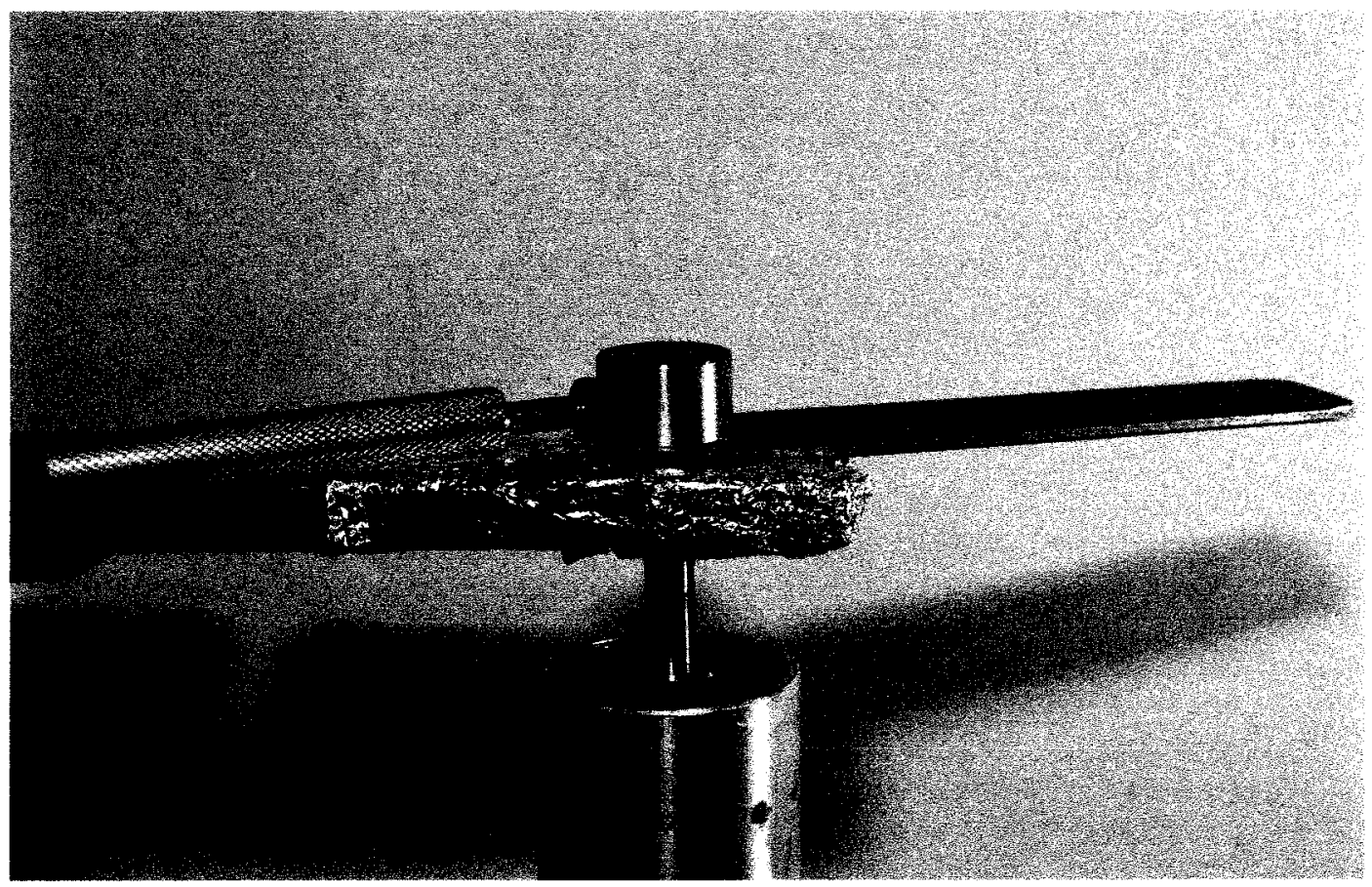

Figure 2-9 The sealer tool is heated to approximately $210^{\circ} \mathrm{C}$ using a heat gun. 


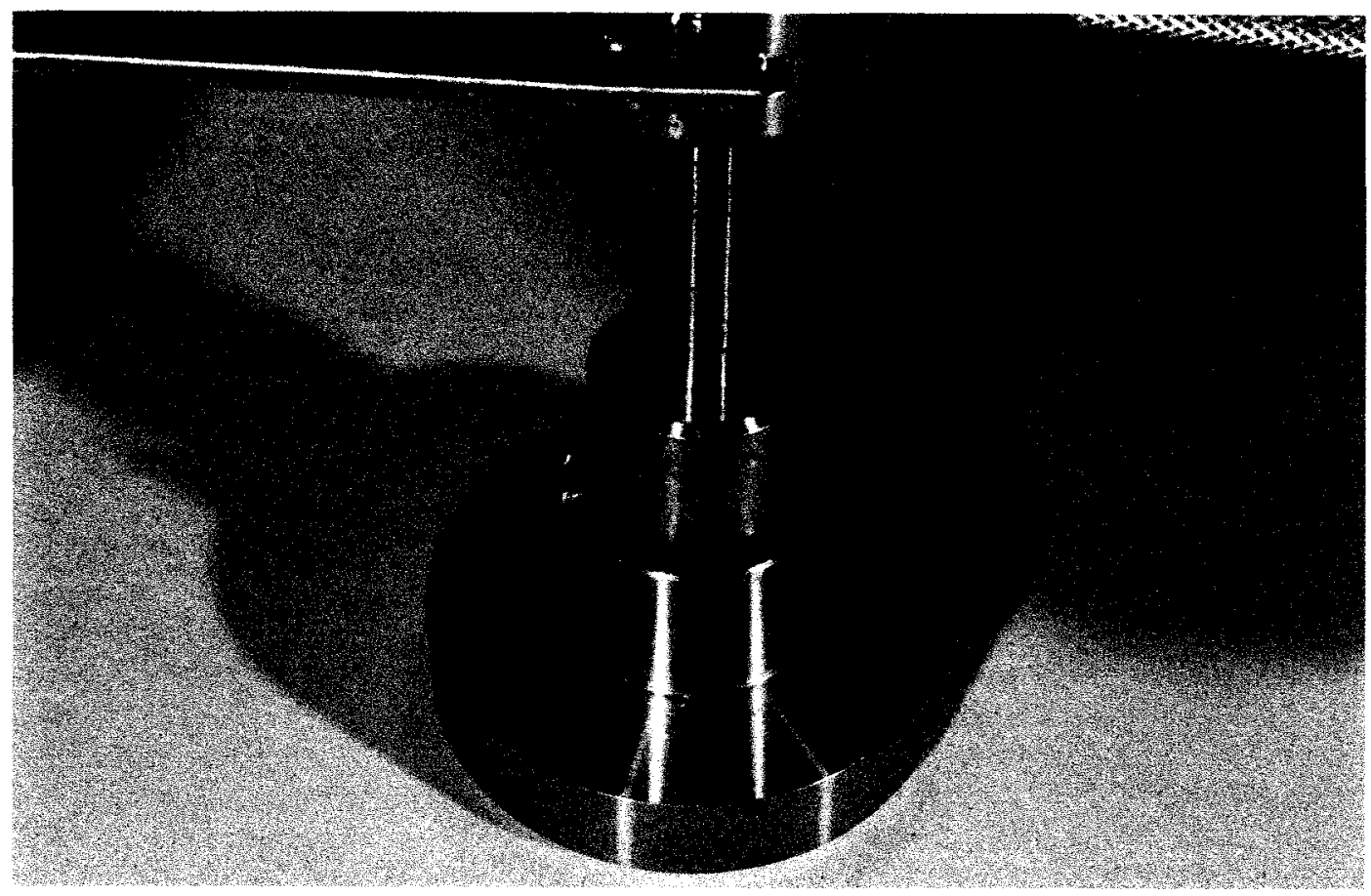

Figure 2-10 The heated sealer tool is guided through the opening of the centering jig and pushed firmly down to create a seal between the small and large PP disks.

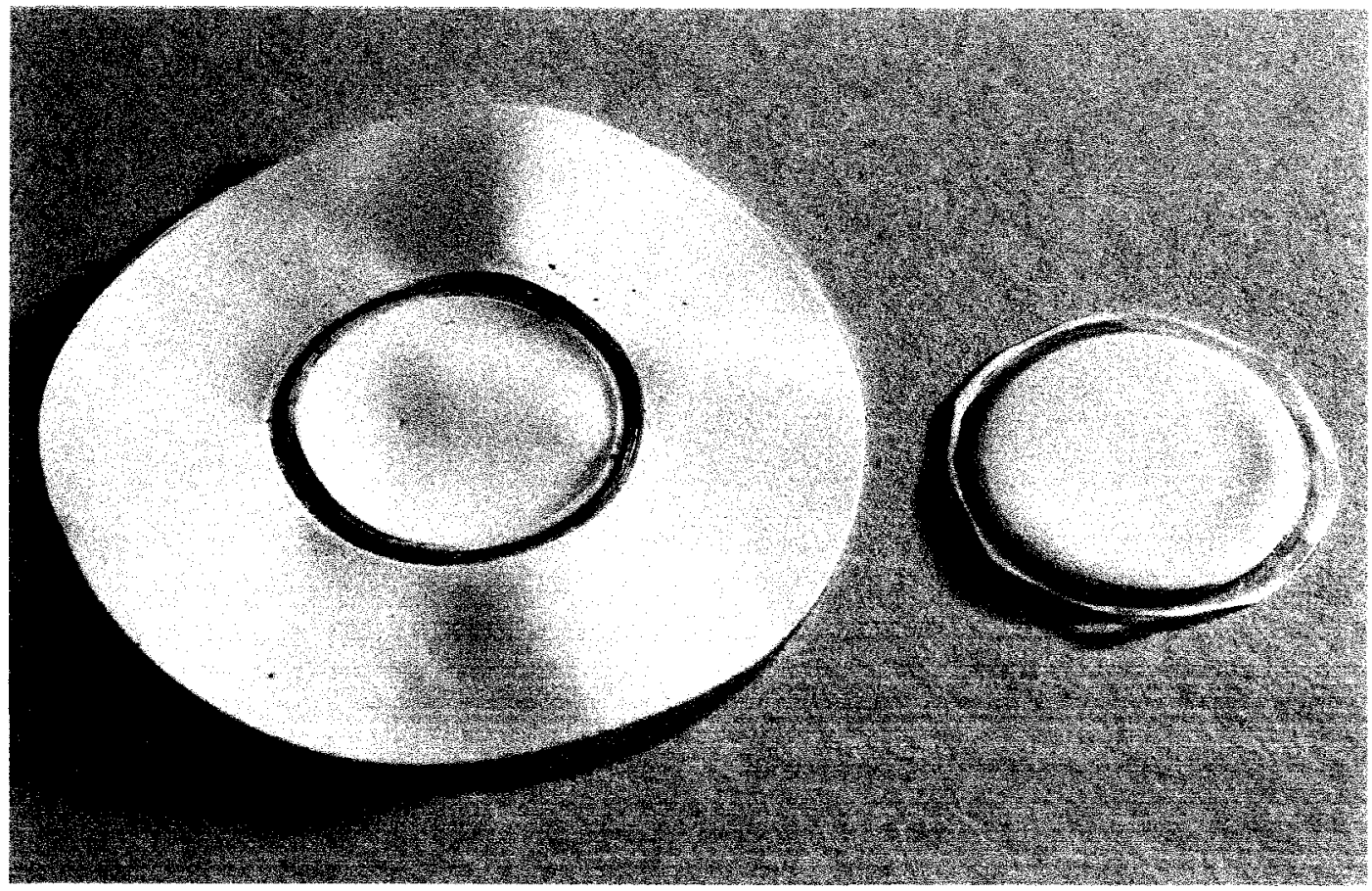

Figure 2-11 A PP-CNT envelope (shown left) and a PP-CNT envelope with excess membrane cut away from the seal (shown right). 


\subsection{Initial BPA Extraction Using PP-CNT Envelopes}

A PP envelope containing pristine long MWCNT with a $10 \mathrm{~nm}$ diameter (L-MWCNT10) was placed in a glass vial containing $40 \mathrm{~mL}$ of $\sim 250 \mathrm{ng} / \mathrm{mL}$ BPA in $\mathrm{dH}_{2} \mathrm{O}$ and was stirred at $240 \mathrm{rpm}$ for 24 hours. The PP-CNT envelope was then removed, blotted dry with a lint-free tissue and transferred to an identical glass vial containing $40 \mathrm{~mL}$ of methanol and was stirred for 24 hours at $240 \mathrm{rpm}$. The amount of BPA left in $\mathrm{dH}_{2} \mathrm{O}$ and the amount of BPA extracted into methanol were determined by HPLC with fluorescence detection. Empty PP envelopes that did not contain any CNTs were also used for extraction under the same experimental conditions.

\subsection{PP-CNT Envelope Conditioning Prior to Adsorption}

Basheer et al. [97] indicated the $\mu$-SPE device was conditioned with organic solvents to improve the extractability of organophosphorous pesticides (OPPs) from sewage sludge samples. The $\mu$-SPE device was immersed in the given organic solvent (methanol, acetone, hexane, dichloromethane, toluene) for approximately one minute, and then ultrasonicated in water for 2 minutes. The authors indicated that only trace amounts of the solvents were retained by the membrane or CNTs in the device. Although the data presented by Basheer et al. [97] about improvements to extractability using conditioning solvents were contradictory, it was decided in the present work to explore the use of conditioning solvents to improve the extraction capacity of the PP-CNT envelopes. In all extractions involving PP-CNT envelope conditioning prior to extraction, both a PP-CNT envelope and a blank PP envelope, without CNTs, were compared and conditioned in the same fashion. 


\subsubsection{Dipping PP-CNT Envelope in an Organic Solvent}

Prior to extraction, the envelope (PP-CNT or empty PP) was dipped in an organic solvent (methanol, acetone, dichloromethane, hexane or toluene) for 1-2 seconds, blotted dry and transferred to a glass vial containing $40 \mathrm{~mL}$ of $\sim 250 \mathrm{ng} / \mathrm{mL} \mathrm{BPA}$ in $\mathrm{dH}_{2} \mathrm{O}$. The rest of the extraction proceeded under the same conditions as the initial BPA extraction using a PP-CNT envelope.

\subsubsection{Soaking PP-CNT Envelope in an Organic Solvent}

Prior to extraction, the envelope (PP-CNT or empty PP) was soaked in an organic solvent (acetone or methanol) overnight and blotted dry and transferred to the glass vial containing BPA in $\mathrm{dH}_{2} \mathrm{O}$ as above.

\subsubsection{Dipping the Rolled PP-CNT Envelope in an Organic Solvent}

The envelope (PP-CNT or empty CNT) was rolled tightly on itself and held in place using a pair of tweezers. The curled envelope was dipped in either acetone or methanol, blotted dry and transferred to $\mathrm{BPA}$ in $\mathrm{dH}_{2} \mathrm{O}$ as above.

\subsubsection{Sonicating the PP-CNT Envelope in an Organic Solvent}

The envelope (PP-CNT or CNT) was sonicated in either acetone or methanol for 10 minutes, blotted dry and then transferred to $\mathrm{BPA}$ in $\mathrm{dH}_{2} \mathrm{O}$ as above.

\subsubsection{Change in Mass of Conditioned PP-CNT Envelope over Time}

A PP-CNT envelope was dipped in acetone for a timed period (1-60 seconds), blotted dry and immediately weighed to determine what volume of acetone filled the envelope in 
the given time frame. This process was repeated several times with the same envelope, varying the "dipping" time in each case. The whole experiment was repeated with methanol and hexane. In addition, two polypropylene disks (cut using the small punch) were used to repeat the same procedure in all three solvents, to determine how much solvent was absorbed by the membrane.

\subsection{Synthesis of PVDF-CNT Envelopes}

A Millipore fritted glass filter was fitted into a $500 \mathrm{~mL}$ filter flask and connected to vacuum. A PVDF membrane was placed on the fritted glass filter holder, and then the centering tool was placed approximately in the center of the membrane. Approximately $6.65 \pm 0.05 \mathrm{mg}$ CNTs were transferred to the surface of the PVDF membrane by pouring the CNTs through the hollow core of the centering tube, as shown in Figure 2-12. Next, 1-2 mL of methanol was added to the CNTs through the hollow core of the centering tool with a Pasteur pipette (Figure 2-13). Once the methanol filtered through the filter support the centering tool was carefully removed and the PVDF membrane, with the CNTs collected in the centre, was placed on the underside (without the indent) of the die block, as illustrated in Figure 2-14. A second PVDF membrane was placed on top of the first and held in place with the centering jig. The sealer tool was heated and used to create a stamp as described in the synthesis of PP-CNT envelopes. Figure 2-15 shows the PVDFCNT envelopes before and after the excess edge has been cut away.

During extraction, the PVDF-CNT envelopes were suspended in the vial using either a twist-lock cable fastener or a piece of Teflon tubing, as shown in Figure 2-16. To hold the envelopes in place, the fastener was twisted around the edge of the PVDF-CNT 
envelope. Using the Teflon tube, a small slit was cut halfway through the tube horizontally and the envelope was fitted into the slit. Both the twist-lock and the Teflon tube were suspended using Teflon-coated electrical wire. 


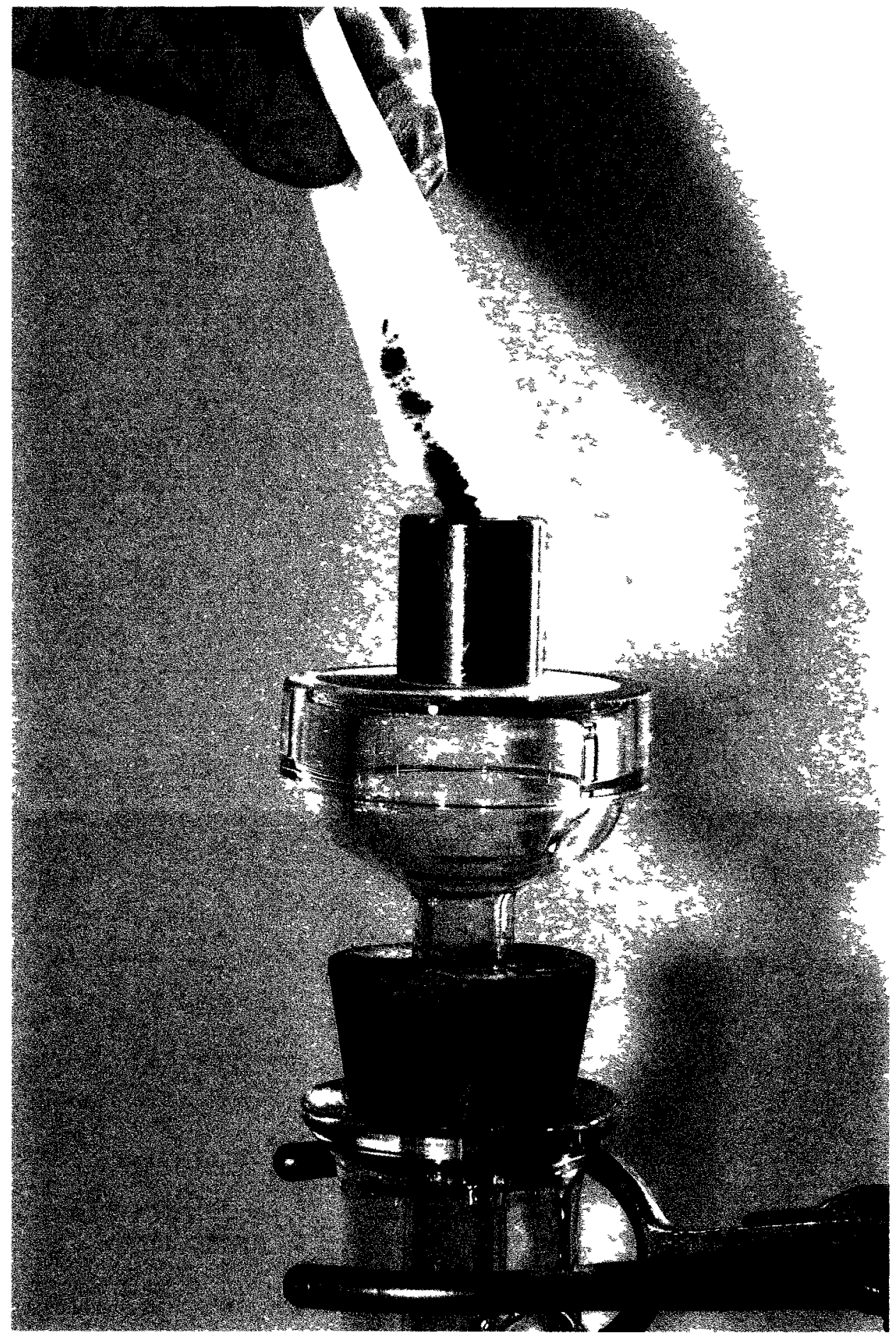

Figure 2-12 A polyvinylidene fluoride disk is placed on a Millipore filter support and the centering tool is placed on top so that the opening of the tool is approximately in the center of the membrane. CNTs are introduced to the membrane through the hollow core of the centering tool. 


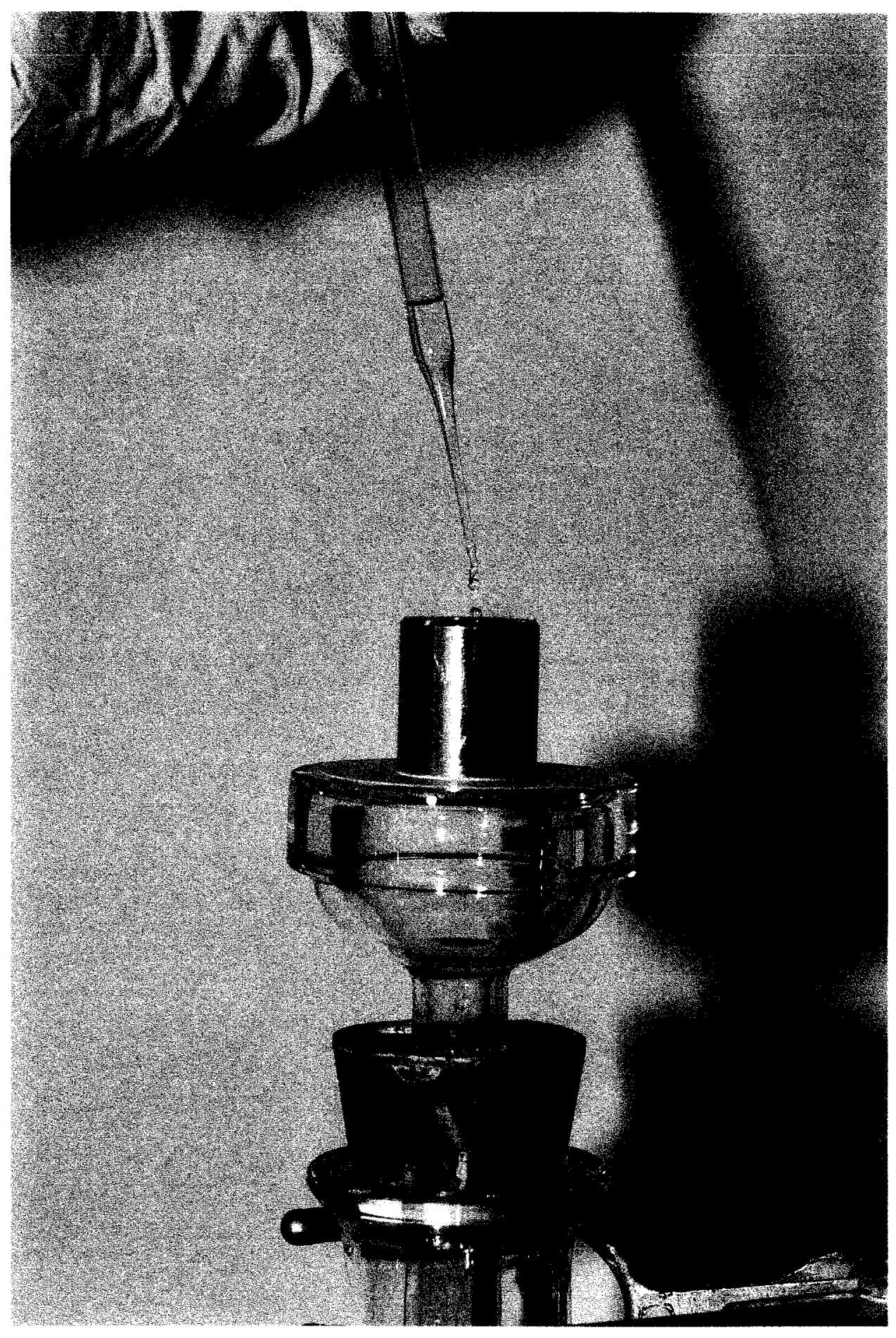

Figure 2-13 Methanol is introduced through the hollow core of the centering tool to wet the CNTs. 


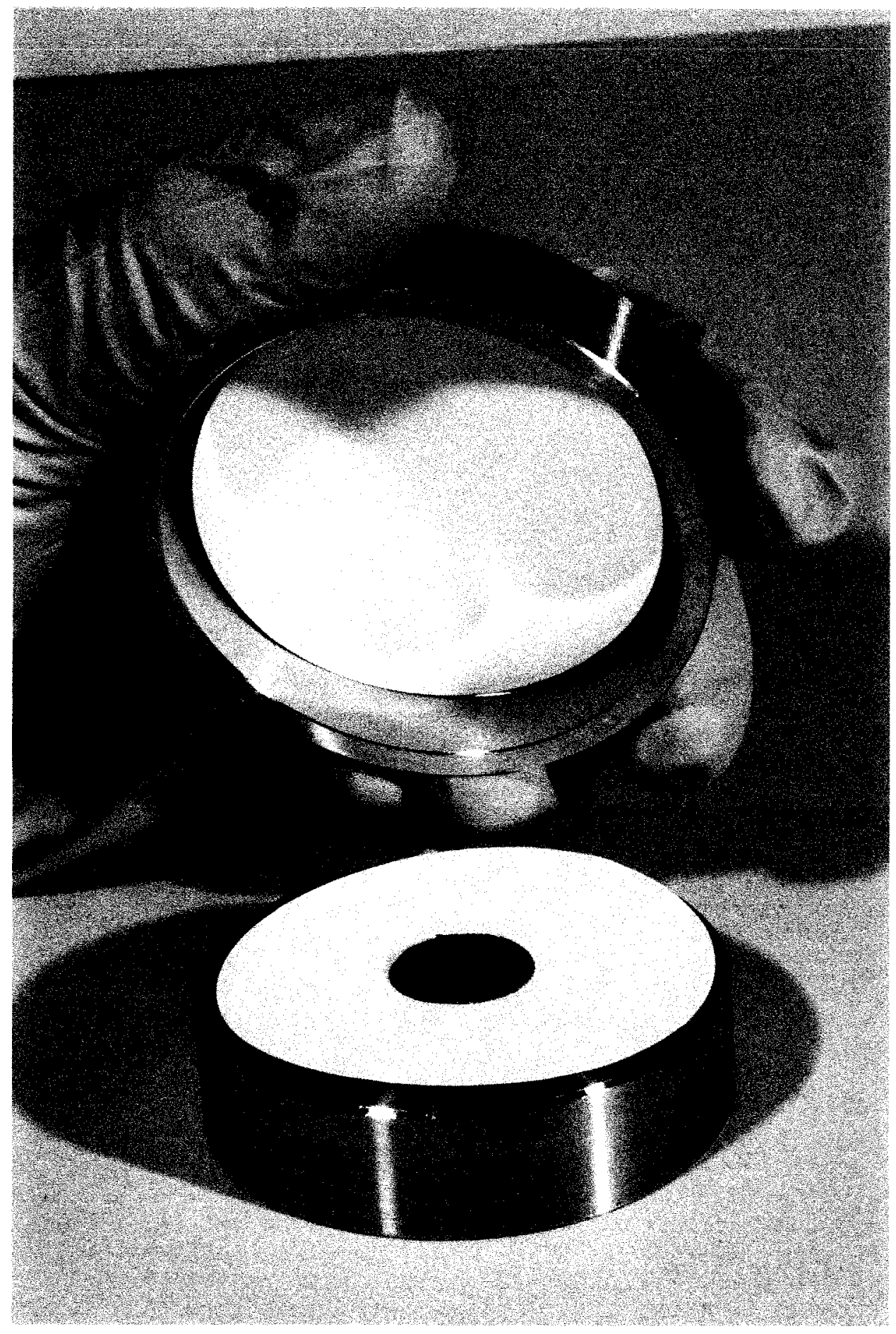

Figure 2-14 The PVDF disk with CNTs collected in the center, is placed on the die block with indent side down. A second PVDF disk is placed over top of the disk containing the CNTs, guided by the centering jig. 


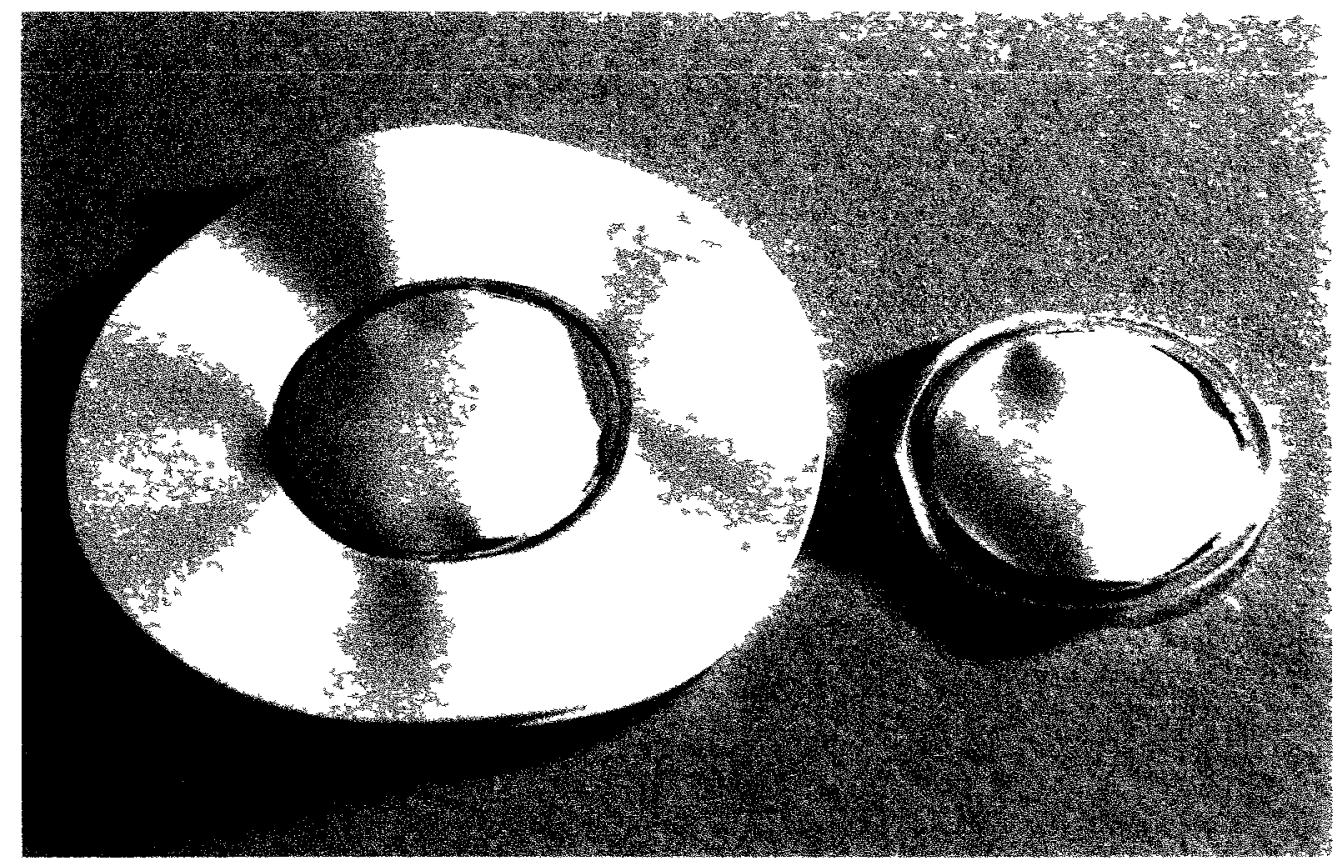

Figure 2-15 A PVDF-CNT envelope (shown left) and a PVDF-CNT envelope with excess membrane cut away from the seal (shown right).

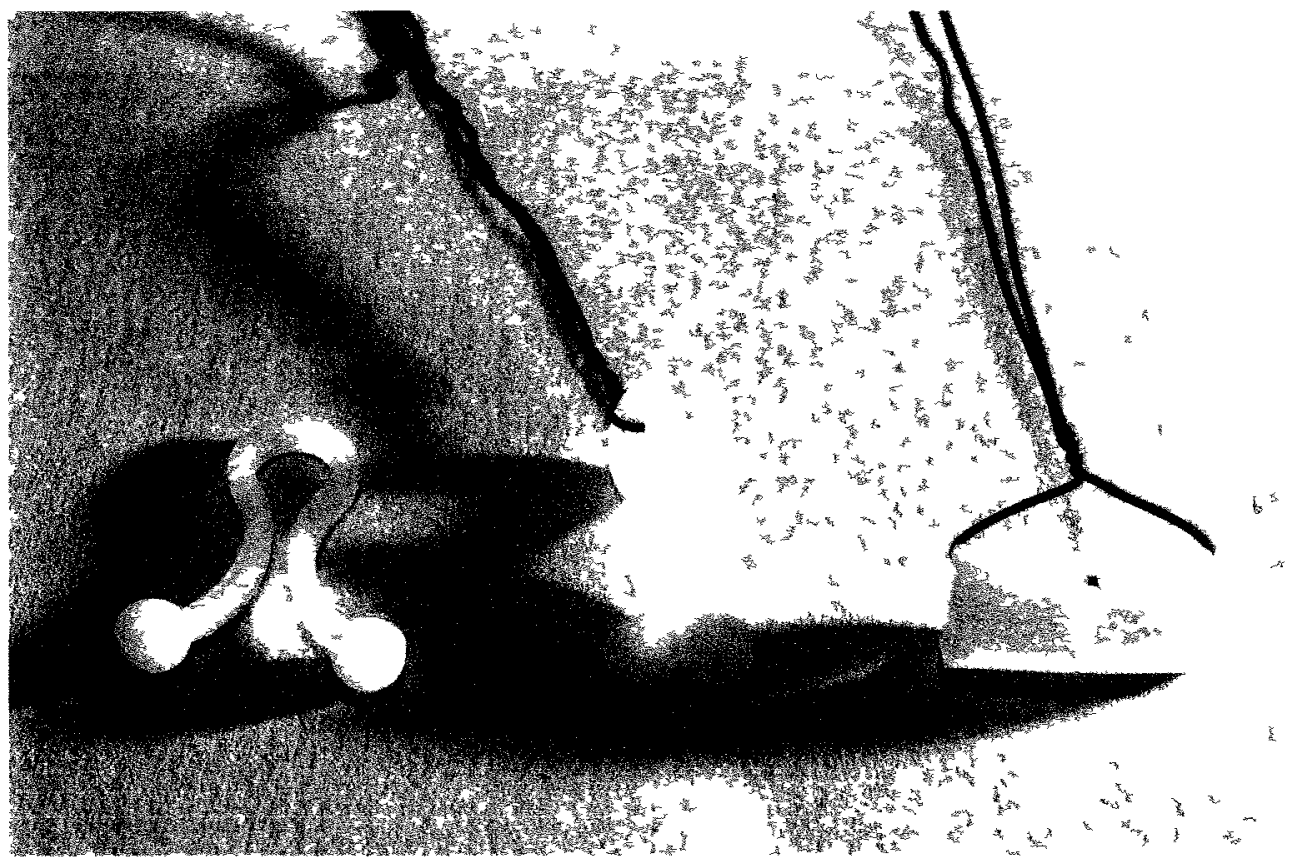

Figure 2-16 Open and closed twist-lock cable fasteners (shown left and center, respectively) and a piece of Teflon tubing with a slit cut half-way through the tube (shown right). Teflon-coated wire was threaded through the opening at the top of the closed twist-lock and through the core of the Teflon tube to suspend the CNT envelopes. 


\subsection{Initial Extraction of BPA Using PVDF-CNT Envelope}

A PVDF-CNT envelope was clamped between the two ends of a twist-lock cable fastener and suspended in a glass vial containing $40 \mathrm{~mL}$ of $250 \mathrm{ng} / \mathrm{mL} \mathrm{BPA}$ in $\mathrm{dH}_{2} \mathrm{O}$ using a piece of Teflon-coated electrical wire. The solution was stirred at $\sim 240 \mathrm{rpm}$ for 24 hours. Next, the suspended PVDF-CNT envelope was removed from $\mathrm{dH}_{2} \mathrm{O}$, blotted dry with a lint-free tissue and transferred to an identical glass vial containing $40 \mathrm{~mL}$ of methanol. Again, the solution was stirred at $\sim 240 \mathrm{rpm}$ for 24 hours. This procedure was repeated with four more PVDF-CNT envelopes. In addition, five empty PVDF envelopes were extracted under the same conditions.

Furthermore, to ensure the polymeric coating on the stirrer bars did not act as a sorbent, five stirrer bars were extracted under the same conditions, without any envelopes present. To confirm the twist-lock fasteners also did not participate in BPA extraction, extractions using only the twist-lock were also performed with stirring under the same conditions.

After determining the twist-lock also acted as a sorbent, extraction was repeated wherein the twist-lock was used to suspend the PVDF-CNT envelopes, but the envelope was desorbed in methanol separate from the twist-lock.

To determine the sorptive properties of the Teflon tubing extraction was performed by suspending the Teflon tubing in aqueous BPA solution with stirring using the same adsorption and desorption conditions. 
BPA carryover from one envelope to the next was determined by performing an extraction as described but desorbing the envelopes in a second $15 \mathrm{~mL}$ aliquot of methanol.

The reusability of a single PVDF-CNT envelope was performed by extracting and desorbing BPA from the envelope as previously described. Several hours to a day was waited between extractions to ensure any residual methanol had evaporated from the envelope.

\subsection{BPA Adsorption to PVDF-CNT Envelopes}

Please note: in every adsorption experiment the envelopes were suspended in $40 \mathrm{~mL}$ of $\sim 250 \mathrm{ng} / \mathrm{mL}$ BPA in $\mathrm{dH}_{2} \mathrm{O}$ for 24 hours with stirring at $\sim 240 \mathrm{rpm}$ and transferred to $15 \mathrm{~mL}$ of methanol for 1 hour with stirring for desorption, unless stated otherwise.

\subsubsection{BPA Adsorption Rate}

The rate of BPA adsorption to CNT envelopes was explored three times, with and without stirring using envelopes made from $0.1 \mu \mathrm{m}$ pore diameter membrane, and with stirring using envelopes made from the $0.65 \mu \mathrm{m}$ pore diameter PVDF membrane. In each experiment, several PVDF-CNT envelopes were suspended in aqueous BPA solution for a different length of time. The adsorption rate was explored from 1-41 hours for the 0.1 $\mu \mathrm{m}$ PVDF-CNT envelopes with stirring, 1-90 hours for the same envelopes without stirring, and 1-32 hours with the $0.65 \mu \mathrm{m}$ PVDF-CNT envelopes. 
Also, a single PVDF-CNT envelope was sonicated in $20 \mathrm{~mL}$ aqueous BPA solution for 30 minutes and desorbed in methanol as described above. For reasons discussed in Section 3.9.1.2 the adsorption rate was not measure using sonication.

\subsubsection{Effect of Temperature}

PVDF-CNT envelopes were suspended in aqueous BPA solution and the vials were placed in a Neslab RTE-8 refrigerated circulating water bath, where the temperature was controlled from $5-45^{\circ} \mathrm{C}$. Since magnetic stirring was not used, the PVDF-CNT envelopes were suspended for 72 hours to ensure adsorption reached equilibrium. Desorption was performed with the conditions stated above, at room temperature.

\subsubsection{Effect of Concentration of Aqueous BPA solution}

Several PVDF-CNT envelopes were suspended in $40 \mathrm{~mL}$ of aqueous BPA solutions with varying concentrations, $0.5-250 \mathrm{ng} / \mathrm{mL}$. The PVDF-CNT envelopes were transferred to $1 \mathrm{~mL}$ of methanol for 2 hours, without stirring.

\subsubsection{Effect of Sample Volume}

The effect of sample volume on BPA extraction using CNT envelopes was explored twice. First, aqueous BPA solutions were prepared in volumes ranging from 15-2000 mL with constant BPA concentration of $\sim 250 \mathrm{ng} / \mathrm{mL}$. Second, the aqueous BPA solutions were prepared in the same volumes, but with a constant mass of $10 \mathrm{ng}$ of BPA in each solution. $C_{1} V_{1}=C_{2} V_{2}$ calculations were used to determine what volume of a $1000 \mathrm{ng} / \mathrm{mL}$ BPA in $\mathrm{dH}_{2} \mathrm{O}$ standard needed to be diluted to prepare the solutions with the appropriate 
concentration or mass of BPA, respectively. The PVDF-CNT envelopes were transferred to $1 \mathrm{~mL}$ of methanol for 2 hours, without stirring.

\subsubsection{Effect of Mass of CNTs}

Several PVDF-CNT envelopes were prepared with CNT mass ranging from 0.5-13.3 mg, as described in Section 2.11.5. Adsorption and desorption were performed as stated above.

\subsubsection{Effect of Type of CNTs}

Several PVDF-CNT envelopes were prepared with $\sim 6.65 \mathrm{mg}$ of pristine SWCNTs, DWCNTs, L-MWCNTs-10, L-MWCNTs-1020, S-MWCNTs-1020, oxidized SWNCTs or oxidized MWCNTs as described in Section 2.4. Adsorption and desorption were performed as stated above.

\subsubsection{Effect of pH on BPA Extraction}

Several $\sim 250 \mathrm{ng} / \mathrm{mL}$ BPA in $\mathrm{dH}_{2} \mathrm{O}$ standards were prepared from a $1000 \mathrm{ng} / \mathrm{L} \mathrm{BPA}$ in $\mathrm{dH}_{2} \mathrm{O}$ stock solution. The $\mathrm{pH}$ of each solution was varied by adding a small amount of dilute $\mathrm{HCl}$ or $\mathrm{NaOH}$ to each flask prior to dilution with water. $\mathrm{pH}$ values were measured using a Fisher Accumet Model 210 pH meter.

\subsection{BPA Desorption to PVDF-CNT Envelopes}

Please note: in every desorption experiment the envelopes were suspended in $40 \mathrm{~mL}$ of $\sim 250 \mathrm{ng} / \mathrm{mL}$ BPA in $\mathrm{dH}_{2} \mathrm{O}$ for 24 hours with stirring at $\sim 240 \mathrm{rpm}$. 


\subsubsection{BPA Desorption Rate}

The desorption rate was also explored with and without stirring. In either experiment, the PVDF-CNT envelopes were suspended in $40 \mathrm{~mL}$ of methanol for different amounts of time with or without stirring at $\sim 240 \mathrm{rpm}$. The desorption rate was explored from 1-180 minutes both experiments.

\subsubsection{Desorption Solvent Volume}

The volume of methanol needed for desorption was also studied with and without stirring. When stirring was used, several PVDF-CNT envelopes were suspended in 15-40 $\mathrm{mL}$ of methanol for one hour. Without stirring, the envelopes were immersed in 1-10 mL methanol for two hours.

\subsubsection{Effect of Desorption Solvent}

BPA recovery was explored when the PVDF-CNT envelopes were desorbed in $15 \mathrm{~mL}$ of methanol, acetone or acetonitrile with stirring. The concentration of BPA extracted in each solvent was determined from a calibration curve of BPA standards prepared in the corresponding solvent.

\subsection{BPA Extraction from Plastic Bottle}

Several polycarbonate baby bottles were obtained from local and international sources. Several different techniques were used to attempt to leach BPA from the bottle in deionized water. A photograph of all of the bottles used is shown in Figure 2-17. 


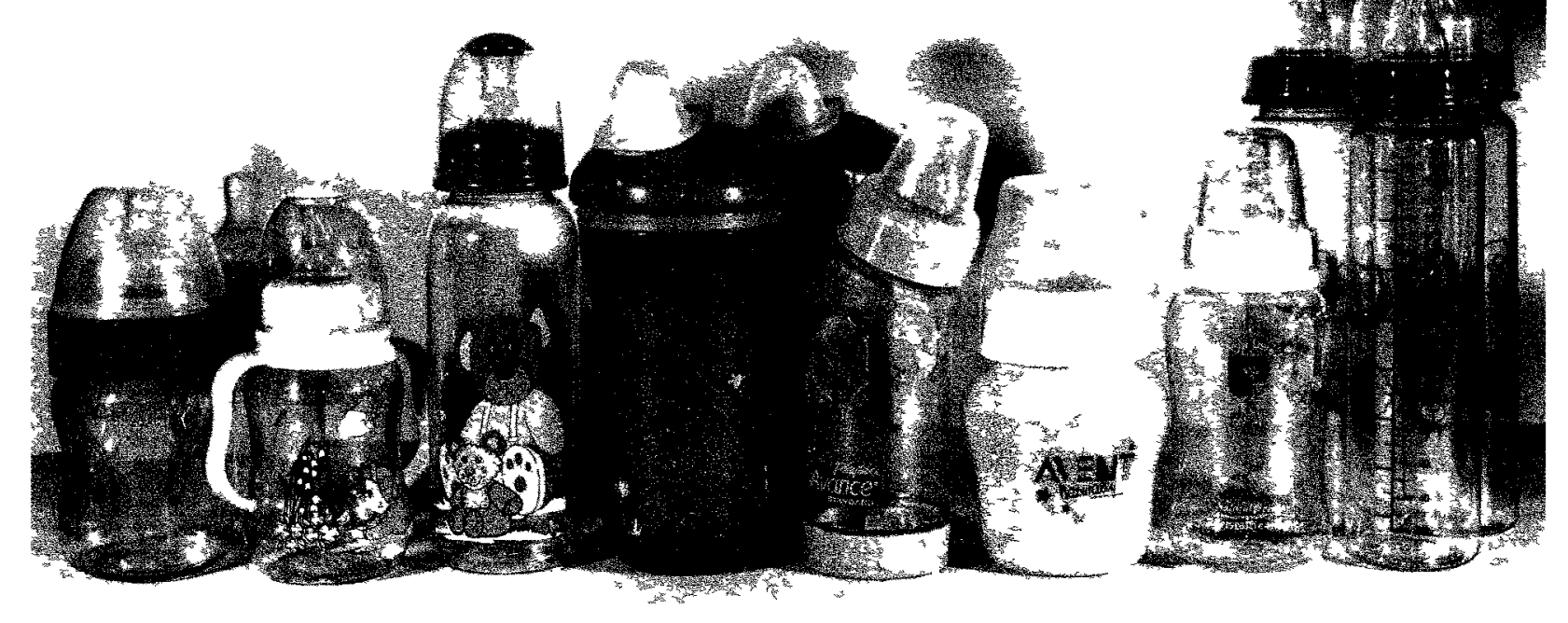

Figure 2-17 The baby bottles used for practical application of BPA extraction from aqueous solution using PVDF-CNT envelopes. From left: A1, A2 (behind A1), B1, C1, D1 (orange), D2 (purple, behind D1), E1, F1, G1, H1, H2 and H3 (both behind H1).

For BPA leaching using bottles $\mathrm{A} 1, \mathrm{~A} 2$ and $\mathrm{B} 1$, deionized water was heated to $85^{\circ} \mathrm{C}$ and then $170 \mathrm{~mL}, 160 \mathrm{~mL}$, and $150 \mathrm{~mL}$ was transferred to $\mathrm{A} 1, \mathrm{~A} 2$ and $\mathrm{B} 1$, respectively. The bottles were maintained at $85^{\circ} \mathrm{C}$ for 30 minutes in an oven. After cooling to room temperature, extractions were performed by suspending PVDF-CNT envelopes in $15 \mathrm{~mL}$ of $\mathrm{dH}_{2} \mathrm{O}$ from each bottle for 24 hours with stirring. During adsorption, the rest of the water in the bottle was transferred to a volumetric flask to prevent further leaching. To elute BPA from the envelope, it was immersed in $1 \mathrm{~mL}$ methanol for 2 hours, without stirring. Repeat experiments were performed with the same water in the same bottles. The water originally used for extraction was heated again, and transferred back to the 
bottle to induce further leaching from the plastic. The same procedure was repeated for successive BPA extractions, except the $\mathrm{pH}$ of the water was made basic by dissolving a $\mathrm{NaOH}$ pellet in the water prior to heating and then acidic with $\mathrm{HCl}$ prior to adsorption.

Please note: For every bottle described hereafter, for adsorption, a PVDF-CNT envelope was suspended in $15 \mathrm{~mL} \mathrm{dH_{2 }} \mathrm{O}$ from the bottle, after leaching for 24 hours with stirring. The $\mathrm{pH}$ of the water was made acidic with $\mathrm{HCl}$. Desorption was performed by immersing the envelope in $1 \mathrm{~mL}$ methanol for 2 hours.

For BPA leaching using bottle $\mathrm{C} 1$, basic water $(\mathrm{pH} 11-12)$ was heated to $85^{\circ} \mathrm{C}$ and then $270 \mathrm{~mL}$ was transferred to $\mathrm{C} 1$, and the bottle was maintained at $85^{\circ} \mathrm{C}$ for 24 hours in an oven. The bottle was left to cool overnight on the lab bench. Adsorption and desorption were performed as stated. Only one experiment was performed with $\mathrm{C} 1$.

Bottles D1, D2 and E1 were filled with $275 \mathrm{~mL}, 240 \mathrm{~mL}$ and $235 \mathrm{~mL}$ basic deionized water, respectively and left on the lab bench for 24 hours. Adsorption and desorption were performed as stated. Repeat experiments were performed with the previously used water in the same bottles.

Bottles $\mathrm{F} 1$ and $\mathrm{G} 1$ were filled with $200 \mathrm{~mL}$ and $215 \mathrm{~mL}$ of basic deionized $\mathrm{H}_{2} \mathrm{O}$, respectively and heated in a microwave for 45 seconds. The bottles were left to cool to room temperature prior to extraction. Adsorption and desorption were performed as stated. Repeat experiments were performed with the previously used water in the same bottles. 
Basic water was heated to $50^{\circ} \mathrm{C}$ and then $230 \mathrm{~mL}$ was transferred to each of $\mathrm{H} 1, \mathrm{H} 2$ and H3. The bottles were left to cool to room temperature prior to extraction. Adsorption and desorption were performed as stated.

\subsection{HPLC with Fluorescence Detection}

HPLC detection was performed using a Varian 9010 Solvent Delivery System with a Linear Fluor LC 304 fluorescence detector and a Varian 9050 Autosampler. A Genesis C18 column (length: $120 \mathrm{~mm}$, internal diameter: $4 \mu \mathrm{m}$ ) was used. A gradient program was used for the mobile phase with an initial composition of $90 \%$ methanol: $10 \%$ water which changed to $100 \%$ methanol in 2 minutes, and was then held for two minutes. The flow rate of the mobile phase was $1.0 \mathrm{~mL} / \mathrm{min}$. The optimum excitation and emission wavelengths, $224 \mathrm{~nm}$ and $310 \mathrm{~nm}$ respectively, were determined using a Varian Cary Eclipse Fluorescence Spectrophotometer.

For the analysis of the methanol extracts from the extractions using polycarbonate baby bottles, the HPLC method was changed to: an initial composition of 50\% methanol: $50 \%$ water which changed to $100 \%$ methanol in 10 minutes, then held for 4 minutes. The flow rate was not changed. 


\section{DISCUSSION}

\subsection{Analytical Method}

Originally, separate stock solutions of $100 \mu \mathrm{g} / \mathrm{mL}$ BPA in methanol and water were prepared. When the $100 \mu \mathrm{g} / \mathrm{mL}$ BPA in water stock was diluted to prepare a $250 \mathrm{ng} / \mathrm{mL}$ BPA in water standard, its concentration was determined to be significantly lower (up to $\sim 100 \mathrm{ng} / \mathrm{mL}$ less) than $250 \mathrm{ng} / \mathrm{mL}$ when it was calculated from a calibration curve of BPA in methanol standards. The actual concentration of the BPA in the water stock solution was likely significantly lower than the believed concentration due to one of two reasons. The reported solubility of BPA in water ranges from $120-330 \mu \mathrm{g} / \mathrm{mL}$ and this value will easily fluctuate with temperature variations. Therefore, it is quite possible that not all of the BPA in the stock solution dissolved since $100 \mu \mathrm{g} / \mathrm{mL}$ is so close to the lower end of the maximum solubility range. Furthermore, the actual concentration of the stock was determined using methanol standards even though it was an aqueous stock solution. It was later determined that two BPA standards in methanol and water of the same concentration produced vastly different signals on the HPLC. Thus, it was likely not appropriate to calculate the concentration of the aqueous stock from standards prepared in methanol. Later, a $100 \mu \mathrm{g} / \mathrm{mL}$ BPA in methanol stock solution was used to prepare all BPA in methanol and water standard solutions and any BPA in water sample solutions to be used for extraction.

Using the gradient mobile phase starting at $90 \%$ methanol to $100 \%$ methanol, the retention time of the BPA peak was determined to be $2.02 \pm 0.03 \mathrm{~min}$, while in water it 
was found to be $2.23 \pm 0.05 \mathrm{~min}$. Typical chromatograms of BPA standards in methanol and water are shown in Figure 3-1through Figure 3-4. Peak area is represented by the yaxis, while the $\mathrm{x}$-axis indicates the time, in minutes. The BPA peak was fairly broad in both solvents, and significantly less sharp in water. Tailing was observed in both solvents. In the figures shown, the blue boxes at either side of the peak indicate the start and end times of the peak, and therefore the start and end times of integration for that peak. Figure 3-1 and Figure 3-2 are typical chromatograms of BPA in methanol. At low concentrations the baseline signal is not as smooth compared to at higher concentrations. In water, the BPA peak is quite jagged as seen in Figure 3-3. At times, the water peak was so broad and messy it didn't really resemble a chromatographic peak at all, shown in Figure 3-4. On a fairly regular basis, the HPLC column was reversed and methanol, acetone and hexane were pumped through to flush any particulate matter or retained analytes out of the column. This procedure helped to improve the signal and resolution, particularly of the BPA in water peak. In almost all cases, the start and end times of the peak needed to be adjusted manually in order to integrate the peak properly.

At concentrations close to the detection limit, the signal was not a smooth line and the software sometimes interpreted signal fluctuations as separate peaks. In these cases the BPA peak was not integrated from start to finish. Instead, the software integrated the peak as multiple parts as indicated by several start and end times, shown in Figure 3-3. If the baseline was still drawn across the bottom of the peak, the individual parts were added to determine the entire peak area. If the baseline was not drawn correctly, the signal-to- noise parameter was adjusted to prevent the software from integrating small 
peaks. Although manual adjustment was needed to obtain integration, the calibration curve indicates that this method was acceptable since the correlation coefficients $\left(R^{2}\right.$ values) were so high in both water and methanol.

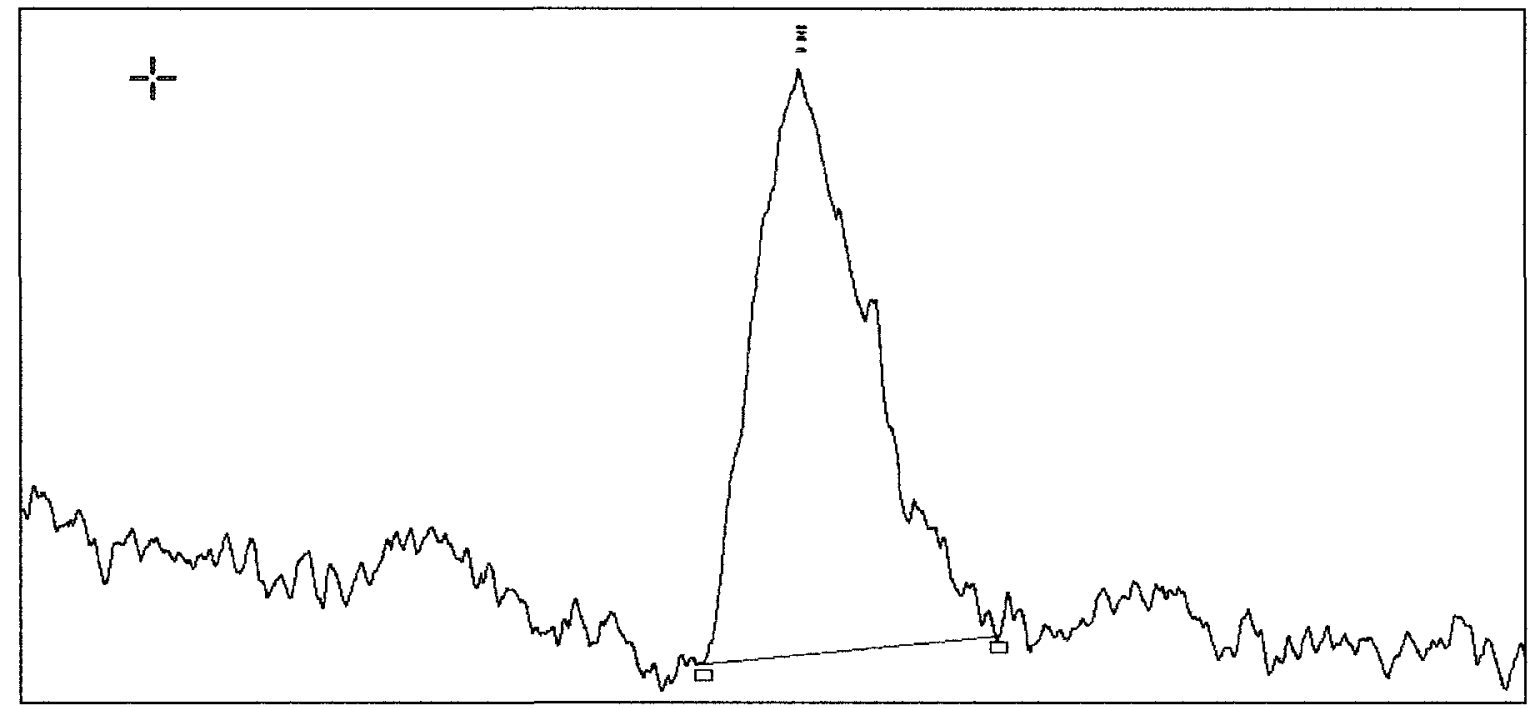

Figure 3-1 HPLC chromatogram of a $50 \mathrm{ng} / \mathrm{mL}$ BPA in methanol standard

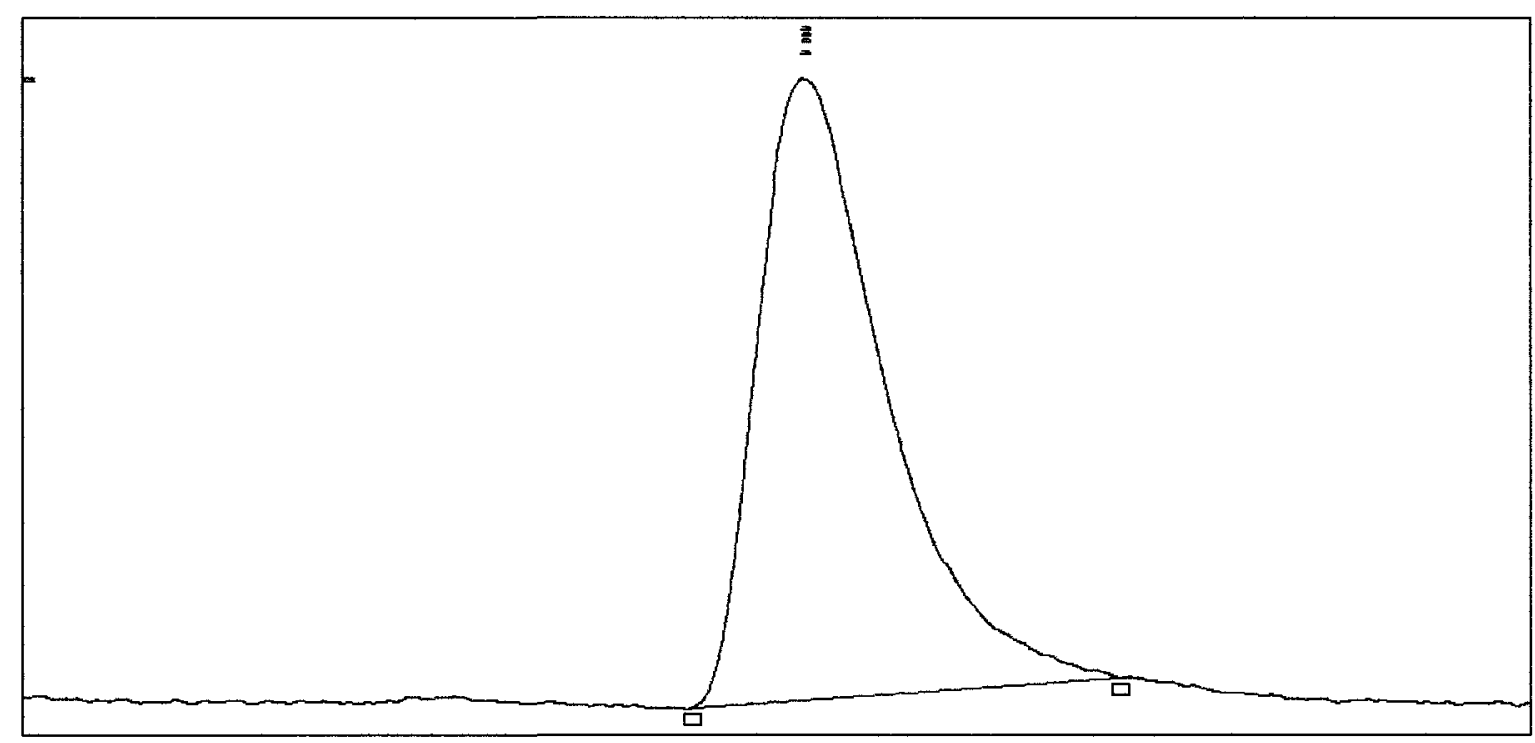

Figure 3-2 HPLC chromatogram of a $1 \mathrm{mg} / \mathrm{mL}$ BPA in methanol standard 


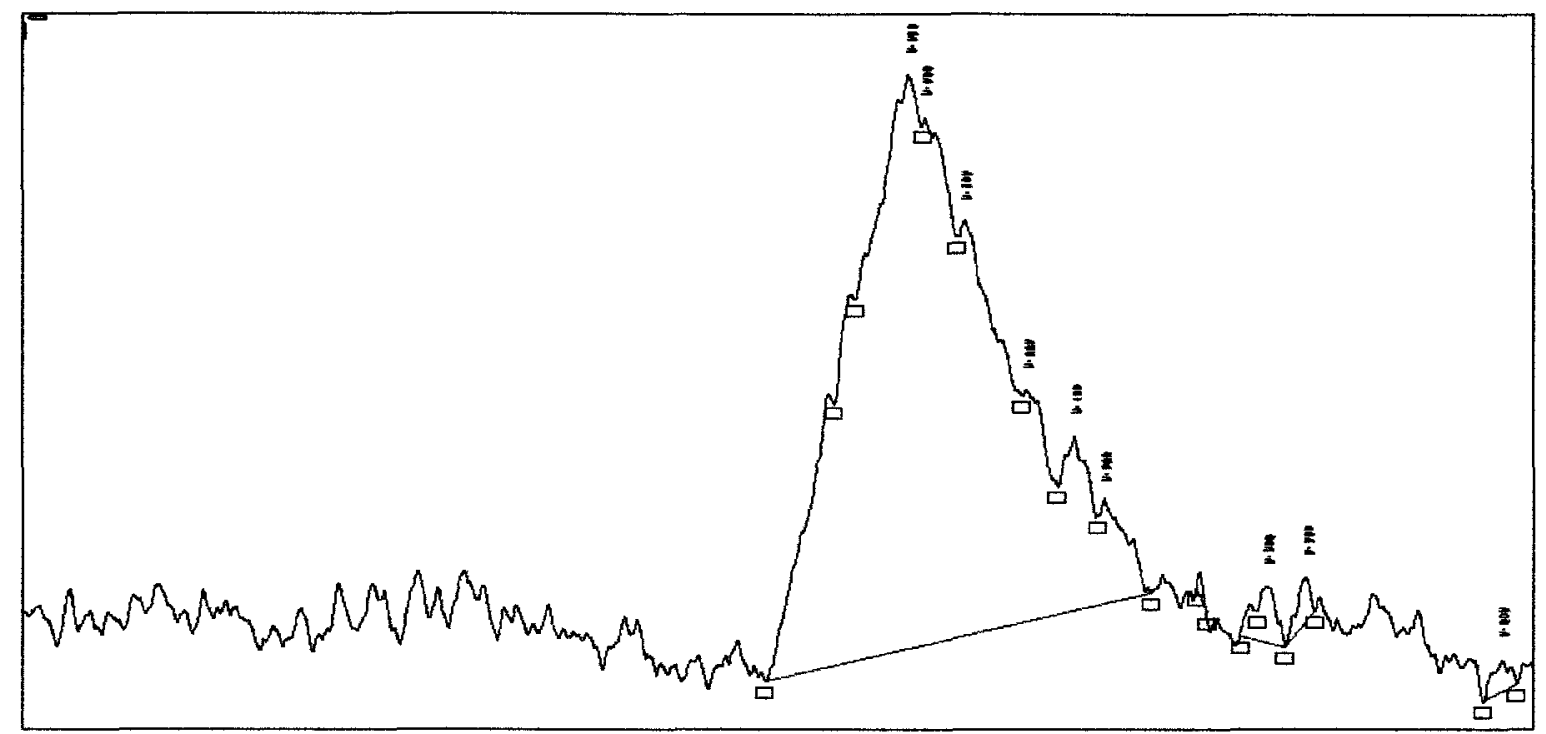

Figure 3-3 HPLC chromatogram of a $100 \mathrm{ng} / \mathrm{mL}$ BPA in water standard

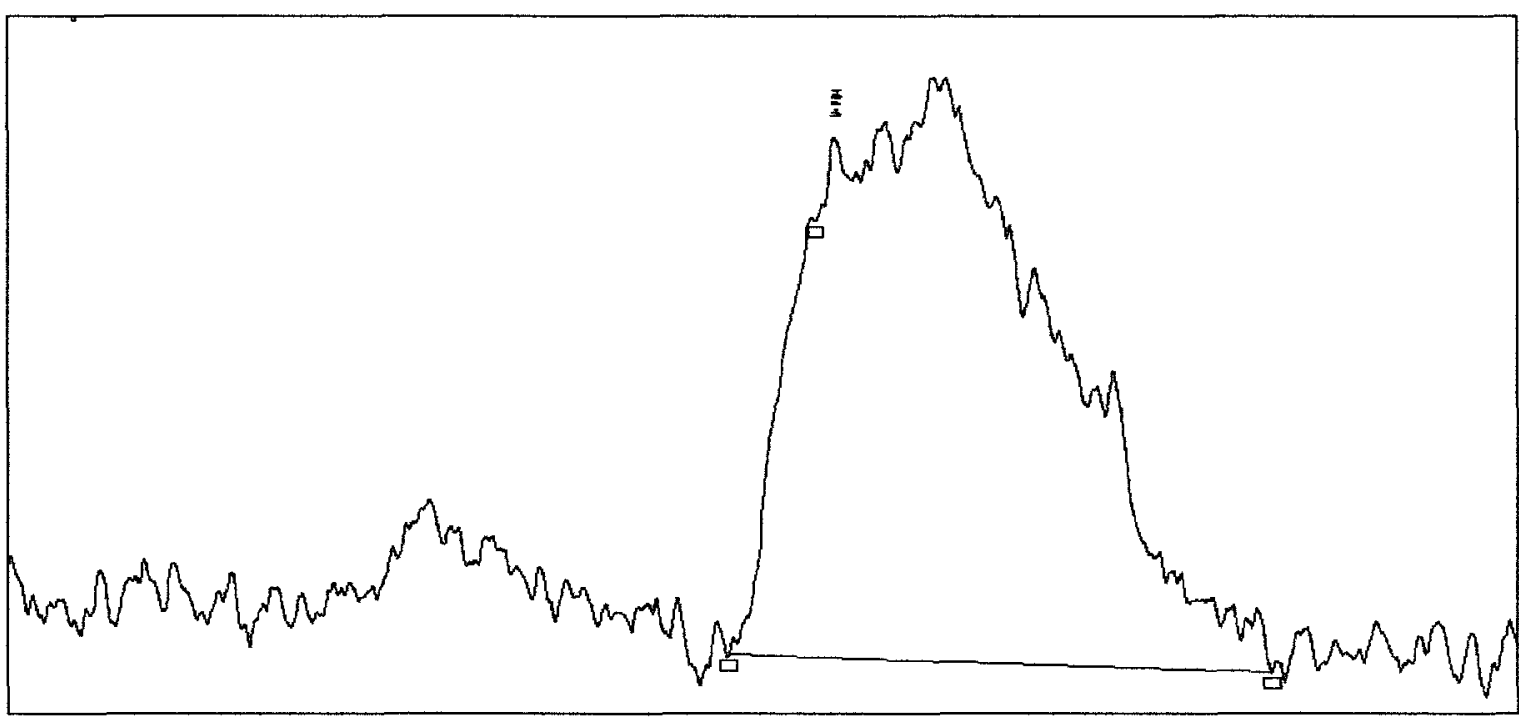

Figure 3-4 HPLC chromatogram of a water solution after BPA extraction using a CNT envelope

To plot BPA calibration curves, several standards of BPA in methanol and water were prepared, ranging in concentration from $5-1000 \mathrm{ng} / \mathrm{mL}$. Each standard was analyzed three times, separated by a methanol run. Three blank runs of pure methanol and deionized water were also analyzed, however no BPA peak was observed in either 
solvent. A calibration curve of peak area versus concentration, shown below in Figure 35, was linear from $10-1000 \mathrm{ng} / \mathrm{mL}$ for BPA in methanol and $25-1000 \mathrm{ng} / \mathrm{mL}$ for BPA in water, with R-squared values of 0.9996 and 0.9994 , respectively. The slope of the calibration curve represents the sensitivity of the instrument. In methanol the sensitivity was determined to be $12045(\mathrm{ng} / \mathrm{mL})^{-1}$, while in water it was found to be $10101(\mathrm{ng} / \mathrm{mL})^{-1}$. The error bars on each point represent the standard deviations from the mean peak area at each concentration. The larger error bars associated with the BPA in water series indicate the difficulties experienced integrating any samples prepared in water and variances resulting from adding several peak areas together.

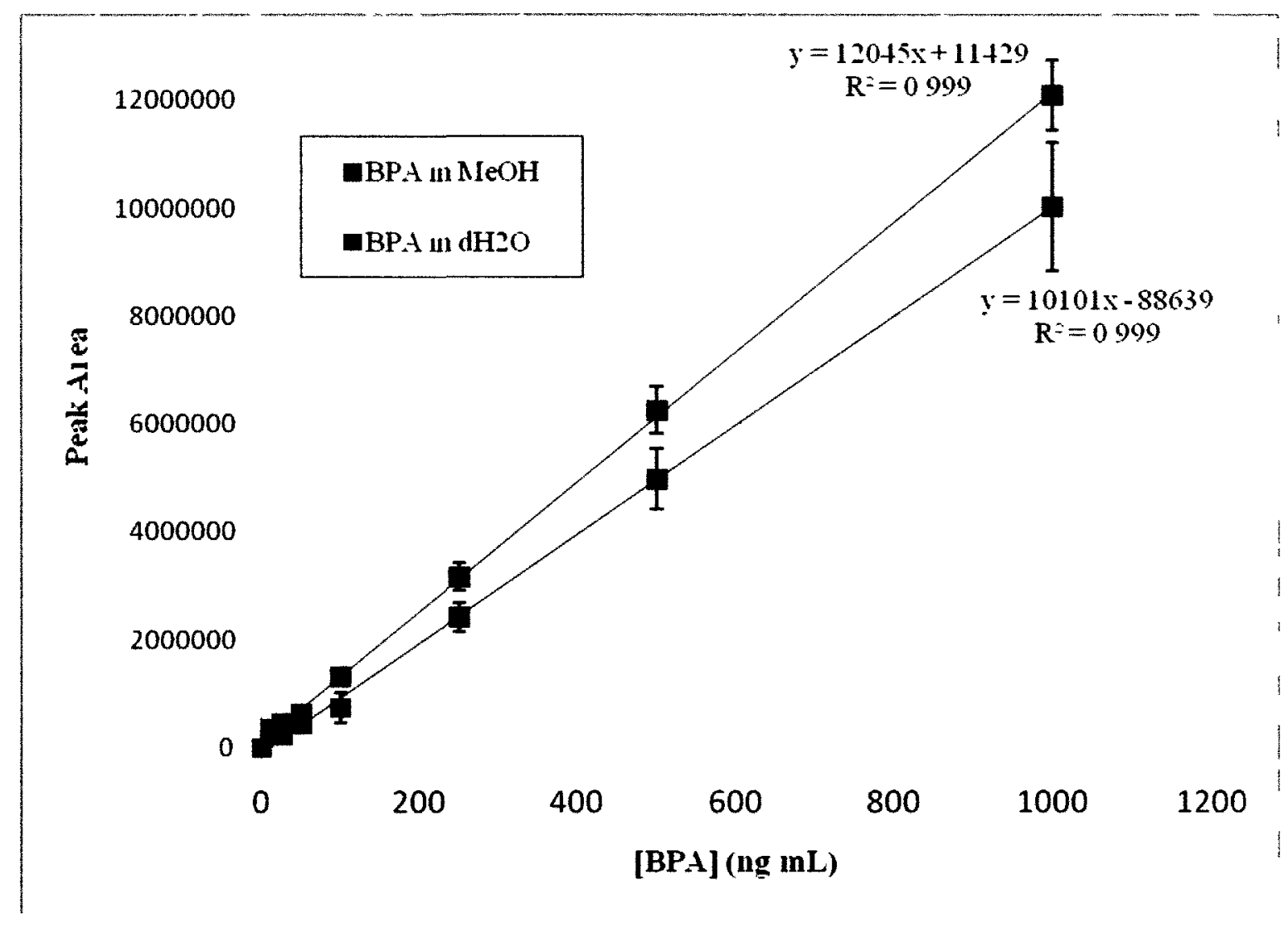

Figure 3-5 Calibration curve of BPA in methanol (red) and BPA in water (blue). 
The limit of detection (LOD) and limit of quantitation (LOQ) are usually calculated using the formulae:

$L O D=\frac{3 \sigma_{B}}{b}$ and $L O Q=\frac{10 \sigma_{B}}{b}$

where $\sigma_{B}$ is the standard deviation of the blank and $\mathrm{b}$ is the sensitivity of the instrument. However, since no peaks could be integrated in the blank methanol and water chromatograms, the limits of detection and quantitation could not be calculated. Thus the limit of detection was denoted as the lowest detectable concentration, which was 10 $\mathrm{ng} / \mathrm{mL}$ in methanol and $25 \mathrm{ng} / \mathrm{mL}$ in water.

\subsection{BPA Solubility}

The solubility of BPA in water was measured over a range of temperature and $\mathrm{pH}$ conditions. To determine the solubility of BPA in water, solid BPA was combined with water in a glass vial until the water was saturated, indicated by the presence of undissolved BPA in the vial. The temperature of the experiment was controlled by placing the vial in a water bath. Periodically, a minute aliquot of the supernatant was removed and diluted with methanol. The concentration of the solution was determined from a calibration curve of BPA in methanol. The concentration of the solution was measured over the course of a day.

Solubility is strongly affected by temperature and so the concentration of was measured over a broad temperature range, $5-45^{\circ} \mathrm{C}$. This temperature range was chosen since it reflects environmental conditions. When a solute dissolves in aqueous solution, energy is 
required to break the solute-solute and solvent-solvent bonds to form solute-solvent bonds. Generally, organic analytes are more soluble in aqueous solutions at higher temperatures due to the increase in energy provided to break the solute-solute bonds. This is true for BPA as well. The solubility of BPA at $45^{\circ} \mathrm{C}$ was almost 5 times greater than at $5^{\circ} \mathrm{C}$. Furthermore, BPA solubility will greatly be affected by the $\mathrm{pH}$ of the aqueous solution. BPA has two $-\mathrm{OH}$ groups which are deprotonated at basic pHs, 9.59 and 11.30. Thus, BPA solubility in aqueous solution was measured over a wide $\mathrm{pH}$ range, $\mathrm{pH} 2.01-12.63$, at $25^{\circ} \mathrm{C}$. Above the second $\mathrm{pKa}$, $\mathrm{BPA}$ was found to be considerably more soluble in water since it is more stable in aqueous solution when deprotonated. Figure 3-6 illustrates BPA solubility over time for all the temperature and pH conditions discussed. 


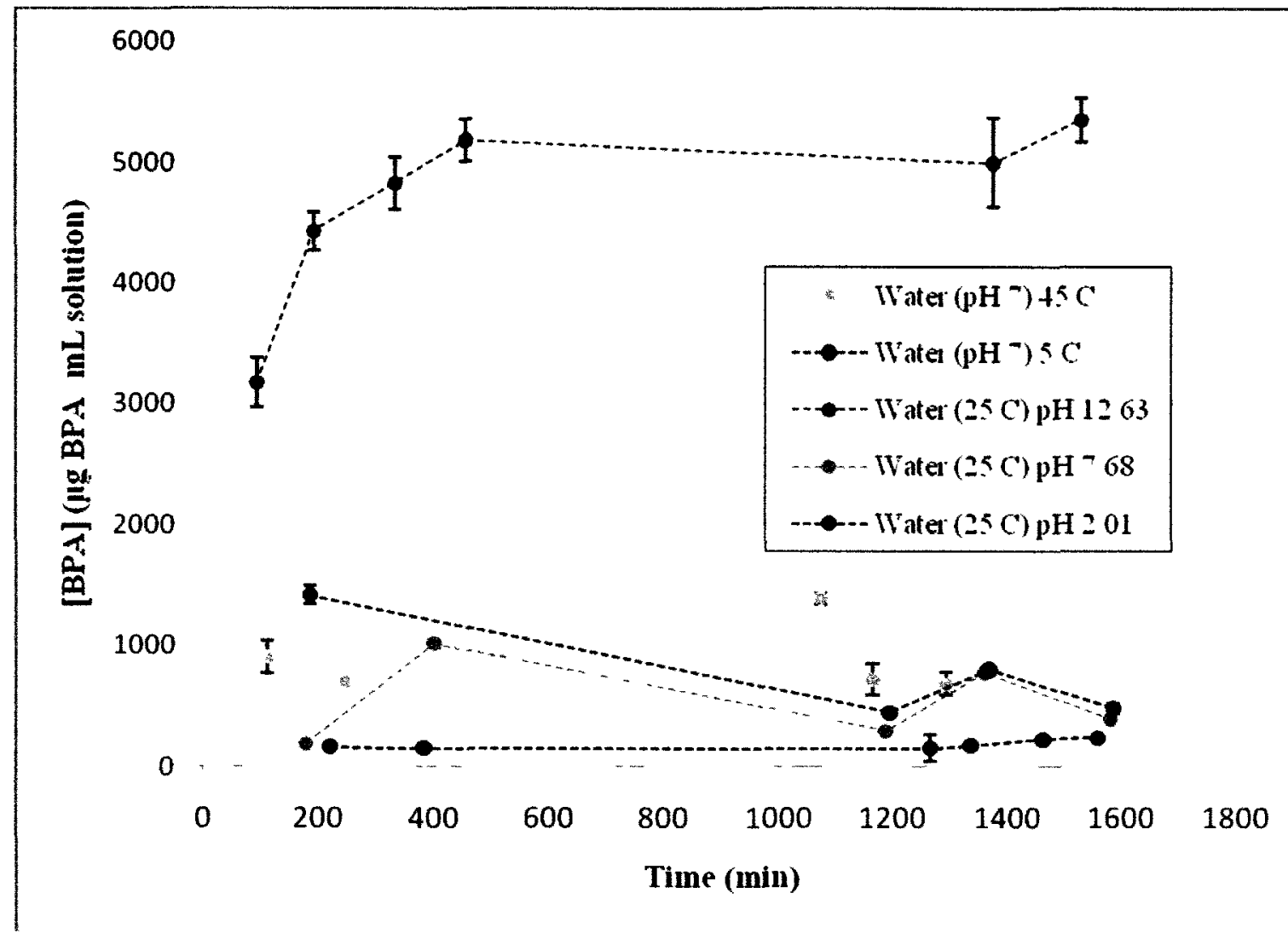

Figure 3-6 Concentration of BPA in aqueous solution over a wide range of temperature and $\mathrm{pH}$ conditions.

BPA solubility in common organic solvents was also measured experimentally by the same procedures as BPA solubility in water. The solubility in each solvent was measured over the course of several days, although equilibrium was reached before even the first aliquot was removed from all solvents, as shown in Figure 3-7. Since BPA solubility is so many orders of magnitude greater in methanol and acetone compared to the other solvents, the data in the $\mathrm{ng} / \mathrm{mL}$ and $\mu \mathrm{g} / \mathrm{mL}$ range could not be accurately displayed using the scale shown in Figure 3-7. Therefore a table of the average concentration of the BPA solutions has been listed in Table 3-1. 


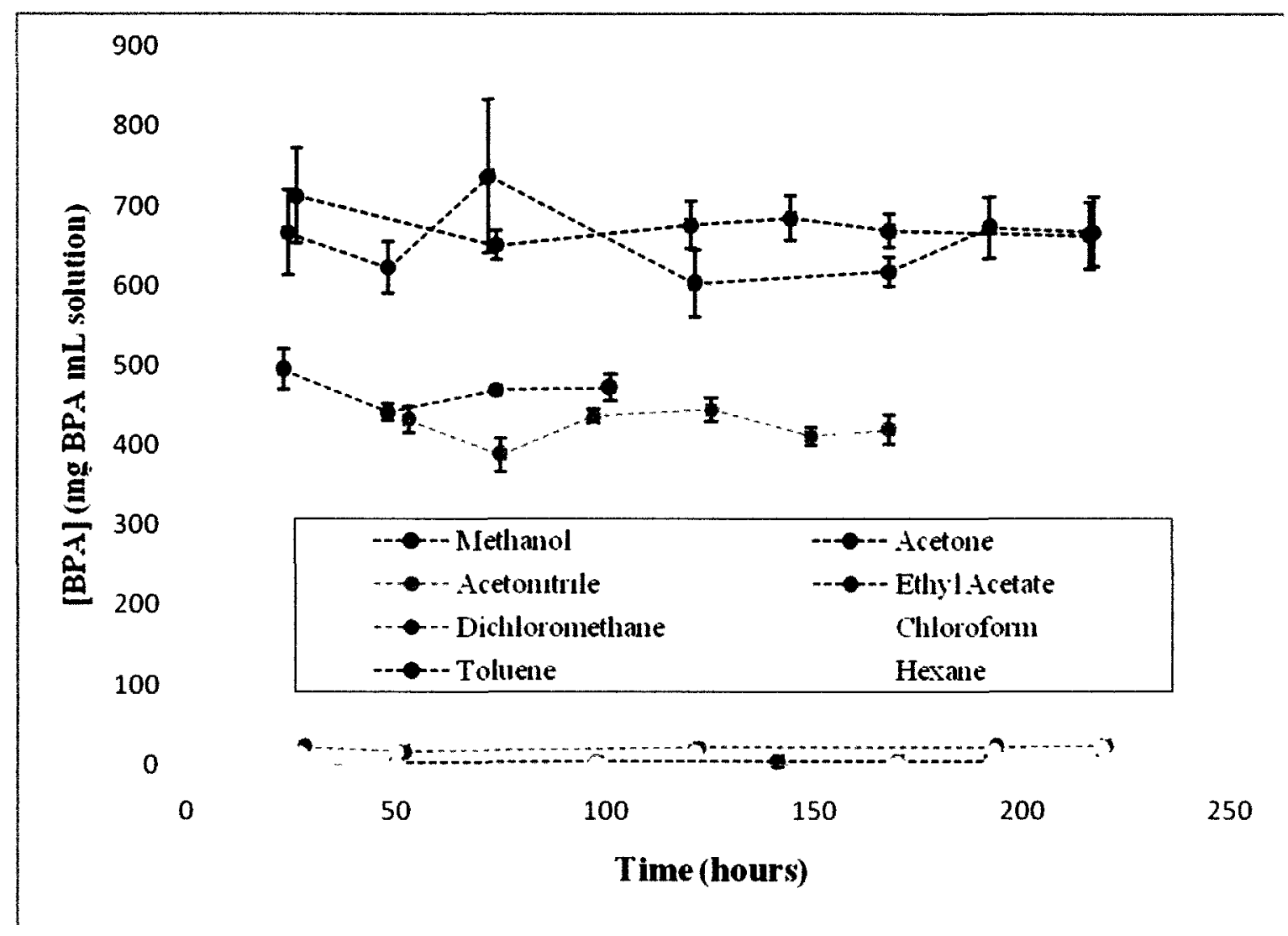

Figure 3-7 Concentration of BPA in organic solvents ranging in polarity.

It can be seen in Figure 3-7 that BPA is extremely soluble in relatively polar solvents, such as methanol and acetone, and not very soluble in non-polar solvents, including toluene and hexane. According to the principle "like dissolves like" a solute will dissolve in a solvent that has similar chemical characteristics to it. In terms of polarity, polar solutes will be soluble in polar solvents and the same is true for non-polar solutes and solvents. Dipole moment $(\mu)$, the measure of net charge in a molecule, can be used to quantify the polarity or "solvent strength" of a given solvent. The greater the dipole moment, the more polar a molecule is. The dipole moments of each of the solvents used to measure BPA solubility, including water are shown in Figure 3-8 in addition to the average concentration of BPA in each of these solutions. When the dipole moment of 
each solvent is compared to the solubility of BPA in that solvent, a trend immerges. With a few exceptions, as the dipole moment increases, so does the solubility of BPA.

Table 3-1 Comparsion between the solubility of BPA and dipole moment of the solvent

\begin{tabular}{|c|c|c|}
\hline & $\begin{array}{c}\text { BPA solubility (mg/L } \\
\text { solution) }\end{array}$ & Dipole Moment, $\boldsymbol{\mu}$ (debyes) \\
\hline Hexane & 60.5 & 0 \\
\hline Toluene & $3.98 \times 10^{3}$ & 0.37 \\
\hline Chloroform & $1.73 \times 10^{4}$ & 1.05 \\
\hline Dichloromethane & $2.10 \times 10^{4}$ & 1.54 \\
\hline Methanol & $6.55 \times 10^{5}$ & 1.69 \\
\hline Ethyl Acetate & $4.68 \times 10^{5}$ & 1.78 \\
\hline Water & 533 & 1.85 \\
\hline Acetone & $6.78 \times 10^{5}$ & 2.91 \\
\hline Acetonitrile & $4.21 \times 10^{5}$ & 3.92 \\
\hline
\end{tabular}

In order to determine what polar character (extremely non-polar to extremely polar) is most similar to the polarity of BPA; the solubility of BPA in the solvents listed can be plotted against the dipole moment of the corresponding solvent as shown in Figure 3-8. Generally, as dipole moment (and thus polarity) increases, so does the solubility of BPA. The major exception is the solubility of BPA in water. BPA is most soluble in acetone and methanol, which both possess polar, hydrophilic functionalities but also contain organic character. Similarly, the extremities of BPA have polar, hydrophilic character, but the bulk of the molecule is organic. Therefore, when BPA dissolves in acetone or methanol, the matching polar and organic groups can align to maximize the "like dissolves like" rule. Furthermore, BPA is synthesized from the condensation of phenol 
with acetone. Thus the chemical structures of acetone and methanol are most similar to that of BPA compared to the other studied solvents. Although water is more polar than methanol, it does not contain any organic functional groups and thus is only similar to BPA at either end. Furthermore, the strong hydrogen bonds between water molecules make it difficult for BPA to "insert" into liquid water, even though water is polar. Therefore, if water is excluded, it can generally be said the more polar a solvent is the more likely will dissolve BPA.

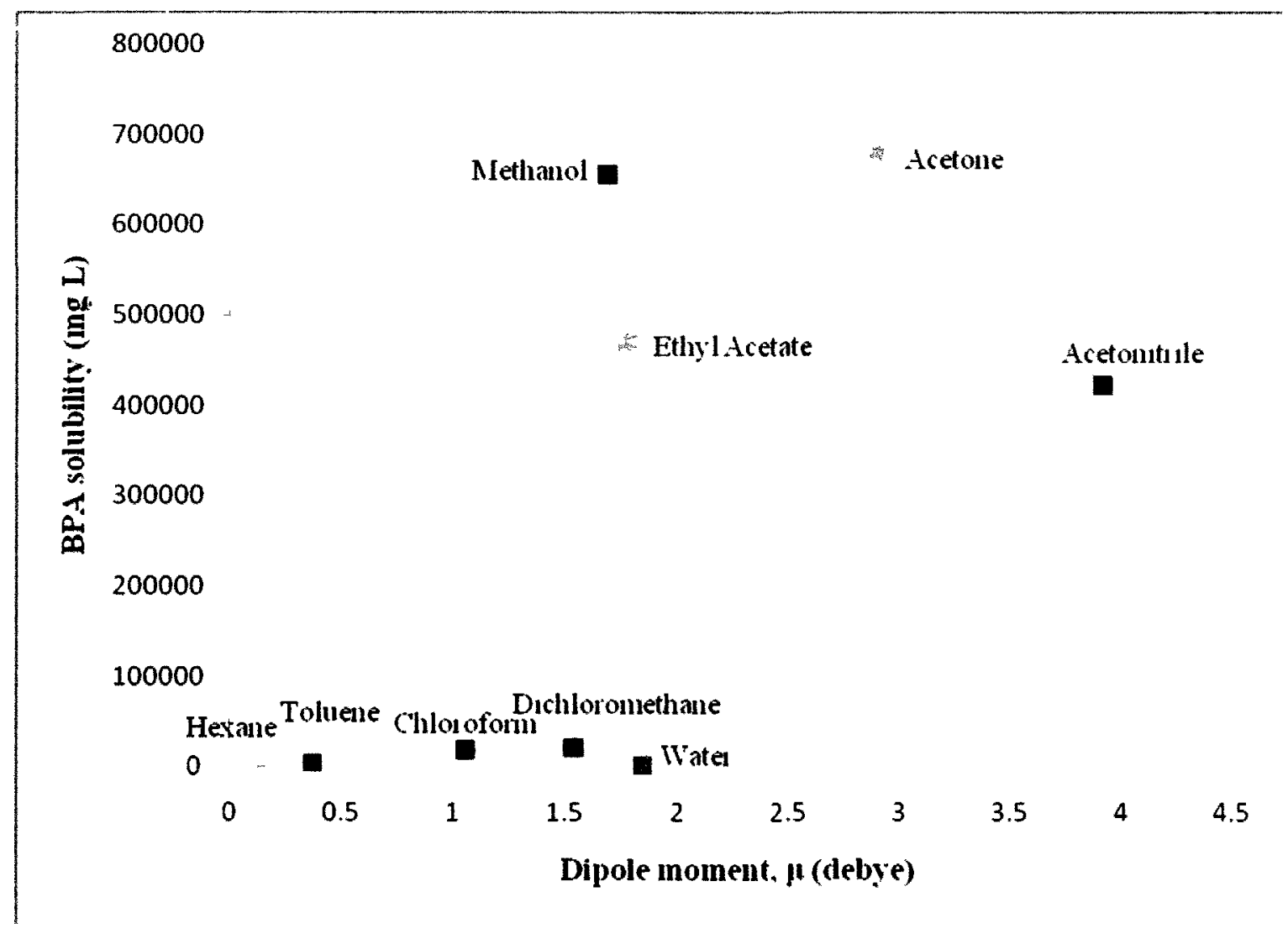

Figure 3-8 Comparsion of BPA solubility to dipole moment, which reflects the degree of polar character the solvent has. 


\subsection{Carbon Nanotube Characterization}

The specific surface areas of pristine single, double and multi-walled carbon nanotubes were determined using the BET method by nitrogen adsorption at 77K. These measurements were provided by Dr. Mohamed Abdel Salam at King Abdulaziz University, Jeddah, Saudi Arabia. A table of the CNTs used and their measured specific surface areas is shown below. It can be seen that specific surface area increases with smaller nanotube diameter. The surface area measurements were obtained from a different research group who did not have samples of the oxidized single and multiwalled nanotubes, and thus the specific surface areas of the oxidized CNTs are not known.

Table 3-2 Specific surface area measurements of the carbon nanotubes used in this work.

\begin{tabular}{|c|c|c|c|}
\hline & Diameter $(\mathbf{n m})$ & Length $(\boldsymbol{\mu m})$ & $\begin{array}{c}\text { Specific Surface } \\
\text { Area }\left(\mathbf{m}^{2} / \mathbf{g}\right)\end{array}$ \\
\hline SWCNTs & $<2$ & $5-15$ & 375 \\
\hline DWCNTs & $<5$ & $5-15$ & 417 \\
\hline L-MWCNTs 10 & 10 & $5-15$ & 220 \\
\hline L-MWCNTs 1020 & $10-20$ & $5-15$ & 69 \\
\hline S-MWCNTs 1020 & $10-20$ & $1-2$ & 116 \\
\hline
\end{tabular}

Transmission electron microscopy (TEM) was used to characterize some of the pristine CNTs used in this work. The TEM images were also provided by provided by Dr.

Mohamed Abdel Salam at King Abdulaziz University, Jeddah, Saudi Arabia who did not have access to the oxidized CNTs and thus they could not be characterized by transmission electron microscopy. A TEM image of the single-walled carbon nanotubes 
is shown in Figure 3-9. This micrograph indicates the outer diameter of the tubes is roughly $5 \mathrm{~nm}$, while the inner diameter is about $2 \mathrm{~nm}$. The electron micrograph displayed in Figure 3-10 displays the dimensions of a long-MWCNT, which has a supposed diameter of $10 \mathrm{~nm}$, but is shown to be wider in the TEM image.

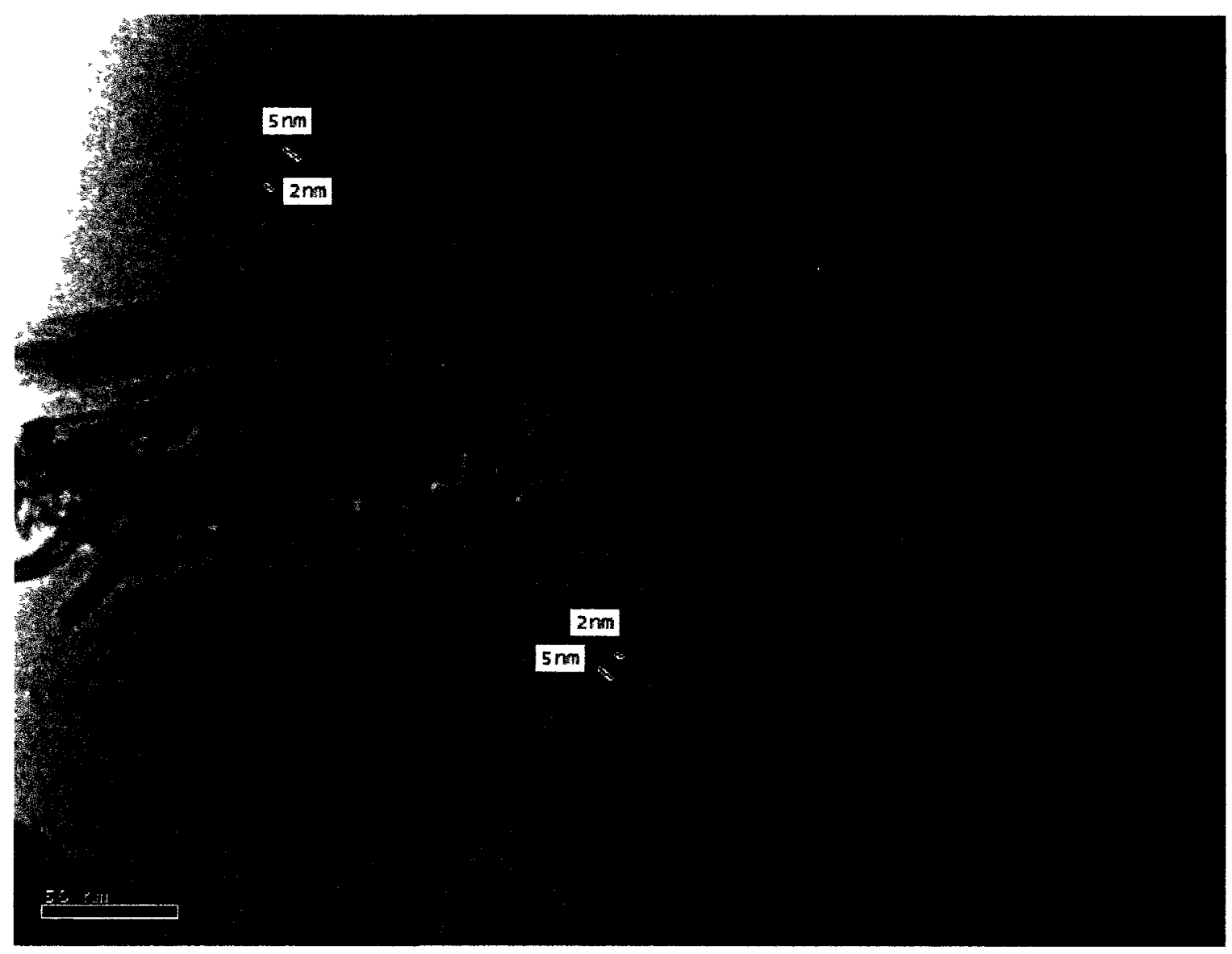

Figure 3-9 TEM image of SWCNTs. 


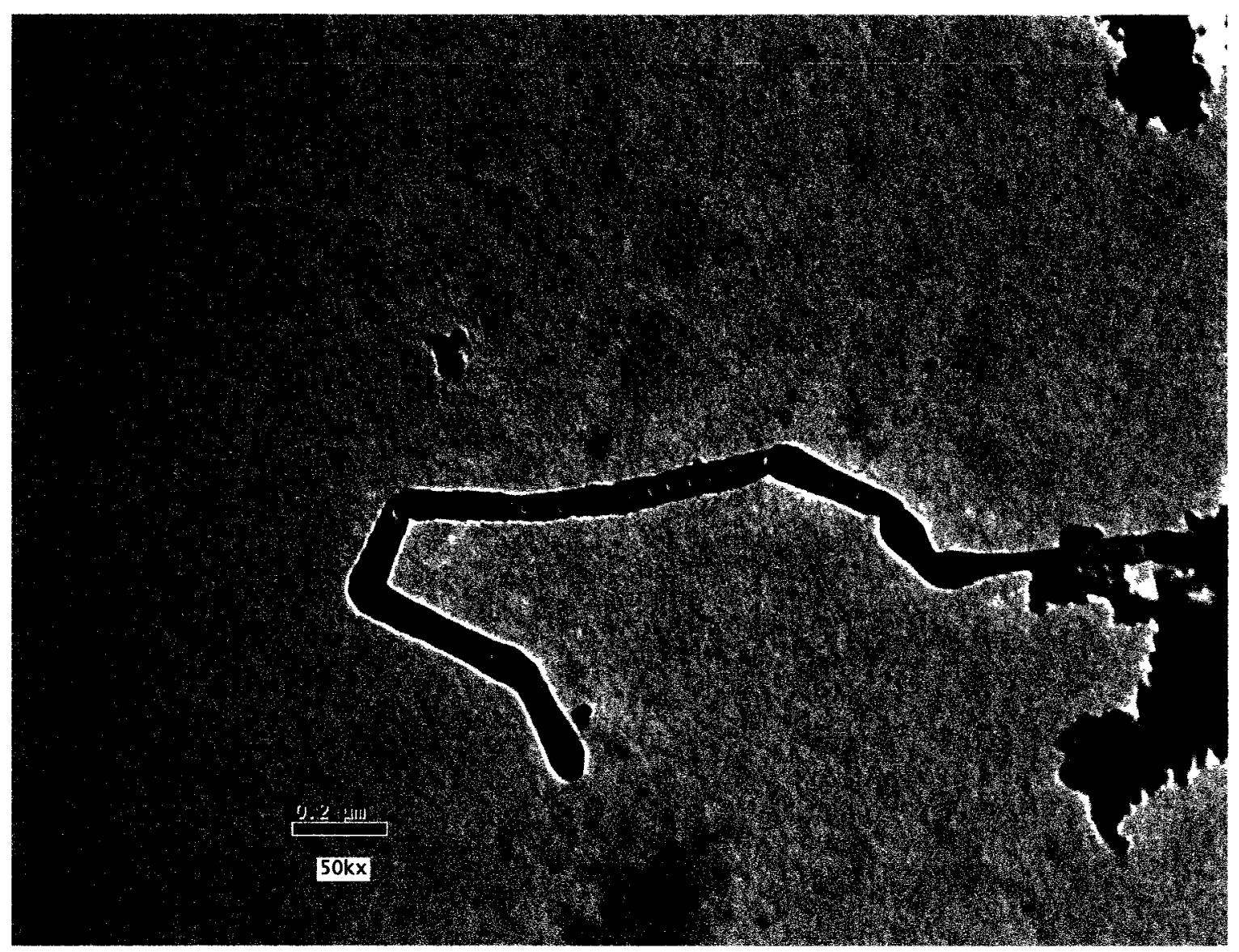

Figure 3-10 TEM image of long MWCNTs with a diameter of $10 \mathrm{~nm}$ as stated by the manufacturer.

Infrared spectroscopy (IR) was used to confirm the formation of oxygen-containing functional groups on the surface of the carbon nanotubes after oxidation. Spectra of both pristine and oxidized carbon nanotubes (solid) were obtained for comparison. Figure 311 reveals the IR spectra of pristine and oxidized SWCNTs. The aromatic $\mathrm{C}=\mathrm{C}$ is represented in Figure 3-11 by the fairly strong peak observed at $\sim 1629 \mathrm{~cm}^{-1}$ and the smaller, sharper peak at $\sim 1460 \mathrm{~cm}^{-1}$, which are in agreement with previously reported literature values [99]. Aromatic carboxylic acid groups are generally characterized by the aromatic $\mathrm{C}=\mathrm{O}$ vibration at $\sim 1730 \mathrm{~cm}^{-1}$. No peaks were displayed in either spectra for the 
pristine or oxidized nanotubes at this stretch. Similarly, the aromatic $C=C$ stretch was easily recognized on the spectra of pristine and oxidized MWCNTs shown in Figure 312 , while peaks representing the aromatic $\mathrm{C}=\mathrm{O}$ stretch were absent. The large broad peak displayed in all spectra at $-3400 \mathrm{~cm}^{-1}$ is characteristic of an $\mathrm{O}-\mathrm{H}$ stretch. While the carboxylic acid or alcohol functionalities created upon oxidation may be represented by this stretch, it is most likely due to water contamination from moisture, as it is also present in the IR spectrum of a $\mathrm{KBr}$ pellet (not shown). Oxidative processes used to functionalize carbon nanotubes have been published involving dilute or concentrated acids. In this work, dilute $\mathrm{HNO}_{3}$ was used to oxidize the CNTs. If the degree of oxidation (ie. the concentration of $-\mathrm{COOH}$ groups) was low from the dilute acid, it is possible the effects of oxidation may not be seen by IR spectroscopy.

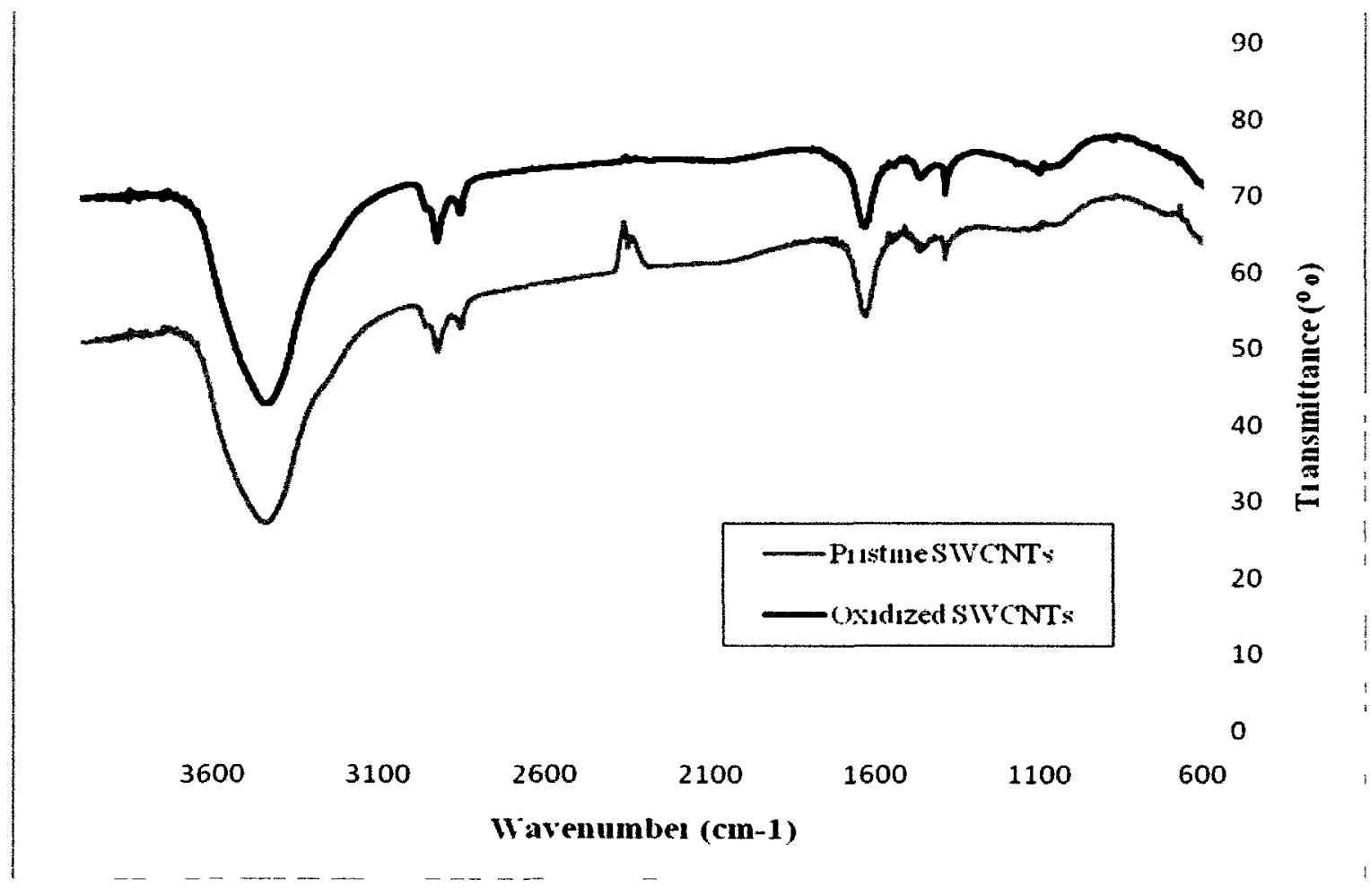

Figure 3-11 Infrared spectra of pristine (blue) and oxidized (red) SWCNTs. 


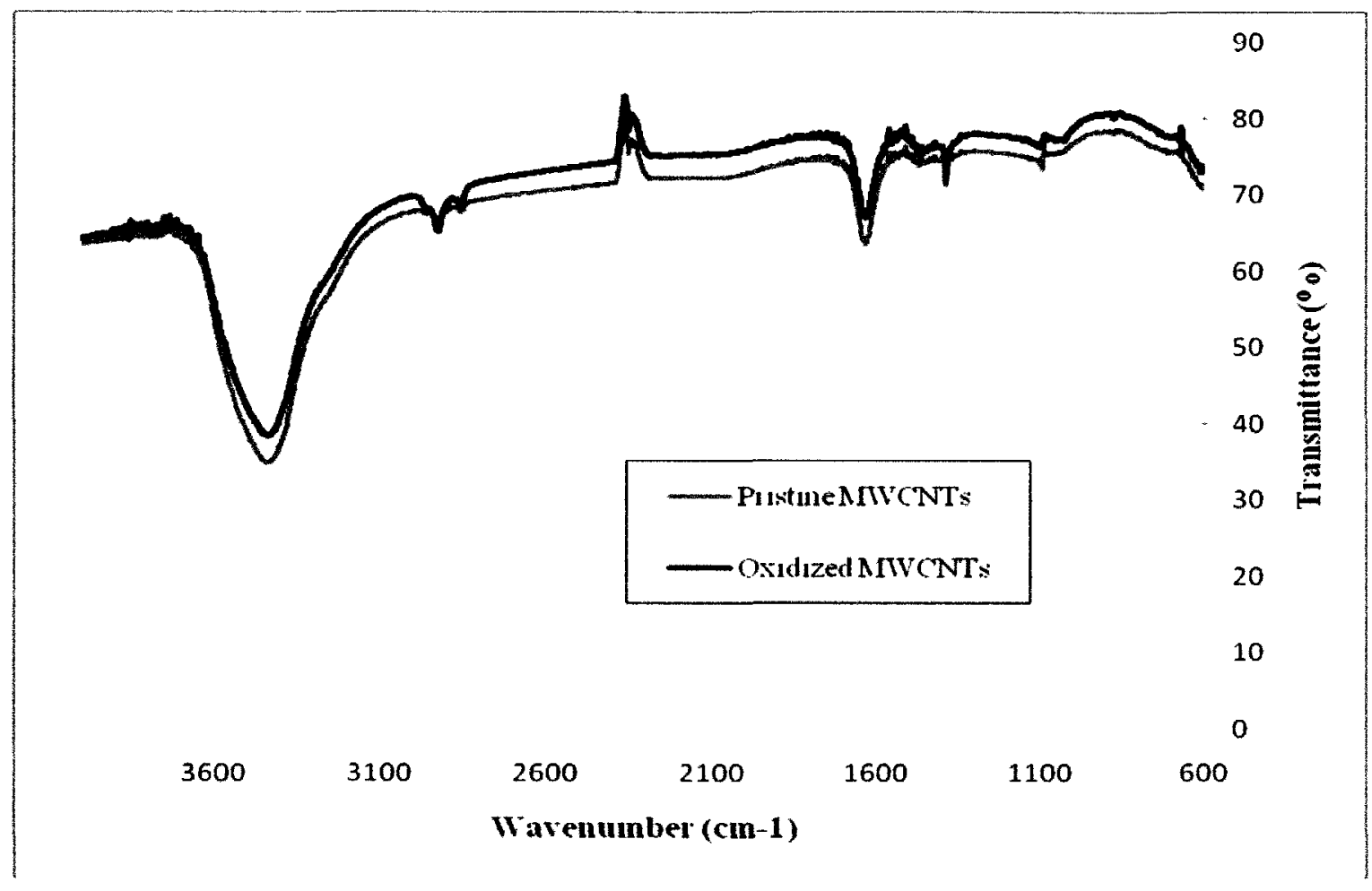

Figure 3-12 Infrared spectra of pristine (blue) and oxidized (red) MWCNTs.

Scanning electron microscopy (SEM) was also used to visually characterize the pristine and oxidized CNTs by examining the nanotube surface and dimensions. Electron micrographs of the pristine and oxidized SWCNTs are shown in Figure 3-13 and Figure $3-14$, respectively. Although the manufacturer indicated the pristine CNTs were less than $2 \mathrm{~nm}$ in diameter and 5-15 $\mu \mathrm{m}$ in length, the nanotubes shown in either image are tens of nanometers in diameter and several microns in length. Although CNT shortening has been reported upon oxidation in strong acids, no observable changes in length or diameter are visible when pristine and oxidized nanotubes are compared. Similarly, Figure 3-15 and Figure 3-16 display pristine and oxidized MWCNTs. The pristine CNTs were said to be $10 \mathrm{~nm}$ in diameter and 5-15 $\mu \mathrm{m}$ in length. While some nanotubes shown in either 
image may have diameters as thin as $10 \mathrm{~nm}$, the majority of tubes were several tens of nanometers in diameter. The lengths appear to be several microns in both images. 


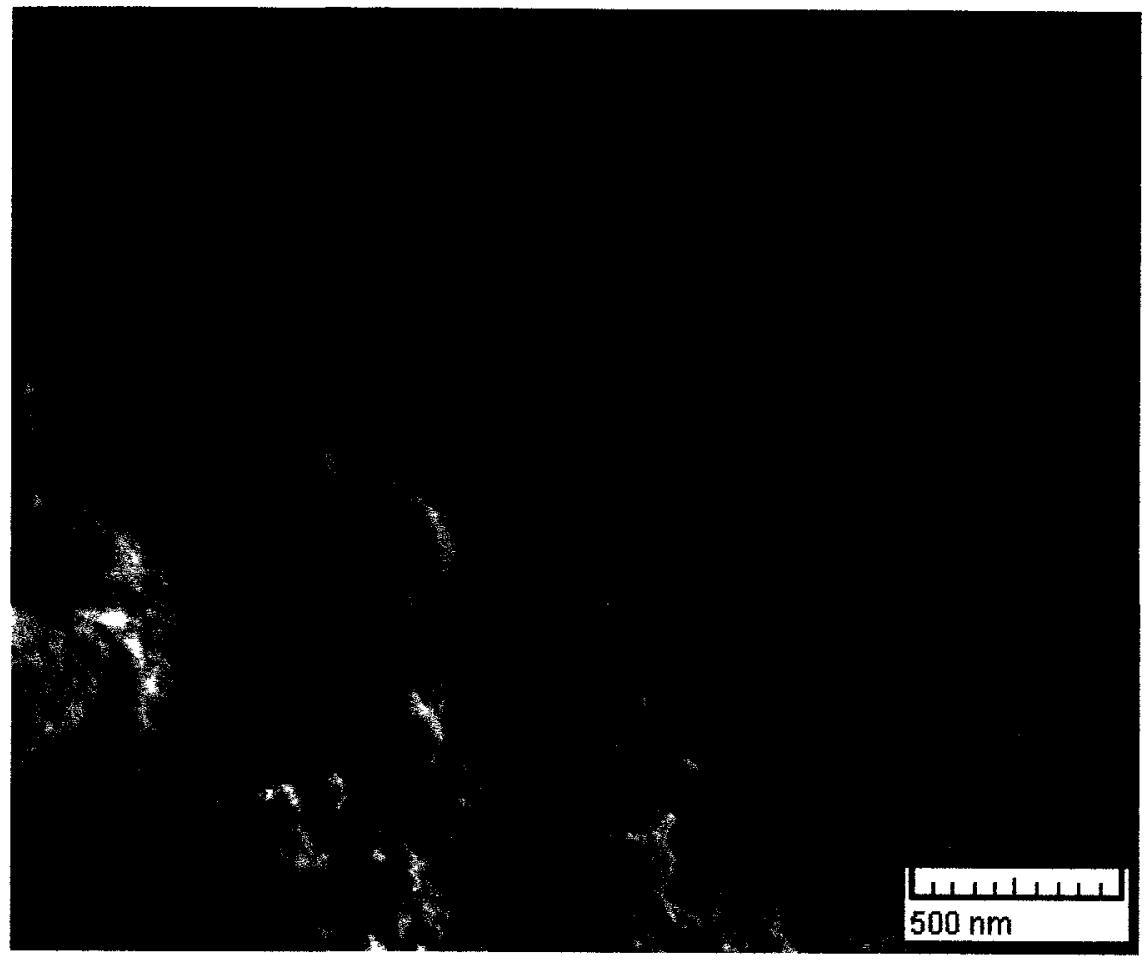

Figure 3-13 SEM image of pristine SWCNTs.

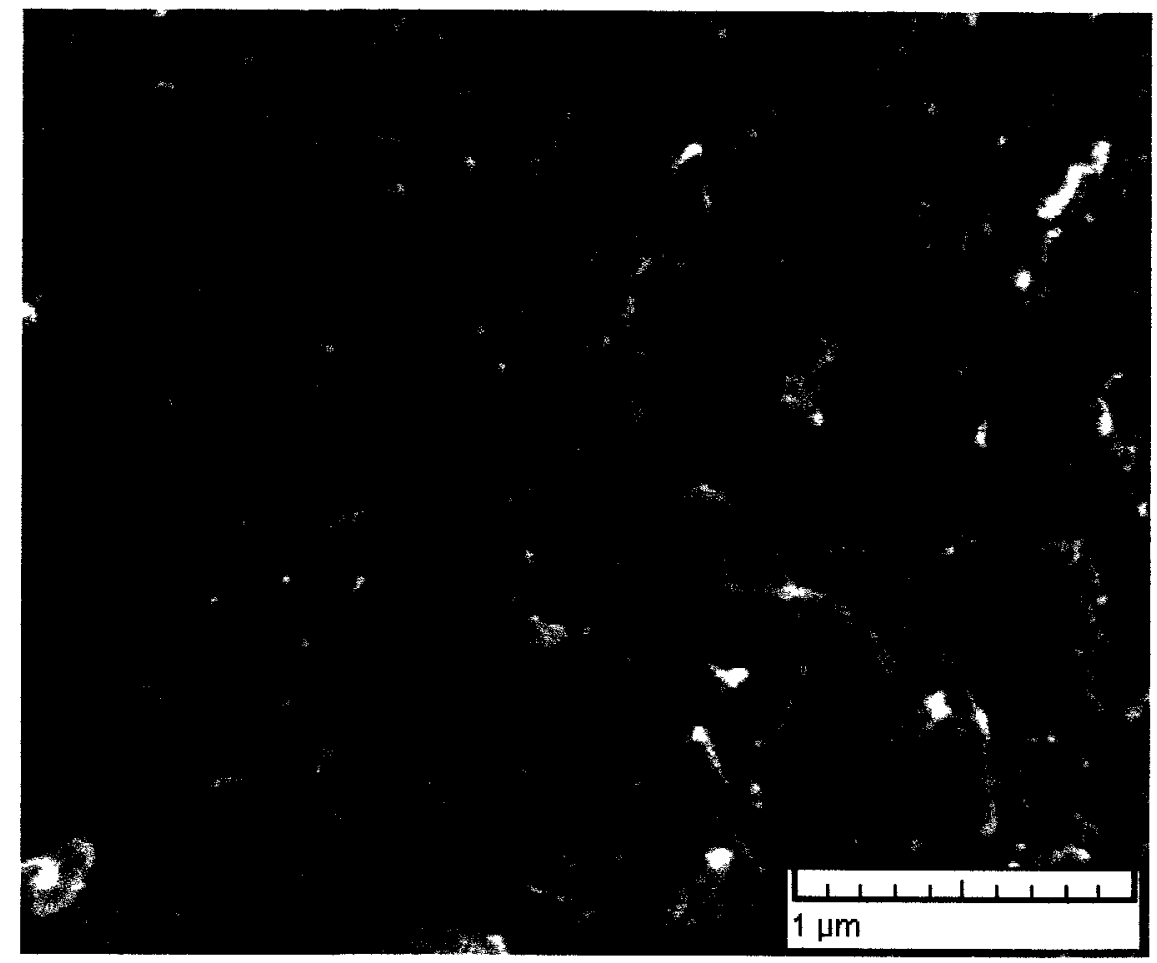

Figure 3-14 SEM image of oxidized SWCNTs. 


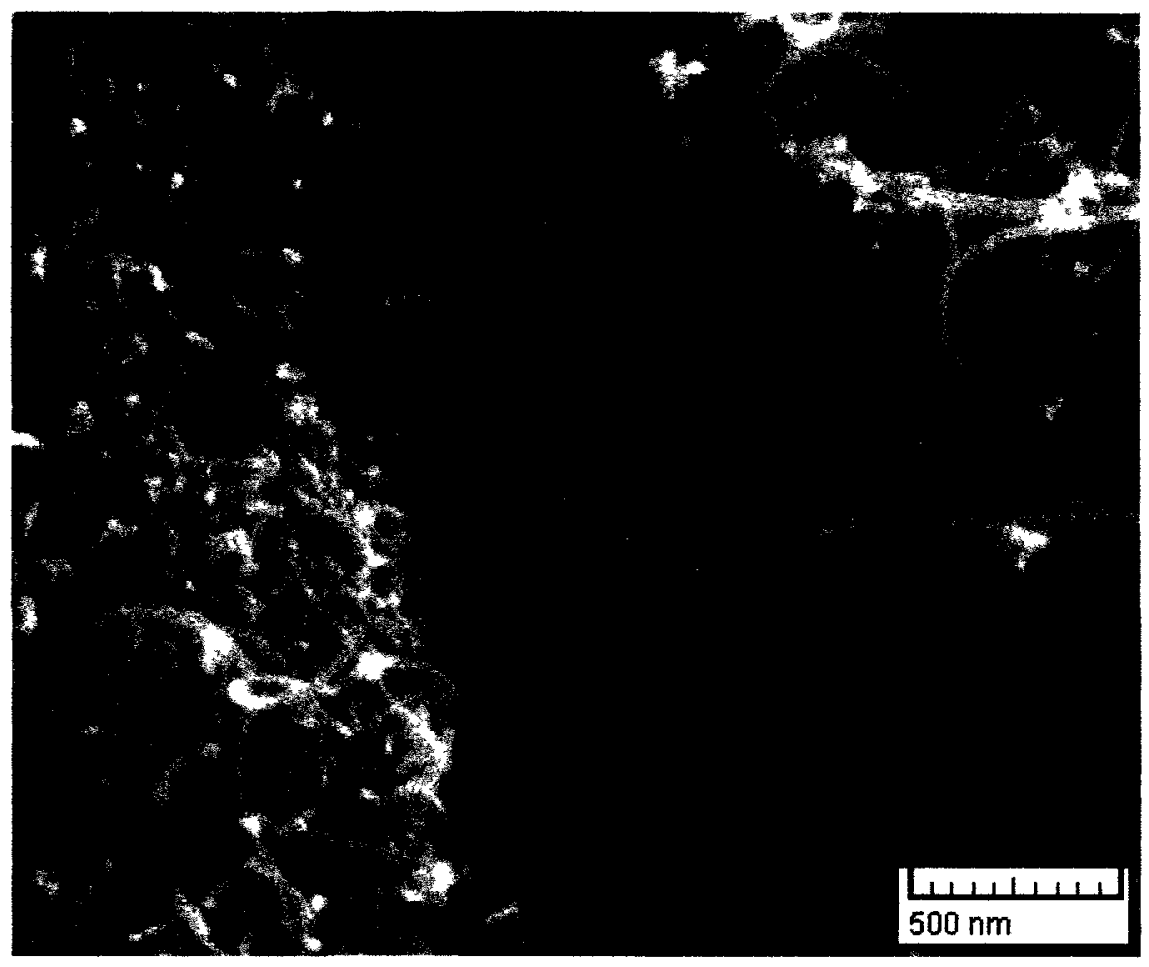

Figure 3-15 SEM image of pristine MWCNTs.

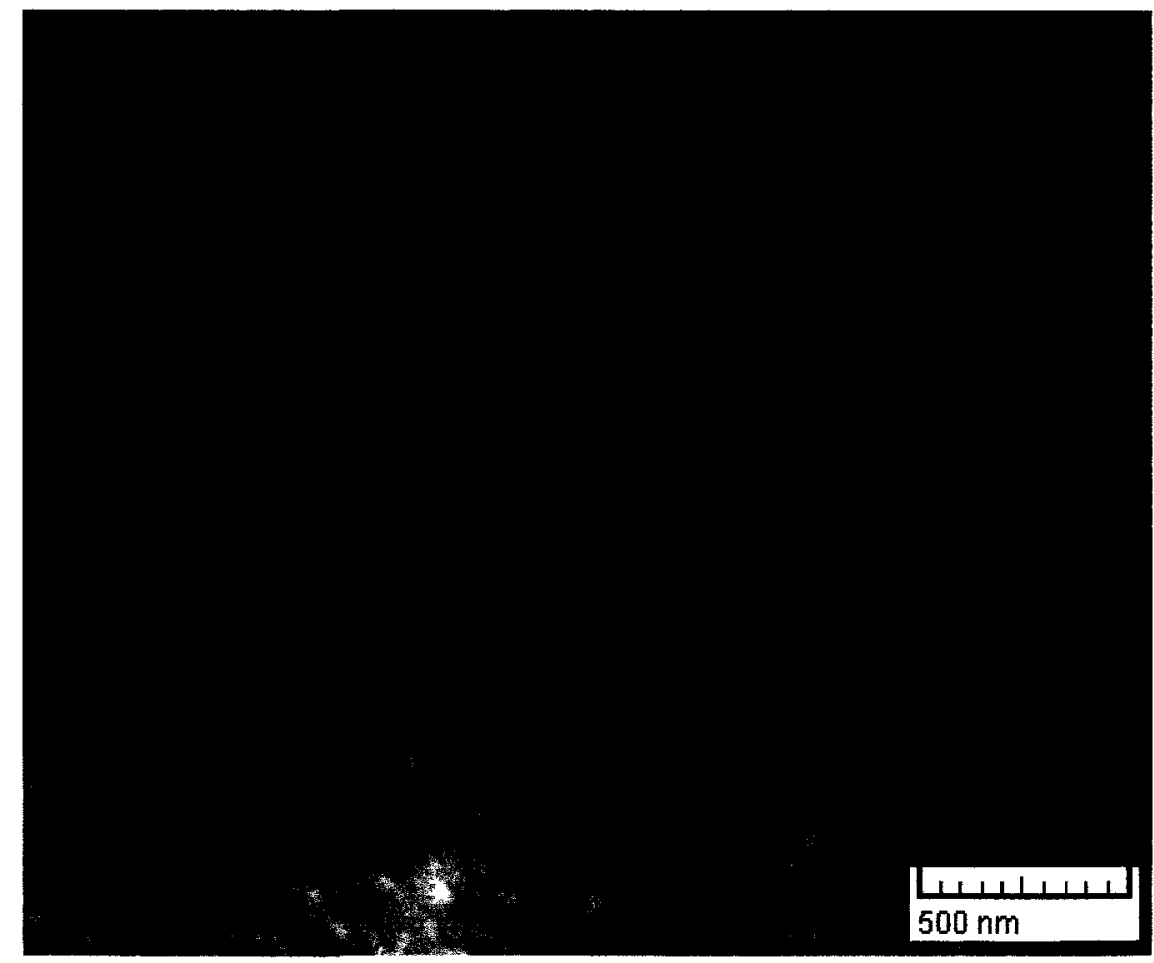

Figure 3-16 SEM image of oxidized MWCNTs. 
Salam and Burk [100] visually characterized aqueous suspensions of pristine and oxidized CNTs to confirm the presence of acidic functional groups upon oxidation. CNTs were treated by three different oxidation techniques. Aqueous suspensions of each of these oxidized tubes, as well as pristine MWCNTs were prepared 6 months in advance to characterization. A photograph of the suspensions after 6 months indicated that pristine MWCNTs would settle out of solution since they were insoluble in water, while those oxidized in $\mathrm{HNO}_{3}$ were so significantly soluble the black suspension was completely opaque. The same type of characterization technique has been demonstrated in this study to confirm CNT oxidation for both the single and multi-walled CNTs. Suspensions of pristine and oxidized SWCNTs and MWCNTs were prepared and sonicated for 30 minutes. After sonication, the solutions were left for several days and then photographed to compare the suspensions. Figure 3-17 indicates that the acid-treated CNTs were oxidized. In the image, the suspensions have been split in two pairs, SWCNTs shown on left and MWCNTs shown on the right. Within each pair, the suspension of pristine CNTs in water is shown on the left and the aqueous suspension of oxidized CNTs is displayed on the right. Likewise to the image shown by Salam and Burk [100], the pristine CNTs settled out of solution, while the oxidized CNTs were completely suspended. The dramatic differences between the pristine and oxidized solution for either type of nanotube confirm the existence of oxygen-containing functional groups. 


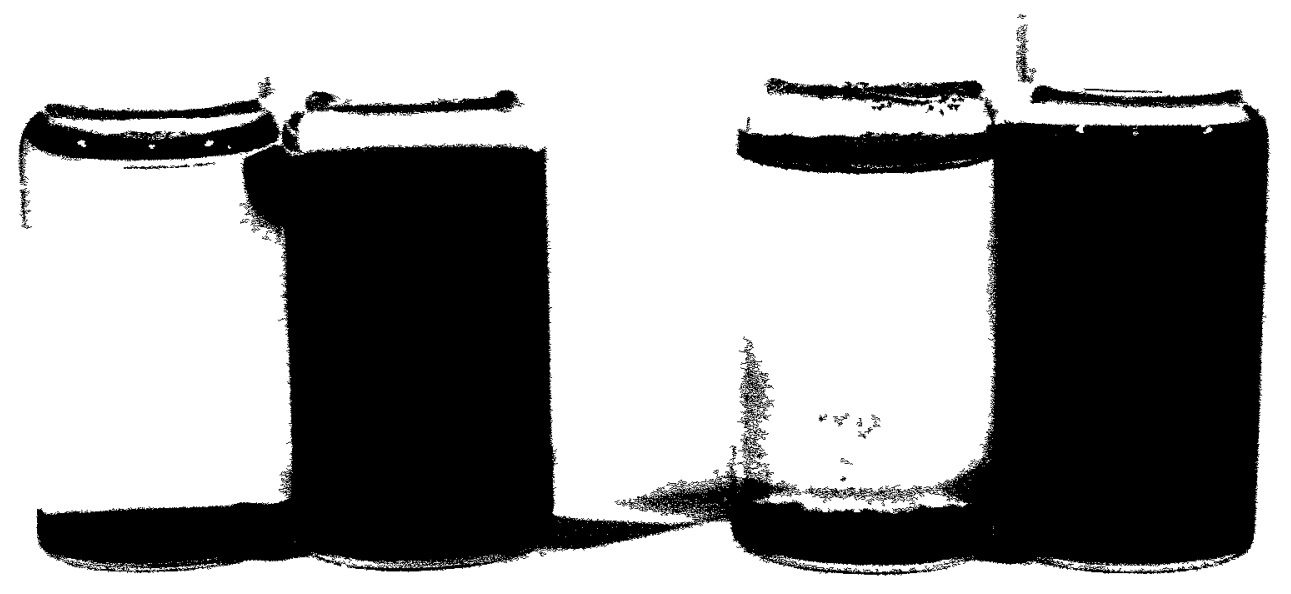

Figure 3-17 Comarison of aqueous suspensions of pristine and oxidized SWCNTs (left pair) and pristine and oxidized MWCNTs (right pair).

\subsubsection{Characterization of Polymer Membranes}

Scanning electron microscopy was also used to confirm the diameters of the pores in the membranes used to make the CNT envelopes. Membrana $\mathrm{GmbH}$, the manufacturer of the polypropylene membrane, indicated on the product website that the nominal pore size of this membrane was $0.2 \mu \mathrm{m}$. The SEM image of this membrane shown in Figure 3-18 confirms that the pores vary in diameter but the average pore size is likely around $0.2 \mu \mathrm{m}$. Similarly, Millipore, the manufacturer of the both kinds of polyvinylidene fluoride membranes, indicated on the website that the effective pore diameters were $0.1 \mu \mathrm{m}$ and $0.65 \mu \mathrm{m}$, respectively. The SEM image of the membrane with pore diameter $0.1 \mu \mathrm{m}$, shown in Figure 3-19, suggests that the pores were larger than $0.1 \mu \mathrm{m}$; however the pores 
observed may not be representative the average pore diameter. Conversely, the average pore diameter of the pores measured on the image of the other PVDF membrane, shown in Figure 3-20, indicates that the average pore size of this membrane is indeed close to $0.65 \mu \mathrm{m}$. All three SEM images of the membranes demonstrate that the pores in either membrane are not straight channels perpendicular to the surface of the membrane, but rather twisted varying pathways throughout the membrane.

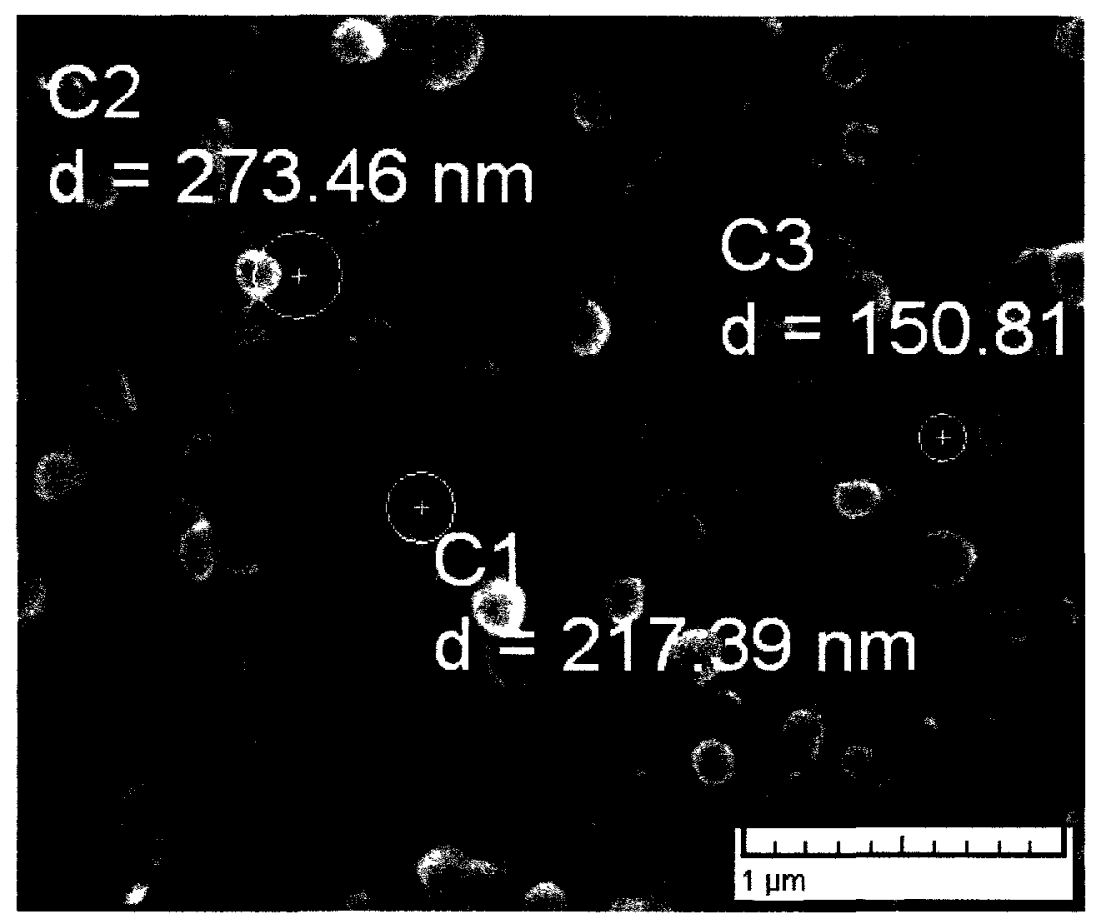

Figure 3-18 SEM image of the polypropylene membrane, with a pore diameter of $0.2 \mu \mathrm{m}$, as reported by the manufacturer, Membrana $\mathrm{GmbH}$. 


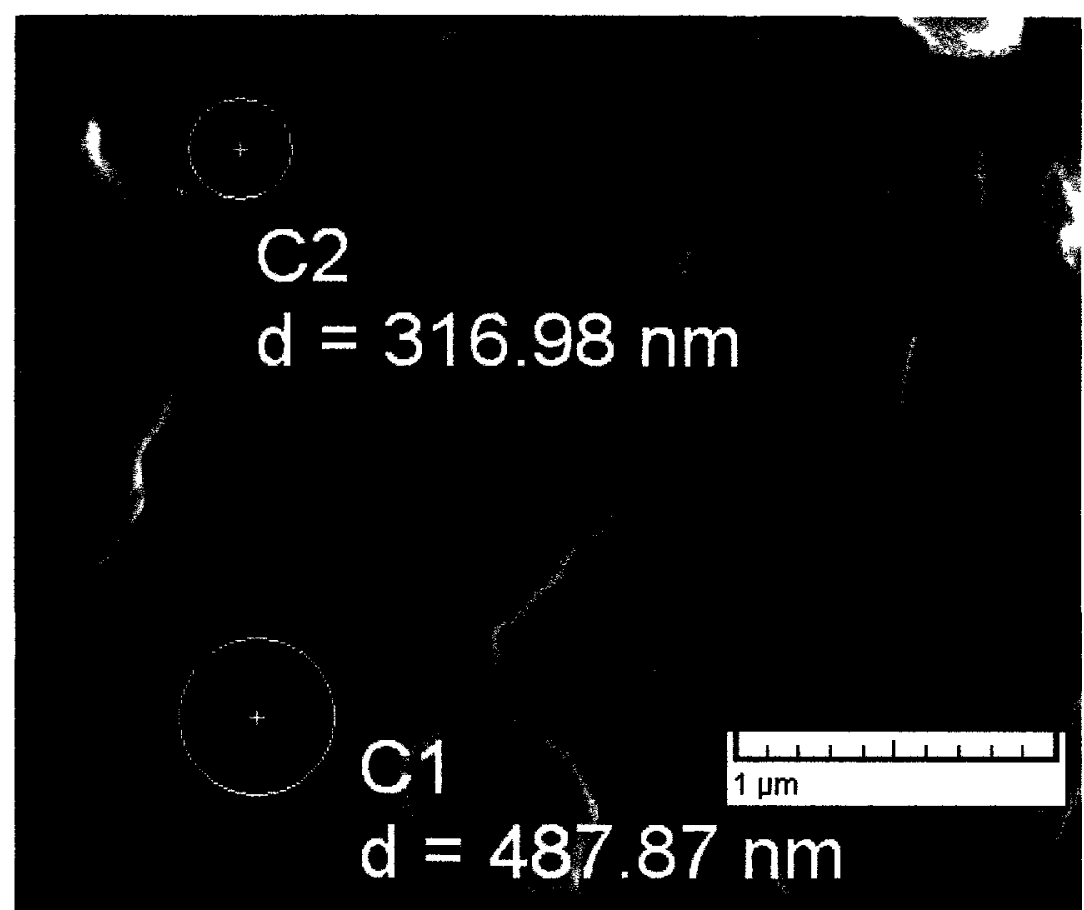

Figure 3-19 SEM image of the polyvinylidene fluoride membrane, with a pore diameter of $0.1 \mu \mathrm{m}$, as stated by the manufacturer, Millipore.

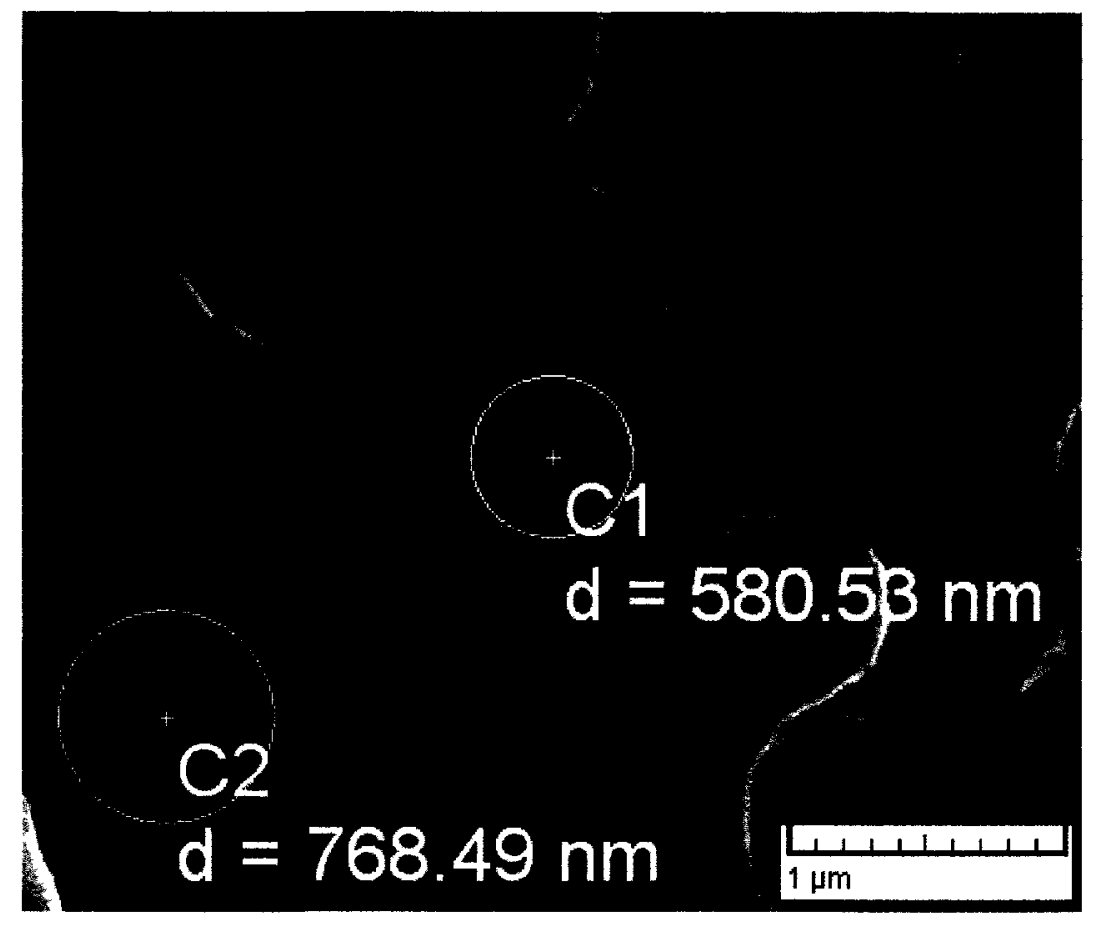

Figure 3-20 SEM image of the polyvinylidene fluoride membrane, with a pore diameter of $0.65 \mu \mathrm{m}$, as stated by the manufacturer, Millipore. 


\subsection{Carbon Nanotube Envelopes}

As previously mentioned, the discovery of carbon nanomaterials led to the development of a whole new class of SPE sorbents. Carbon nanotubes are an advantageous sorbent choice due to their high surface area. Futhermore, they possess the ability to $\pi$ - $\pi$ stack with aromatics which makes them an excellent choice for extraction of such contaminants. The extremely small dimensions of CNTs make them difficult to filter and so CNTs are most commonly packed in an empty SPE cartridge. Despite possessing a high surface to volume ratio, tens to hundreds of milligrams of tubes have been reported for used for SPE which is unnecessary and costly. The toxicological properties of CNTs are currently being studied and thus far it is unclear what risks are associated with carbon nanotube exposure. In the case of drinking water analysis, it is important to safeguard the extraction media from CNT contamination should they potentially cause adverse health effects. The aim of this project was to fabricate an SPE device, containing small quantities of CNTs, which could be used for BPA extraction from aqueous solution. The carbon nanotubes would be trapped within the device preventing CNT contamination to the aqueous extraction media and protecting the CNTs from any particulate matter in the sample matrix.

The idea to create a CNT envelope was based on the work of Basheer et al. [97] who reported the development of this $\mu$-SPE device. The rectangular $\mu$-SPE device was prepared by folding a piece of polypropylene membrane in half and then sealing two of the three open edges with heat. Then, MWCNTs were introduced using a glass funnel and Pasteur pipette. Last, the remaining unsealed edge was also heat sealed to secure the 
contents inside. Although this design worked well, it seemed tedious to seal the three open edges individually. If all edges could be sealed simultaneously, fabrication of the device would be more efficient and less time and energy would be required to make each seal. The easiest way to overcome this challenge would be to heat an object that when used to form a seal would encapsulate the CNTs between two pieces of membrane along every edge in a single use, such as a circular instrument. Consequently, the concept to create a CNT envelope was developed wherein CNTs would be sandwiched between two pieces of membrane and a heated circular tool would be used to stamp a seal on the top membrane around the carbon nanotubes underneath. A combination of heat and pressure was used to create the seal between the bottom and top membranes. It was hoped that the final product would model a commercial teabag, wherein porous paper is commonly used to contain loose tea. Like loose tea in a teabag, the carbon nanotubes would be protected by the membrane and would not be released into the water outside the membrane.

Initially, polypropylene was chosen as the encapsulating membrane to fabricate the envelope due to its low melting point. The $\mu$-SPE device fabricated by Basheer et al. [97], also made from polypropylene, was successful for extraction of acidic drugs [98] in wastewater and organophosphorous pesticides in sewage sludge samples [97]. Long multi-walled CNTs, with diameter $10 \mathrm{~nm}$, were chosen as the adsorbent in this work due to their high surface area and low cost compared to SWCNTs and DWCNTs. Initially, the CNTs were placed in between two pieces of PP membrane and a glass vial was heated with a heat gun and pressed against the top membrane to create a seal. Since the membranes were white and opaque, it was difficult to judge where to press the heated 
vial. As a result, sometimes the vial would be stamped directly over top of the CNTs which would prevent the sheets from sealing since they were not in direct contact. Also, CNTs are very fine and powdery, and when the top piece of membrane was placed over top of the small CNT pile; they tended to disperse between the membranes. Furthermore, the two pieces of membrane were not held in place with anything and thus could move easily if something brushed by them accidently.

After describing the intended design of the CNT envelope to the staff at the Science Technology Center at Carleton University, several stainless steel tools were created to aid in CNT envelope synthesis. The challenges that were initially experienced preparing the PP-CNT envelopes were overcome. The CNTs were collected in the middle of the exposed bottom PP sheet using the centering tool. Although they were no longer visible when the top PP sheet was placed overtop, the opening in the centering jig guided the sealer tool directly to the spot where the seal was supposed to be created. The centering jig and die block also held the membranes in place. Some static was still observed when the top PP sheet was placed on the CNTs through the centering jig, however with practice the static effects were minimized.

For reasons that will be discussed later, the membrane used to prepare the CNT device was changed to polyvinylidene fluoride. This membrane was supplied as $47 \mathrm{~mm}$ discs, unlike the polypropylene membrane which was supplied as a roll. A PVDF disc fit perfectly between the centering jig and die block and could be used as the bottom membrane as received. A second disc was too big in diameter to fit through the opening in the centering jig and needed to be cut for use as the top PVDF membrane. The top PP 
punch was used to measure the disc exactly to size, however the PVDF membrane was more durable than the PP membrane and was not easily cut with the punch. To avoid cutting every PVDF disc by hand, changes were made to the envelope synthesis. The centering tool was still used to center the CNTs on the bottom PVDF sheet, however the bottom PVDF sheet was placed on a glass filter that was part of a vacuum setup. To overcome the problems caused by static, 1-2 $\mathrm{mL}$ of methanol were passed through the centering tool to wet the CNTs and hold them in place. After, the bottom PVDF sheet was still placed on the die block as before and the centering jig was placed overtop, although the second $47 \mathrm{~mm}$ PVDF disc was held in place on the inside surface. The use of methanol greatly reduced the effects of static electricity between the CNTs and the membrane, however minor effects of static were observed when the top PVDF sheet was fitted over the bottom sheet using the centering jig. Refer to Figure 2-2 through Figure 216 in Sections 2.7 and 2.9 for photographic descriptions of CNT envelope synthesis with both types of membrane.

Although the techniques used to prepare PP-CNT and PVDF-CNT envelopes were unique, each method had advantages and disadvantages. Preparing the bottom and top PP sheets using the punches was time consuming compared to using the PVDF discs as received. Furthermore, polypropylene is a more fragile membrane compared to the PVDF membrane and it ripped easily, especially when the top and bottom PP sheets were being cut. When the CNTs were wet with methanol, for synthesis of PVDF-CNT envelopes, less static was observed than when they were just collected on the bottom PP sheet with the centering tool. Since the melting point of polypropylene is lower than that 
of polyvinylidene fluoride, it was significantly easier to create a seal to prepare a PPCNT envelope. The sealer tool needed to be heated to a higher temperature in order to melt the two PVDF membranes together upon contact, compared to the PP membranes. Despite the use of methanol to collect the CNTs, some CNTs still dispersed as a result of static electricity when the top PVDF sheet was placed overtop using the centering jig. Consequently, it was difficult to create a seal using both types of membranes if they were not in direct contact.

Both synthesis techniques were successful at preparing CNT envelopes. The carbon nanotubes inside were loose and could move around within the envelope. The seal between membranes generally remained intact, except when the envelope was subjected to ultrasonication. Interestingly, polypropylene and toluene have similar indexes of refraction and when the opaque membrane was immersed in toluene it turned clear. A photo of a PP-CNT envelope stored in toluene is shown Figure 3-21 to give a better idea of what the envelope looked like on the inside. The final device (made from either membrane) could be used for BPA removal from aqueous solution and could survive general wear and tear experienced during extraction. 

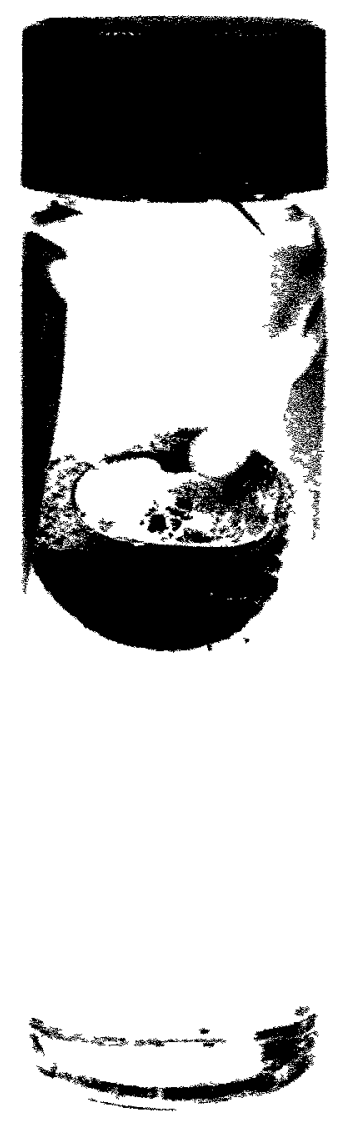

Figure 3-21 A PP-CNT envelope immersed in toluene to better illustrate what a CNT envelope looks like on the inside.

\subsection{Overview of BPA extraction using CNT envelopes}

BPA extraction from water occurs when the BPA has a greater affinity for the carbon nanotubes than the aqueous solution. In order for adsorption to occur, the bonds between BPA and water and water and the carbon nanotubes must be broken so that bonds between BPA and the carbon nanotubes can be formed. In the CNT envelope system, a concentration gradient exists between BPA in solution and BPA inside the envelope. As a result, BPA must first diffuse through the pores of the membrane before it can reach the surface of the carbon nanotubes. Since BPA adsorption to carbon nanotubes directly 
from aqueous solution was studied previously by Cai et al., BPA adsorption was only studied using CNT envelopes in the present work. Adsorption was also studied with envelopes that did not contain any carbon nanotubes to determine if BPA also adsorbed to the porous membrane.

BPA adsorption to CNT envelopes was examined using envelopes made with two different types of porous membrane. First, polypropylene, a hydrophobic membrane, was chosen for its low melting point and reported use by Basheer et al. to fabricate $\mu$-SPE devices. BPA recovery using the polypropylene CNT envelopes, or PP-CNT envelopes, was low $(\sim 10 \%)$. The surface tension of water on the polypropylene surface was likely too high to allow any water migration through the pores of the hydrophobic membrane. Similarly, Basheer et al. reported low recoveries of organophosphorous pesticides in sewage sludge samples when polypropylene was used to make the $\mu$-SPE devices. The authors indicated that conditioning the devices in organic solvents prior to extraction in sewage sludge significantly improved extraction. Thus, in the present work, several organic solvents were used to condition the PP-CNT envelopes prior to immersion in aqueous BPA solution. The non-polar solvents, hexane and toluene did not improve extraction efficiency at all, while the polar solvents, acetone and methanol, greatly enhanced BPA recovery. When BPA recovery using conditioned empty PP envelopes (without carbon nanotubes) was examined, at least $50 \%$ of BPA in the system was extracted by the envelopes conditioned in acetone or methanol. As a result, the carbon nanotubes could not be isolated as the only adsorption site in the system. The 
conditioning solvent introduced too many complications to the adsorption system and thus BPA extraction using the PP-CNT envelopes was not pursued any further.

To ensure the use of conditioning solvents could be avoided, a hydrophilic membrane with a relatively low melting point was sought after to fabricate the CNT envelopes. Polyvinylidene fluoride (PVDF) was chosen and adsorption was examined when the PVDF-CNT envelopes were placed directly in aqueous BPA solution for extraction. After determining an ideal method of suspending the envelopes in solution, BPA recovery using the PVDF-CNT envelopes was determined to be about $50 \%$, which was twice as much as extracted by the empty PVDF envelopes. Since the ratio of aqueous BPA solution to CNT mass was so large, BPA adsorption using PVDF-CNT envelopes was more of an equilibrium extraction rather than an exhaustive extraction. Thus, BPA extraction using PVDF-CNT envelopes was examined over a range of temperatures, $\mathrm{pH}$ 's, concentrations and sample volumes. The time required for equilibrium of BPA adsorption to the envelopes was determined. Adsorption was also explored when the PVDF-CNT envelopes were filled with CNTs of different diameters and lengths.

\subsection{Initial BPA Extraction Using PP-CNT Envelopes}

After successful production of the PP-CNT envelopes, an initial extraction was performed to determine if the envelope could actually extract BPA from aqueous solution. A PP-CNT envelope was compared against a blank PP envelope (with no CNTs) to determine how much BPA adsorbed to the carbon nanotubes and how much adsorbed to the polypropylene membrane, if any. A fairly concentrated solution of BPA in water $(600 \mathrm{ng} / \mathrm{mL})$ was prepared to use during the extraction so that if only a small 
portion of the BPA adsorbed to the surface of the carbon nanotubes or membrane it could still be detected by the detector. The PP-CNT envelopes float in water so the extraction was performed in a glass vial that could just hold $40 \mathrm{~mL}$ of solution and could be sealed with a cap. Since the vial was filled to the top with solution, and the PP-CNT envelope could not fit through the neck of the vial, it was completely immersed in water. Although the work of Basheer et al. [97] indicated adsorption of organophosphorous pesticides from a sewage sludge sample reached equilibrium in $\sim 60$ minutes using the $\mu$ SPE device, an adsorption time of 24 hours was chosen for this initial extraction to ensure adsorption occurred, since BPA extraction from aqueous solution is similar but not identical to extractions performed using a $\mu$-SPE device. The PP-CNT and blank PP envelopes were allowed to tumble freely in aqueous solution for 24 hours during adsorption. Similarly, a desorption time of 24 hours was chosen to ensure complete desorption of BPA from the envelopes, despite the studies published by Basheer et al. [97] which reported significantly shorter desorption times. Although the experimentally measured solubility of BPA in methanol was determined to be approximately 655 $\mathrm{mg} / \mathrm{mL}$, the PP-CNT envelope was desorbed in $40 \mathrm{~mL}$ of methanol to ensure complete desorption was also achieved. Methanol was chosen as the desorption solvent since it was compatible with the mobile phase, it readily dissolves BPA and it has been documented in literature for the successful desorption of BPA from carbon nanotubes. The remaining water and methanol extracts were analyzed by HPLC to determine how much BPA was left in water after adsorption and the amount of BPA extracted using the PP-CNT envelope, which was measured by the amount of BPA desorbed from the envelope into methanol. 
Using BPA in methanol standards, the concentrations of BPA left in water and BPA extracted could be calculated. These values were multiplied by the volume of water or methanol used to determine the mass of BPA left in water or mass of BPA extracted, respectively. The original BPA in water standard used for extraction was also analyzed by HPLC to determine its true concentration. This value was multiplied by the volume of water used for extraction (in this case $40 \mathrm{~mL}$ ) to determine the mass of BPA in the water solution prior to extraction. Last, the \% BPA recovered, either left in water or extracted by the PP-CNT envelope, could be calculated by dividing the mass of BPA left in water or extracted by the PP-CNT envelope by the mass of BPA in the original aqueous solution and multiplying by $100 \%$, as shown below.

\% BPA Recovered $=$ Mass $\mathrm{BPA}_{(\mathrm{H} 2 \mathrm{O} \text { or } \mathrm{MeOH})} /$ Mass BPA in original BPA in $\mathrm{H}_{2} \mathrm{O}$ solution *100\%

Note: from here on, almost all figures will have a y-axis label of \% BPA Recovered, which indicates a comparison of the mass of BPA in either water or methanol, divided by the mass of BPA in the original aqueous solution.

The \% BPA left in water and \% BPA extracted by the blank and PP-CNT envelopes in this initial extraction are shown in Figure 3-22. 


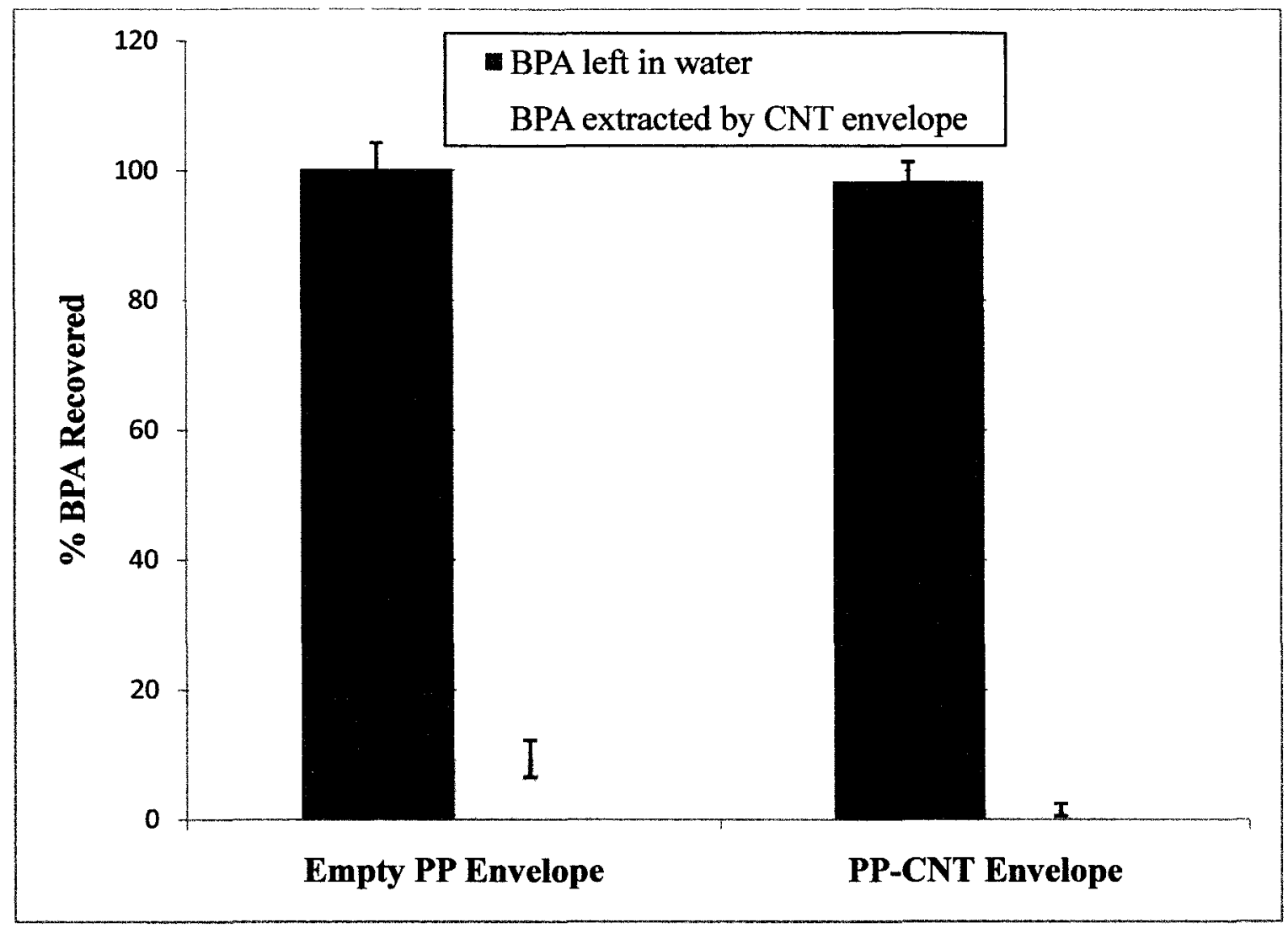

Figure 3-22 Comparison of BPA recovery between an empty PP envelope and PP--CNT envelope when the envelopes were placed in water directly for BPA extraction.

It can be seen that neither envelope was effective for significant BPA removal since essentially all BPA (at least $90 \%$ ) remained in aqueous solution after extraction. For the case of the blank PP envelope, this result is ideal. The purpose of the empty envelope was to determine if the membrane itself could be participating in the extraction, rather than just act as a barrier between the CNTs and aqueous solution. The possibility existed that some BPA may adsorb to the polypropylene surface. Furthermore, BPA is a largely organic molecule, despite its $-\mathrm{OH}$ groups and it was also possible that BPA may be soluble in the PP sheet, since it has been reported that other polymers are capable of dissolving organic analytes [101]. However, the results displayed in Figure 3-22 indicate 
that BPA will not significantly adsorb to nor be absorbed by the polypropylene sheet. Although BPA is soluble in many organic solvents that range in polarity, it is not very soluble in extremely non-polar solvents such as hexane, and the PP membrane may be too non-polar to sorb any BPA. The fact the PP-CNT envelope was not successful for BPA extraction can be explained by the hydrophobicity of the PP membrane. Although the pores of the membrane were significantly larger in diameter than the length of a BPA molecule and could definitely accommodate transport of BPA molecules, it is likely that no water entered the CNT envelope through the pores since the membrane is so hydrophobic. The surface tension of water on the polypropylene membrane was likely too great to allow any water migration through the envelope. Instead, a pocket of air could form in the membrane pore since the water molecules would repel the membrane as much as possible, as depicted in Figure 3-23. Consequently, water could not wet the envelope and no BPA molecules would reach the carbon nanotubes, preventing any adsorption. 


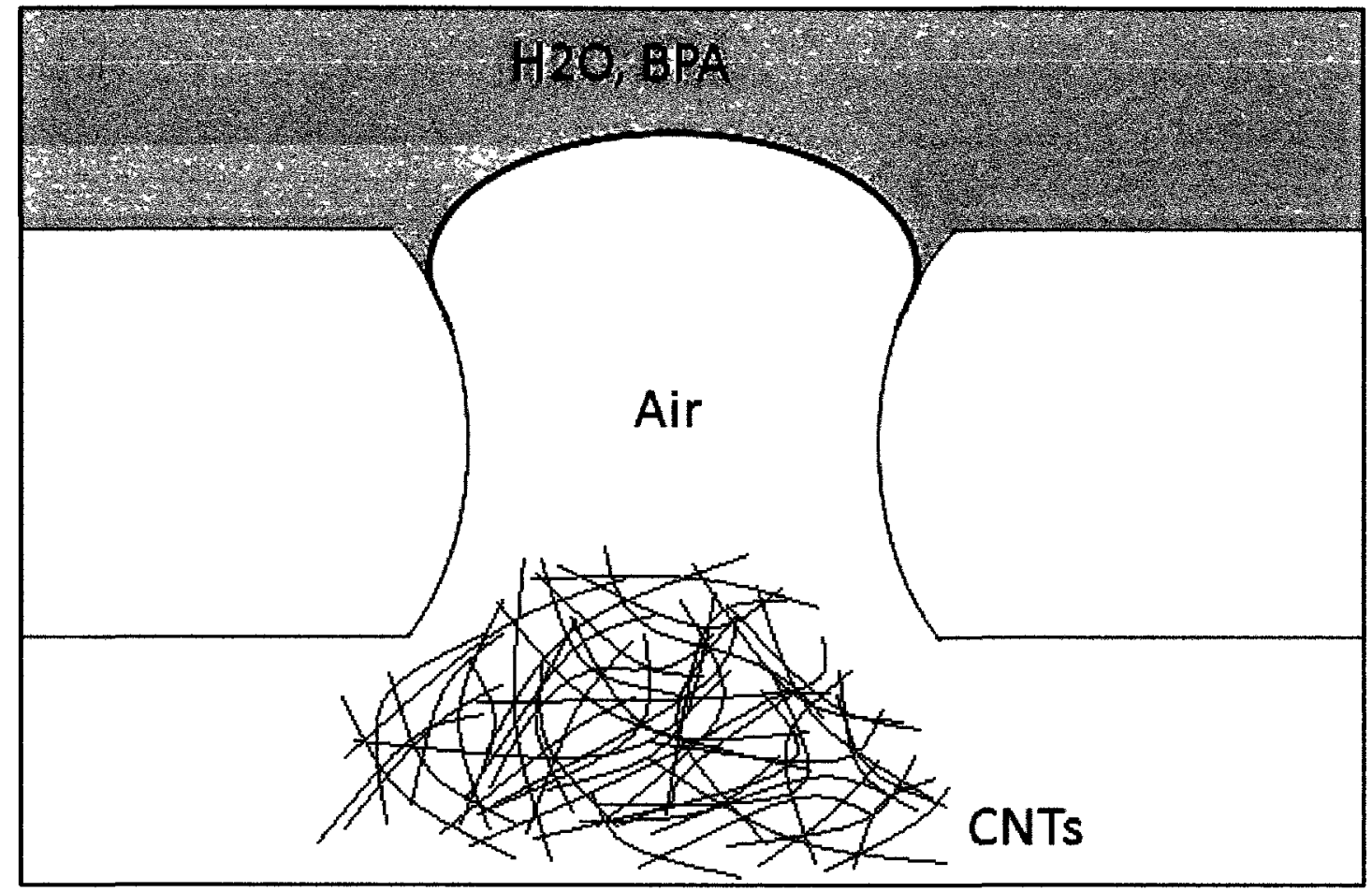

Figure 3-23 A schematic of possible interactions among water, BPA, CNTs and the polypropylene membrane during extraction. Repulsion between water and the hydrophobic membrane likely created a space in the pores of the membrane preventing water diffusion through the membrane and thus preventing BPA adsorption on the CNTs.

\subsection{PP-CNT Envelope Conditioning}

\subsubsection{Dipping in Organic Solvents}

Basheer et al. [97] reported low wettability when the $\mu$-SPE device was directly exposed to sewage sludge samples to extract organophosphorous pesticides. Consequently, low extraction efficiencies were observed. When the wettability of the $\mu$-SPE device was enhanced by conditioning the device in organic solvents prior to extraction, extraction efficiency increased significantly. Pesticide recovery was dependant on the solvent used. The organic solvent was said to be trapped in the pores of the membrane and act as a carrier to improve extraction recovery. To facilitate BPA extraction to PP-CNT 
envelopes, the use of an organic conditioning solvent was pursued to possibly promote adsorption to the CNTs.

To condition the CNT envelopes, they were immersed in the given solvent for $1 \mathrm{~min}$ and then subjected to sonication in water for $2 \mathrm{~min}$. Acetone and methanol were chosen initially since BPA is so soluble in both of these solvents, and they are both miscible with water. Two conditioning methods were explored. First a PP-CNT envelope was dipped in either acetone or methanol for a couple of seconds and was then transferred directly to the aqueous BPA solution for extraction. The second method involved soaking the PPCNT envelope in either acetone or methanol overnight, until use for extraction. When the envelope was dipped in the solvent, it appeared to inflate with solvent immediately after it was immersed. When the PP-CNT envelope was stored in the solvent overnight it did not appear to be any more inflated. This may indicate that only a few seconds of contact are necessary to condition the envelope and dipping in the organic solvent may be sufficient. Basheer et al. [97] indicated that extraction performed with an empty PP $\mu$ SPE device was not successful at extracting any pesticides. However, since no BPA extraction occurred using the blank PP and PP-CNT envelopes in the initial extraction, it was still unclear if the polypropylene membrane could act as a sorbent. Therefore, extraction using a conditioned blank PP envelope was also carried out simultaneously with the extraction of the PP-CNT envelope conditioned with either acetone or methanol.

Figure 3-24 and Figure 3-25 depict the BPA recovery when the envelopes were conditioned with acetone. It can be seen that the "acetone-dipped" PP-CNT envelope was successful at removing BPA from aqueous solution since more than $90 \%$ of BPA 
present in the system was extracted using the SPE device. Surprisingly, the blank PP envelope extracted just more than $50 \%$ of BPA as well. Similar results were obtained for extraction using envelopes soaked in acetone overnight. Again, the PP-CNT envelope showed a high extraction capability, but the empty envelope also removed a significant portion of BPA from aqueous solution.

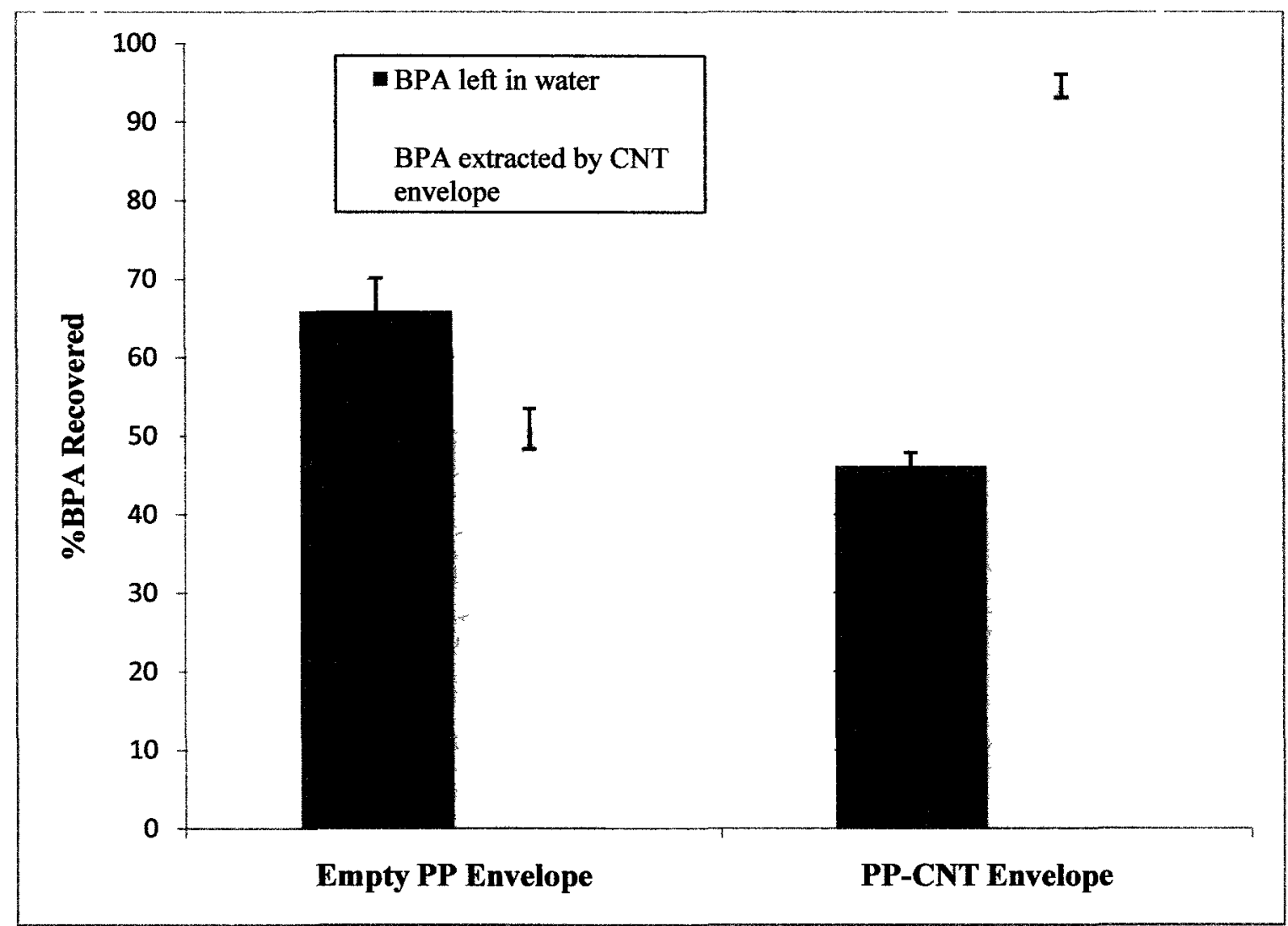

Figure 3-24 BPA recoveries when the PP devices (empty and filled) were conditioned by dipping in acetone prior to use. 


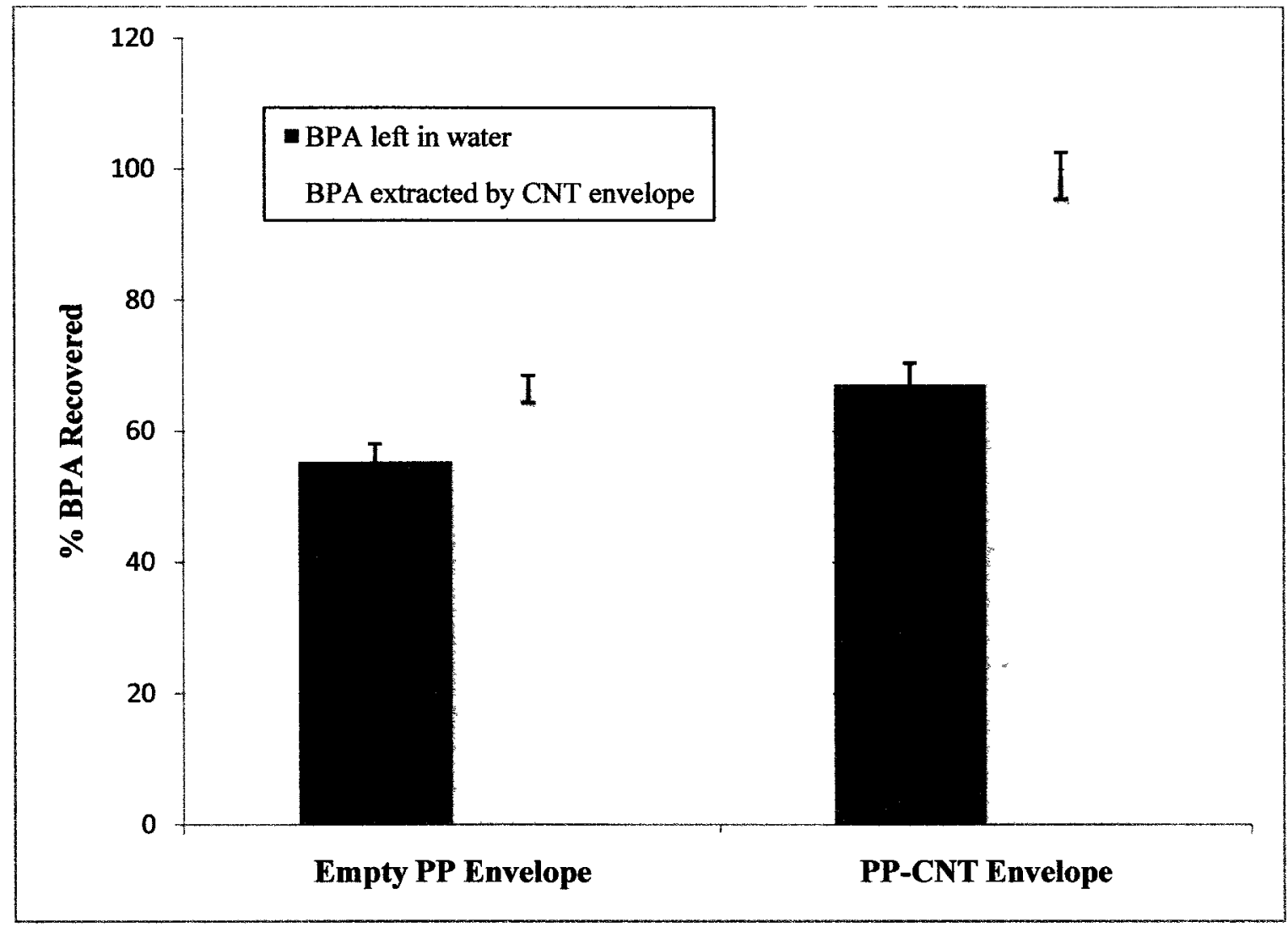

Figure 3-25 BPA recoveries when the PP devices (empty and filled) were conditioned by soaking in acetone overnight prior to use.

Similarly, Figure 3-26 and Figure 3-27 depict the BPA recovery when the empty and filled PP envelopes were dipped and soaked in methanol overnight, respectively. Again, it is evident from these figures that contacting the polypropylene sheet with methanol facilitates transport of BPA through the membrane. The extraction efficiencies were high for both methods of conditioned PP-CNT envelopes. Likewise with acetone, the empty envelopes were also capable of extracting BPA from aqueous solution. When dipped in methanol, the amount of BPA removed using the empty and filled PP envelopes was indistinguishable. In other words, the presence of the solid-phase, carbon nanotubes, had 
no effect on extraction. When the envelopes were soaked in methanol overnight, the PPCNT envelope removed a slightly greater amount of BPA, as seen in the case of acetone.

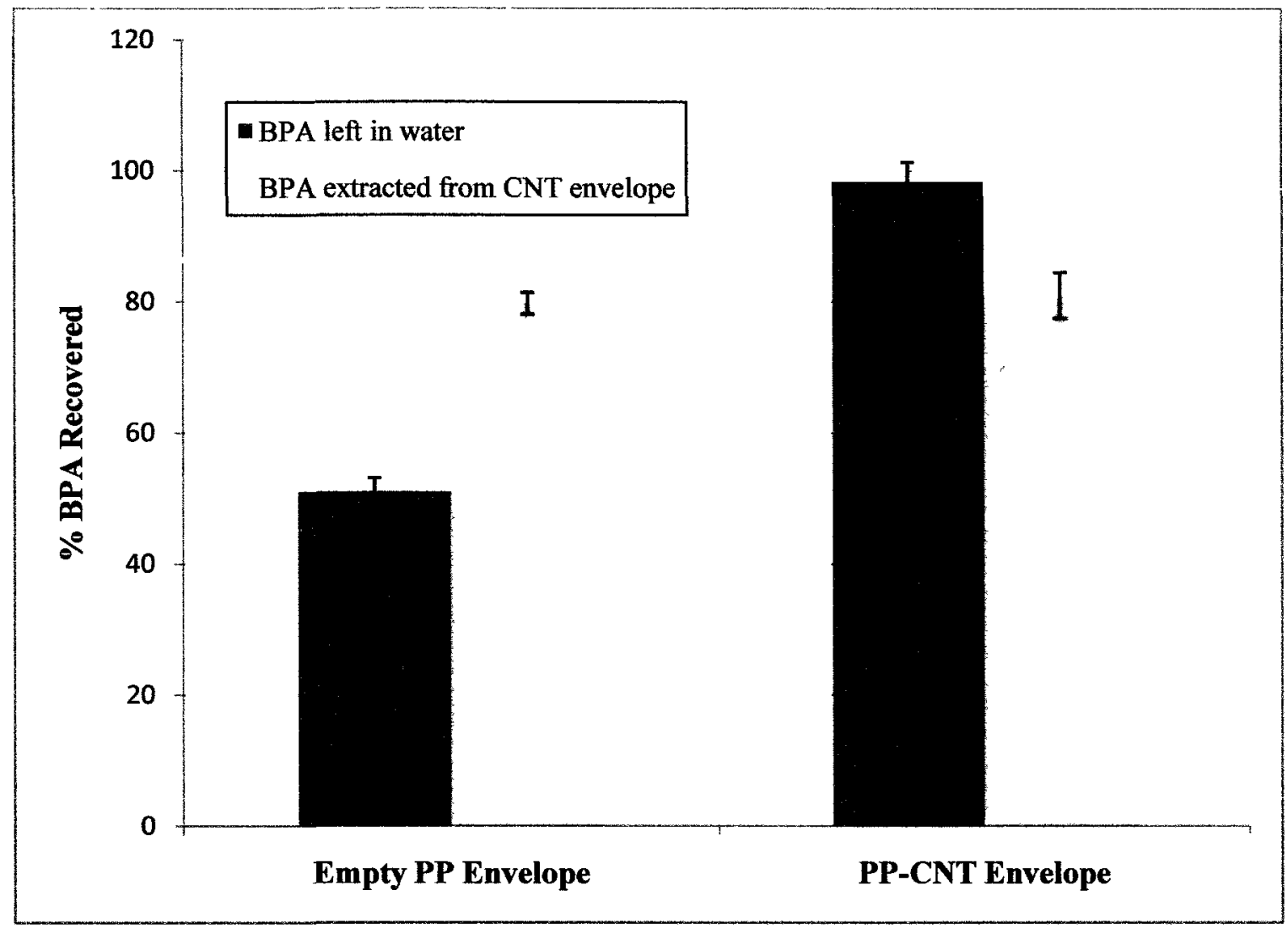

Figure 3-26 BPA recoveries when the PP devices (empty and filled) were conditioned by dipping in methanol prior to use. 


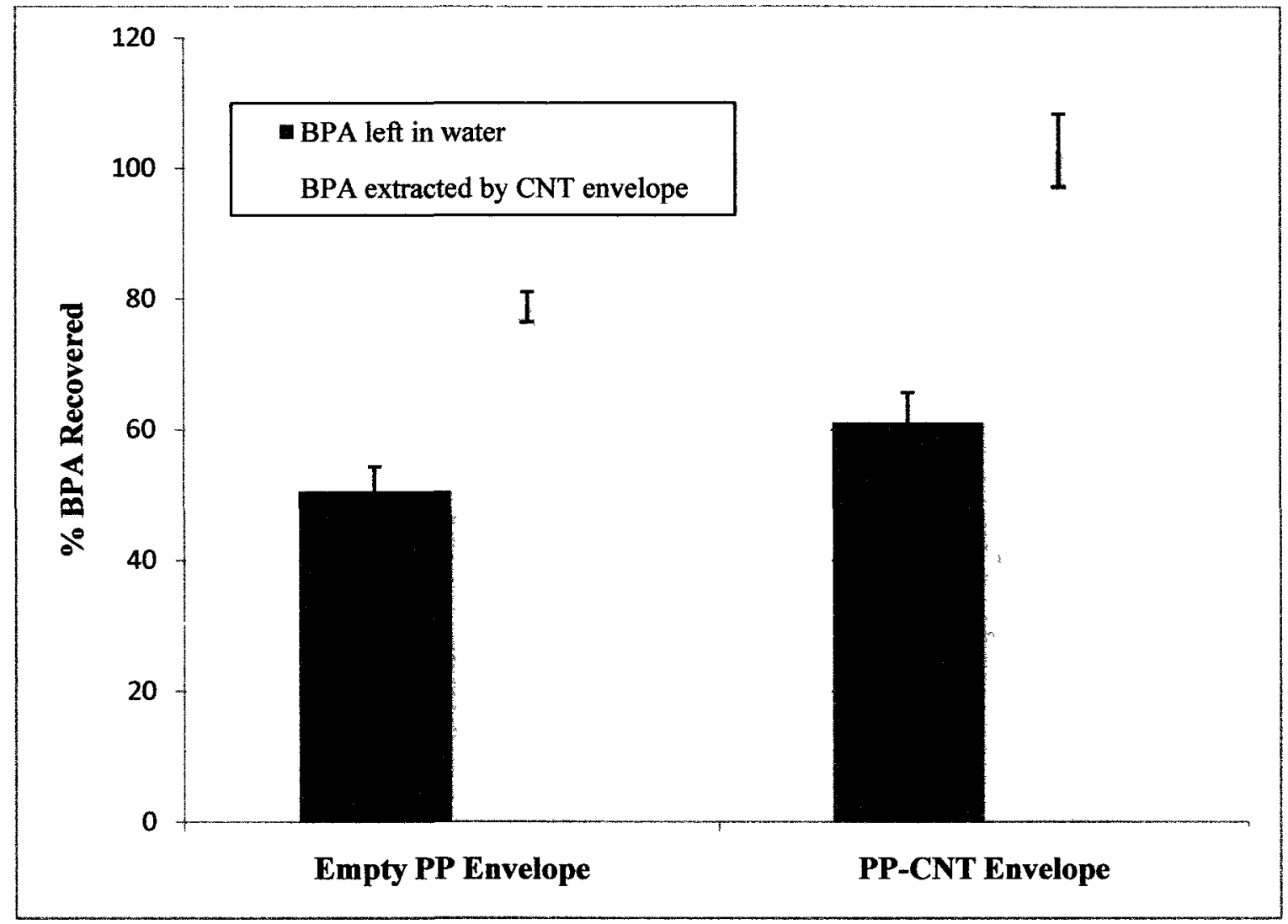

Figure 3-27 BPA recoveries when the PP devices (empty and filled) were conditioned by soaking in methanol overnight prior to use.

These results indicate that the solvent present in the envelope after conditioning must be participating in extraction. BPA is extremely soluble in both acetone and methanol and it is conceivable that a significant amount of BPA present in the system would partition to the organic solvent phase. When the four plots examining envelope conditioning between empty and filled devices are compared, there is a difference in the amount of BPA extracted when CNTs are present, with the exception of the envelopes dipped in methanol. Although the increase in \% BPA recovered between empty and filled CNT envelopes is not as substantial as the amount extracted by the empty envelopes themselves, the difference is greater by at least $20 \%$ in all cases. Thus the carbon 
nanotubes also have an impact on adsorption. Water is miscible with both acetone and methanol and so these solvents were chosen for envelope conditioning, in hope the presence of the solvent would aid water transport through the membrane. However, if the conditioning solvent was less polar, the amount of BPA extracted by the solvent may be considerably less but water flow through the envelope may still be possible to allow BPA adsorption to CNTs. Consequently, the empty PP envelope may not sorb as much BPA.

To address this point, BPA extraction from water was measured when the PP-CNT envelope was dipped in dichloromethane. Since no significant improvements to extraction efficiency were observed if the envelope was soaked in methanol or acetone overnight, the PP-CNT envelopes were only dipped in this less polar solvent for a few seconds. Figure 3-28 indicates BPA extraction when the PP-CNT envelope was dipped in dichloromethane prior to extraction. Overall, the amount of BPA extracted by either the empty or CNT-filled envelope was markedly less when the envelopes were conditioned with dichloromethane. Although BPA is still moderately soluble in dichloromethane, its solubility is significantly lower compared to acetone or methanol. This is reflected in the amount of BPA recovered between envelopes conditioned in acetone and methanol and with dichloromethane. Also, the amount of BPA extracted by the empty envelope was much lower than when the empty envelope was conditioned with the more polar solvents. However, the ratio of $\%$ BPA extracted between the empty and filled PP envelopes remained the same. 


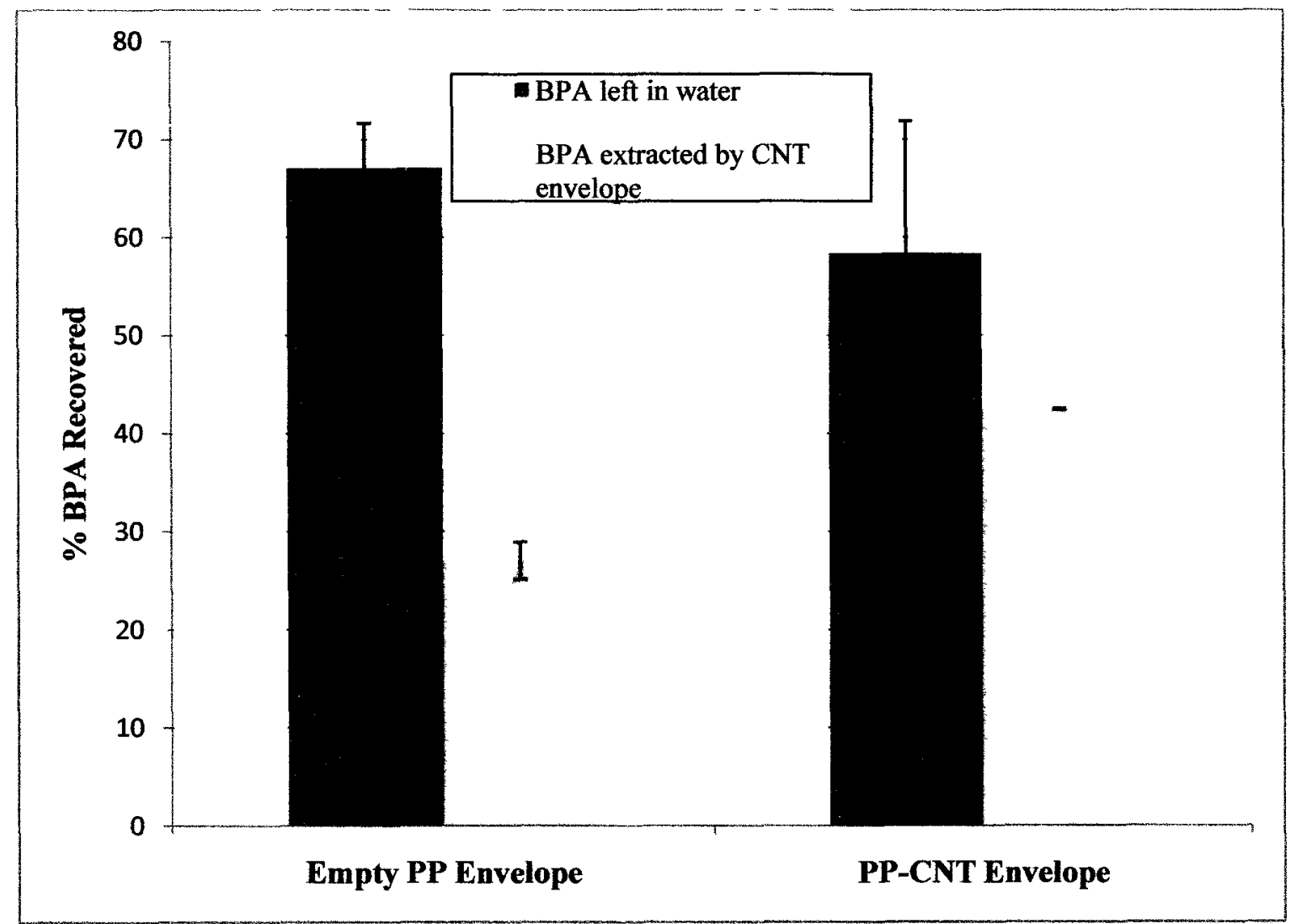

Figure 3-28 BPA recoveries when the PP devices (empty and filled) were conditioned by dipping in dichloromethane prior to use.

Although extraction efficiency increased in the presence of CNTs in almost all conditioning experiments, the empty PP envelope still extracted BPA and when conditioned in acetone and methanol, was actually found to be a moderately effective SPE device. It was unclear exactly what interactions were happening at the membranewater interface. Water is miscible with acetone and methanol and when used to condition the PP-CNT envelope the distinction between water and solvent phases was blurred. As was the case for methanol and acetone, water transport into the membrane and solvent transport out of the membrane may both have occurred when the PVDF-CNT envelope was dipped in dichloromethane. In order to better understand the adsorption process, the 
CNT device was also conditioned with relatively polar water-immiscible solvent, dichloromethane. Conditioning the PP-CNT envelope with dichloromethane was less effective than acetone or methanol, however BPA was still extracted. To examine the extraction capability of the envelope over the spectrum of solvent polarity, the device was also conditioned with toluene and hexane. BPA is significantly less soluble in non-polar solvents, particularly hexane, and thus BPA partitioning to the organic phase was not expected to be significant.

The results from dipping the PP-CNT envelopes in toluene and hexane (not shown) were notably different from the recoveries observed when the envelope was dipped in more polar solvents. CNT devices dipped in either solvent were not successful at removing any BPA from aqueous solution. These results are not surprising. BPA solubility in toluene and hexane is several orders of magnitude lower than acetone and methanol. As the polarity of the conditioning solvent decreased from acetone/ methanol to dichloromethane, the amount of BPA recovered also decreased considerably. Therefore, a further decrease in polarity from dichloromethane to toluene and hexane would also likely follow this trend. Furthermore, water is immiscible with both of these solvents. In LLE techniques, significant agitation is required to partition analytes from aqueous solution to a water-immiscible organic phase in a reasonable amount of time. Here, BPA partitioning from water to the organic solvent would occur at a much slower rate since vigorous shaking methods were not employed. As stated in Table 3-1, the experimental solubility of BPA in deionized water at room temperature was determined to be $530 \mathrm{mg} / \mathrm{L}$ which is higher than reported literature values [20]. The experimental solubility of BPA 
in toluene and hexane was determined to be $3.98 \times 10^{3} \mathrm{mg} / \mathrm{L}$ and $60.5 \mathrm{mg} / \mathrm{L}$, respectively. Since BPA is more soluble in water than hexane, partitioning to the less soluble phase would be unlikely. When the solubility of BPA in water is compared to the solubility in toluene however, the solubility of BPA in water is approximately 8 times lower and partitioning into toluene would be expected. Slow partitioning rates may likely explain the absence of adsorption when toluene was used to condition the envelope.

While envelope conditioning in acetone and methanol was successful for removing BPA from PP-CNT envelopes, adsorption could not be isolated to just the envelope containing CNTs. As a result, alternative conditioning methods were explored to see if BPA extraction could be limited to the device filled with carbon nanotubes. The use of sonication was explored to condition the $\mu$-SPE device. Basheer et. al. indicated that the $\mu$-SPE device was cleaned by ultrasonication in acetone for 10 minutes before storing acetone until use. Thus, a blank PP envelope and PP-CNT envelope were sonicated in acetone for 30 minutes to condition the membrane in hope that the envelopes might not inflate with so much solvent. Figure 3-29 shows the effect of the use of sonication on extraction. Although the amount of BPA extracted increased with the PP-CNT envelope compared to the empty envelope, the blank envelope was still responsible for a significant amount of BPA extraction as seen before. Moreover, the PP-CNT could not withstand the harsh sonication conditions very well and the envelope seal easily burst open. In order to overcome this challenge, the membrane needed to be wet without allowing the organic solvent to fill the envelope completely. Thus, it was necessary to determine how much solvent was filling the envelope. 


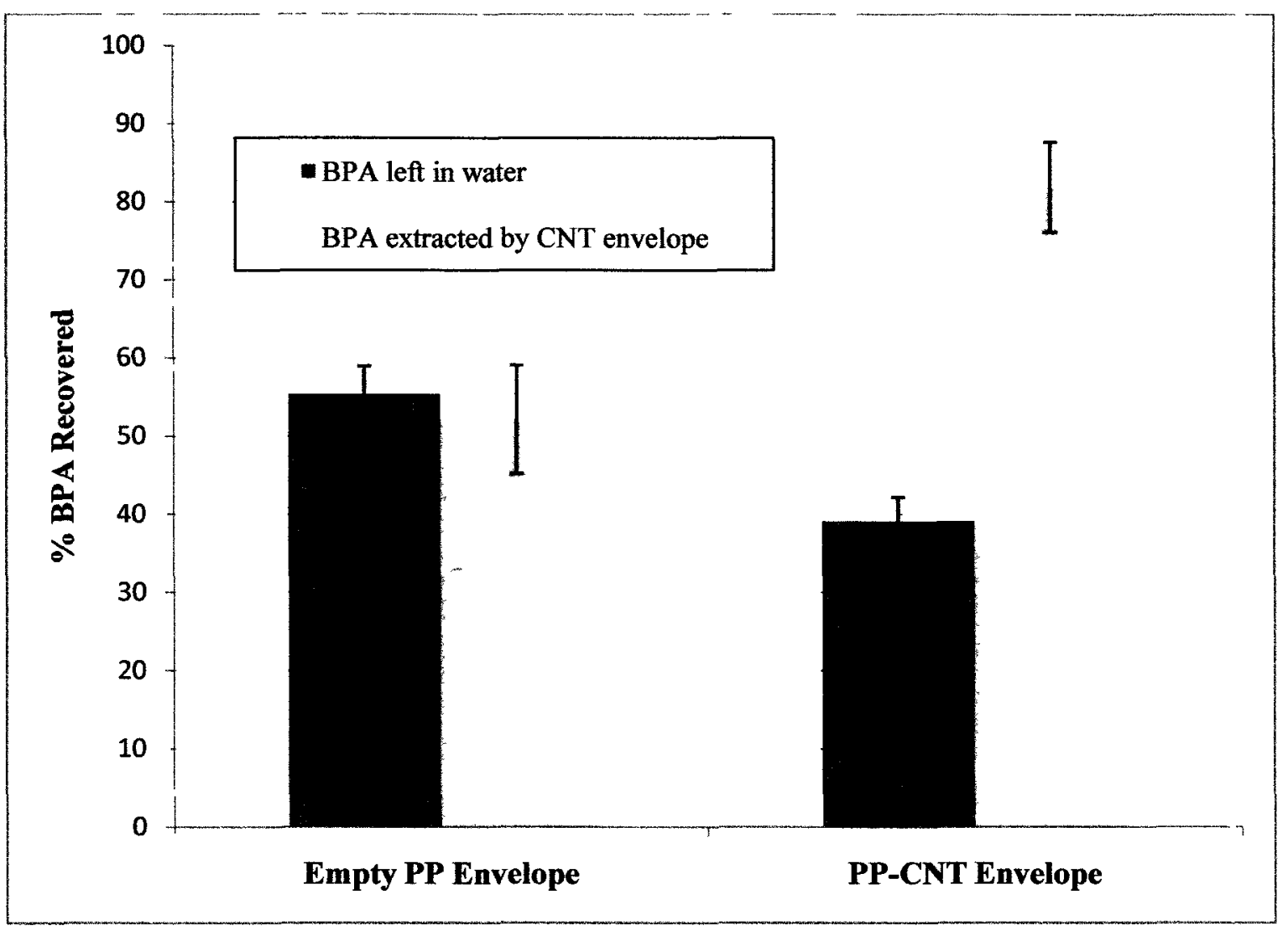

Figure 3-29 BPA recoveries when the PP devices (empty and filled) were conditioned by sonication in acetone prior to use.

As previously mentioned, there was no statistical difference in the amount of BPA extracted between envelopes dipped or soaked overnight in solvent. However, the length of time the envelope needed to be immersed in solvent to fill completely was not known. To calculate the amount of time required for a PP-CNT envelope to completely fill with solvent, the device was dipped in solvent for a period of time and then weighed immediately after. Once all solvent evaporated from the envelope, it was dipped in the same solvent again for a longer period of time. This procedure was repeated until the envelope mass reached equilibrium. Three solvents, hexane, acetone and methanol, were used to model this curve and were used to compare an empty PP envelope to a PP-CNT 
envelope. These solvents are all quite volatile so care was taken to weigh the envelope immediately after removing it from the organic solvent; however, discrepancies between data points indicate the rapid evaporation of solvent from the envelope. The results from all three solvents are shown in Figure 3-30. It can be seen from this plot that the given solvent saturated the envelope within 30 seconds of contact. Also, although different envelopes were used to depict each conditioning solvent, but it was noted in Figure 3-30 that the difference between the original mass of the PP-CNT envelopes and empty PP envelopes was approximately $6 \mathrm{mg}$ for each solvent, the mass of carbon nanotubes used to fill each envelope (exact masses are not shown).

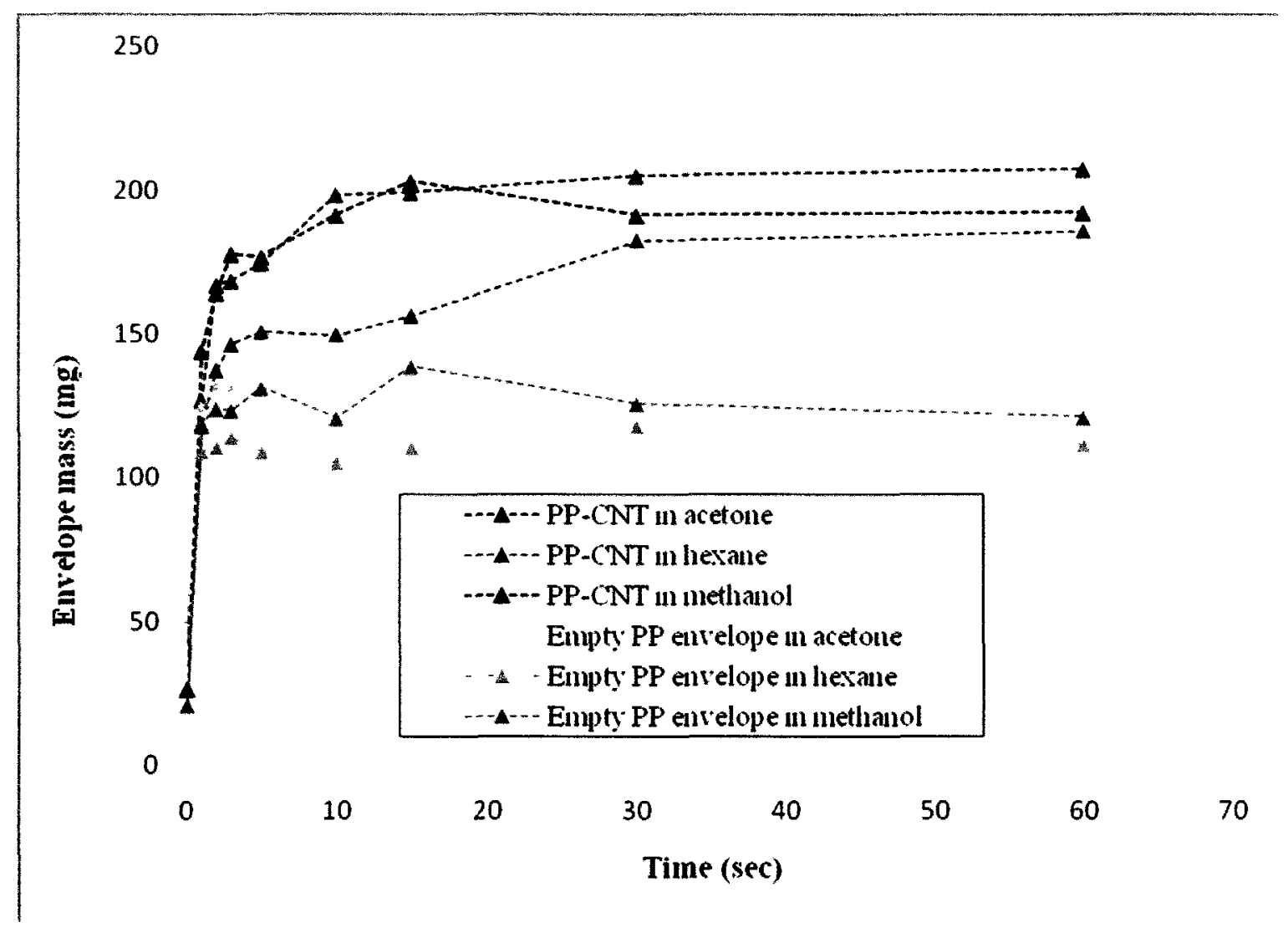

Figure 3-30 Time required for empty and filled PP envelopes to completely fill with solvent during condtitioning. 
To determine what volume fills the envelope when it is saturated with solvent, the mass of solvent needed to fill the envelope can be determined by finding the difference between the mass of the envelope before conditioning and after the envelope reached saturation which can then be multiplied by the density of the solvent. Table 3-3 below indicates the volume required to fill each envelope. The data in this table indicate that an empty PP envelope was saturated with at least $100 \mu \mathrm{L}$ of solvent upon conditioning, while PP-CNT envelopes could hold at least $200 \mu \mathrm{L}$. When the envelopes were dipped in solvent, they were only immersed in the solvent for a couple of seconds. The short amount of time the envelopes were in contact with the solvent may have been long enough to fill the envelope, which was confirmed by the visual inflation of the envelope.

Table 3-3 Volume of solvent needed to fill a PP-CNT envelope with acetone, hexane or methanol

\begin{tabular}{|c|c|c|c|c|c|c|}
\hline & $\begin{array}{c}\text { Empty } \\
\text { PP } \\
\text { envelope- } \\
\text { acetone }\end{array}$ & $\begin{array}{c}\text { PP-CNT } \\
\text { envelope- } \\
\text { acetone }\end{array}$ & $\begin{array}{c}\text { Empty } \\
\text { PP } \\
\text { envelope- } \\
\text { hexane }\end{array}$ & $\begin{array}{c}\text { PP-CNT } \\
\text { envelope- } \\
\text { hexane }\end{array}$ & $\begin{array}{c}\text { Empty } \\
\text { PP } \\
\text { envelope- } \\
\text { methanol }\end{array}$ & $\begin{array}{c}\text { PP-CNT } \\
\text { envelope- } \\
\text { methanol }\end{array}$ \\
\hline $\begin{array}{c}\text { Volume of } \\
\text { solvent } \\
\text { required to } \\
\text { fill envelope } \\
(\mu \mathrm{L})\end{array}$ & 0.119 & 0.225 & 0.138 & 0.241 & 0.132 & 0.212 \\
\hline
\end{tabular}

One last attempt was made to wet the PP-CNT envelope without allowing the envelope to fill with solvent. This time, the envelope was rolled or curled tightly and then dipped in either acetone or methanol for a couple of seconds to condition the membrane. It was hoped that the envelope would not fill with solvent, but just be wet enough to facilitate water diffusion. Since the envelope was wound so tightly, it did not appear to inflate 
with as much solvent as before. Again, both a blank CNT envelope and filled PP-CNT envelope were explored to determine how much BPA could be extracted by the blank envelope. Figure 3-31 and Figure 3-32 illustrate the effect of curling the envelope prior to conditioning in acetone and methanol, respectively. The amount of BPA recovered by the PP-CNT envelope in either case was relative to the amount recovered when the envelope was dipped in the same solvent without rolling. Both figures show that the presence of CNTs increased extraction efficiency compared to the blank envelopes. The result was more significant when methanol was used; the extraction capability of the CNT-filled envelope was almost $30 \%$ greater than that of the empty envelope. However, introduction of organic solvents into the extraction system still had a greater impact on extraction compared to the carbon nanotubes. Regardless of the conditioning solvent used, the blank envelope was capable of extracting $65 \%$ of BPA from aqueous solution, a much greater impact than the presence of CNTs. 


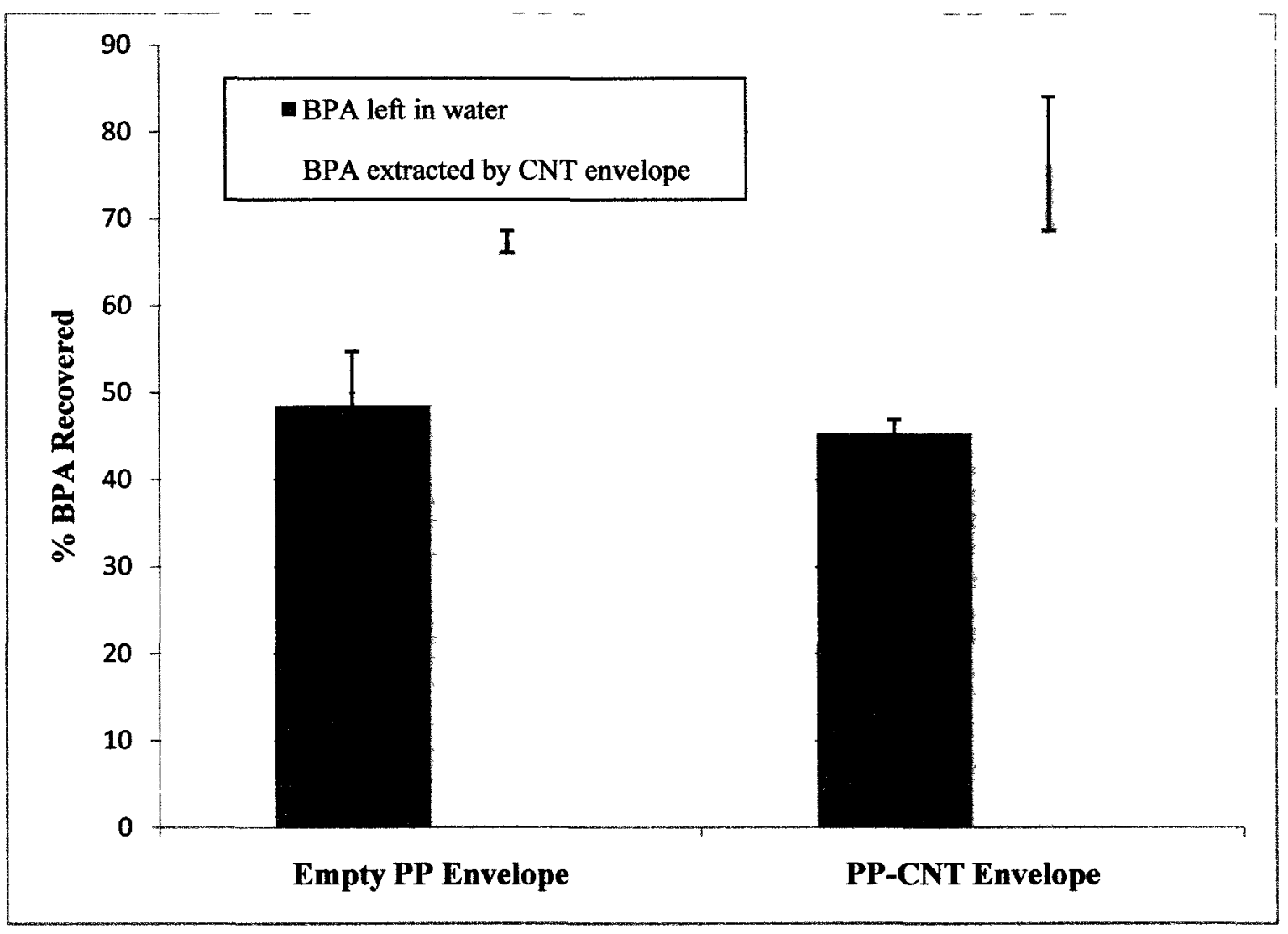

Figure 3-31 BPA recoveries when the rolled PP devices (empty and filled) were conditioned by dipping in acetone prior to use. 


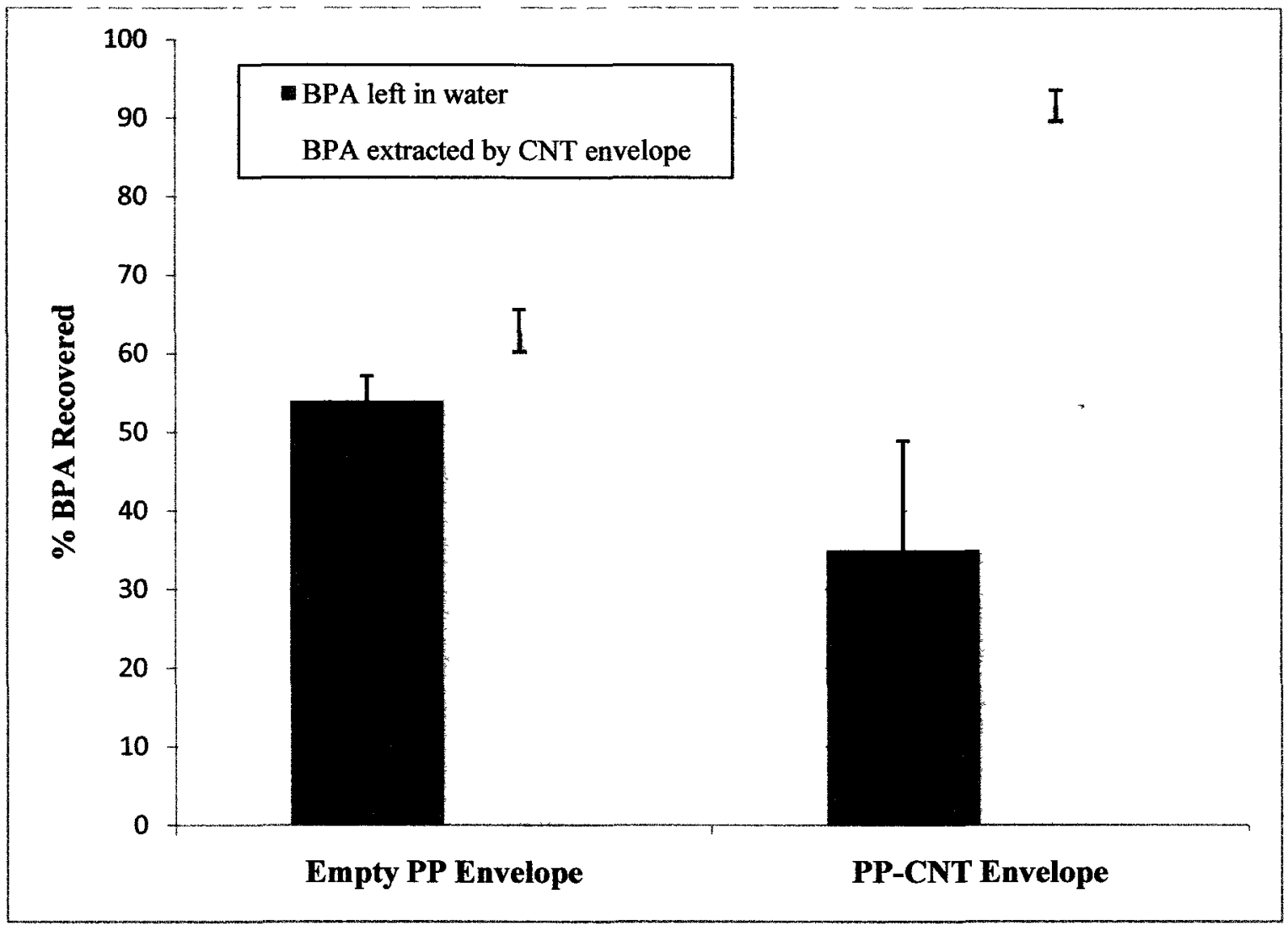

Figure 3-32 BPA recoveries when the rolled PP devices (empty and filled) were conditioned by dipping in methanol prior to use.

It should be noted that the total \% BPA recovered in almost every experiment exceeds $100 \%$. At this time the concentration of BPA left in water was calculated from a calibration curve of BPA in methanol standards. Since the signal of BPA in methanol is greater than in water, the calculated concentrations of the amount of BPA left in water will be too high. However, the trends between conditioning solvents remain the same.

Conditioning the PP-CNT envelopes by any method proved to be more of a hindrance than a benefit. Regardless of conditioning technique or solvent choice, the amount of BPA extracted could not be isolated to only the PP-CNT envelope as the empty PP envelopes also extracted BPA. Consequently, BPA extraction could not be isolated to 
only the carbon nanotubes. Instead, the proposed SPE device acted more like a modified LLE device. Hollow-fiber liquid-phase microextraction (HF-LPME) was developed by Pedersen-Bjergaard and Rasmussen [102] in 1999. In this modified LLE technique, a hollow fiber is dipped in an organic solvent prior to extraction from aqueous solution. The organic solvent forms a barrier at the aqueous interface since the two layers are immiscible. Next, the lumen of the fiber is filled with the extracting solvent. The analytes of interest are extracted from the aqueous phase into the conditioning solvent, and then subsequently to the extracting solvent. While HF-LPME has been successfully used for BPA extraction, the goal of this project was to create an SPE envelope to examine BPA adsorption to carbon nanotubes, not to develop an LLE envelope as an alternative LLE or LF-LPME [102].

In a solid-phase extraction system of an aqueous solution, the interactions that exist are between analyte and aqueous phase, the analyte and sorbent and the sorbent and aqueous phase. In order for adsorption to occur, the interactions between the analyte and sorbent must overcome the interactions between the analyte and water and water and sorbent. In the case of BPA adsorption to PP-CNT envelopes, a conditioning solvent of appropriate polarity needed to be introduced to facilitate any BPA extraction. Introduction of a solvent significantly complicated the system. Although substantial BPA extraction was observed when acetone or methanol was included in the system (by any conditioning method) it could not easily be hypothesized which surface BPA adsorption actually occurred on. By conditioning the envelope in a solvent, a second liquid phase was introduced to the system. When the second liquid phase is a water-miscible solvent 
(when methanol and acetone are considered), mixing between the organic and aqueous phases will likely occur.

When the initial BPA extraction using unconditioned PP-CNT envelopes was discussed in Section 3.5, Figure 3-23 demonstrated the phase separations during extraction. The aqueous and solid phases were separated by the porous membrane. An insignificant amount of BPA was extracted when the envelopes were not conditioned with organic solvents, likely due to the large contact angle between water and the hydrophobic polypropylene membrane. The potential interactions during extraction when the envelope has been conditioned in a moderately polar solvent are too complex to model. When an organic solvent is added to the system, the boundary between aqueous, organic and solid phases is not as distinct. Initially, the conditioning solvent fills the envelope pores as shown in. The presence of the solvent will facilitate water diffusion (and thus BPA) through the membrane, but the solvent will also likely diffuse out of the membrane. Therefore, water, solvent and BPA will be present in different compositions in every phase in the system (not illustrated). Most of the conditioning experiments indicate a small increase in extraction when the envelope is filled with carbon nanotubes, but it remains unclear where BPA sorption sites exist when CNTs are not part of the system. It has been demonstrated previously that polymeric phases are capable of absorbing organic analytes, and it is possible that BPA is being absorbed by the polypropylene membrane as well. Furthermore, although BPA adsorption has been demonstrated when the envelope was filled with carbon nanotubes, it is possible that the organic solvent introduced maybe also be adsorbing to the carbon nanotubes. Chemisorption of acetone on carbon 
nanotubes has been shown by Chakrapani et al. [103], particularly at defect sites along the carbon nanotube surface. It has been demonstrated by Tang [104] that methanol also adsorbs to defect sites. All of potential interactions between BPA, water, solvent, membrane and CNTs make envelope conditioning impossible to model. Therefore, the change to a less hydrophobic membrane was necessary to avoid envelope conditioning and to understand BPA adsorption to carbon nanotubes more fundamentally.

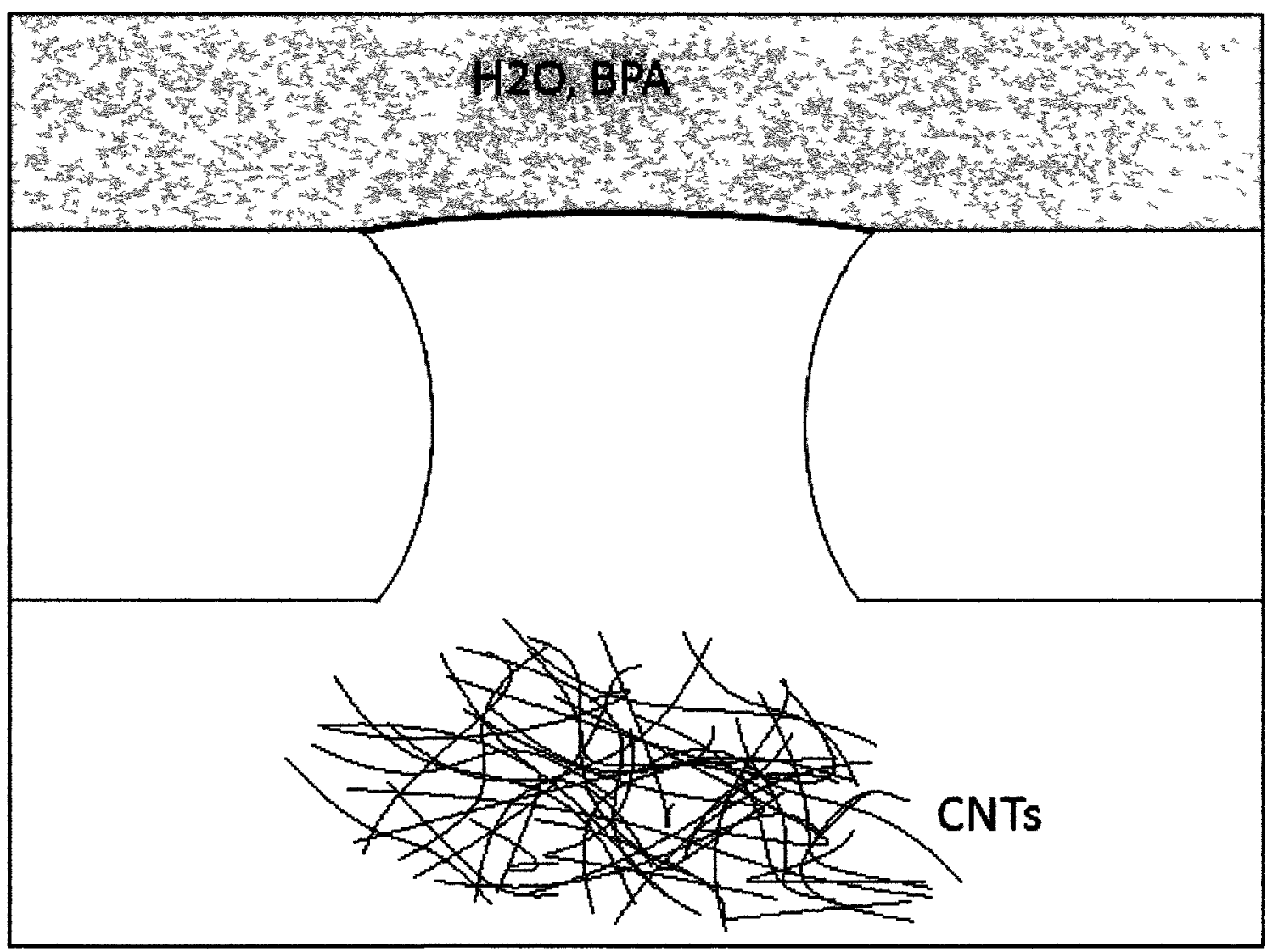

Figure 3- 33 A schematic of the cross-section of a PP-CNT envelope when a conditioning solvent has been introduced to the extraction system. If the solvent is miscible with water, mixing will likely occur both inside and outside the PP-CNT envelope. 


\subsection{Initial BPA Extraction Using PVDF-CNT Envelopes}

The need to find a more hydrophilic membrane to fabricate CNT envelopes was addressed by preparing envelopes from Durapore $($ polyvinylidene fluoride) filter discs. Initial BPA extraction using PVDF-envelopes was performed with similar extraction conditions to the initial extraction using PP-CNT envelopes; however some extraction parameters were changed. Adsorption and desorption times, and water and methanol volumes were maintained for the same reasons described for extraction using the PPCNT envelopes. The concentration of BPA in aqueous solution was decreased to 250 $\mathrm{ng} / \mathrm{mL}$ since the HPLC sensitivity increased after cleaning the column with organic solvents and replacing the lamp.

Unlike the polypropylene membrane, the polyvinylidene fluoride membrane was hydrophilic and when the PVDF-CNT envelope was submerged in solution, it began to fill with water. Over time as more water entered the envelope it became more dense and started to sink to the bottom of the vial. This also occurred during desorption when the PVDF-CNT envelope filled with methanol. As a result the PVDF-CNT envelope would come into contact the stir bar during adsorption or desorption. This was not an issue during some extractions; however other PVDF-CNT envelopes caught on the edge of the stir bar and ripped open, dispersing CNTs into solution. Also, on a few occasions, the PVDF-CNT envelope managed to get trapped underneath the stir bar so that it was lying flat on the bottom of the vial and the stir bar stirred directly on top of it. Consequently it was very difficult to achieve reproducible results and to determine exactly how much BPA the PVDF-CNT envelope was capable of extracting. To overcome this issue, the 
PVDF-CNT envelope was suspended in solution using a nylon twist-lock cable fastener which was successful at holding the PVDF-CNT envelope in place throughout extraction.

Twist-lock cable fasteners were used to suspend both PVDF-CNT envelopes and empty PVDF envelopes in solution for initial BPA extraction with envelopes made from the polyvinylidene fluoride membrane. Again, BPA extraction using the filled CNT envelope was compared against a blank PVDF envelope to determine if the membrane also acted as a sorbent. Five envelopes of each type (empty or filled) were used for separate extractions to determine the reproducibility of the process. Figure 3-34 compares the average of the five PVDF-CNT trials with the average of the results from four blank PVDF envelopes (the results from one extraction were omitted due to contamination). It can be seen that the PVDF-CNT envelopes were capable of extracting BPA from aqueous solution, with an average recovery of $73.21 \%$. The hydrophilic nature of the PVDF membrane facilitated BPA transport through the pores of the envelope to present an opportunity for adsorption to the surface of the carbon nanotubes, unlike the PP-CNT envelope. Figure 3-34 also indicated sorption of BPA to the membrane itself, which was hypothesized could happen, as mentioned previously. 


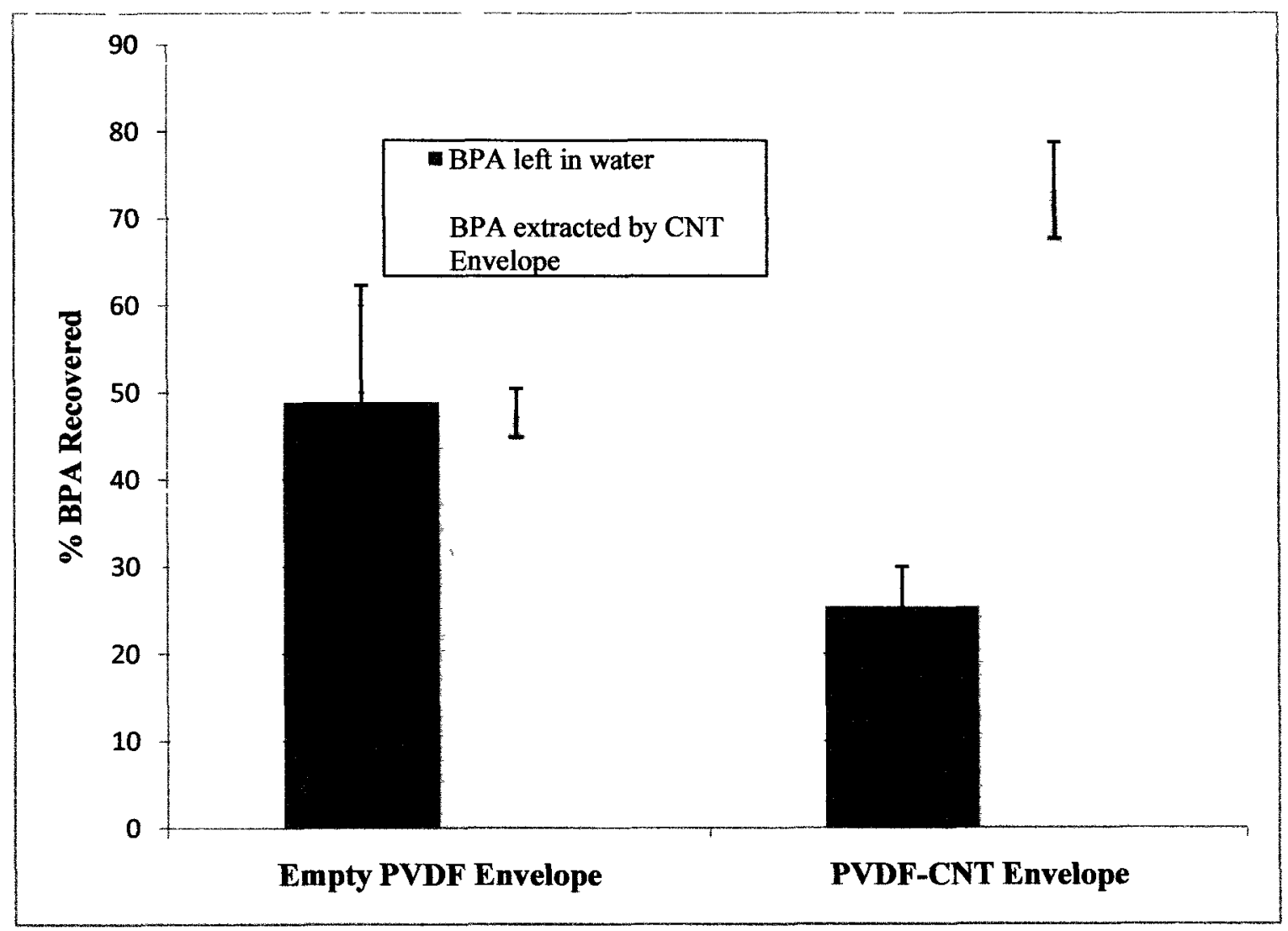

Figure 3-34 BPA extraction using envelopes prepared from the polyvinylidene fluoride membrane. Extraction efficiencies are compared for empty envelopes and envelopes filled with carbon nanotubes.

Figure 3-34 shows the average BPA recovery over five extractions performed with separate envelopes. The precision between recoveries was calculated as the standard deviation from the mean recovery, which is represented by the error bars shown on the chart. The error shown demonstrates moderate fluctuations in BPA recovery from one PVDF-CNT envelope to the next. It is not surprising that the standard deviation between aqueous samples is larger than the methanol extracts since chromatograms of aqueous injections are generally not as resolved and harder to integrate. As well as discrepancy between extractions, instrument error will contribute to the error associated with each measurement. To illustrate variations in extraction efficiency from one envelope to the 
next, the five extractions that are represented by the average (shown in Figure 3-34) are shown individually in Figure 3-35.

Figure 3-35 displays BPA recovery from each extraction using five separate PVDF-CNT envelopes. The amount of BPA recovered ranges from one extraction to the next by about $13 \%$. Aside from chromatographic error, differences in recovery may be the result of several extraction parameters. Although each PVDF-CNT envelope is filled with an average of $6.45 \mathrm{mg}$ carbon nanotubes this mass ranged from $6.0-7.0 \mathrm{mg}$ for each envelope. Furthermore, when the envelopes were prepared, the carbon nanotubes sometimes scattered across the membrane due to the effects of static when the second membrane was placed on top of them. As a result, some CNTs were trapped in the seal between the two membranes and some even scattered outside of the area to be sealed. Therefore, the amount of CNTs in between envelopes could be affected by more than just weighing variations. Although the extractions were performed at room temperature, some of the stirring plates were warm from the motor inside which could have affected the amount of BPA adsorbed. 


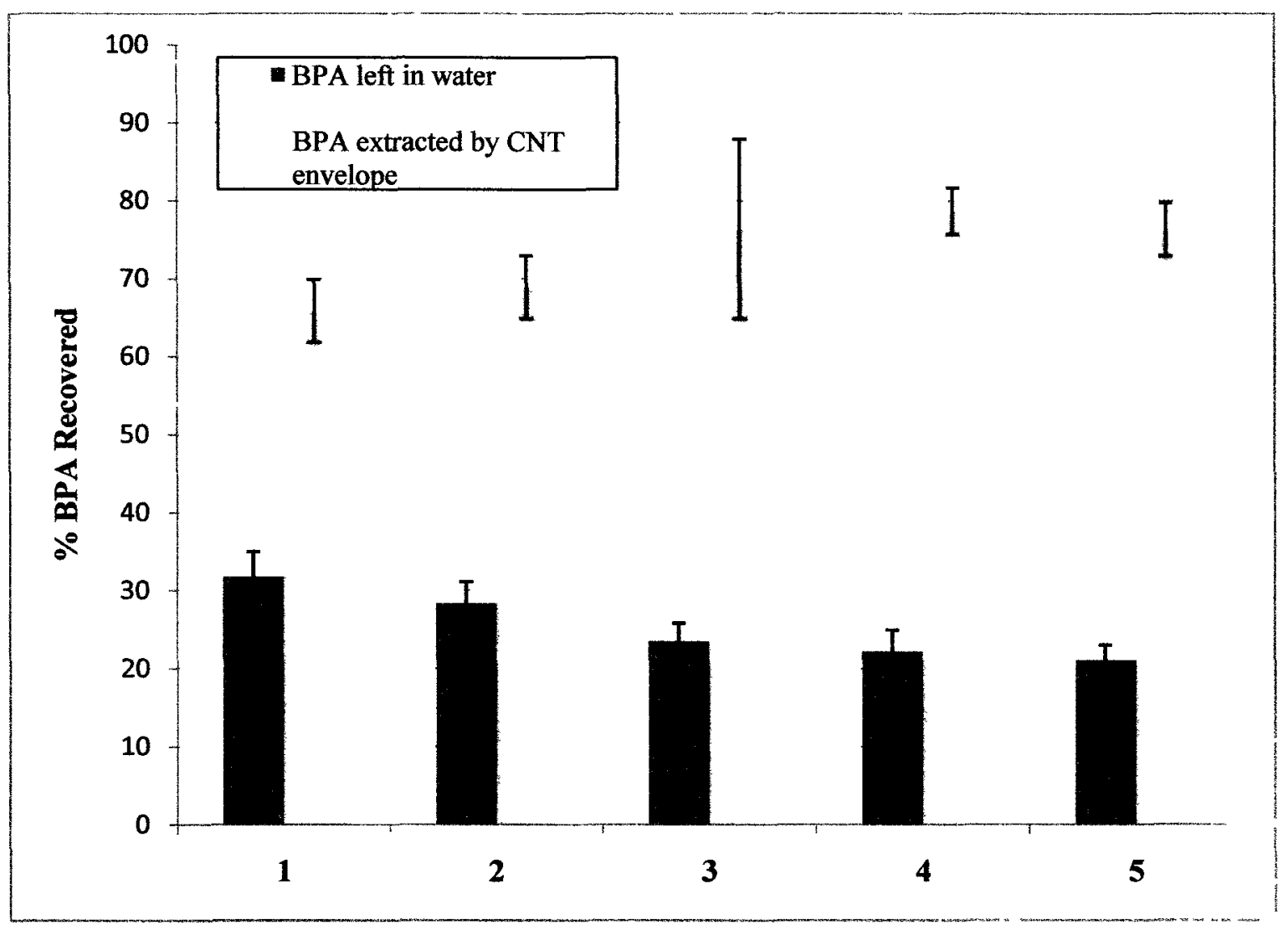

Figure 3-35 Extraction efficiencies from several extractions are compared to show the uncertainty in the amount of BPA recovered from one PVDF-CNT envelope to the next.

Polycarbonate plastic is commonly used to make laboratory equipment and it has been demonstrated BPA leaks from plastic lab equipment during autoclaving [105]. Thus, extreme caution was taken to prevent any BPA contamination during experiments. BPA solutions were only prepared and stored in glassware and all extractions were carried out in glass vials. Although a literature search of the uses of BPA did not provide any evidence that the BPA monomer is used in the production of Teflon or nylon, it was decided that the initial extraction using PP-CNT envelopes should be repeated to ensure that any BPA contamination in the experiments was not the result of leaching from the Teflon stir bar or nylon twist-lock fastener. In addition, coated stir bars have been used 
for SPE applications and it was necessary to ensure the stir bar did not act as a sorbent in this system. The extraction was repeated using the same conditions as those used for the first initial extraction. Three stir bars were simply placed in glass vials for SPE of BPA. Three twist-lock fasteners were suspended in the usual manner (without any CNT envelopes) and extraction was performed with stirring. Three blank PP envelopes and three PP-CNT envelopes were used for extraction as discussed above. The average result of each of these extractions is shown in Figure 3-36.

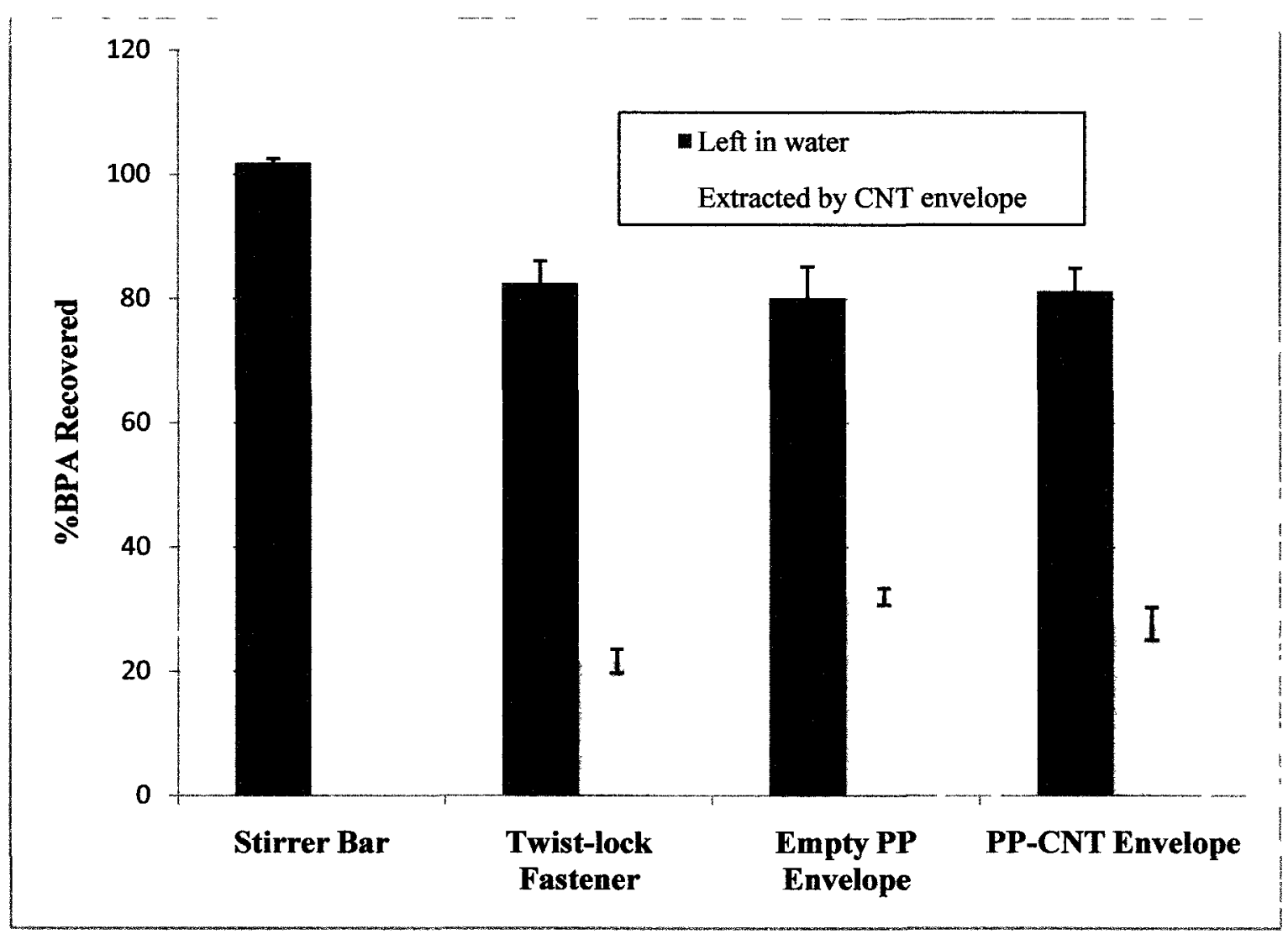

Figure 3-36 BPA recoveries when the intial extraction experiment was repeated using PVDF-CNT envelopes to determine if the other objects present in the system participated in extraction. 
Surprisingly, the results indicated that the nylon twist-lock fastener was capable of extracting BPA from aqueous solution. The amount of BPA removed did not increase significantly when extractions were performed with empty envelopes. Thus, the hypothesis that the PP membrane was capable of sorbing BPA from the sample matrix was unlikely. No interactions occurred between BPA and the hydrophobic membrane. Instead, the extraction observed using blank PP envelopes in the initial experiment was caused by the presence of a twist-lock fastener. When the \% BPA recovered using only twist-lock fasteners (or empty PP envelopes since they are the same) was compared to the amount of BPA recovered using filled PP-CNT envelopes, it was observed that the presence of CNTs did not increase extraction efficiency. As discussed in Section 3.7, BPA diffusion through the hydrophobic polypropylene membrane did not occur without the presence of organic conditioning solvents.

Adsorption is normally governed by three interactions: analyte and solvent, analyte and sorbent and solvent and sorbent. The presence of a second sorbent in the form of a twistlock fastener significantly complicates the extraction system since interactions between the second sorbent and analyte and second sorbent and solvent are introduced. When the PP-CNT envelope was suspended using a twist-lock, competition was created between BPA adsorption to the carbon nanotubes and adsorption to the twist-lock. Although BPA adsorption to the twist-lock was determined to be approximately $20 \%$ in the absence of carbon nanotubes, it could not be assumed this amount would adsorb when competition was created with the carbon nanotubes. To determine how much BPA adsorbed the twist-lock and how much adsorbed to the nanotubes, BPA extraction was performed 
again in the same fashion as before, except the twist-lock and PVDF-CNT envelopes were desorbed separately. Figure 3-37 shows the distribution of BPA between the two sorbents. In the presence of carbon nanotubes, BPA adsorption to the twist-lock fastener actually decreased. BPA likely has a higher affinity for carbon nanotubes than nylon. However, the use of a twist-lock fastener substantially complicated the extraction system making it extremely challenging to model. Those experiments that were performed by suspending the envelope with a twist-lock fastener were either redone or adjusted by $\sim 12 \%$.

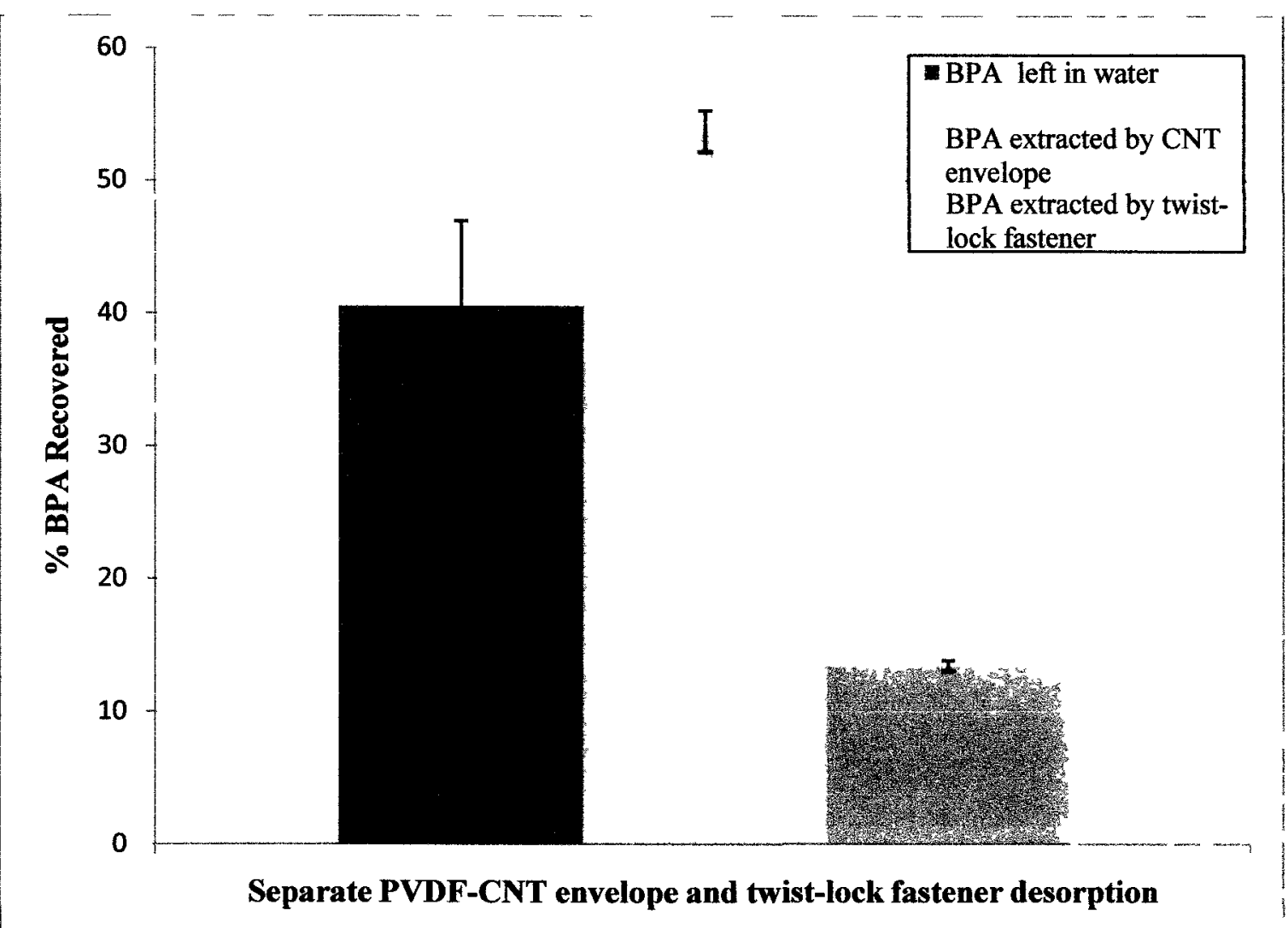

Figure 3-37 The amount of BPA adsorbed to the twist-lock fastener was determined when the PVDF-CNT envelope and twist-lock were separated for desorption during extraction. 
Other ways to suspend the PVDF-CNT envelope were explored and an alternative arrangement was constructed. A small piece of Teflon tubing was cut from the bulk tube and a slit was cut through the center, perpendicular to the plane of the tube. Caution was taken to only cut a slit through about half of the tube and not all the way through. The slit could then be pried apart with pliers and the PVDF-CNT envelope was fitted inside. A piece of Teflon wire was strung through the center of the tube to suspend it in aqueous solution. Figure 3- 38 depicts PVDF-CNT envelopes suspended with a twist-lock fastener and a piece of Teflon tubing.
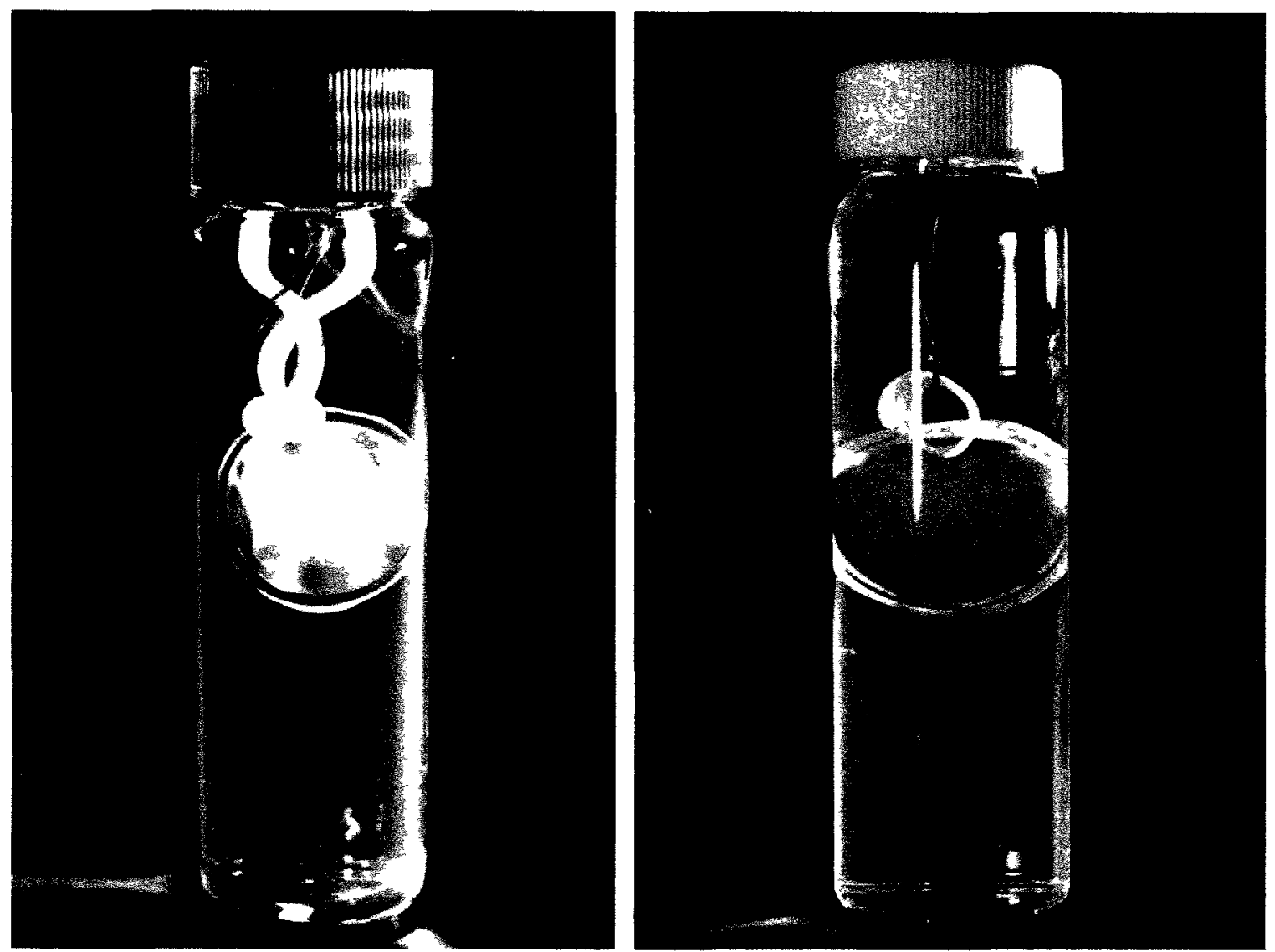

Figure 3- 38 PVDF-CNT envelopes suspended in water using a twist-lock fastener (shown left) and a piece of Teflon tubing (shown right). 
Prior to use as a method of suspending PVDF-CNT envelopes, BPA extraction was performed using only the Teflon tube device to ensure the same issues did not arise as with the twist-lock fastener. The average of three extractions using only the Teflon tubing is shown in Figure 3-39. Extraction using the Teflon tube indicated than no BPA adsorbed to the Teflon and thus it could be used to successfully suspend the envelope without participating in the extraction system.

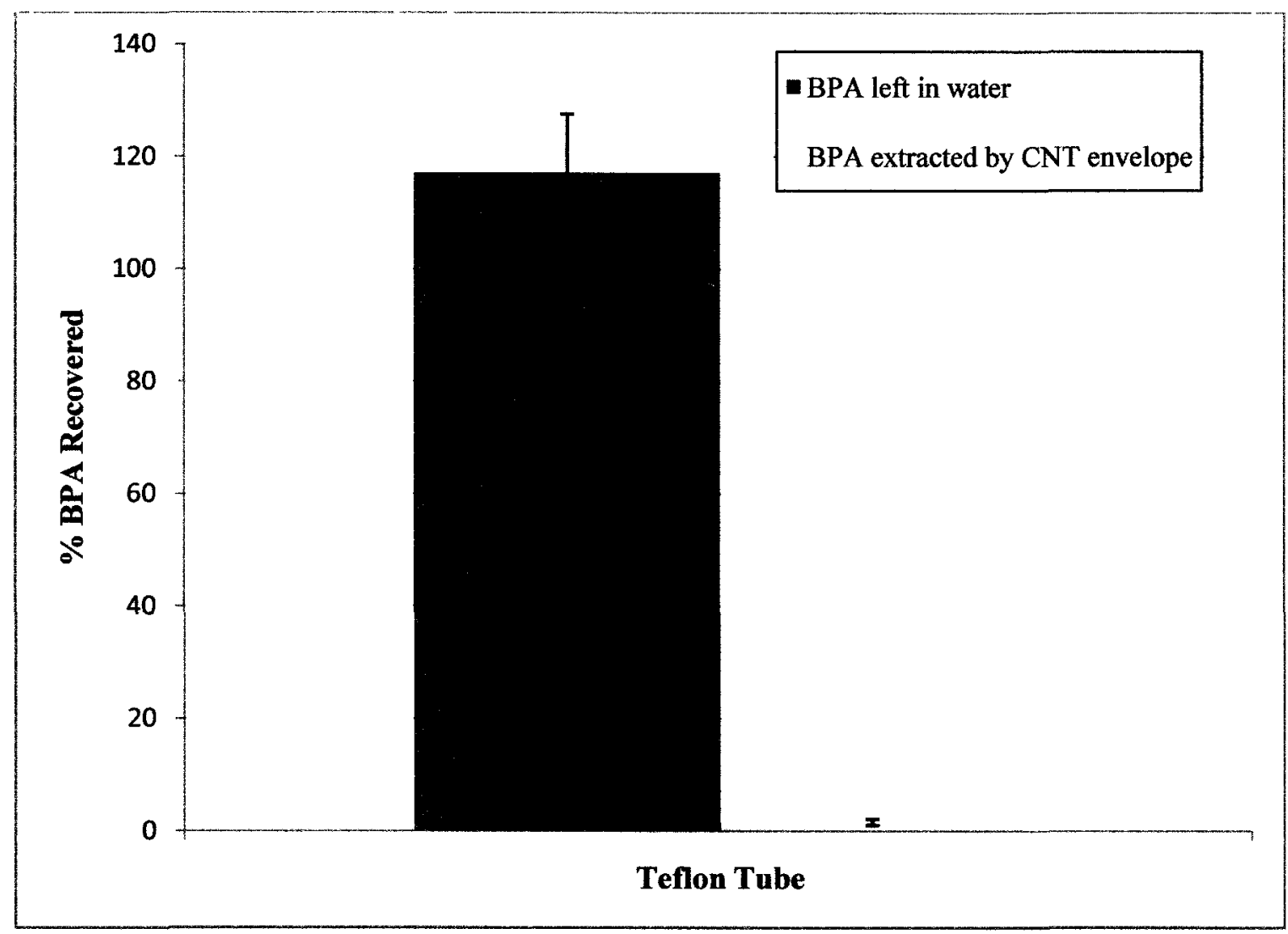

Figure 3-39 The extraction capability of the Teflon tube apparatus was explored before using it to suspend PVDF-CNT envelopes to ensure the problems associated with twistlock fasteners did not arise.

One of the problems associated with SPME fibers is the observance of carryover effects between extractions [72]. Successive desorptions were performed with a $\mu$-SPE device 
(Basheer et al.) and no analytes were detected in the second desorption [97]. In order to test for carryover effects from a PVDF-CNT envelopes, BPA extraction using CNT envelopes was performed with the same extractions conditions as previously described but the envelopes were desorbed in a second aliquot of methanol to determine if any BPA was retained in the envelope after the first desorption. After any BPA in the envelope was eluted with methanol, the envelope was transferred to another $15 \mathrm{~mL}$ of pure methanol for 1 hour. The average results of the first and second desorption from extraction with three PVDF-CNT envelopes are shown in Figure 3-40. It can be seen in this figure that a small quantity of BPA was recovered from the second desorption, however the amount recovered $(\sim 2.8 \%)$ is insignificant compared to the amount of BPA extracted in the first desorption. Furthermore, extractions using PVDF-CNT envelopes are subject to considerable uncertainty based on instrumental and experimental errors. When the PVDF-CNT envelope placed was in solution it immediately began to inflate. Thus, when the PVDF-CNT envelope was removed from methanol, there would be a small volume of methanol present in the envelope. If the envelope was used for extraction then when it is removed from methanol after desorption, BPA will be present in the small volume that remained in the envelope. As the methanol evaporates from the envelope, any BPA molecules left in the envelope may adsorb to the CNT surface once again. When the extraction efficiencies of five PVDF-CNT envelopes were compared (shown in Figure 3-35), the amount of BPA recovered varied by about $13 \%$. Thus the amount of BPA retained in a CNT envelope will also fluctuate. However, the sum of \% BPA left in water and $\%$ BPA extracted by the envelope has been approximately $100 \%$ for each extraction, which indicates all BPA is accounted for after only one desorption. 
If carry over effects are of significant concern, the PVDF-CNT envelope can simply be placed in a small volume of methanol to remove any additional BPA retained by the envelope prior to the next extraction.

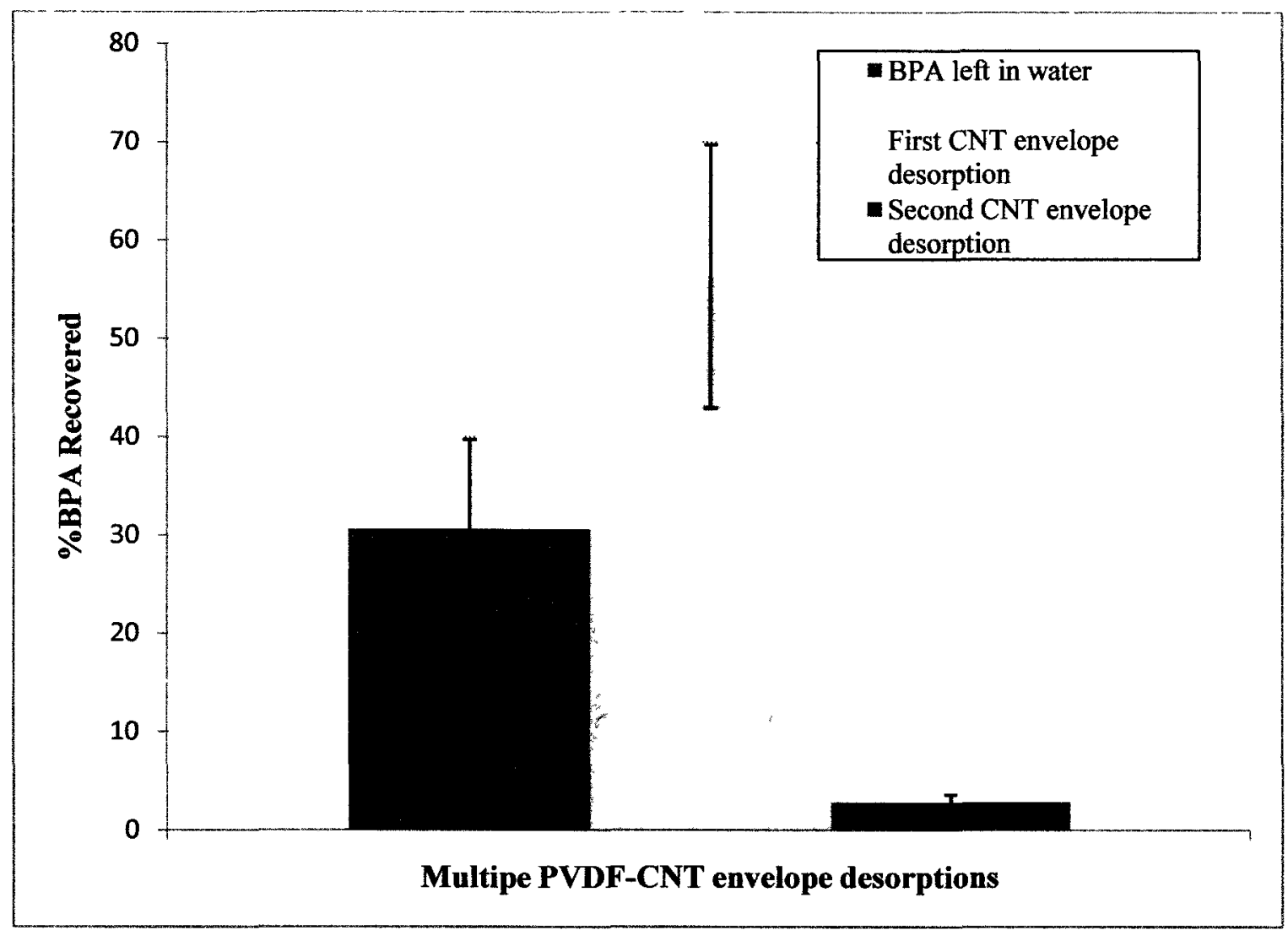

Figure 3-40 Two successive desorptions were performed on the same PVDF-CNT envelopes to determine if any BPA was retained in the envelope.

To test the reusability of a single PVDF-CNT envelope, one envelope was used to perform repeat BPA extractions from aqueous solution. The results of this study, as shown in Figure 3-41, indicate that the PVDF-CNT envelope could easily be used for repeat extractions without analyte loss. With the exception of the second extraction, all BPA recoveries were within one standard deviation of one another. Furthermore, the 
concentration of BPA recovered does not increase over time, which indicates analyte carryover from one extraction to the next was not of concern.

The number of successive trials performed with a single envelope was dependent on the longevity of the PVDF-CNT envelope, specifically on the durability of the membrane. BPA recovery from nine trials is shown in Figure 3-41 and the envelope could have likely been used for several more extractions since no evidence indicates otherwise. The reusability of a $\mu$-SPE device was also examined and Basheer et al. [97] indicated that the extraction efficiency was not compromised for up to 30 analyses. The durability of the polypropylene membrane was the only factor that affected successive extractions [97].

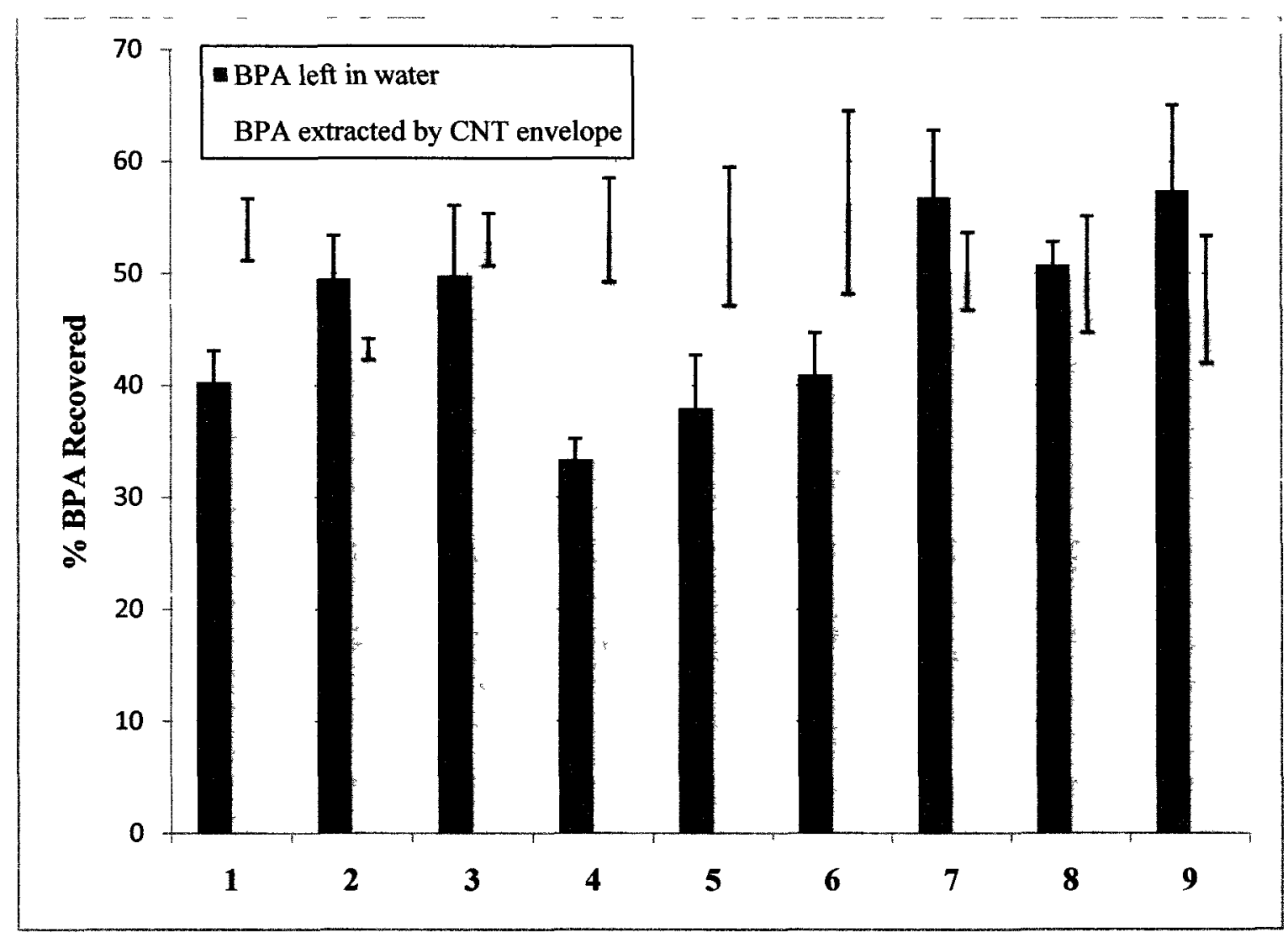

Figure 3-41 Reusability of a single PVDF-CNT envelope was explored over successive BPA extractions 


\subsection{BPA Adsorption to PVDF-CNT Envelopes}

Adsorption can be defined as the attachment of one substance to the surface of another substance. Adsorption can be physical (physisorption), where van der Waals interactions exist between the analyte and sorbent or chemical (chemisorption), where a chemical bond is formed between the analyte and substrate. During adsorption to a solid phase, a relationship exists between the adsorbent, analyte in solution and analyte adsorbed to the adsorbent. This relationship can be modelled by a chemical reaction where the adsorbent and analyte can be thought of as reactants and the analyte attached to the solid phase can be thought of as the product, as shown below. It has been assumed extraction in this reaction is being performed in aqueous media.

Analyte $_{(\text {(q.) }}+$ Adsorbent $_{(\text {aq.) }} \rightleftarrows$ Analyte-adsorbent complex $_{(\text {aq.) }}$

As can be seen from this schematic, an equilibrium exists between the free analyte and adsorbent in solution and the analyte- adsorbent complex. Where this equilibrium lies is dependent on the analyte of interest and type of sorbent used for extraction. This relationship can be used to model BPA extraction from aqueous solution using carbon nanotubes as shown below. The adsorbent has been labeled only as CNTs since it has been shown that the PVDF membranes do not actively participate in extraction.

$$
\mathrm{BPA}_{(\mathrm{aq})}+\mathrm{CNT}_{(\mathrm{aq})} \underset{k_{-1}}{\stackrel{k_{1}}{\rightleftarrows}} \mathrm{BPA}-\mathrm{CNT}_{(\mathrm{aq})}
$$

The rate of formation of the BPA-CNT complex can be determined by the following equation: 
$\frac{d[B P A-C N T]}{d t}=-\frac{d[B P A]}{d t}=k_{1}[B P A][C N T]$

This equation is first-order in BPA and first-order in CNTs and thus has an overall order of two. However, before significant adsorption has occurred and when [CNT] $>>>$ [BPA], the adsorption can be considered to be a pseudo first-order process since [CNT] is essentially constant at high concentrations. The statement $[\mathrm{CNT}] \gg>[\mathrm{BPA}]$ is really a reflection of the number of moles of BPA present in the system and the number of available adsorption sites on the CNTs. Thus when the mass of CNTs used is sufficient enough that the number of adsorption sites greatly exceeds the number of molecules adsorbing, the rate of formation of the BPA-CNT complex can be considered pseudo-first order and can be simplified to the expression shown below:

$\frac{d[B P A-C N T]}{d t}=-\frac{d[B P A]}{d t}=k_{1}^{\prime}[B P A]$

Where $k_{1}^{\prime}=k_{1}[C N T]$

\subsubsection{BPA Adsorption Rate on PVDF-CNT Envelopes}

\subsubsection{BPA Adsorption with Stirring}

To study the adsorption rate of BPA to CNT envelopes, several extractions were carried out with varying adsorption times from $\sim 1-45$ hours and the results are shown in Figure 3-42. Adsorption usually occurs quickly at first since so many vacant sites exist on the adsorbent, then slows as the analyte concentration on the adsorbent increases, until equilibrium occurs, a leveling off of the adsorption curve. Upon equilibrium the amount 
of analyte adsorbed will not increase regardless of the amount of time the sorbent and analyte are in contact with one another. Overall, the time required for BPA adsorption to reach equilibrium was slow. After an hour, only $12 \%$ of the BPA was extracted by the PP-CNT envelope and the adsorption curve did not begin to plateau until about 9.25 hours. The average \% BPA extracted at equilibrium was $54 \%$. To ensure equilibrium was obtained an adsorption time of 24 hours was set for all further experiments.

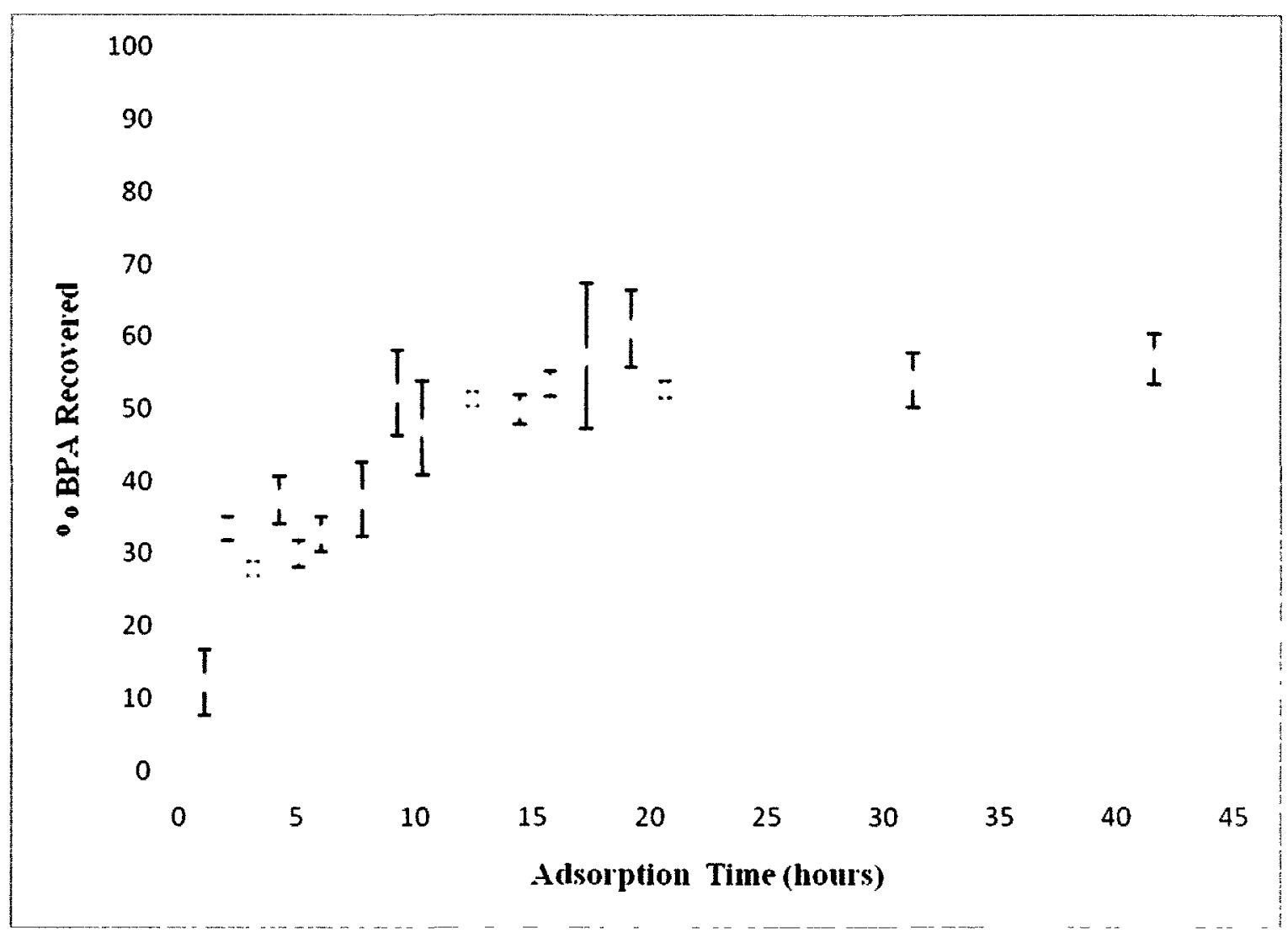

Figure 3-42 Adsorption rate of BPA to CNT envelopes with stirring

The extraction of several organophosphorous pesticides using the $\mu$-SPE device, designed by Basheer et al. [97], reached equilibrium for all pesticides within 30 minutes and extraction actually decreased for some analytes after this time. Furthermore, a $\mu$-SPE 
device filled with $\mathrm{C}_{18}$ was used for extraction of ketoprofen and ibuprofen from wastewater and equilibrium was observed after 50 minutes [98]. However, the hydrophobic membrane which was conditioned in organic solvents was used to make the $\mu$-SPE devices used in both these studies and the authors indicated the adsorption rate was much slower without the use of conditioning solvents.

The concentration of BPA in the CNT envelope can be assumed to be the concentration of BPA on the nanotubes since it has been shown that BPA does not adsorb to the polyvinylidene membrane in Section 3.9. Therefore, the equilibrium obtained between BPA left in aqueous solution and BPA adsorbed to the CNTs can be described by:

$$
K_{e x}=\frac{a_{B P A, C N T}}{a_{B P A, H_{2} O}}
$$

where $K_{e x}$ represents the equilibrium constant, or partition coefficient, and $a_{B P A, e n v}$ and $a_{B P A, H_{2} O}$ represent the activities of BPA on the CNTs and left in aqueous solution, respectively. The activity represents the effective concentration of a solution and is equal to the product of activity coefficient and concentration:

$$
K_{e x}=\frac{\gamma_{B P A, C N T} \bullet[B P A]_{C N T}}{\gamma_{B P A, H_{2} O} \bullet[B P A]_{H_{2} O}}
$$

In this equation, $\gamma$ is the activity coefficient of BPA on the CNTs or in aqueous solution which measures deviation from ideal behaviour and [BPA] represents the concentration of BPA either adsorbed to the nanotubes or left in water. In dilute solutions and at much less than monolayer coverage on adsorbent surface, the activity coefficient is equal to 1 , 
since behaviour is considered to be ideal. Thus, the equilibrium expression can be simplified to a ratio of concentrations:

$$
K_{e x}=\frac{[B P A]_{C N T}}{[B P A]_{H_{2}} \mathrm{O}}
$$

The concentration of BPA on the carbon nanotubes is most appropriately expressed as the fraction of CNT surface covered by BPA molecules which can be expressed as surface area of BPA adsorbed to the CNTs $\left(\mathrm{SA}_{\mathrm{BPA}} \mathrm{CNT}\right)$ over total $\mathrm{CNT}$ surface area available (SA $\mathrm{CNT}_{\mathrm{C}}$ ). The mass of CNTs used was constant in all experiments, except for one investigation, so the total CNT surface area was generally constant. The concentration of BPA left in water is more accurately represented as the aqueous concentration of BPA at equilibrium $\left([\mathrm{BPA}]_{\mathrm{H}_{2} \mathrm{O}}\right.$ ) over the molar concentration of BPA at standard conditions ( $[B P A]_{H_{2} O}^{0}$ ) (which is equal to $\left.1 \mathrm{M}\right)$. Thus, the equilibrium expression can be altered to reflect these changes:

$$
K_{e x}=\frac{\frac{S A_{B P A, C N T}}{S A_{C N T}}}{\frac{[B P A]_{H_{2} O}}{[B P A]_{H_{2} O}^{0}}}
$$

The graph of \% BPA adsorbed over time shown in Figure 3-42 depicts the shape of the equilibrium curve and the data from this experiment can be used to calculate the value of the equilibrium constant. To determine the fraction of BPA adsorbed to the CNTs compared to total CNT surface available, the surface area of a single BPA molecule must be determined first. 
The dimensions of a BPA molecule when it is assumed to be a two-dimensional molecule are shown below:

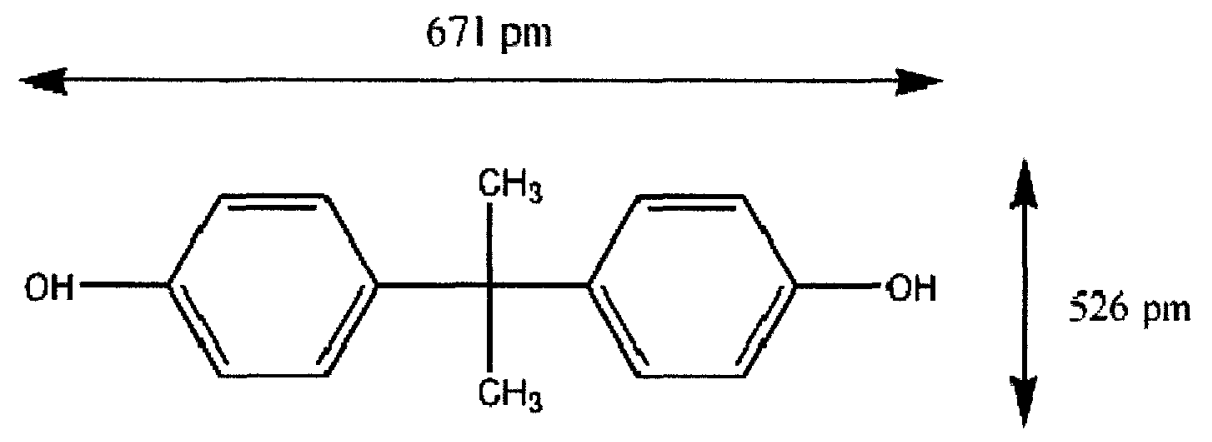

Figure 3-43 Dimensions of BPA.

BPA was assumed to be rectangular and the surface area of the molecule was calculated to be $3.53 \times 10^{-19} \mathrm{~m}^{2} /$ molecule, or $2.13 \times 10^{5} \mathrm{~m}^{2} / \mathrm{mol}$. As stated, the mass of BPA extracted by the CNTs can be determined from the calibration curve. The rate of BPA adsorption to CNT envelopes began to level off at 9.25 hours. At this time, the amount of BPA extracted by the CNT envelope was $5.34 \mu \mathrm{g}$. Using this point as an example, the mass of BPA extracted can be divided by the molar mass of BPA to determine the number of moles of BPA, $2.34 \times 10^{-8}$. Thus, the surface area of BPA extracted was determined to be $4.97 \times 10^{-3} \mathrm{~m}^{2}$.

The surface area of CNTs at monolayer coverage cannot be calculated from the surface area of a single CNT since the molar mass of CNTs cannot be calculated. Carbon nanotubes range significantly in length and diameter and as such the molar mass is not constant from one CNT to the next. Instead the surface area of the CNTs used can be determined by multiplying the mass of CNTs in the envelope by the measured surface area. As mentioned, in all but one experiment, the mass of CNTs used was $\sim 6.45 \mathrm{mg}$. 
The surface area of the long MWCNTs used in almost every experiment was determined to be $220.441 \mathrm{~m}^{2} / \mathrm{g}$, as shown in Table 3-2. Thus, the surface area of CNTs used in one envelope was $1.42 \mathrm{~m}^{2}$.

The concentration of BPA left in aqueous solution in $\mathrm{ng} / \mathrm{mL}$ can also be determined from the calibration curve and easily converted to mol/L using the molar mass of BPA. Thus, the equilibrium constant can be determined:

$$
\begin{aligned}
K_{e x}= & \frac{\frac{S A_{B P A, C N T}}{S A_{C N T}}}{\frac{[B P A]_{H 2 O}}{[B P A]_{H 2 O}^{0}}} \\
K_{e x}= & \frac{\frac{4.97 \times 10^{-3} \mathrm{~m}^{2}}{1.42 \mathrm{~m}^{2}}}{\frac{6.20 \times 10^{-7} \mathrm{~mol} / \mathrm{L}}{1 \mathrm{~mol} / \mathrm{L}}} \\
K_{e x}= & 5649
\end{aligned}
$$

When BPA adsorption to PVDF-CNT envelopes was examined over time, the curve did not reach equilibrium until 9.25 hours, as discussed. The equilibrium constant can be calculated at each point in the curve at and after equilibrium has been reached. In theory, if the adsorption rate reached equilibrium, the value of the partition coefficient for each point at equilibrium should be the same. In contrast, in Figure 3-44, the equilibrium constant appeared to increase over time initially and then level off. This may indicate BPA adsorption to the CNTs was not actually at equilibrium at 9.25 hours, although it appeared to be in Figure 3-42. However, the plot of equilibrium constant versus adsorption time indicates adsorption did reach equilibrium shown by a leveling off of the partition coefficients. The adsorption time was designated to be 24 hours for all future 
experiments, and the plot of partition coefficients demonstrates this time was appropriate as adsorption was at equilibrium at this time. The average equilibrium constant can be determined from the partition coefficients at equilibrium and was calculated to be 10000 .

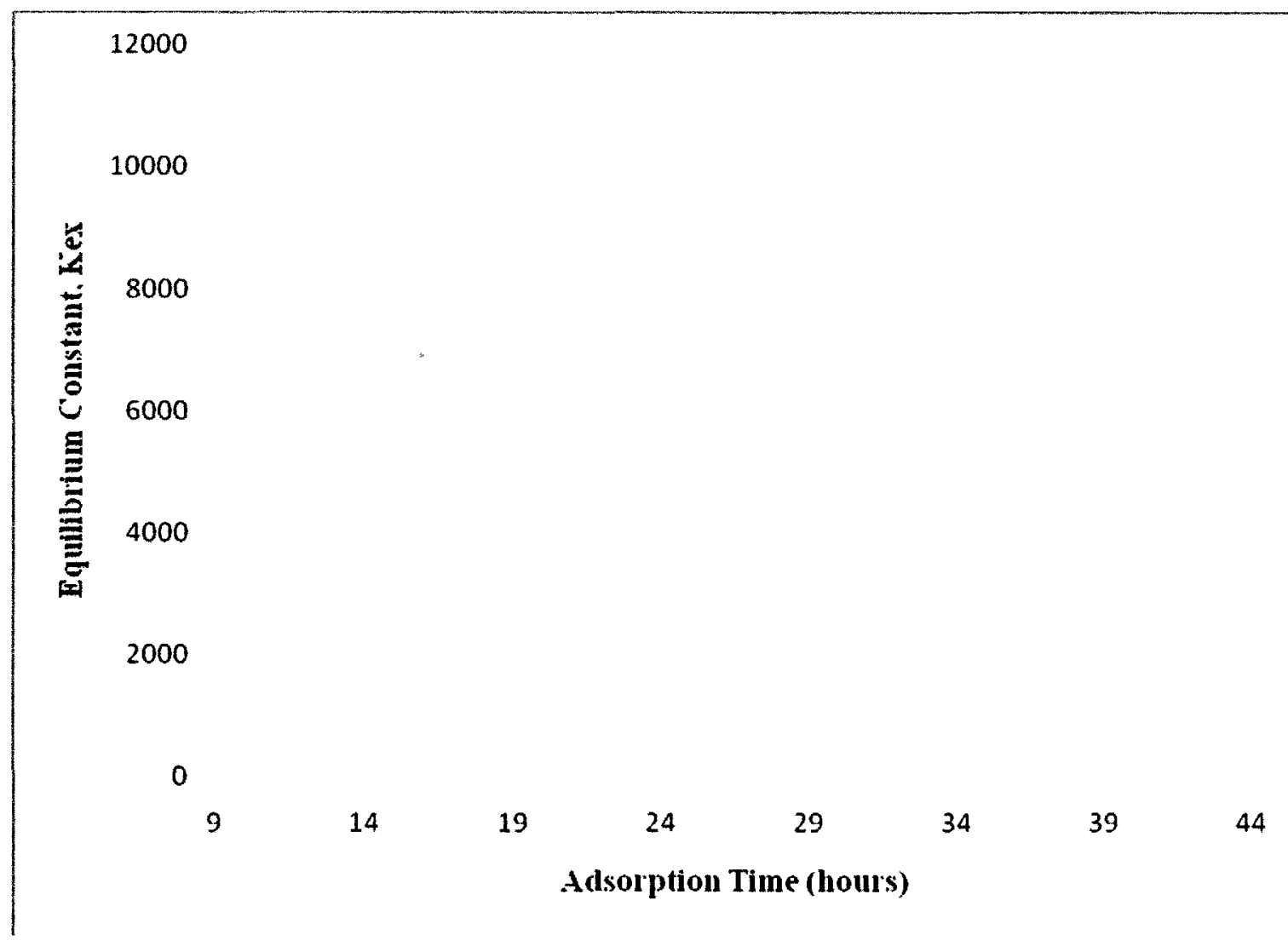

Figure 3-44 Partition coefficients corresponding to each point in equilibrium when the rate of BPA adsorption to CNTs was examined.

\subsubsection{BPA Adsorption with Sonication}

In SPME, agitation conditions promote analyte adsorption to the fiber. Although, sonication has been used primarily since it affords the quickest analysis times, magnetic stirring has also been documented to promote adsorption $[69,70]$. In this work, a single PVDF-CNT envelope was sonicated in water for $30 \mathrm{~min}$ to determine if BPA extraction 
could be obtained using alternate agitation conditions. As mentioned, the durability of the envelope membrane and fragility of the seal affected CNT envelope longevity the most. Harsh sonication conditions caused the envelope to rip easily, dispersing CNTs in solution. When the envelope did not rip, the water in the ultrasonicating bath needed to be cycled continuously to prevent the aqueous BPA solution from heating during extraction. Thus the effects of sonication were only studied once and the results, shown in Figure 3-45, indicate \% BPA recovery was not enhanced in the time frame employed for this trial. Due to the problems associated with sonicating PVDF-CNT envelopes, the use of sonication during BPA extraction was abandoned, and the adsorption rate using sonication was not measured. 


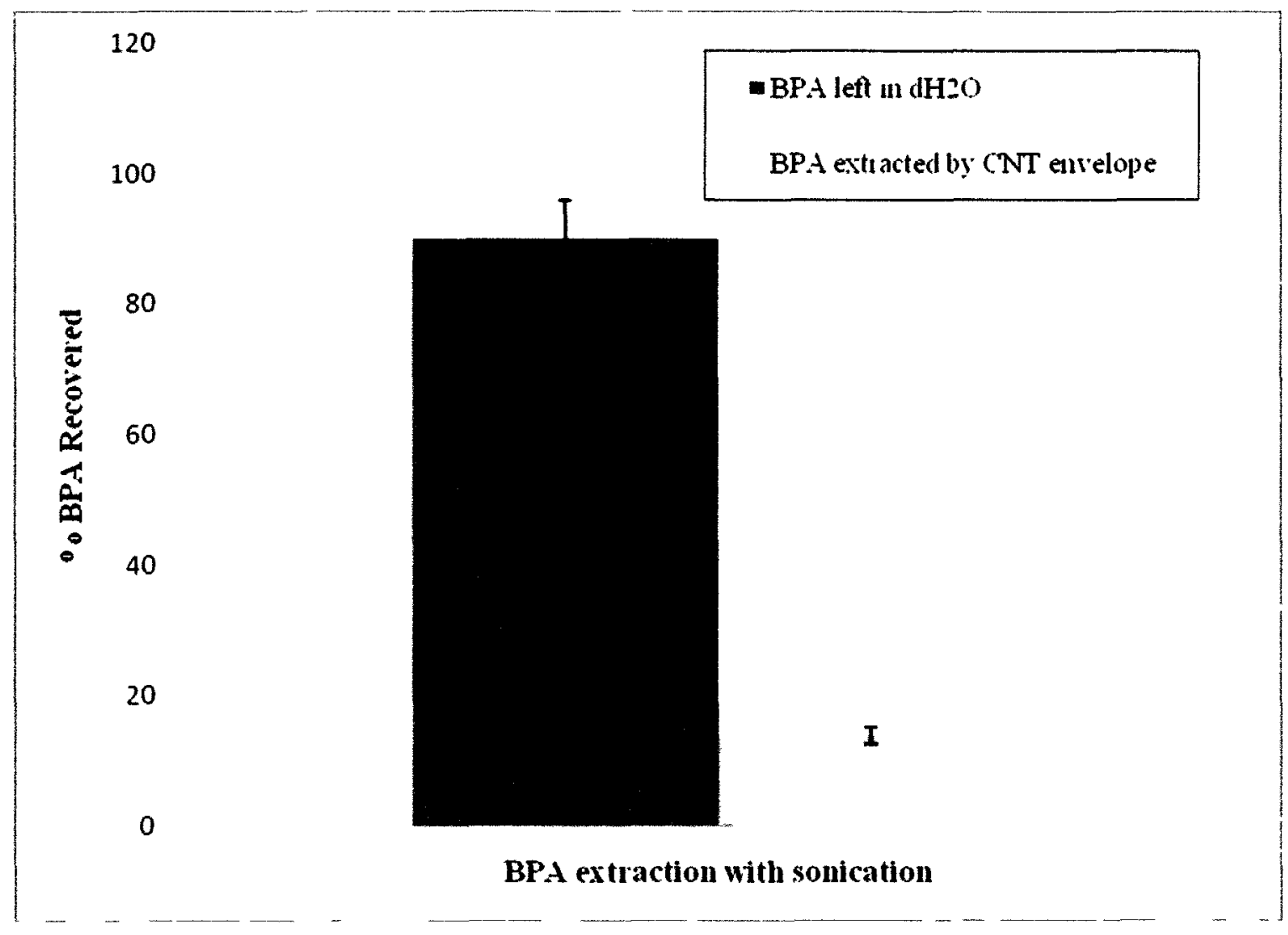

Figure 3-45 BPA extraction usinga single PVDF-CNT when adsoprtion was performed in a sonicator for 30 minutes.

\subsubsection{BPA Adsorption using PVDF-CNT Envelopes made from Membranes with}

\section{Larger Pore Diameter.}

The adsorption rate of BPA to carbon nanotubes using CNT envelopes is significantly slower than adsorption rates reported in other studies. The rate-limiting step in this process is likely BPA diffusion through the pores of the membrane. When the PVDF membrane was selected to make a CNT envelope using a hydrophilic membrane, PVDF membranes were available with pore diameters ranging from $100 \mathrm{~nm}$ to $5 \mu \mathrm{m}$. Bulk CNTs exist as more of an aggregated bundle than individual tubes lying oriented in the direction of the pores and thus it was unlikely that any CNTs would escape from the 
envelope through the pores of the membrane. However to be prudent, PVDF discs with the smallest diameter $(100 \mathrm{~nm})$ were purchased.

To determine if the BPA adsorption rate to carbon nanotubes could be improved if the PVDF-CNT envelope was made from a membrane with a larger pore size, CNT envelopes were also made using a PVDF membrane with pore diameter $0.65 \mu \mathrm{m}$. Although this membrane was made from the same material as those with the smaller pore size, the grade of polymer was different and they were considerably harder to seal with the temperatures afforded by the heat gun. Significant pressure needed to be applied to the heated sealer tool in order to successfully fuse the two membranes. The rate of adsorption was studied in the same fashion as the CNT envelopes with smaller diameter. Figure 3-46 outlines the adsorption curve when the CNT envelopes were made from membranes with larger pore diameter. Figure 3-46 indicates that the larger pore diameter did not accelerate BPA adsorption any more than the membrane with smaller pore diameter. The manufacturer indicated that the porosity of the polyvinylidene fluoride membrane was $70 \%$, regardless of pore diameter, indicating one membrane may have larger pores size but fewer of them. In addition, both membranes had a thickness of 125 $\mu \mathrm{m}$. Diffusion across a concentration gradient is dependent on distance and since the distance travelled through either membrane is the same, the rate of diffusion should not change. 


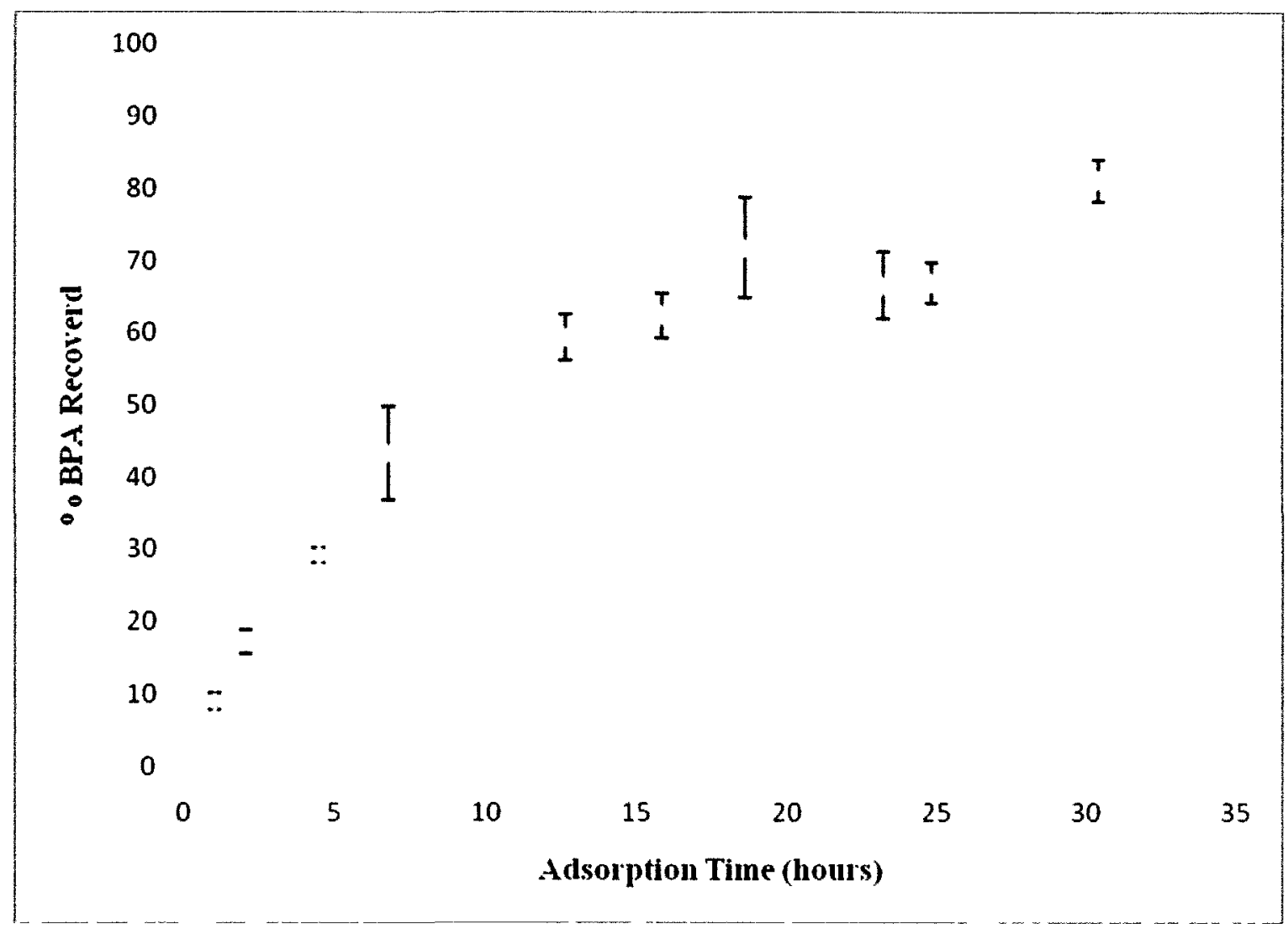

Figure 3-46 BPA adsorption rate to PVDF-CNT envelopes that were prepared from a membrane with an effective pore diameter of $0.65 \mu \mathrm{m}$.

\subsubsection{Adsorption without stirring}

The adsorption rate was also explored without the use of stirring. Stirring was used during all extractions in this work, however in order to examine the effects of temperature on BPA adsorption to CNTs, the adsorption temperature needed to be controlled in a water bath, thus eliminating the use of stirring. As a result, it was important to know how long BPA adsorption took to reach equilibrium without agitation to the system. Extractions were performed in the same manner as those with stirring except the glass vials containing the CNT envelopes immersed in aqueous solution were simply left on the lab bench. Stirring was still employed during BPA desorption from 
PVDF-CNT envelopes. The results presented in Figure 3-47 indicated that BPA adsorption to carbon naontubes was slowed without the use of magnetic stirring. As such, magnetic stirring was employed during adsorption in all experiments, except when the effect of temperature was examined. Without stirring, an adsorption time of 3 days was used to ensure adsorption reached equilibrium.

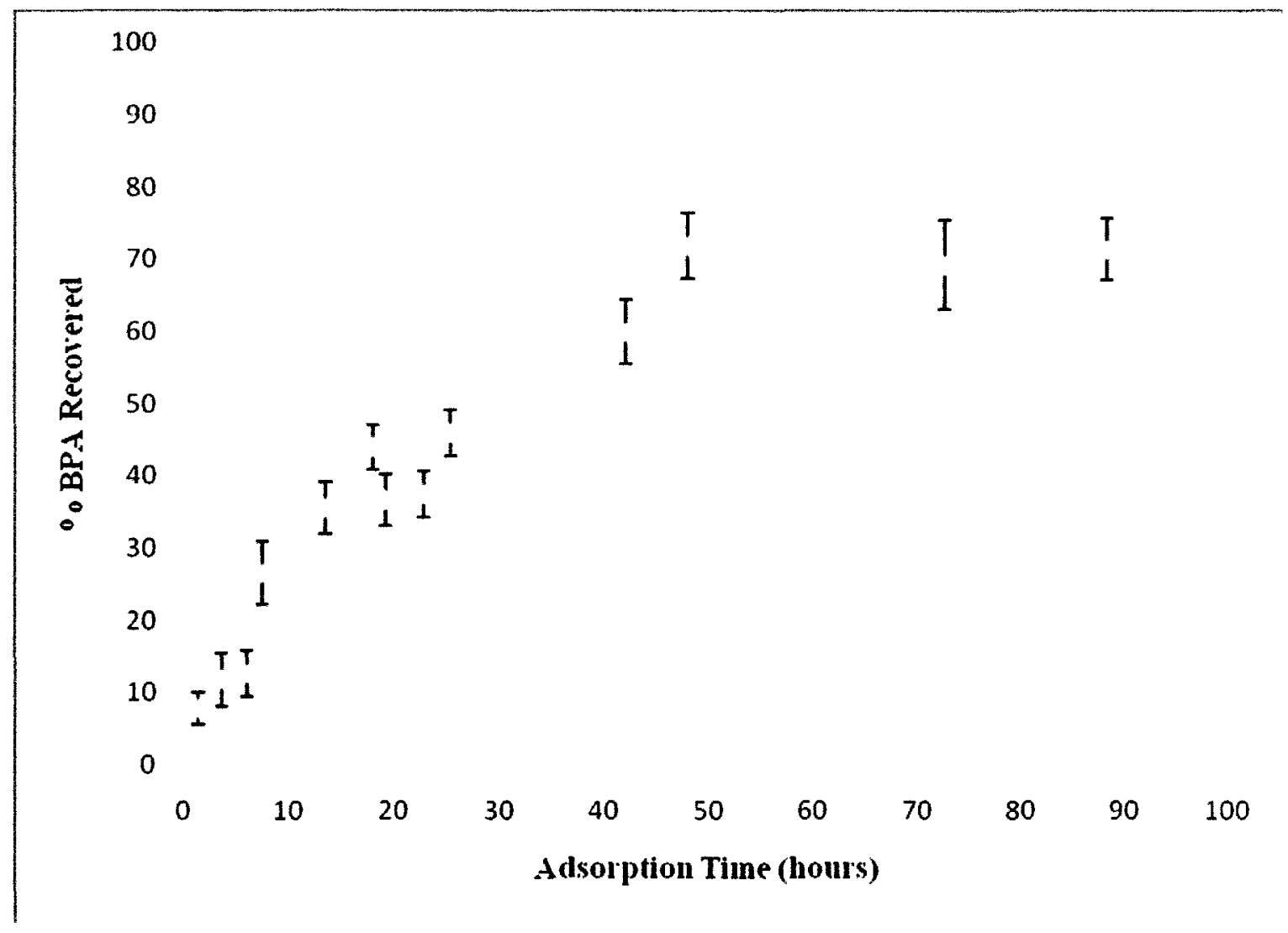

Figure 3-47 Rate of adsorption of BPA to PVDF-CNT envelopes without stirring.

Since adsorption rate is a function of kinetics, and not thermodynamics, the studies of adsorption rate with different pore size and without stirring did not enhance extraction efficiency at equilibrium. 


\subsubsection{Effect of Temperature}

To examine the impact of temperature on BPA extraction, several extractions were performed in a water bath that was maintained at constant temperature. A glass vial containing the PVDF-CNT envelope suspended in aqueous BPA solution was held in the water bath using a clamp and retort stand. The solution could not be stirred as the water bath was significantly larger and deeper than a stirring plate. Consequently, the extraction was performed for a longer period of time ( $\sim 3$ days) to ensure adsorption reached equilibrium. Desorption was performed as usual; in methanol at room temperature on a stirring plate. Figure 3-48 shows the effect of temperature on BPA recovery using PVDF-CNT envelopes. As demonstrated, extraction efficiency actually increased at elevated temperatures. This result is counter-intuitive since analyte adsorption generally decreases at higher temperatures. 


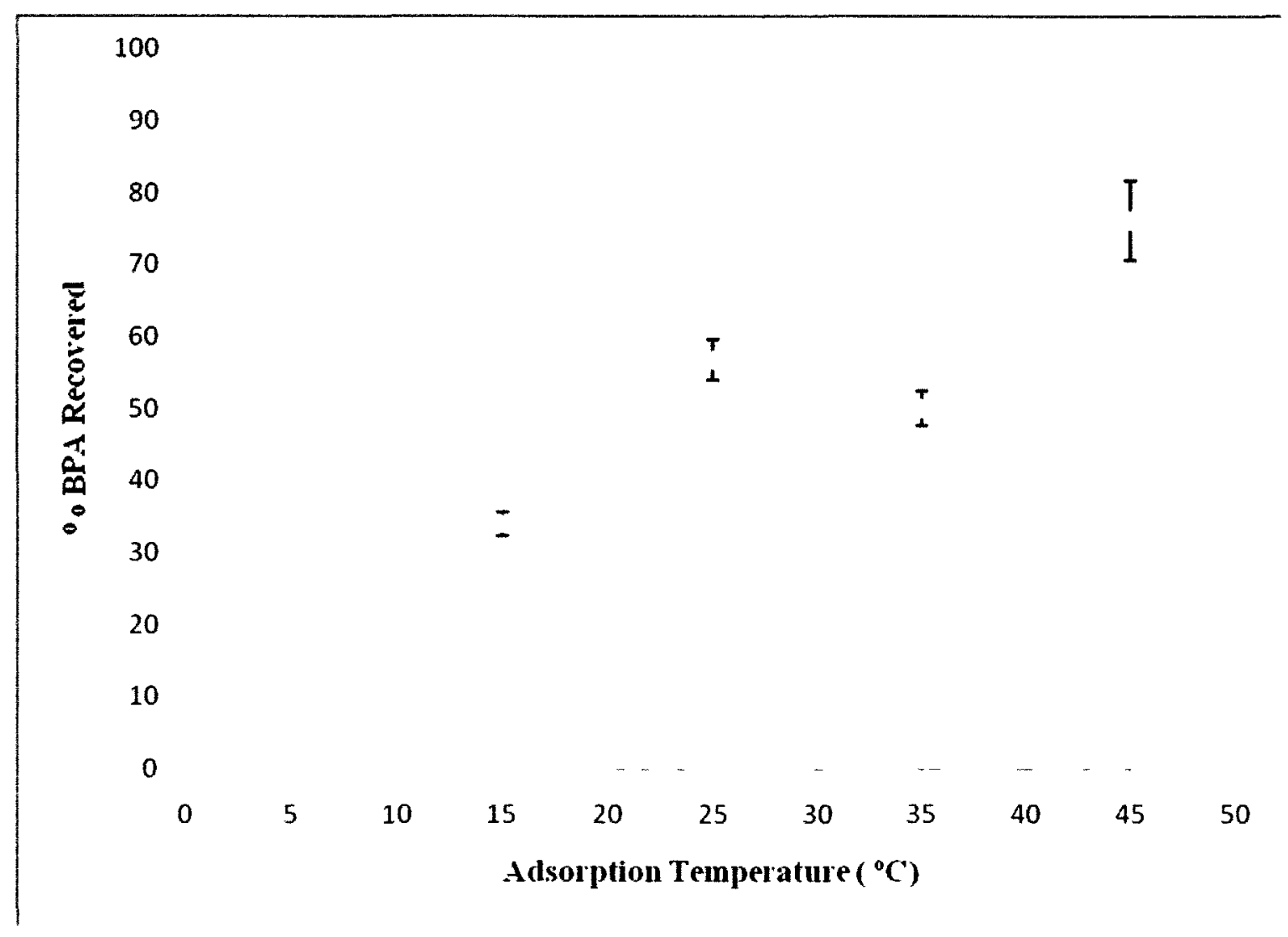

Figure 3-48 BPA recovery with changing adsorption temperature.

The thermodynamics of the adsorption process can be determined when adsorption is measured over a range of temperatures. The spontaneity of the system in standard state is modelled by the equation for Gibbs free energy:

$\Delta G^{\circ}=\Delta H^{\circ}-T \Delta S^{\circ}$

And also by the van Hoff equation:

$\Delta G^{\circ}=-R T \ln K_{e q}$ 
Where $\Delta \mathrm{G}^{\circ}$ is the standard free energy, $\Delta \mathrm{H}^{\circ}$ is the enthalpy of the process, $\Delta \mathrm{S}^{\circ}$ is the entropy of the process, $\mathrm{T}$ is the temperature and $\mathrm{K}_{\mathrm{eq}}$ is the equilibrium constant. Despite the fairly hydrophobic CNT surface compared to water, it has been shown that water also adsorbs to the surface of carbon nanotubes. In order for adsorption to occur, the waterBPA bond and water-CNT bonds must be broken to allow formation of BPA-CNT bonds. Energy is required to break the BPA- $\mathrm{H}_{2} \mathrm{O}$ and $\mathrm{H}_{2} \mathrm{O}-\mathrm{CNT}$ bonds. If the energy released upon formation of the BPA-CNT bonds is larger than the energy required to break the other bonds adsorption will be favoured. Furthermore, in solution, BPA is solvated by water molecules which is an ordered system. When BPA adsorbs to the surface of CNTs, it will also be confined on the nanotube surface. In order to understand which is more favourable, BPA- $\mathrm{H}_{2} \mathrm{O}$ or BPA-CNT interactions, the entropy change must also be calculated.

The equations shown above can be made equal as both are a function of $\Delta \mathrm{G}^{\circ}$ :

$-R T \ln K_{e q}=\Delta H^{\circ}-T \Delta S$

This in turn can be written as a function of the equilibrium constant:

$\ln K_{e q}=-\frac{\Delta H^{\circ}}{R T}+\frac{\Delta S^{\circ}}{R}$

The equilibrium constant can be calculated at each temperature and then $\ln \mathrm{K}_{\mathrm{eq}}$ can be plotted as a function of $1 / \mathrm{T}$. The slope (m) and y-intercept (b) of this plot can be used to determine the enthalpy and entropy of the system: 
$m=-\frac{\Delta H^{\circ}}{R}$ and $b=\frac{\Delta S^{\circ}}{R}$

Figure 3-49 shows the relationship between $\ln \mathrm{K}_{\mathrm{eq}}$ and $1 / \mathrm{T}$. The effect of temperature on BPA adsorption to CNTs was only studied at five temperatures which were easily accessible in the lab.

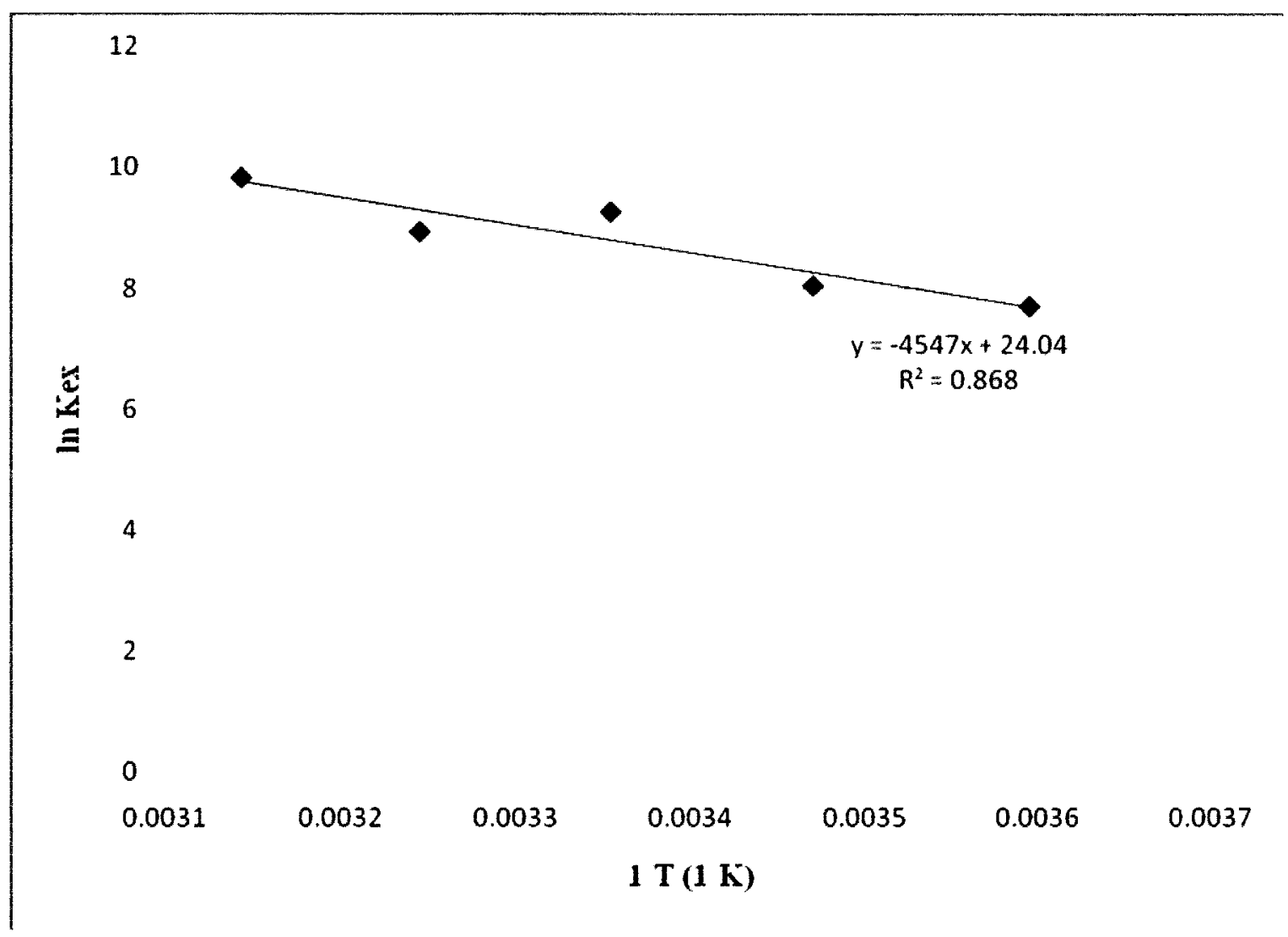

Figure 3-49 van Hoff plot showing correlation between equilibrium constant and temperature.

Using the thermodynamic equations for slope and $y$-intercept from the van Hoff plot, the enthalpy and entropy changes of the system were calculated to be $37.8 \mathrm{~kJ} / \mathrm{mol}$ and 200 $\mathrm{J} / \mathrm{K} \cdot \mathrm{mol}$, respectively. The positive $\Delta \mathrm{H}^{\circ}$ and $\Delta \mathrm{S}^{\circ}$ values imply BPA adsorption is endothermic and that BPA is less ordered on the CNT surface, however these values are 
the enthalpy and entropy changes for the overall reaction of moving BPA from water to the CNT surface. As mentioned, in order for BPA adsorption to occur, the BPA- $\mathrm{H}_{2} \mathrm{O}$ bonds and $\mathrm{H}_{2} \mathrm{O}-\mathrm{CNT}$ bonds must be broken to allow the formation of BPA-CNT bonds. If not all BPA is solvated by water in aqueous solution, BPA-BPA bonds may also exist, which will need to be overcome as well. Since adsorption does occur, the interactions between BPA and water, and water and CNTs are overcome. As previously mentioned, BPA adsorption to the CNTs can be modeled by the reaction:

$\mathrm{BPA}_{(\mathrm{aq})}+\mathrm{CNT}_{(\mathrm{aq})} \underset{k_{-1}}{\stackrel{k_{1}}{\rightleftarrows}} \mathrm{BPA}-\mathrm{CNT}$ (aq)

The enthalpy change of this reaction can be written as:

$\Delta \mathrm{H}_{(\mathrm{rxn})}^{\circ}=\Delta \mathrm{H}_{(\mathrm{BPA}-\mathrm{CNT})}^{\circ}-\left[\Delta \mathrm{H}^{\circ}{ }_{(\mathrm{BPA})}+\Delta \mathrm{H}^{\circ}{ }_{(\mathrm{CNT})}\right]$

Thus, $\Delta \mathrm{H}^{\circ}{ }_{(\mathrm{rxn})}$ has been determined here but the other variables in the equation are unknown and were not measured experimentally. Formation of a bond is always an exothermic process while energy is needed to break a bond which is thus, an endothermic process. In other words, although the heats of the reactants and product are not known for BPA adsorption, the sign of $\Delta \mathrm{H}^{\circ}{ }_{(\mathrm{BPA}-\mathrm{CNT})}$ will be negative since a bond is being formed and the signs of $\Delta \mathrm{H}^{\circ}{ }_{(\mathrm{BPA})}$ and $\Delta \mathrm{H}^{\circ}{ }_{(\mathrm{CNT})}$ will positive as bonds are being broken. Since the calculated enthalpy is positive and suggests the overall reaction is endothermic, the sum of $\Delta \mathrm{H}^{\circ}{ }_{(\mathrm{BPA})}$ and $\Delta \mathrm{H}^{\circ}{ }_{(\mathrm{CNT})}$ must be greater than the negative $\Delta \mathrm{H}^{\circ}{ }_{(\mathrm{BPA}-\mathrm{CNT})}$. Individually, the contributions from $\Delta \mathrm{H}^{\circ}{ }_{(\mathrm{BPA})}$ and $\Delta \mathrm{H}^{\circ}{ }_{(\mathrm{CNT})}$ may be moderate and when added the sum is greater than $\Delta \mathrm{H}^{\circ}{ }_{(\mathrm{BPA}-\mathrm{CNT})}$, or the energy required to break one of the interactions (BPA- $\mathrm{H}_{2} \mathrm{O}$ or $\mathrm{H}_{2} \mathrm{O}-\mathrm{CNT}$ ) may significantly outweigh the other and its 
contribution alone may be greater than $\Delta \mathrm{H}^{\circ}{ }_{(\mathrm{BPA}-\mathrm{CNT})}$. Further evidence is needed to deduce conclusions about the entropy of the system. Similarly, the entropy of the reaction can be written in the same way. The positive value of $\Delta \mathrm{S}^{\circ}$ calculated from the van't Hoff plot suggests overall BPA adsorbed to the CNTs is less ordered than when the BPA is dissolved in water and water is adsorbed to the CNT surface. More evidence about the individual entropies of the products and reactants is needed to make further conclusions about the entropy of the individual contributions of the system.

Although Basheer et al. [97] did not investigate BPA adsorption to $\mu$-SPE devices, the extraction efficiency of organophosphorous pesticides (as studied by Basheer et al.) also increased at elevated temperatures. Therefore, the nature of the adsorption system may play a role in the enthalpy and entropy changes of the system.

\subsubsection{Effect of Sample Size and Concentration}

The relationships between adsorption and sample concentration and sample volume were also explored. Since sample concentration and sample volume are related, these have been grouped together for discussion. First, the effect of varying sample concentration with constant sample volume was explored. Next, sample concentration was maintained constant while aqueous sample volume was varied to examine BPA extraction. When sample concentration is held constant while sample volume is changed, the mass of BPA present in each solution will also invariably change, proportionate to the increase or decrease in sample volume. Therefore, a third parameter, constant BPA mass in solution with changing sample volume (thus resulting in changing sample concentration) was also explored. 
To determine the effect of sample concentration at constant sample volume, several BPA in water solutions were prepared at concentrations ranging from $0.05-250 \mathrm{ng} / \mathrm{mL}$. Figure 3-50 reveals the effect of changing concentration on BPA extraction. BPA extracted from the CNT envelope was detected at levels as low as $0.5 \mathrm{ng} / \mathrm{mL}$. At lower concentrations, 0.05 and $0.1 \mathrm{ng} / \mathrm{mL}$, BPA was also detectable, however the mass balance was not $100 \%$. This was likely the result of contamination somewhere in the system during extraction and the results have been omitted from this figure. Although the lowest detectable BPA in water standard from the calibration curve was $25 \mathrm{ng} / \mathrm{mL}$, PVDF-CNT envelopes could be used to concentrate BPA from aqueous solution at concentrations 50 times lower than the instrument detection limit. As such, the limit of detection of the method is $0.5 \mathrm{ng} / \mathrm{mL}$, or $0.5 \mathrm{ppb}$. 


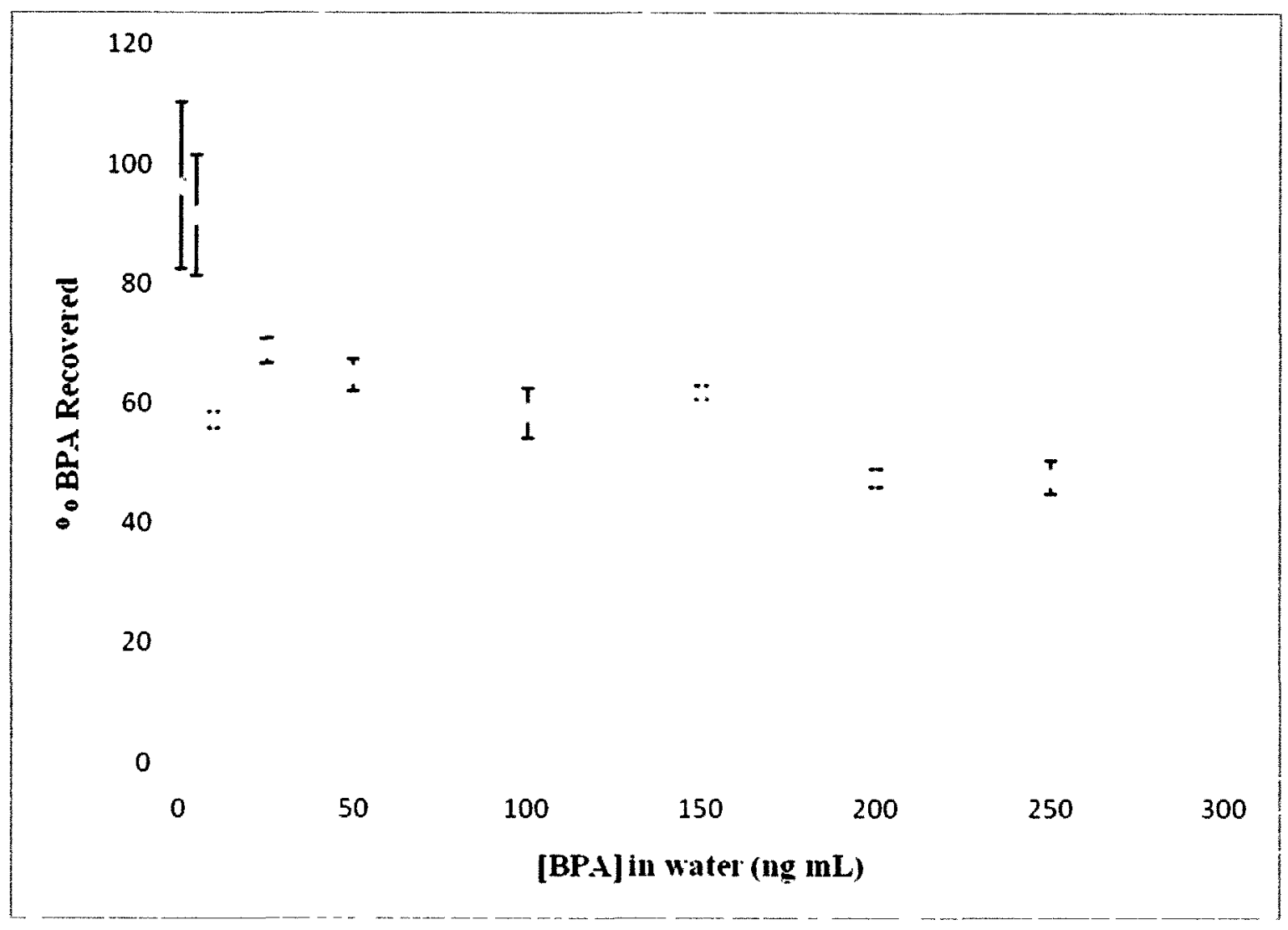

Figure 3-50 BPA extraction from aqueous solution with changing concentration at constant sample volume.

It can been seen that the amount of BPA removed remains constant as concentration is initially decreased but eventually approaches $100 \%$ at very low concentrations. Since the amount of BPA in each solution was different, the increase in extraction efficiency is most appropriately expressed by examining the equilibrium expression. To recall, BPA equilibrium is governed by the expression:

$K_{e x}=\frac{\frac{S A_{B P A, C N T}}{S A_{C N T}}}{\frac{[B P A]_{H 2 O}}{[B P A]_{H 2 O}^{0}}}$ 
Since the surface area of BPA on the CNT and the surface area of the CNTs themselves is a function of the mass of BPA adsorbed and the mass of CNTs respectively, and the concentration of BPA at standard conditions is $1 \mathrm{M}$, the equilibrium expression can be modified:

$$
K_{e x}=\frac{\frac{m_{B P A, C N T}}{m_{C N T}}}{\frac{m_{B P A, d H_{2} O}}{V_{d H_{2} O}}} \frac{1 m o l / L}{1 m}
$$

The partition coefficient at each concentration was calculated, and the relationship between concentration and partition coefficient is plotted in Figure 3-51. An increase in the value of the partition coefficient constant at very low concentrations indicates the equilibrium has shifted. At such low concentrations, the amount of BPA partitioning to the CNTs approaches the total amount of BPA in solution. At these concentrations, the PVDF-CNT envelope deviates from equilibrium extraction behaviour and the apparent equilibrium constant is larger.

During all experiments, the amount of BPA left in water was always determined in addition to the amount of BPA extracted by the PVDF-CNT envelope. Finding the amount of BPA left in water is useful for ensuring mass balances and is necessary for equilibrium constant calculations. However, this quantity could not be measured at low concentrations employed in this experiment since it was often below the instrument detection limit. Thus the concentration of BPA left in water was determined by difference from the amount of BPA extracted by the envelope, assuming a $100 \%$ mass 
balance. As a result, larger error is introduced at low initial BPA concentration but the relationship shown in Figure 3-51 is clear. The value of $\mathrm{K}_{\mathrm{ex}}$ is essentially constant and is equal to 8740 .

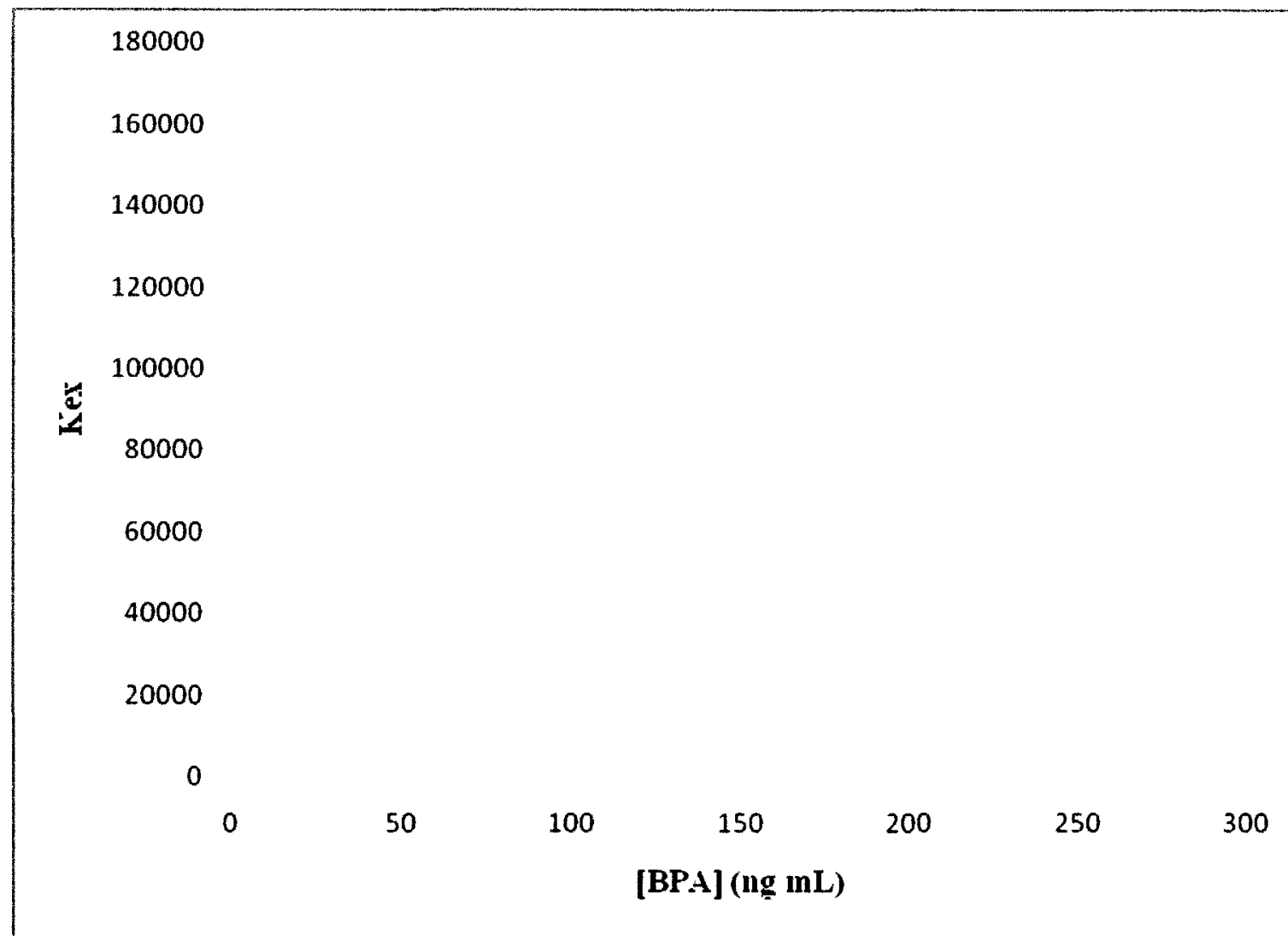

Figure 3-51 Partition coefficient as a function of initial aqueous BPA concentration using PVDF-CNT envelopes.

The effect of changing sample volume with constant concentration was also examined. In SPME, when equilibrium (not exhaustive) extraction is examined, sample size does not affect the position of the equilibrium. Thus, SPME techniques can be used for small samples in the laboratory or can be taken into the field and used for extraction directly from the media of study [73]. In other words, the SPME fiber could be dipped in a beaker of lake water or the actual lake itself and analyte extraction would eventually 
reach the same amount. Thus, to understand the effects of sample volume on BPA extraction using PVDF-CNT envelopes, constant sample concentration with changing sample volume was also examined in this work from $15-2000 \mathrm{~mL}$, as shown in Figure 352.

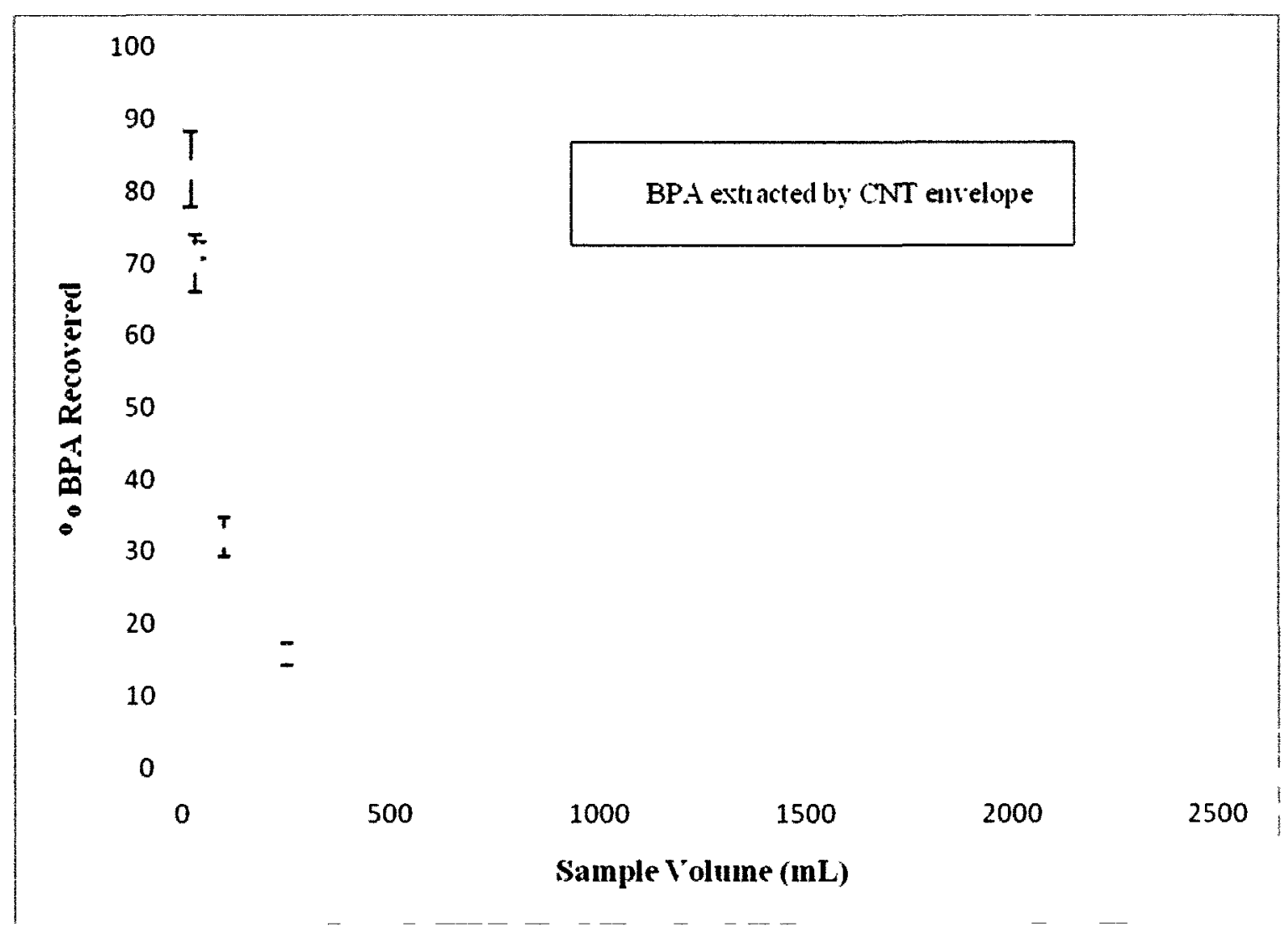

Figure 3-52 BPA extraction from aqueous solution at constant concentration with changing sample volume.

The results indicate that BPA recovery approaches $100 \%$ in a small sample size, in addition to low concentration. SPME also approaches exhaustive extraction in very small sample volumes since the distribution coefficient is so large. Considering the modified equilibrium expression shown above, BPA equilibrium can be expressed as a function of mass of BPA adsorbed to the CNTs: 
$m_{B P A, C N T}=K_{e x} \cdot m_{C N T} \cdot\left[\frac{m_{B P \sharp, H_{2}} \mathrm{O}}{V_{\mathrm{H}_{2} \mathrm{O}}}\right] \cdot 1 \mathrm{~L} / \mathrm{mol}$

The mass of BPA left in water can be expressed in terms of mass of BPA extracted since:

$m_{B P A, H_{2} O}+m_{B P A, C N T}=m_{B P A, T o t}$

And thus the equilibrium expression becomes:

$m_{B P A, C N T}=\frac{K_{e x} \cdot m_{C N T} \cdot m_{B P A, T o t} \cdot 1 L / m o l}{V_{d H_{2} O}+K_{e x} \cdot m_{C N T} \cdot 1 L / m o l}$

And the total mass of BPA can be represented by as a function of the initial concentration of BPA in aqueous solution:

$m_{B P A, C N T}=\frac{K_{e x} \cdot m_{C N T} \cdot[B P A]_{B P A, m t} \cdot V_{d H_{2} O} \cdot 1 L / m o l}{V_{d H_{2} O}+K_{e x} \cdot m_{C N T} \cdot 1 L / m o l}$

As in SPME theory $[72,73]$, when $V_{d H_{2} O} \ll K_{e x} \cdot m_{C N T} \bullet 1 L / m o l$, the equilibrium expression can be simplified to:

$m_{B P A, C N T}=[B P A]_{B P A, m z} \cdot V_{d H_{2} O}$

which is identical to the SPME expression for exhaustive extraction.

The equilibrium coefficient of each sample size can again be calculated to observe this relationship. Figure 3-53 depicts the relationship between sample size and partition coefficient and confirms that the coefficient increases with decreased sample size. 


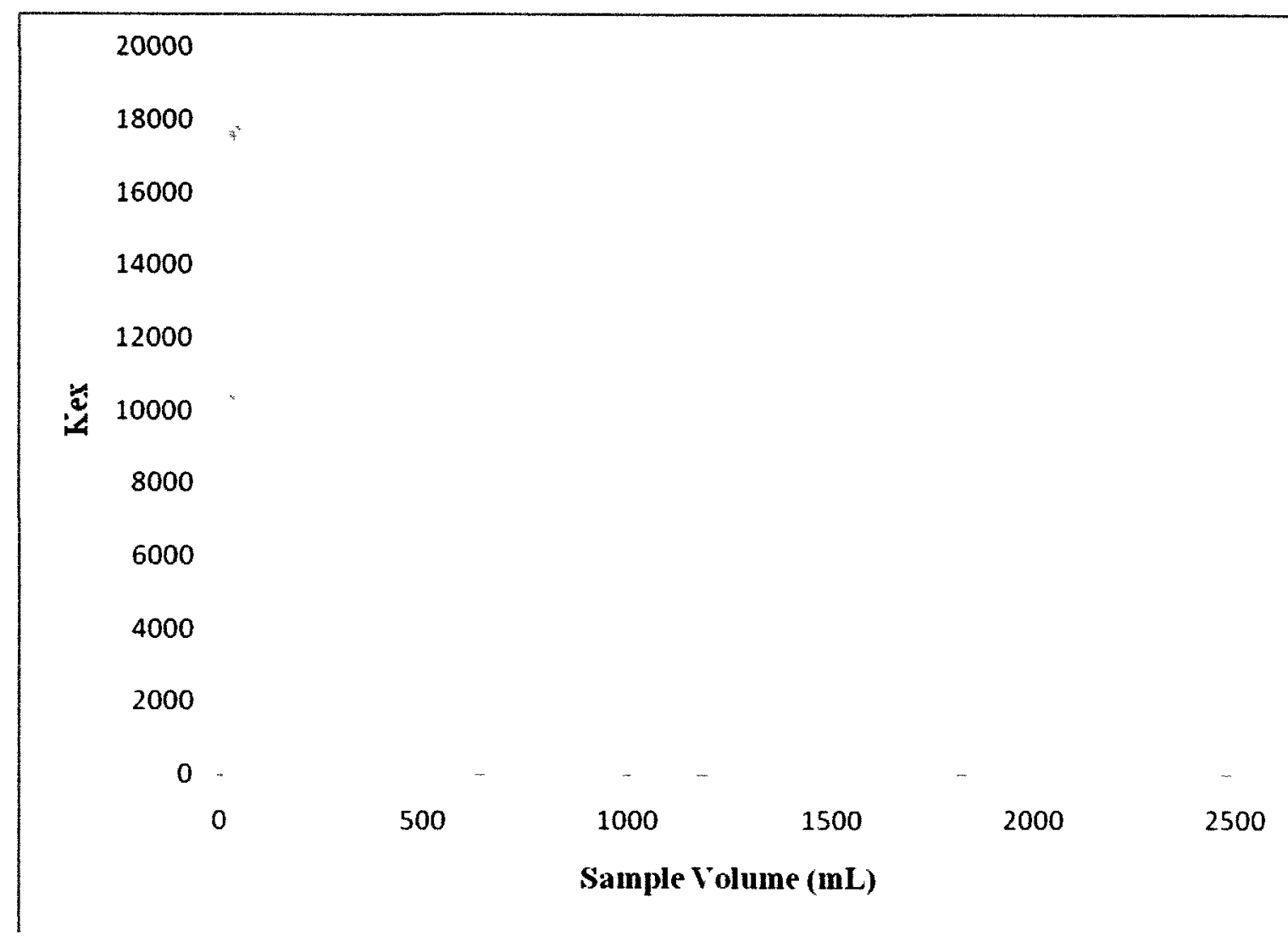

Figure 3-53 Relationship between partition coefficient and sample volume.

The effect of sample volume was also explored with constant BPA mass. Although this relationship does not model any practical extraction situations it was explored to further understand the effect of adsorption sample volume. Thus, extractions were performed in sample sizes ranging from $16-2000 \mathrm{~mL}$ in which each solution contained $10 \mu \mathrm{g}$ of BPA. Figure 3-54 illustrates the obtained results. Likewise to the study of BPA adsorption in changing sample solution with constant concentration, the amount of BPA extracted increased with decreasing sample volume. Both of these studies indicate extractions should be performed in the smallest sample size possible if exhaustive extraction is the goal, otherwise the position of the equilibrium will not change regardless of sample size (provided sufficient equilibration time is given in larger sample volumes). 


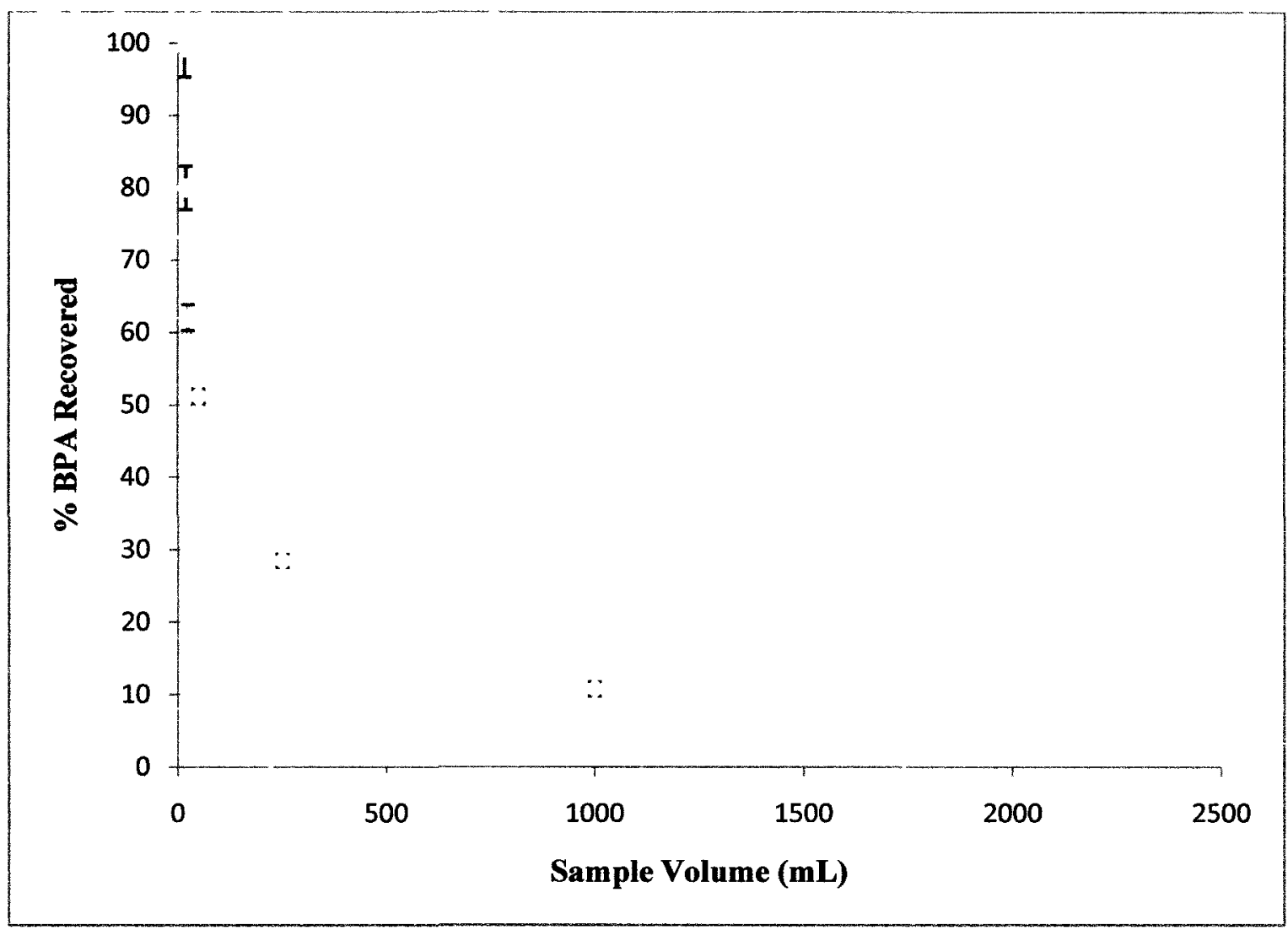

Figure 3-54 BPA extraction from aqueous solution containing the same mass of BPA with changing sample volume.

\subsubsection{Effect Mass of Carbon Nanotubes}

Several parameters were examined to optimize the carbon nanotubes inside the envelope for BPA extraction from aqueous solution. First, the minimum CNT mass needed for adsorption was explored. Several PVDF-CNT envelopes were prepared with varying mass of multi-walled nanotubes, ranging from $0.5-13.3 \mathrm{mg}$ CNTs. Attempts were made to prepare envelopes with greater masses of CNTs however as CNT mass increased it became increasingly more difficult to seal the membranes together and the effects of static were also enhanced. The outcome of this experiment is shown in Figure 3-55. Extraction equilibrium was obtained when the PVDF-CNT envelope was filled with more 
than $2 \mathrm{mg}$ of CNTs. Since the CNT surface area available for adsorption greatly exceeded the surface area of BPA adsorbed to the CNTs when only $\sim 6.45 \mathrm{mg}$ CNTs were used, the CNT envelope should not need to be filled with a higher mass of CNTs.

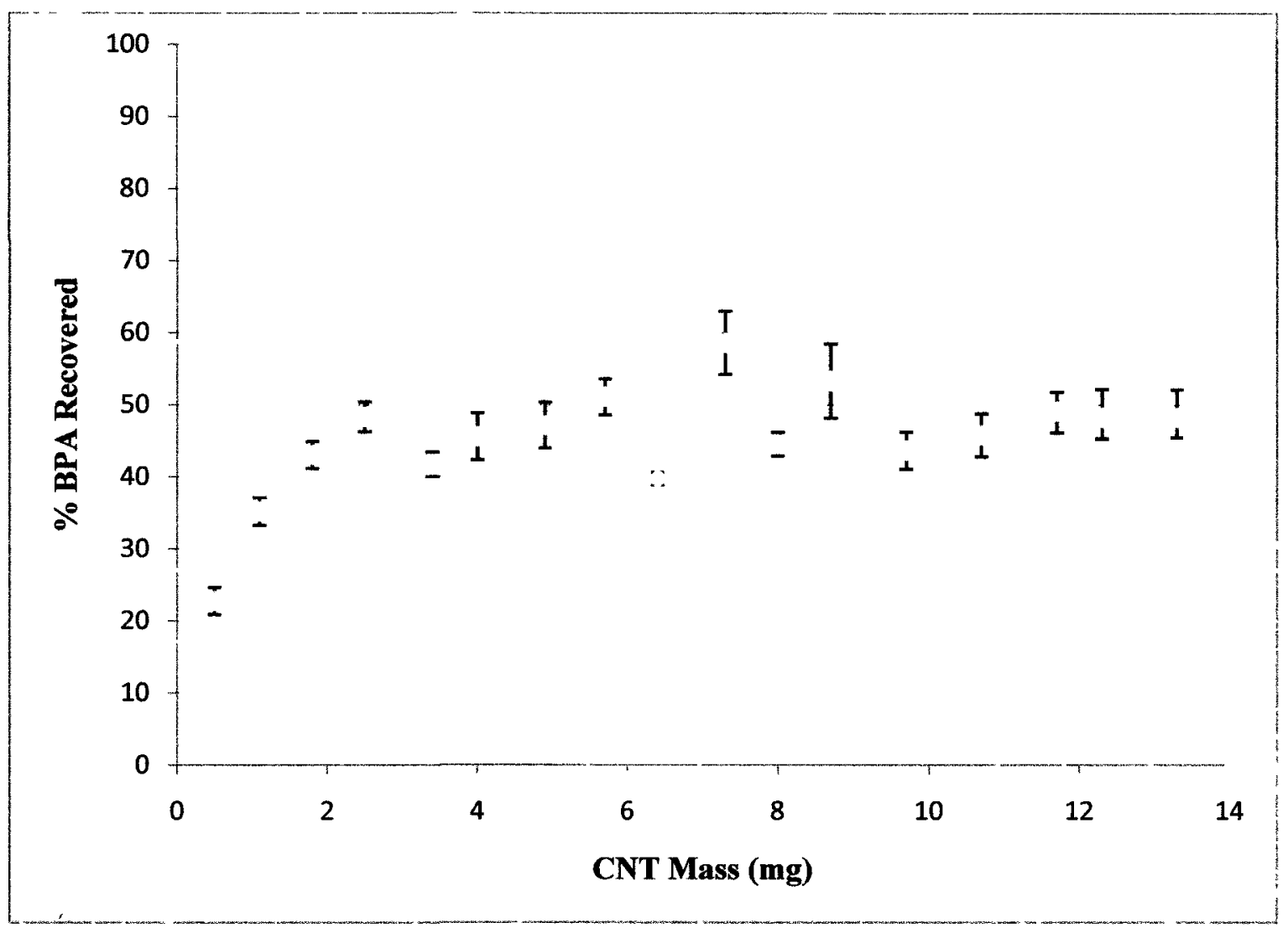

Figure 3-55 BPA extraction using PVDF-CNT envelopes filled with varying masses of LMWCNTs.

\subsubsection{Effect of Carbon Nanotube Functionality and Dimension}

In all experiments discussed thus far, the CNT envelopes were filled with pristine "long" MWCNTs (L-MWCNTs) with a diameter of $10 \mathrm{~nm}$. To determine the effect of CNT dimensions on BPA extraction, envelopes were filled CNTs of different lengths and 
diameters then those of the L-MWCNTs. Furthermore, extraction efficiency of pristine versus oxidized CNTs was also explored.

Single and multi-walled CNTs were oxidized separately in dilute nitric acid to functionalize the tubes with oxygen-containing functional groups. PVDF-CNT envelopes were filled with $\sim 6.45 \mathrm{mg}$ of either pristine single, double or multi-walled or oxidized single or multi-walled CNTs and were used for BPA extraction as depicted in Figure 356. Although the presence of an aromatic carboxylic acid or alcohol was not confirmed by IR spectroscopy for either the oxidized SWCNTs or MWCNTs, preparation of aqueous suspensions of the oxidized CNTs indicated otherwise. Water has been shown to wet the surface of pristine CNTs [106] and its capability of adsorbing to oxidized CNTs would be considerably greater since the functionalities of each would be more similar and more closely follow the principle "like dissolves like". If the oxygencontaining functionalities are primarily solvated with water molecules, BPA adsorption to these sites would not be as likely. Figure 3-56 indicates BPA recovery decreased significantly when the CNT envelope was filled with oxidized CNTs.

This figure illustrates two trends, a comparison between BPA extraction using pristine and functionalized CNTs, and also the effect of nanotube diameter on BPA recovery. Generally, CNT surface area increases with smaller tube diameter. As depicted in Figure 3-56 BPA extraction was greatest when the CNT device was filled with SWCNTs, with nanotube diameter $<2 \mathrm{~nm}$. Extraction efficiency decreased with larger tube diameter. 


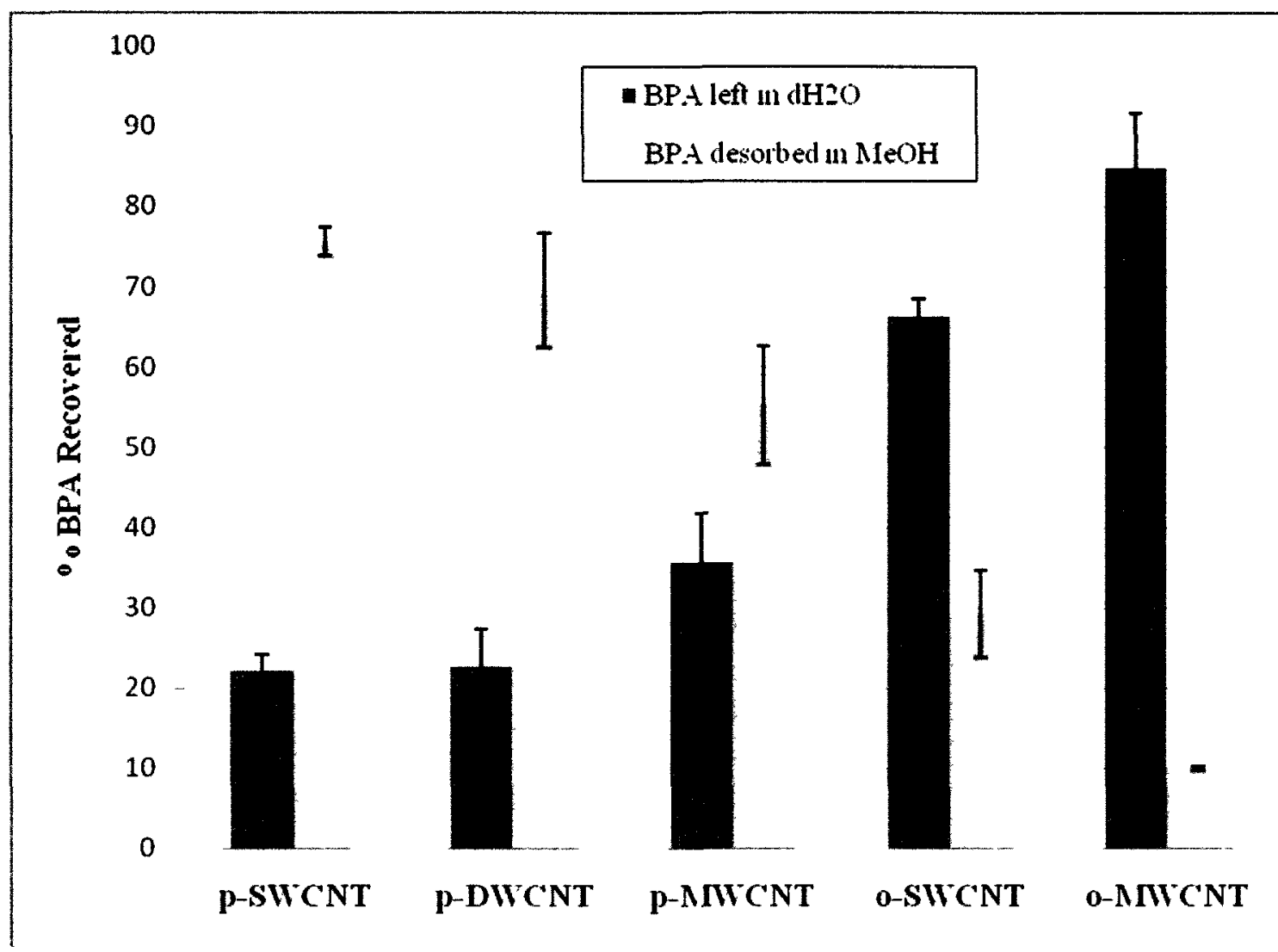

Figure 3-56 Comparison of BPA extraction using CNTs of different diameters (pristine single, double or multi-walled) and of different functionality (pristine or oxidized SWCNTs and MWCNTs)

Pan et al. [107] hypothesized possible BPA adsorption sites on the surface of CNTs. Adsorption to the surface, groove, and interstitial spaces between CNTs was examined. It was observed that adsorption of BPA to the surface of a CNT parallel to the tube axis was least favourable as the steric energy of conformation needed to keep the two benzene rings of BPA in the same plane was very high. BPA adsorption could also occur around the circumference of the CNT as BPA has a "butterfly" structure, depending on the diameter of the CNTs. Adsorption around a CNT increased with smaller tube diameter as less steric energy was required to change the molecular conformation of BPA.

Furthermore, greater BPA adsorption was observed in the groove between two CNTs in a 
bundle with smaller diameter since the angle between the two benzene rings was smaller. Adsorption only increased using larger diameter tubes in the interstitial spaces between CNTs in a bundle, since the spaces between smaller diameter CNTs were too small to accommodate BPA [107].

The total carbon nanotube surface area available for BPA adsorption in this study significantly exceeded the surface area covered by BPA molecules. Although the available surface area increased when the CNT envelope was filled with smaller diameter tubes, it was likely the increased curvature of the smaller CNTs that afforded greater BPA recovery. The MWCNTs used throughout most of the experiments were $10 \mathrm{~nm}$ in diameter, which is more than 14 times greater than the length of a BPA molecule $(\sim 700$ $\mathrm{pm})$. When the diameter of the nanotubes is that much larger than length of BPA, adsorption is not a reflection of the curvature of the nanotube since the BPA molecule essentially lays flat against the CNT surface. However, when the difference in CNT diameter and BPA length is not as significant, the curvature of the CNTs will affect BPA adsorption. The center carbon atom in BPA is $\mathrm{sp}^{3}$ hybridized and thus the phenol rings are at an angle of $109.5^{\circ}$ from one another. The SWCNTs used in this study were $<2 \mathrm{~nm}$ in diameter which is only about 3 times greater than the length of BPA. At this small diameter, BPA likely wrapped around the nanotube to adsorb to the CNT surface, which is a more favourable conformation of the molecule and likely resulted in higher extraction efficiencies.

Since BPA adsorption has been shown to be more dependent on nanotube diameter rather than length, BPA extraction using CNTs with different lengths should not 
significantly impact BPA recovery. A comparison of BPA recovery to CNTs length is shown below in Figure 3-57. CNT envelopes were filled with short MWCNTs (length: 1-2 $\mu \mathrm{m}$ ) or long MWCNTs (length: 5-15 $\mu \mathrm{m}$ ), both with an outer diameter of 10-20 nm. The extraction efficiencies were not statistically different, confirming that BPA adsorption is not a function of CNT length.

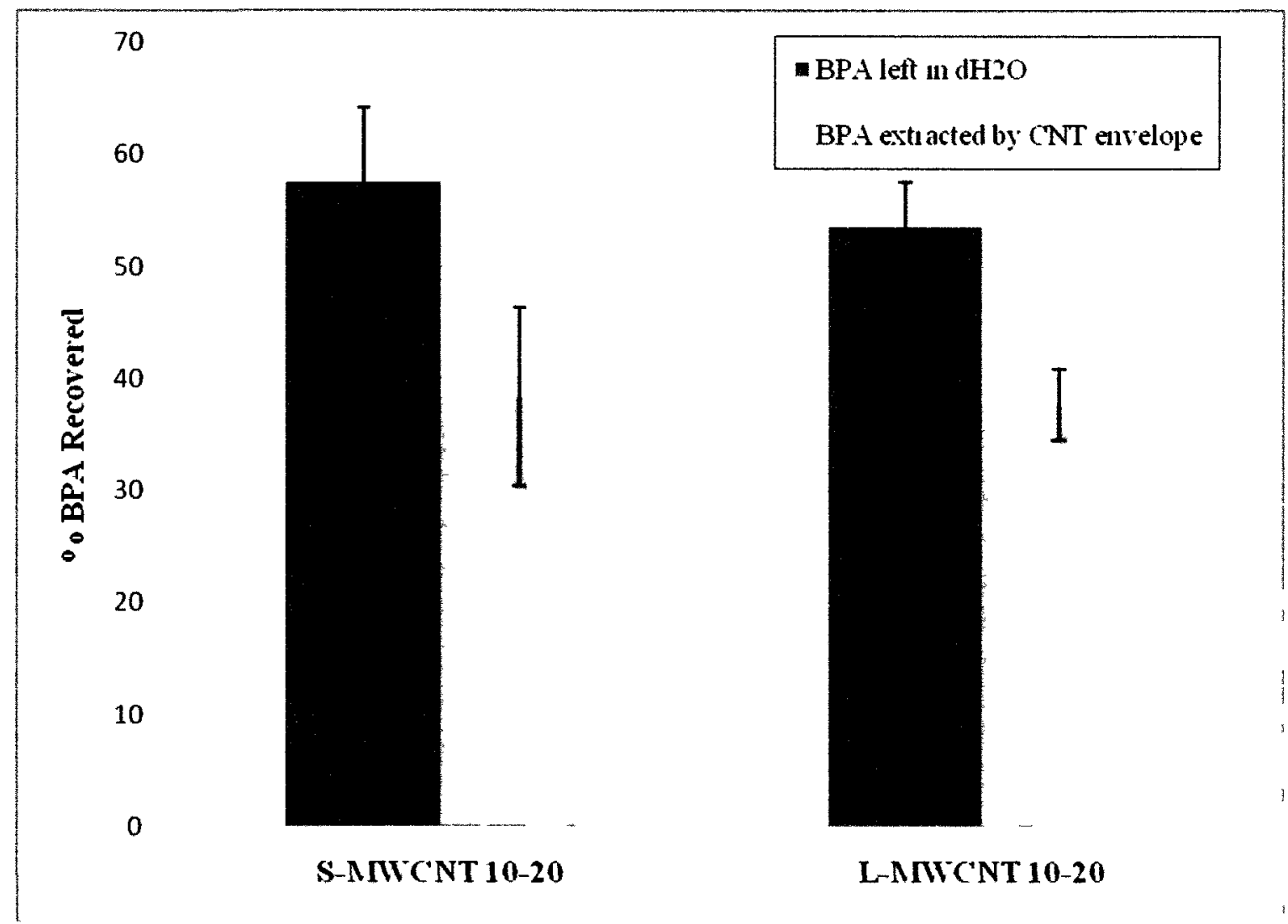

Figure 3-57 Effect of carbon nanotube length on BPA recovery when the nanotube diameter is constant.

\subsubsection{Effect of $\mathrm{pH}$}

The effect of $\mathrm{pH}$ of the aqueous BPA solution was also studied over a broad $\mathrm{pH}$ range, $\mathrm{pH}$ 2.01-12.63, to span the average of both of the pKa's for BPA. Several BPA solutions 
of the same concentration were prepared and the $\mathrm{pH}$ of each was altered by adding a few drops of dilute $\mathrm{HCl}$ or $\mathrm{NaOH}$. The $\mathrm{pH}$ of each solution was measured with a $\mathrm{pH}$ meter. Figure 3-58 indicates the effect of $\mathrm{pH}$ on BPA adsorption to CNTs. It appears from this figure than BPA recovery stays constant from $\mathrm{pH}$ 2.01-7.68 and decreases above this $\mathrm{pH}$ range, as seen at $\mathrm{pH} 10.18$ and $\mathrm{pH} 12.63$. The discrepancies in \% BPA Recovered from pH 2.01-7.68 likely just reflects experimental error from one CNT envelope to the next. BPA is fully protonated over this range and its solubility in aqueous solutions with $\mathrm{pH}$ 2.01 and $\mathrm{pH} 7.68$ were shown to be essentially equal. BPA is more soluble in aqueous solution above the first $\mathrm{pKa}(9.59)$ since one of the $-\mathrm{OH}$ groups is largely deprotonated and even more soluble still above the second $\mathrm{pKa}(11.30)$ when both $-\mathrm{OH}$ groups are deprotonated. Although the solubility of BPA in aqueous solution was only measured experimentally above the second $\mathrm{pKa}(\mathrm{at} \mathrm{pH} 12.63)$ in this work, the trend observed confirms the solubility between the first and second $\mathrm{pKa}$ would be between the solubilities measured at $\mathrm{pH} 7.68$ and $\mathrm{pH} 12.63$. As mentioned, during adsorption, interactions exist between BPA, water and CNTs. BPA- $\mathrm{H}_{2} \mathrm{O}$ bonds must be broken and water must be removed from the surface of the nanotubes in order for BPA adsorption to occur. At basic $\mathrm{pHs}$ above the first and second $\mathrm{pKa}$, the $\mathrm{BPA}-\mathrm{H}_{2} \mathrm{O}$ bonds will be considerably harder to break as the ion-dipole interactions between BPA and water are that much stronger than the dipole-dipole forces between protonated BPA and water. The strength of these interactions indicates BPA adsorption to the surface of the CNTs will be lower since deprotonated BPA is more soluble in water. 


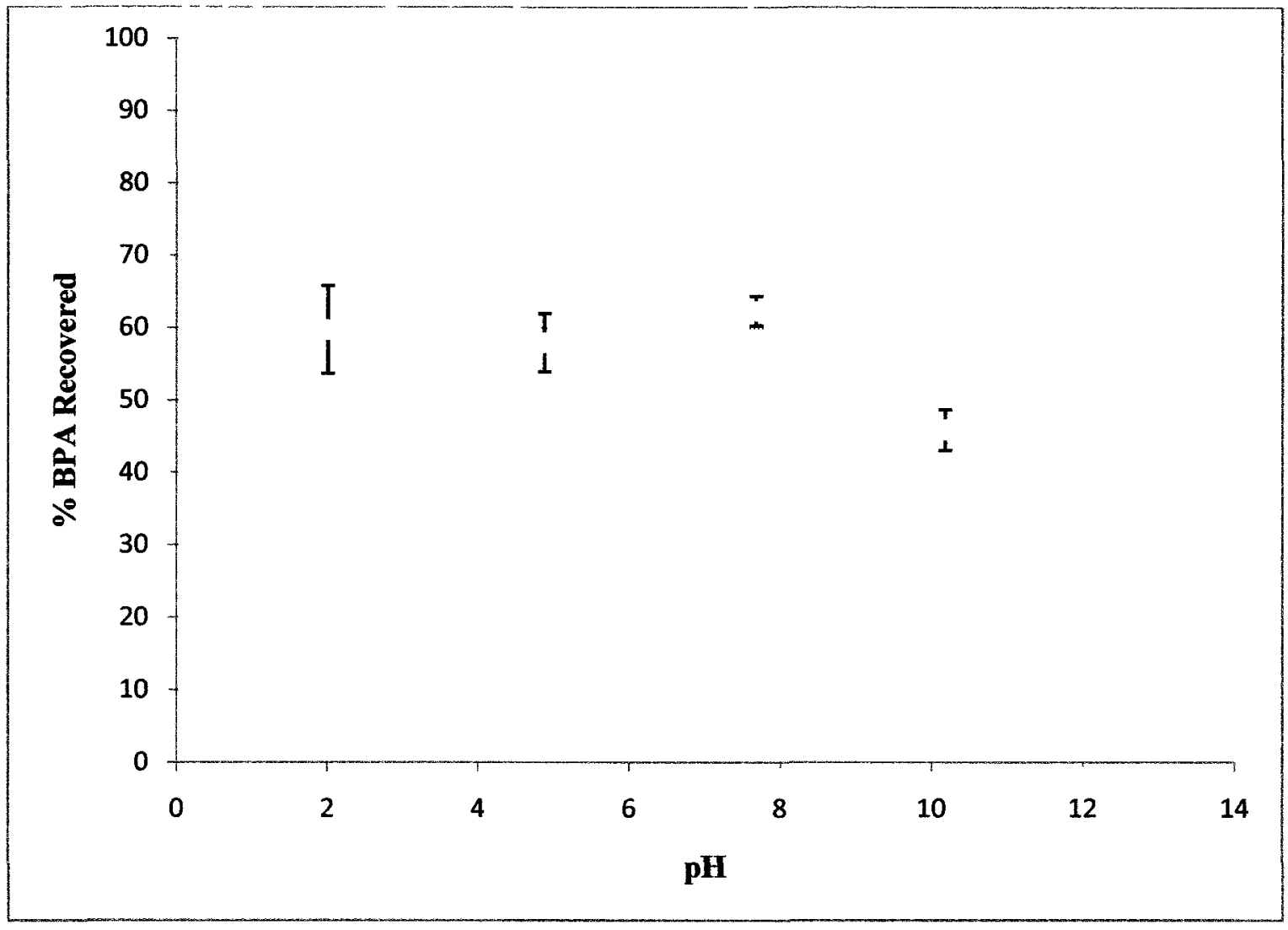

Figure 3-58 Effect of $\mathrm{pH}$ of aqueous solution on BPA extraction using PVDF-CNT envelopes.

This trend is in agreement with other works which examined BPA adsorption to carbon nanotubes. Cai et al. [83] reported no observable difference in BPA adsorption to

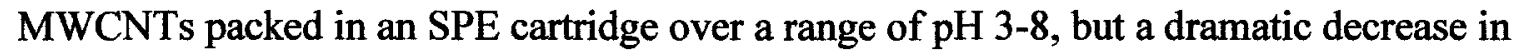
extraction above $\mathrm{pH} 8$.

\subsection{BPA Desorption from PVDF-CNT envelopes}

Similar to the adsorption process, relationships exist between the analyte, adsorbent and desorbing solvent during desorption. The interactions between the analyte and solvent, and adsorbent and solvent must be strong enough to overcome the interaction between 
the analyte and adsorbent. Desorption interactions are based on the principle of "like dissolves like". If the polarities of the analyte and solvent are similar and stronger than the "bond" formed between the analyte and adsorbent, the solvent will replace the analyte on the adsorbent and the analyte will be successfully removed. A chemical reaction can again be used to model the relationship between the components in a desorption system as shown below:

Analyte $_{(\text {adsorbed })}+$ solvent $\rightleftarrows$ Analyte $_{(\text {solvent) }}+$ Solvent $_{(\text {adsorbed })}$

In this reaction, the adsorbed analyte, the product in the adsorption reaction, is now the reactant and the interaction between analyte and adsorbent is broken to produce analyte dissolved in solvent and adsorbent dissolved in solvent. The desorption system in a PVDF-CNT envelope can be modelled by this reaction. Since methanol was used for BPA desorption in almost all experiments it is shown here as the desorption solvent. Although the CNTs do not dissolve in methanol, a strong interaction exists between them, and consequently methanol will wet the surface of the CNTs. Thus, CNTs are written as dissolved in the solvent phase.

$\mathrm{BPA}_{(\text {adsorbed })}+\mathrm{MeOH} \rightleftarrows \mathrm{BPA}_{(\mathrm{MeOH})}+\mathrm{MeOH}_{(\text {adsorbed })}$

After the initial extraction experiment was performed with the first made PVDF-CNT envelopes, analyte carryover was examined by repeating the initial extraction but desorbing the envelope twice, in two aliquots of methanol. The results from the second desorption indicated that very little BPA remained in the envelope after the first desorption which was attributed to the high solubility of BPA in methanol. Furthermore, 
mass balances between the \% BPA left in water and the \% BPA extracted by the envelope indicated very little BPA is left on the carbon nanotubes. Thus, the equilibrium in the chemical reaction of BPA desorption from a $\mathrm{CNT}$ envelope lies far to the right since essentially all BPA dissolves in methanol in this system.

\subsubsection{Desorption Rate}

In addition to determining the minimum time required for adsorption, the minimum time necessary for desorption was also explored. The BPA desorption rate was studied by varying the desorption time over several extractions while all other extraction parameters were held constant. The results of this experiment are shown in Figure 3-59. It can be seen that the desorption curve began to plateau after 5-10 minutes. BPA is extremely soluble in methanol and methanol has a strong ability to wet the carbon nanotubes, so it is not surprising that the desorption equilibrium was reached so quickly. When the desorption of organophosphorous pesticides from sewage sludge samples was examined using the $\mu$-SPE device [97], 30 minutes was determined to be the optimal desorption time for all pesticides. The desorption time for experiments using PVDF-CNT envelopes was set at 60 minutes to ensure all BPA was removed from the envelope which is comparable to the study of desorption rate reported by Basheer et al. [97]. The rate at which the analytes are eluted from the $\mu$-SPE device or CNT envelope will be largely dependent on the compatibility of the analyte and desorption solvent, and the ability of the desorption solvent to adsorb to the surface of the CNTs. 


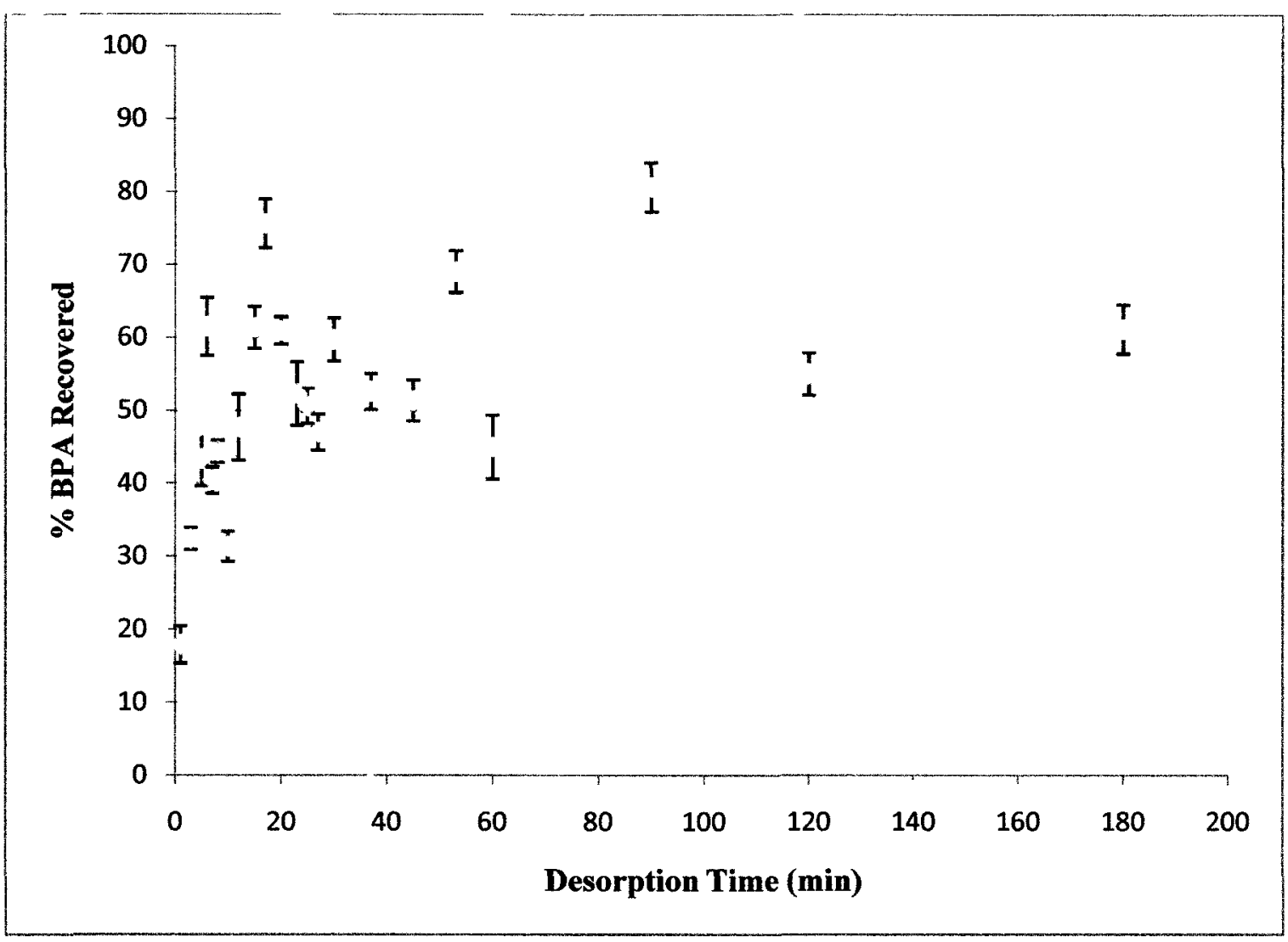

Figure 3-59 BPA desorption from PVDF-CNT envelopes with stirring.

Desorption rate was also examined without stirring and almost identical results were obtained compared to the use of stirring, as shown in Figure 3-60. An equilibrium was established after the PVDF-CNT envelopes were immersed in methanol for only 5-10 minutes, as was observed with the use of stirring. Therefore the use of agitation was not needed to break the BPA-CNT bond and drive the desorption rate to equilibrium. In SPME, the fiber often needs to be heated in order to promote desorption $[69,70]$. To be prudent, it was decided the time required for complete desorption from the envelope without stirring should be 120 minutes, for reasons discussed below. Although the pore size of the membrane was determined to be the limiting factor contributing to the lengthy 
adsorption rate, diffusion through the pores did not affect the rate of BPA desorption from the envelope.

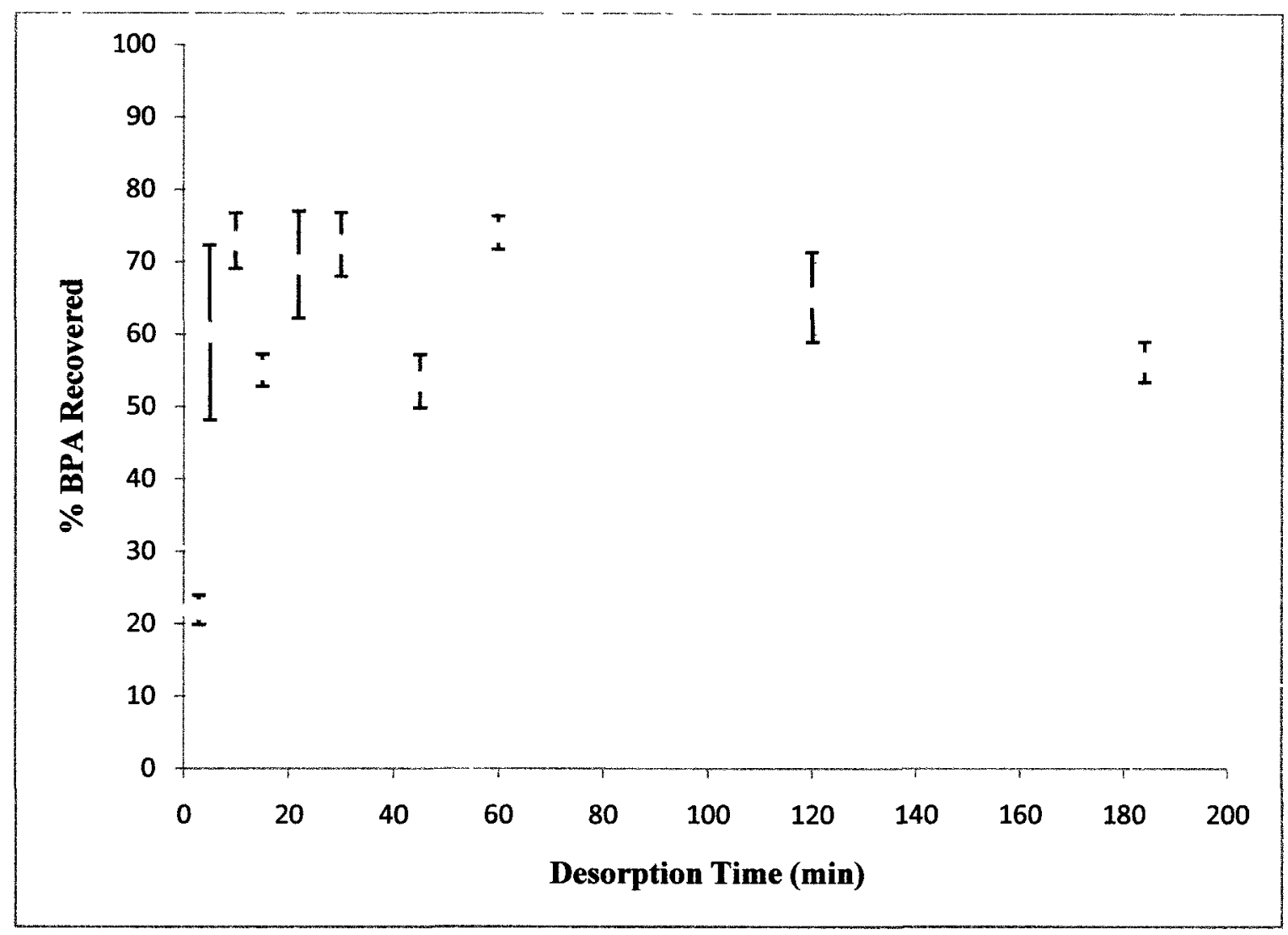

Figure 3-60 BPA desoprtion from PVDF-CNT envelopes without stirring.

\subsubsection{Desorption Solvent Volume}

Trace components in a mixture are often present at concentrations below the detection limit. Sample preparation techniques, including solid-phase extraction, can be utilized to preconcentrate the analytes prior to analysis so they are in a detectable range. The degree of concentration can be described by a pre-concentration factor, which is the ratio of sample volume to volume of eluting solvent, as shown below:

Pre-concentration factor $=$ Sample volume $/$ Desorption volume 
If the pre-concentration factor of a given sample preparation method is high, then analysis of the components at lower concentrations can be performed, which is ideal. In addition, a high pre-concentration factor likely indicates that small volumes of harmful organic solvents are being used to concentrate analytes from large sample volumes, which is desirable compared to the volumes of solvent used in liquid-liquid extraction procedures. In this study, the pre-concentration factor was determined once the minimum volume of desorption solvent needed was found.

After the minimum time required for desorption was established, the minimum volume of solvent necessary for complete desorption was also explored. The minimum volume of methanol needed was first explored with stirring over a range of $15-40 \mathrm{~mL}$, as displayed in Figure 3-61. No change in extraction efficiency was observed as the volume of methanol used was decreased. A sample volume of $40 \mathrm{~mL}$ was maintained throughout all PVDF-CNT envelope investigations, except during the studies of varying sample volume. This translates to a pre-concentration factor of only $\sim 2.67$ when $15 \mathrm{~mL}$ of methanol were used to elute BPA from the envelope. This pre-concentration factor is quite low and could be improved significantly if smaller volumes of methanol were accessible. The concentrations used in this study are several orders of magnitude lower than the maximum solubility of BPA in solution and thus very small volumes of solvent in the $\mathrm{mL}$ range should be more than capable of removing BPA from the envelope without analyte loss. 


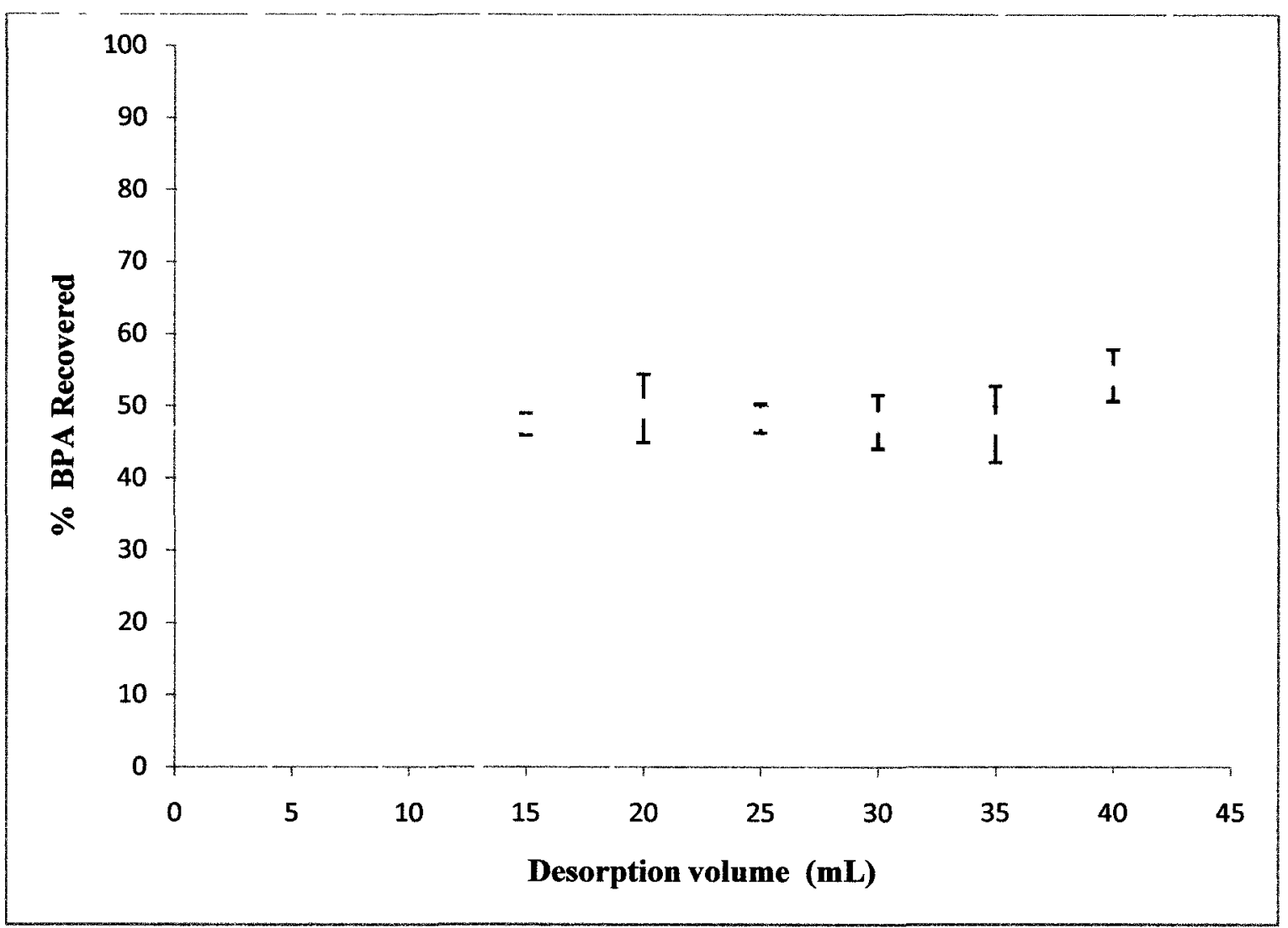

Figure 3-61 Volume of methanol required for complete BPA desorption with stirring.

The physical presence of the stir bar prevented the PVDF-CNT envelope from being suspended any lower in the vial and consequently $15 \mathrm{~mL}$ was smallest volume of methanol that could be used to completely immerse the envelope. However, without stirring, the envelope could lie flat on the bottom of the glass vial and be submerged in significantly smaller volumes of solvent, as shown in Figure 3-62. A stir bar was placed on top of the envelope to prevent it from floating, and thus keep it completely covered in solvent. 


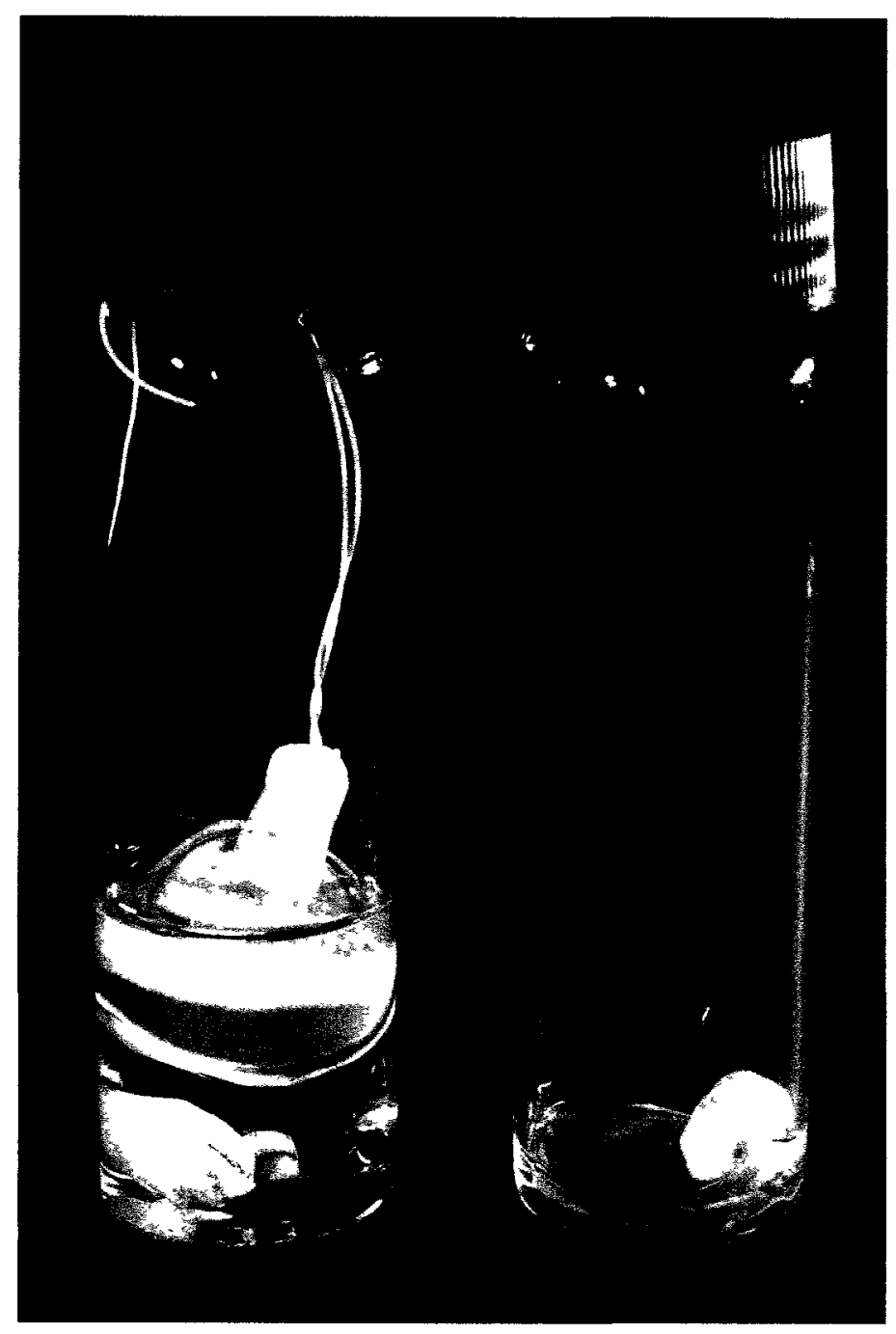

Figure 3-62 Two methods of suspending PVDF-CNT envelopes: on left, a PVDF-CNT envelope suspended in $15 \mathrm{~mL}$ methanol and on right, a PVDF-CNTenvelope could be suspended in $1 \mathrm{~mL}$ of methanol.

The curve displaying BPA extraction using varying volumes of desorption solvent without stirring is shown in Figure 3-63. Without stirring, a volume as low as $1 \mathrm{~mL}$ of methanol could be studied for BPA desorption. The exposed surface of the envelope was only just covered when $1 \mathrm{~mL}$ methanol was used, however the extraction efficiency was not compromised. To ensure all BPA was extracted from the envelope with such a small volume of solvent, a generously long desorption time of 2 hours was employed, as 
mentioned in the discussion of desorption rate without stirring. By eliminating stirring from the desorption system, pre-concentration of BPA from aqueous solution was significantly improved as the adjusted pre-concentration factor was increased to 40 . The improvement was actually so substantial that the concentration of BPA in methanol after desorption produced an HPLC signal that was too concentrated to be integrated by the instrument. The concentration of BPA in the aqueous sample was maintained at 250 $\mathrm{ng} / \mathrm{mL}$ for most analyses, which is not representative of trace concentrations in the environment. However, when the effect of concentration with constant sample volume was explored, the PVDF-CNT envelope could be used extract BPA at concentrations below its detection limit in water because the BPA on the CNTs could be concentrated in only $1 \mathrm{~mL}$ of methanol. 


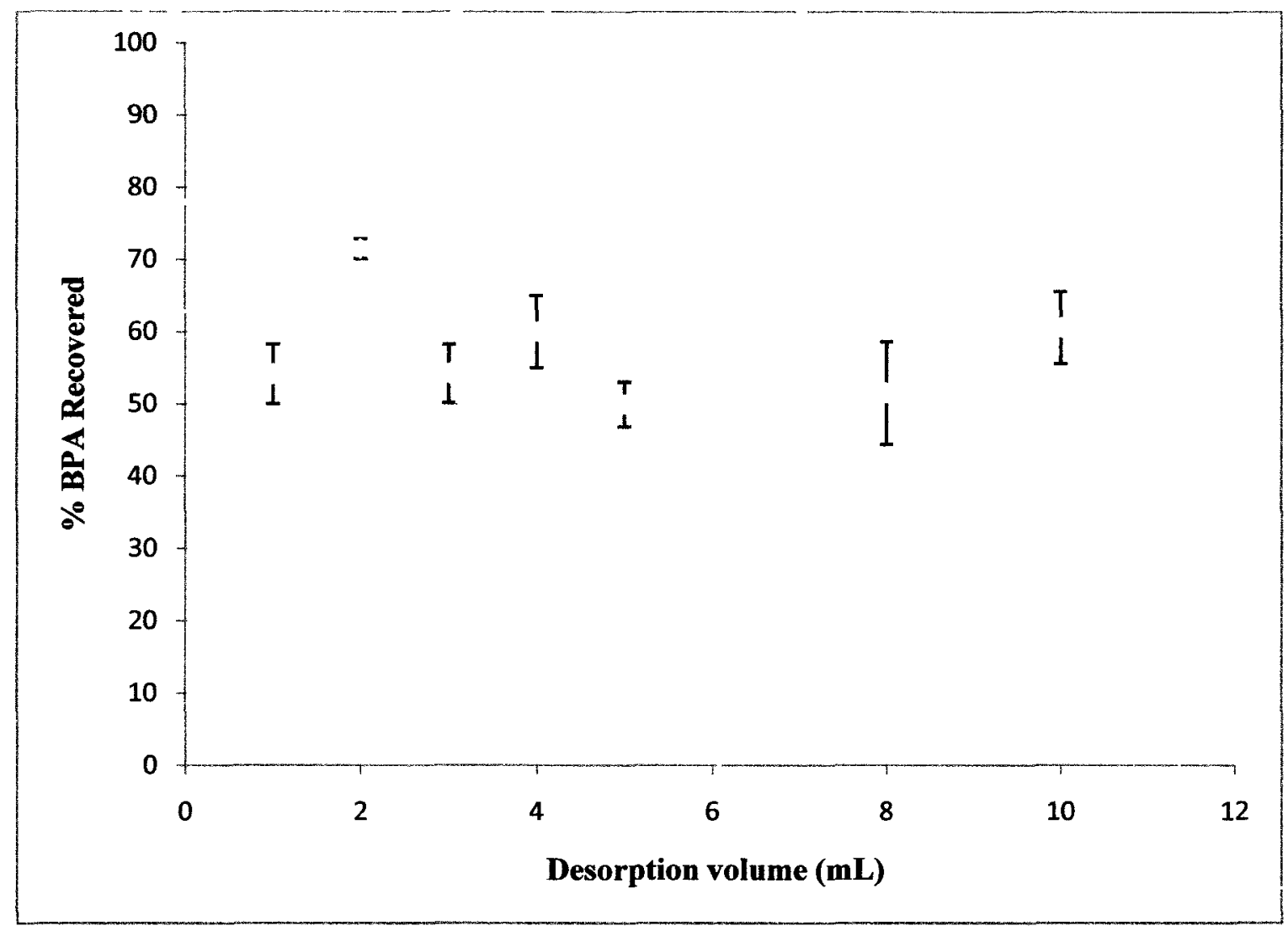

Figure 3-63 Volume of methanol required for complete BPA desorption without stirring.

When sample preparation techniques are evaluated based on the use of organic solvents, solid-phase extraction methods are favourable compared to liquid-liquid extraction procedures since smaller solvent volumes are required. In particular, SPME is an advantageous choice for pre-concentration since little to no solvent is required for analyte desorption. If the fiber is directly attached to the chromatographic system, no separate desorption step is necessary, or if desorption is performed first, minute quantities of solvent are suitable. Like SPME, analysis using PVDF-CNT envelopes can be performed with insignificant quantities of methanol. 
Basheer et al [97] reported an inconceivable approach to desorb organophosphorous pesticides from the $\mu$-SPE device. The dimensions of the device were reported to be 2 $\mathrm{cm} \times 1.5 \mathrm{~cm}$. During desorption, the device was placed in a $100 \mu \mathrm{L}$ autosampler vial and immersed in $100 \mu \mathrm{L}$ of organic solvent. Adsorption was performed in $20 \mathrm{~mL}$ of sewage sludge for most experiments, and thus the preconcentration factor for this procedure would have been tremendous. However, the fragility of the polypropylene membrane makes it difficult to roll or compress the device without ripping the membrane. It is challenging to understand how the device with these reported dimensions could have been compacted to fit in $100 \mu \mathrm{L}$ of organic solvent.

\subsubsection{Desorption solvent}

It has been shown that BPA is extremely soluble in moderately polar solvents. Methanol was initially chosen for BPA desorption from CNT envelopes as BPA is so soluble in it and methanol was a major component of the mobile phase for HPLC analysis. When the maximum solubility of BPA in common organic solvents was explored, it was determined that BPA is equally soluble in methanol and acetone, and almost as soluble in ethyl acetate and acetonitrile. However, the concentration of BPA in all of these solvents is several orders of magnitude greater than the concentrations studied for BPA extraction using CNT envelopes and thus the desorption capability of all of these solvents should be fairly equal. To test this hypothesis, extractions were performed and desorption was carried out in methanol, acetone or acetonitrile. Ethyl acetate and water are not miscible and since water is a component of the mobile phase ethyl acetate was excluded from the test of most appropriate desorption solvent. To ensure the envelope was in contact with 
the solvent, BPA desorption from the envelopes was performed in $15 \mathrm{~mL}$ of solvent. Desorption extracts were analyzed by HPLC along with BPA standards that were prepared in the given solvent. This way, the $\%$ BPA recovered would be more accurate since the samples were compared against standards prepared in the same solvent.

Figure 3-64 below displays the BPA extraction efficiencies when desorption was performed in these solvents. It can be seen that BPA desorption is higher in methanol compared to acetonitrile. As mentioned, the solubility of BPA in methanol is slightly higher than acetonitrile and thus it could be hypothesized methanol would have a greater ability to elute BPA from CNT envelopes. However, only the interactions between analyte and solvent were examined when the solubility of BPA in organic solvents was examined. The presence of the sorbent complicates the system since two additional interactions (analyte-sorbent and solvent-sorbent) are present. The interaction between BPA and the carbon nanotubes is constant regardless of what solvent is chosen for desorption, however, the interaction between desorption solvent and CNTs is different for each solvent. The interactions between BPA and the desorption solvent must be strong enough that BPA can be removed from the carbon nanotube surface. In addition, the interaction between CNTs and desorption solvent must be sufficient enough that the solvent will replace the BPA molecules on the nanotube surface. Although the solubility of BPA in methanol and acetonitrile is similar, the interactions between BPA, CNTs and methanol are greater. Likely, methanol is more capable of wetting CNTs and therefore the solvent-sorbent interaction is greater than when acetonitrile is used. 
Furthermore, the ideal excitation and emissions wavelengths for maximum BPA emission were only determined in methanol. Since BPA may fluoresce at slightly different excitation or emission wavelengths in different solvents, the amount of BPA detected in acetonitrile may have been higher with different detector conditions. The extraction capability of acetone could not determined experimentally using HPLC with fluorescence detection. Acetone absorbs UV light below $335 \mathrm{~nm}$ and when an acetone solution containing BPA was analyzed by HPLC, a negative peak (below the baseline) was observed. Since the maximum solubility of BPA was similar in acetone and methanol, and it has been observed acetone has a strong ability to wet carbon nanotubes, the desorption efficiency of methanol and acetone were likely similar. 


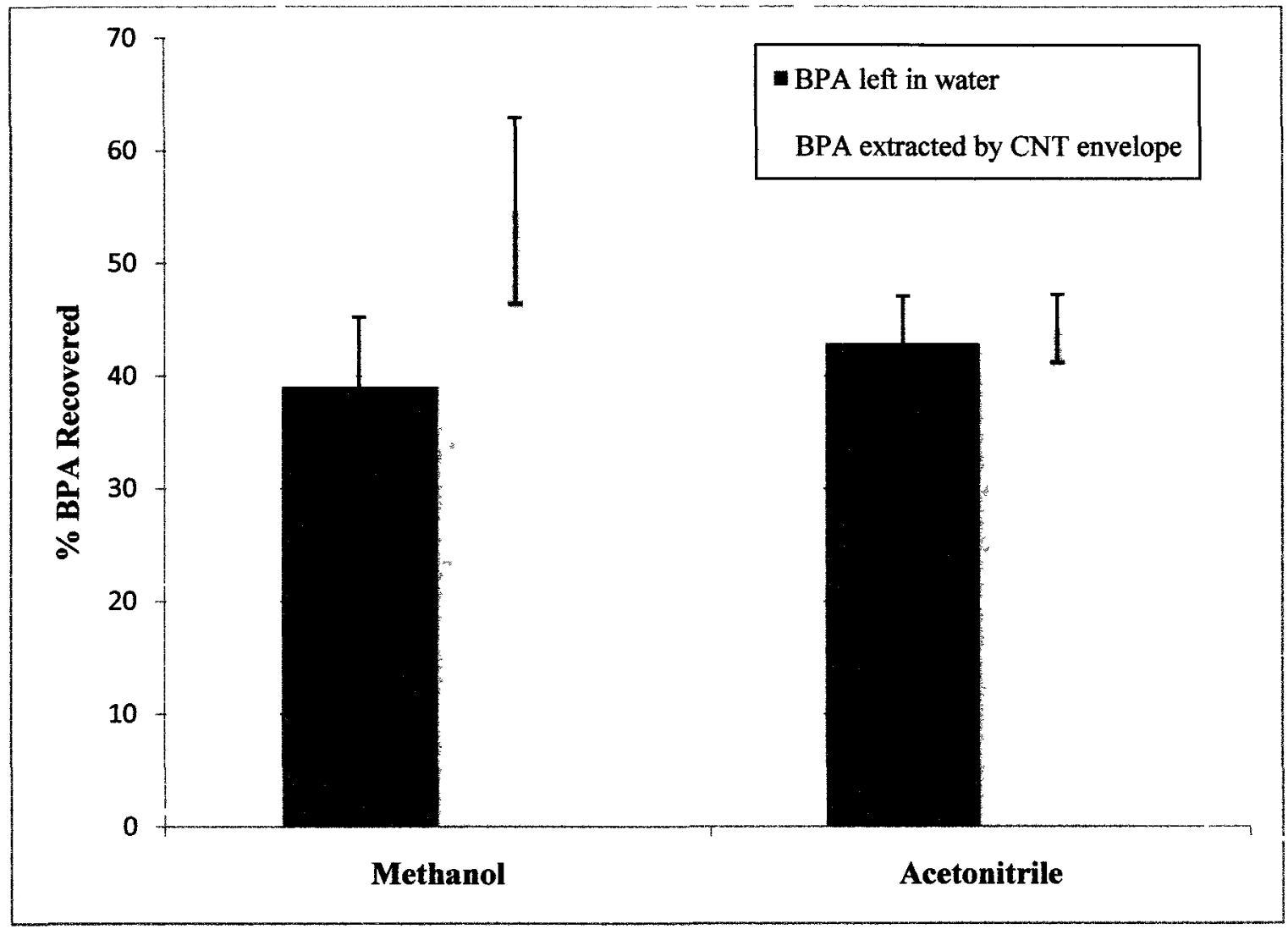

Figure 3-64 Comparison of desorption solvents for BPA elution from the PVDF-CNT envelopes.

\subsection{BPA Extraction from Baby Bottles Using PVDF-CNT Envelopes}

Since the youngest members of the population are affected most by BPA exposure and the effects of exposure on infants and young children cannot be studied experimentally [21,22], it is important to develop effective analytical methods to test for BPA exposure in infants. The Government of Canada's [63] recent ban on the production, sale and importation of polycarbonate baby bottles containing BPA indicates one of the most common and likely most abundant exposure routes to this part of the population are these bottles. Therefore, it was decided that a practical application of BPA analysis of water in 
contact with baby bottles using CNT envelopes would be an effective "real life" test of CNT envelopes.

Several baby bottles were obtained for these experiments. In view of the recent legislation against BPA-containing baby bottles in Canada, all major baby bottle suppliers now indicate that their baby bottles are \%100 BPA Free. These suppliers sell baby bottles across North America and consequently baby bottles containing BPA could not easily be found in Canada or the US. As a result, baby bottles from outside North America were sought after in hopes they may contain BPA. Six new bottles were purchased from local stores in China and Thailand. The packaging did not indicate if the bottles did or not did not contain BPA, however inexpensive bottles were chosen since it is likely more cost effective to use the BPA monomer in polycarbonate synthesis than other alternatives. In addition, used baby bottles of major North American brands were donated from another research group. These suppliers include those who now advertise their baby bottles as BPA-free. Although the origin of these bottles is not exactly known, they were likely purchased at local stores in Canada roughly five years ago, prior to the ban of BPA. A photograph of the bottles used for this practical application of BPA extraction using PVDF-CNT envelopes is shown below in Figure 3-65. 


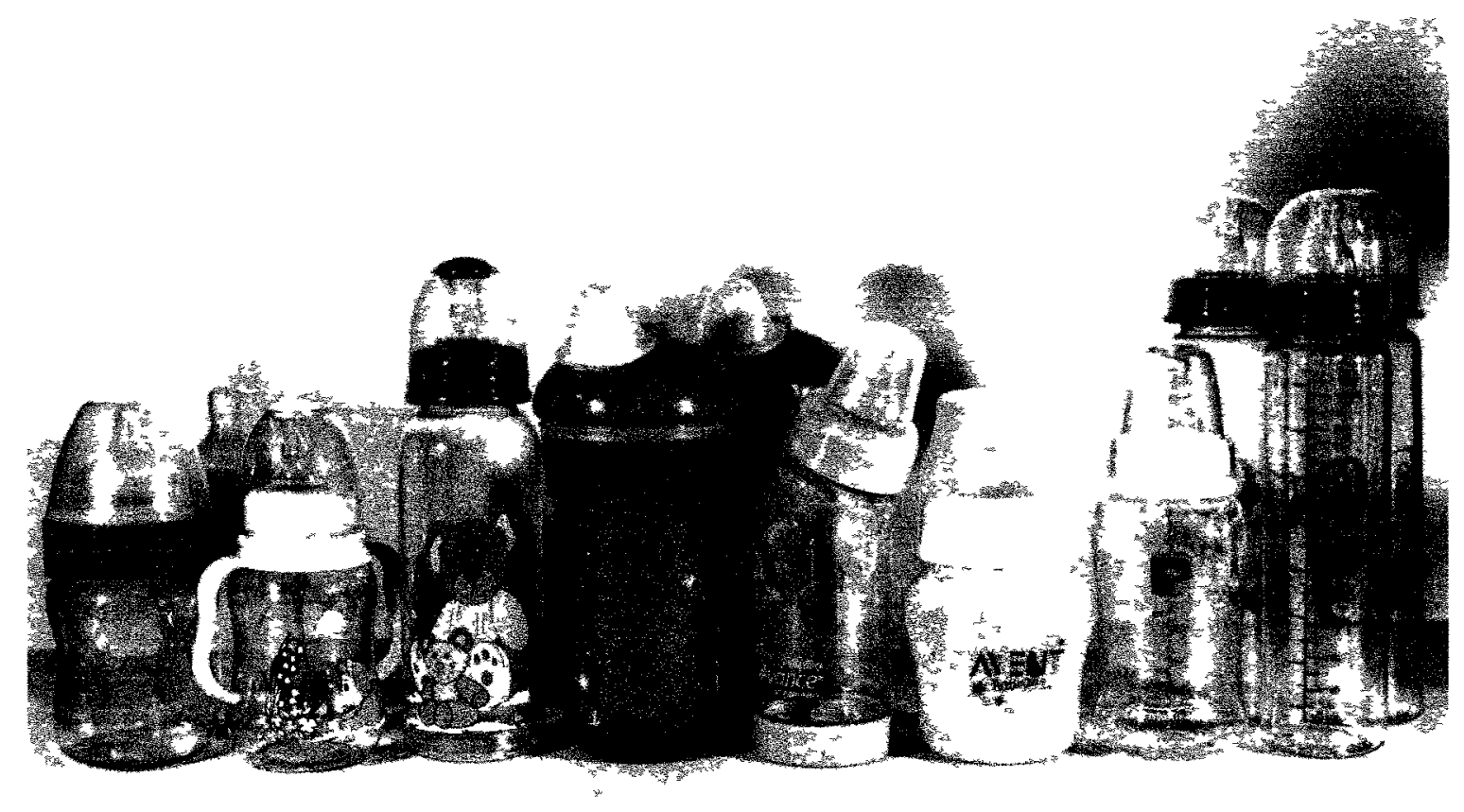

Figure 3-65 Baby bottles used for practical application of BPA extraction from aqueous solution using PVDF-CNT envelopes. From left: A1, A2 (behind A1), B1, C1, D1 (orange), D2 (purple, behind D1), E1, F1, G1, H1, H2 and H3 (both behind H1).

To begin, three extractions in deionized water were performed to serve as a blank. Since almost all experiments were carried out in a sample volume of $40 \mathrm{~mL}$, this volume was also used for extraction in deionized water. After exposure to deionized water, the PVDF-CNT envelopes were transferred to $1 \mathrm{~mL}$ of methanol to concentrate any BPA extracted. HPLC analysis of the methanol extracts indicated no BPA was extracted from deionized water.

Since it could not be confirmed if any of the bottles obtained were synthesized with BPA, several different extraction techniques were employed to potentially leach BPA out of the plastic, if the bottle did in fact contain BPA. Leaching was examined with oven and 
microwave heating and at room temperature. Generally, the same procedure was repeated on two bottles, one of the used bottles and one of the new bottles.

First, deionized water was heated to $85^{\circ} \mathrm{C}$ and then used to fill two bottles (labeled $\mathrm{A} 1$ and $\mathrm{B} 1$ in Figure 3-65) which were subsequently placed in oven at $85^{\circ} \mathrm{C}$ for 30 minutes. After cooling to room temperature, the solutions were transferred to volumetric flasks where they were stored until further use, to prevent continuous leaching. Since it was demonstrated exhaustive extraction is approached at small sample volumes using PVDFCNT envelopes, adsorption was performed in the smallest volume that could be achieved with stirring, $15 \mathrm{~mL}$. Similarly, desorption was performed in the smallest volume of methanol that did not compromise extraction efficiency, $1 \mathrm{~mL}$.

The concentration of BPA extracted by the CNT envelope for both A1 and B1 was so low it could not be integrated by the detector, although a peak was visible in the chromatogram. Instead of using a calibration curve, the concentration of BPA extracted was determined by preparing a standard addition curve. A small volume of a $250 \mathrm{ng} / \mathrm{mL}$ BPA in methanol standard was added to the BPA extract to increase the magnitude of the BPA peak so it could be detected. The concentration of the solution was analyzed by HPLC between each standard addition. A standard addition curve of peak area versus concentration of BPA added was plotted to determine the concentration of BPA in the extract, the negative value of the $x$-intercept. Figure 3-66 shows the standard addition curves for both bottles. The concentration of BPA in A1 and B1 was determined to be $73 \mathrm{ng} / \mathrm{mL}$ and $19 \mathrm{ng} / \mathrm{mL}$, respectively. 


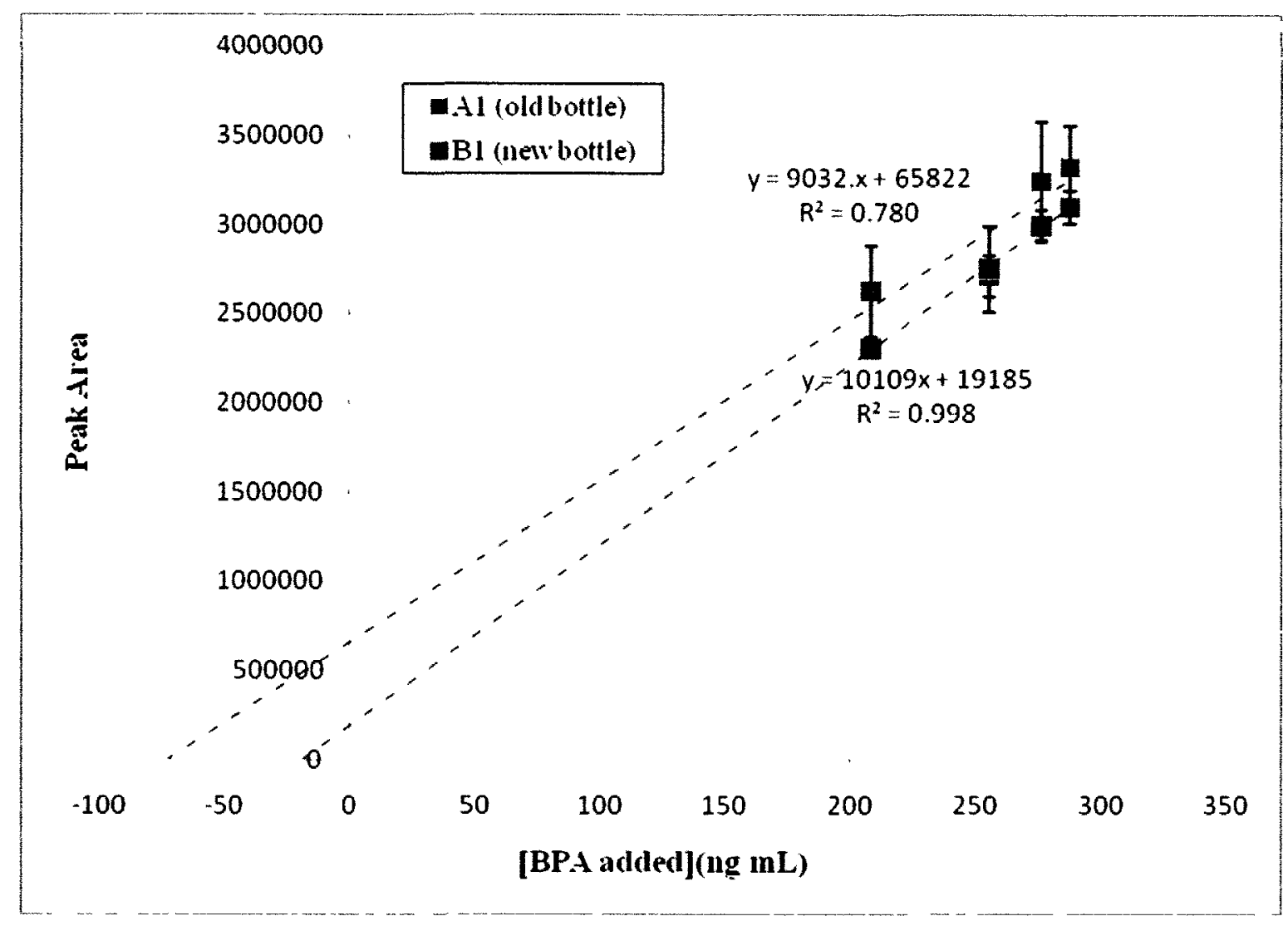

Figure 3-66 Standard addition curves of old bottle A1 and new bottle B1 to determine the amount of BPA leached from the bottles into aqueous solution.

Repeated extractions using the same bottles were performed to determine the amount of BPA that leached out of the plastic bottle after successive uses. Each time, the water from the previous extraction was transferred back to the bottle to determine the total amount of BPA leaching from the bottle. In other words, the water in the bottle during leaching was never discarded so that the leaching and extraction processes were repeated in the same aqueous solution as the previous extraction. However, since BPA is significantly more soluble in basic solution, a $\mathrm{NaOH}$ pellet was added to the flask to increase the $\mathrm{pH}$ of the solution during leaching, for the second time. Later, during 
adsorption, a few drops of concentrated $\mathrm{HCl}$ were added to the water to make the aqueous solution acidic and thus promote BPA adsorption to the envelope.

As the amount of BPA leached out of the bottle increased, the HPLC increased signal to range that could be integrated. As the concentration of BPA increased, a second peak was also evident in the chromatogram, which overlapped with the BPA peak, as shown in Figure 3-67. This second peak was present in every extract and as a result, the BPA peak could not be integrated. To separate the two peaks, the composition of the mobile phase was changed to $50 \% \mathrm{MeOH}: 50 \% \mathrm{dH}_{2} \mathrm{O}$ increasing to $100 \% \mathrm{MeOH}$ over ten minutes. Changing the composition of the gradient decreased analyte sensitivity slightly, but BPA in methanol standards were analyzed in addition to the BPA extracts from the baby bottles and thus the concentration of BPA in the extract could still be determined accurately. Figure 3-68 shows the separation between BPA (retention time $\sim 6$ minutes) and any other peaks present in the sample.

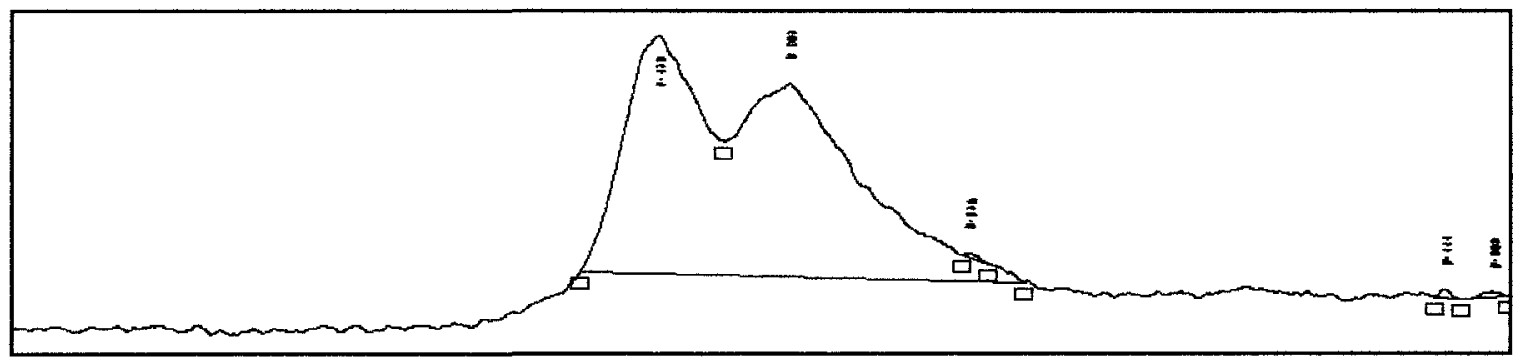

Figure 3-67 Chromatogram showing the presence of another analyte overlapping with BPA when the BPA extract from a baby bottle was anayzed by HPLC. The composition of the mobile phase was $90 \% \mathrm{MeOH}: 10 \% \mathrm{dH}_{2} \mathrm{O}$ to start. 


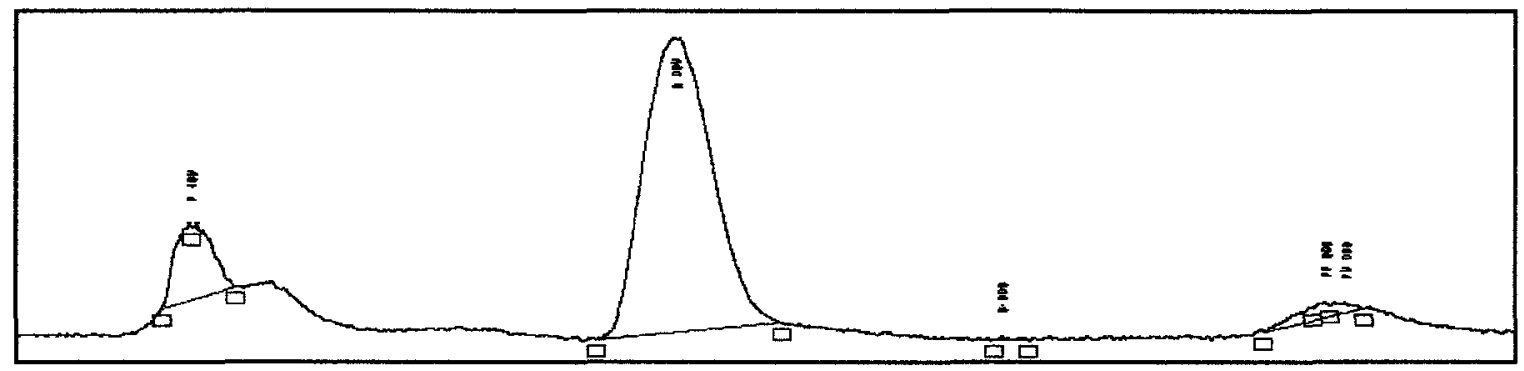

Figure 3-68 Chromatogram showing the separation between BPA and any other analytes when the composition of the mobile phase was changed to $50 \% \mathrm{MeOH}: 50 \% \mathrm{dH} 2 \mathrm{O}$ to start.

Since it was confirmed BPA leached in water bottle A1, extraction with a second old bottle of the same make, A2, was performed and compared to the first. The results of the BPA extraction from A1 and A2 are shown in Figure 3-69. As seen, the amount of BPA increased with each successive extraction, since the bottles were filled with the same water repeatedly during leaching. Since both of these bottles had been used previously, it is not known if they were used the same amount or if the milk served in them was prepared (heated or not) the same way, thus discrepancies between leaching concentrations it to be expected. This plot indicates BPA leaches from the bottles over successive uses and that when the aqueous solution is basic (all extractions except the first) the amount of BPA leached into water is greater. 


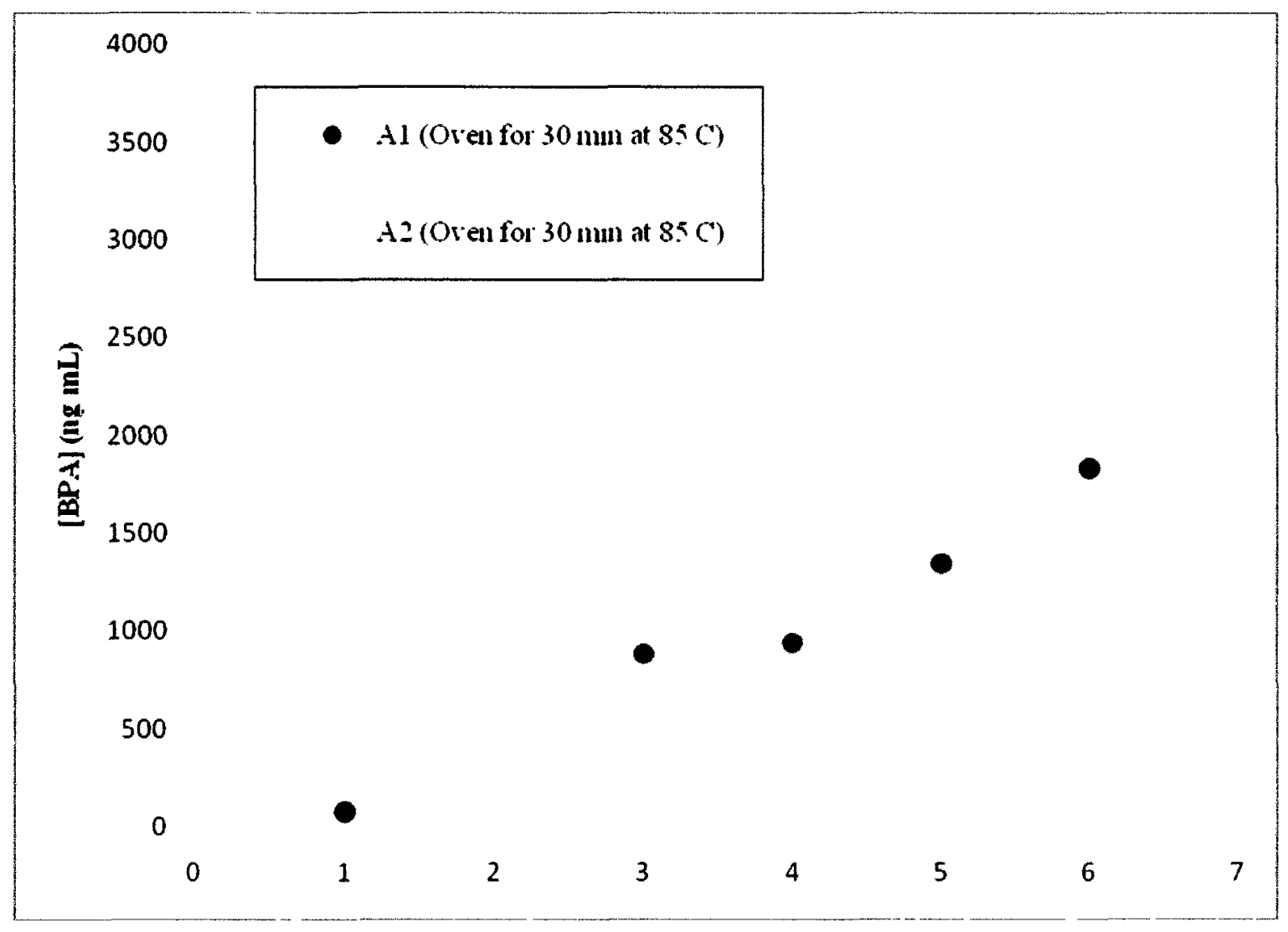

Figure 3-69 Concentration of BPA leached from old bottles A1 and A2 after several successive extractions.

The amount of BPA released from the new plastic, B1, during heating from the oven, was significantly greater than the two used bottles, A1 and A2. After five successive extractions the concentration of BPA in the aqueous solution in the new bottle was $33 \pm$ $16 \mu \mathrm{g} / \mathrm{mL}$. Both $\mathrm{B} 1$ and $\mathrm{C} 1$ were purchased in China and although they are the same brand, extractions using these bottles could not be compared directly since they are different sizes. Since the concentration of aqueous solution in B1 was so much higher than the results obtained from the old bottles, $\mathrm{C} 1$ also likely contained a significant amount of BPA. BPA leaching from this bottle was only measured once to determine the amount of BPA that could be extracted under harsh, prolonged conditions. The bottle 
was filled with basic water $(\sim \mathrm{pH} 12)$ and maintained at a temperature of $85^{\circ} \mathrm{C}$ for 24 hours. The concentration of BPA removed after only a single extraction at these conditions was $98 \pm 7 \mu \mathrm{g} / \mathrm{mL}$.

The amount of BPA leached from polycarbonate baby bottles into aqueous solution was also examined at room temperature. Two old bottles, D1 and D2, and one new bottle, E1, were filled with basic water and left on the lab bench for 24 hours. Extraction was performed as mentioned above. The amount of BPA extracted after the first extraction for both bottles was below the detection limit of the instrument and could not be quantified, thus the results of BPA leaching from D1 and D2 at room temperature do not include the first extraction, as shown in Figure 3-70. Once again, both of these bottles were used previously and the extent of use is not known. Therefore, discrepancies in the amount of BPA leached between bottles are to be expected. The amount of BPA that leached from the new bottle, $\mathrm{E} 1$, at room temperature was determined to be $4 \pm 2 \mu \mathrm{g} / \mathrm{mL}$ (not shown in Figure 3-70). 


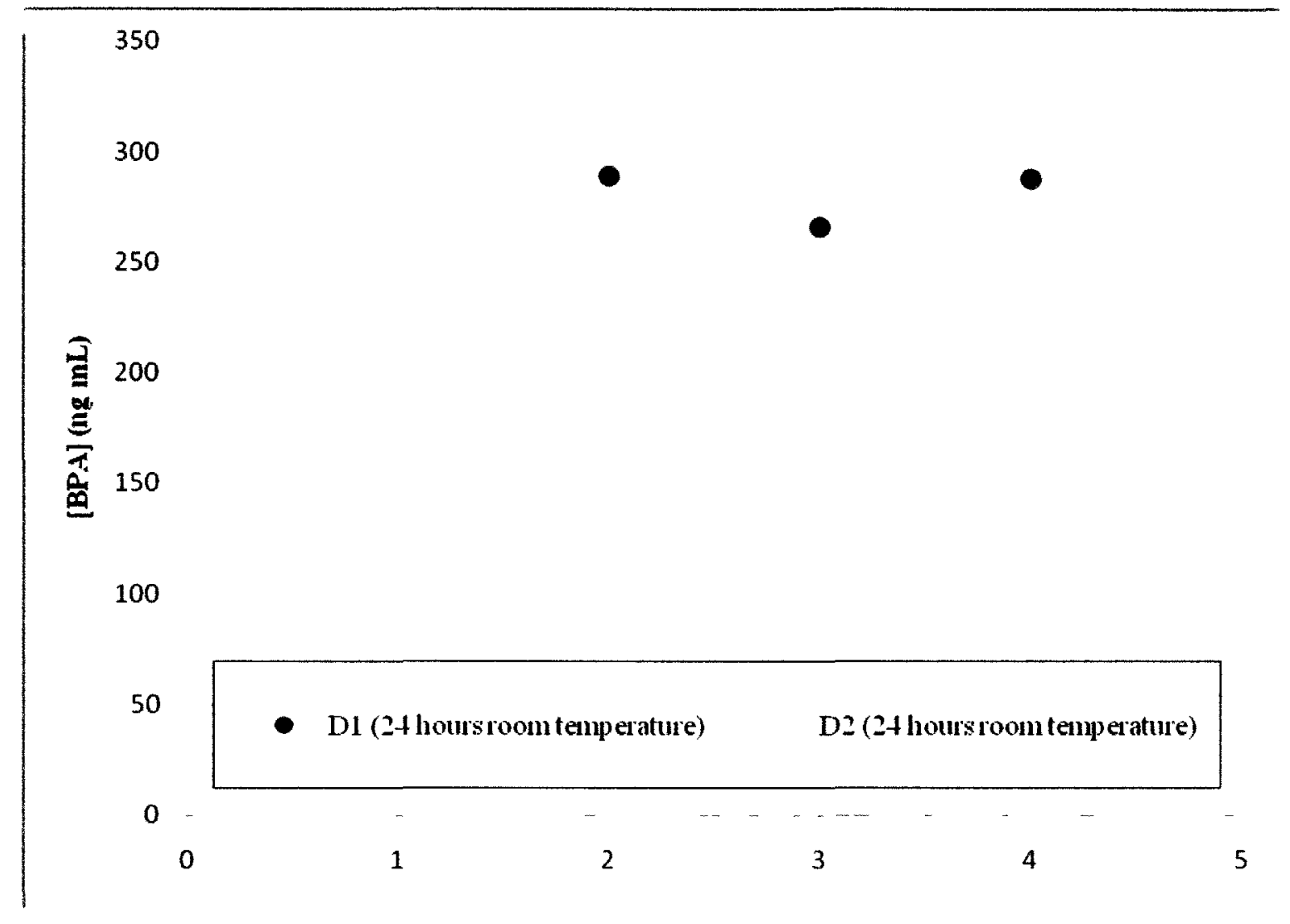

Figure 3-70 Concentration of BPA leached into water from old bottles D1 and D2 after several successive extractions.

BPA extraction was also examined when polycarbonate baby bottles filled with basic aqueous solution were heated in a microwave. Two bottles, F1 and G1 were filled with basic deionized water and were heated in a microwave for 45 seconds. Adsorption and desorption were carried out in the same volumes as described above. The average concentration of BPA in the aqueous solution from F1 after five extractions was determined to be $66 \pm 48 \mathrm{ng} / \mathrm{mL}$, while the average concentration in $\mathrm{G} 1$ after four extractions was determined to be $820 \pm 300 \mathrm{ng} / \mathrm{mL}$.

The error associated with the concentration of BPA in each solution is significant. Since the water used to fill the bottle is reused from one extraction to the next, the amount of 
BPA leaching into aqueous solution may be affected since the solution already contains BPA. To determine the reproducibility of BPA extraction from polycarbonate bottles using PVDF-CNT envelopes, basic aqueous solution was heated to $50^{\circ} \mathrm{C}$ and transferred to three new bottles, $\mathrm{H} 1-\mathrm{H} 3$, for leaching. The bottles were left on the lab bench to cool, instead of being transferred to the oven, to more closely mimic the real-life conditions these bottles would be used for. Adsorption and desorption were performed as previously discussed. The average amount of BPA extracted from these three bottles was determined to be $720 \pm 80 \mathrm{ng} / \mathrm{mL}$. Although the error associated with this value is still large, it is more reasonable than the previously reported measurements.

The average mass of BPA leached from each bottle is shown below in Table 3-4. As shown, the amount of BPA leached from the new, unused bottles is an order of magnitude greater than the mass of BPA extracted from the used bottles. 
Table 3-4 Mass of BPA extracted from each baby bottle.

\begin{tabular}{|c|c|c|}
\hline Bottle Label & $\begin{array}{c}\text { BPA leaching conditions over } n \\
\text { extractions }\end{array}$ & $\begin{array}{l}\text { Mass BPA extracted at } \\
\text { equilibrium* }(\mu \mathrm{g})\end{array}$ \\
\hline $\begin{array}{c}\text { A1 } \\
\text { (old) }\end{array}$ & $\begin{array}{c}30 \text { min oven at } \\
n=6\end{array}$ & 210 \\
\hline $\begin{array}{l}\text { A2 } \\
\text { (old) }\end{array}$ & $\underset{n=5}{30 \text { min oven at } 85^{\circ} \mathrm{C}}$ & 250 \\
\hline $\begin{array}{c}\text { B1 } \\
\text { (new) }\end{array}$ & $\begin{array}{c}30 \text { min oven at } 85^{\circ} \mathrm{C} \\
n=5\end{array}$ & 5300 \\
\hline $\begin{array}{c}\mathrm{C} 1 \\
\text { (new) }\end{array}$ & $\begin{array}{c}24 \text { hours oven at } 85^{\circ} \mathrm{C} \\
n=1\end{array}$ & 26500 \\
\hline $\begin{array}{l}\text { D1 } \\
\text { (old) }\end{array}$ & $\begin{array}{l}24 \text { hours room temperature } \\
n=4\end{array}$ & 45 \\
\hline $\begin{array}{l}\mathrm{D} 2 \\
\text { (old) }\end{array}$ & $\begin{array}{l}24 \text { hours room temperature } \\
\qquad n=4\end{array}$ & 60 \\
\hline $\begin{array}{c}\text { E1 } \\
\text { (new) }\end{array}$ & $\begin{array}{l}24 \text { hours room temperature } \\
\qquad \mathrm{n}=3\end{array}$ & 1000 \\
\hline $\begin{array}{c}\text { F1 } \\
\text { (old) }\end{array}$ & $\begin{array}{c}45 \mathrm{sec} \text { microwave } \\
\mathrm{n}=5\end{array}$ & 25 \\
\hline $\begin{array}{c}\text { G1 } \\
\text { (new) }\end{array}$ & $\begin{array}{c}45 \mathrm{sec} \text { microwave } \\
\mathrm{n}=4\end{array}$ & 180 \\
\hline $\begin{array}{c}\mathrm{H} 1 \\
\text { (new) }\end{array}$ & $\begin{array}{l}\text { Filled with } \mathrm{H}_{2} \mathrm{O} \text { at } 50^{\circ} \mathrm{C} \text {, left to cool } \\
\qquad \mathrm{n}=1\end{array}$ & 145 \\
\hline $\begin{array}{c}\mathrm{H} 2 \\
\text { (new) }\end{array}$ & $\begin{array}{l}\text { Filled with } \mathrm{H}_{2} \mathrm{O} \text { at } 50^{\circ} \mathrm{C} \text {, left to cool } \\
\qquad \mathrm{n}=1\end{array}$ & 170 \\
\hline $\begin{array}{c}\mathrm{H} 3 \\
\text { (new) }\end{array}$ & $\begin{array}{l}\text { Filled with } \mathrm{H}_{2} \mathrm{O} \text { at } 50^{\circ} \mathrm{C} \text {, left to cool } \\
\qquad \mathrm{n}=1\end{array}$ & 180 \\
\hline
\end{tabular}

*The amount of BPA extracted did not reach equilibrium for all bottles, and as such the mass of BPA extracted was determined from the concentration of BPA in the last extraction. 
The no observed adverse effect level (NOAEL) for BPA has been set at $5 \mathrm{mg} / \mathrm{kg} \bullet \mathrm{bw} / \mathrm{day}$ in North America and Europe [36,37]. The mass of BPA leached from all bottles was lower than $5 \mathrm{mg}$ however when compared against the provisional tolerable daily intake as outlined by Health Canada ( $25 \mu \mathrm{g} / \mathrm{kg} \cdot \mathrm{bw} / \mathrm{day})$ [62], the mass of BPA leached from each polycarbonate baby bottle is significant. For example, when bottle $\mathrm{C} 1$ is considered, the amount of BPA leached from the bottle is more than 1000 times the tolerable daily intake for BPA. The leaching conditions for bottles H1-H3 most closely mimic a "real-life" situation for serving newborns and infants with a polycarbonate baby bottle. The average mass of BPA recovered from these three bottles, $165 \mu \mathrm{g}$, is almost 7 times the tolerable daily intake. If the mass of a newborn baby is considered, $(<5 \mathrm{~kg})$ the mass of BPA recovered from one bottle, which also only represents one serving, has already exceeded the tolerable daily intake. As mentioned, despite a NOAEL in the $\mathrm{mg} / \mathrm{kg} \cdot \mathrm{bw} / \mathrm{day}$ range, laboratory studies on rodents have indicated observable adverse effects in adult rodents in the $\mu \mathrm{g} / \mathrm{kg} \cdot \mathrm{bw} /$ day range and negative responses as low as $\mathrm{ng} / \mathrm{kg} \bullet \mathrm{bw} / \mathrm{day}$ when fetuses were exposed in utero. As further evidence is put forth clarifying the low-dose effect controversy surrounding BPA exposure, sensitive and reliable analytical tools will be needed for BPA detection in the environment. The studies shown here indicate PVDFCNT envelopes are a viable choice for solid-phase extraction of BPA from aqueous solution. 


\section{CONCLUSION}

Solid-phase extraction of BPA from aqueous solutions was successful using CNT envelopes. CNT envelopes were easily fabricated with a set of stainless steel tools designed by the Science Technology Center at Carleton University.

The dimensions of the CNTs used were characterized by scanning and transmission electron microscopy. Surface area measurements indicated an increase in surface area with smaller nanotube diameter. Infrared spectroscopy was used to characterize oxidized single and multi-walled CNTS, however no oxygen-containing stretches characteristic of CNT oxidation were observed. Aqueous suspensions of pristine and oxidized SWCNTs and MWCNTs were prepared to confirm nanotube oxidation.

BPA extraction directly from aqueous solution was not successful using CNT envelopes prepared from the hydrophobic polypropylene membrane. The use of envelope conditioning prior to extraction was explored and recovery increased significantly when the device was conditioned with polar solvents, particularly methanol and acetone. A comparison of BPA extraction with empty, conditioned PP envelopes revealed the empty envelope was also capable of significant BPA extraction $(>50 \%$ in acetone and methanol). Exploration of a few types of conditioning techniques indicated solvent-BPA interactions existed, regardless of conditioning technique, so BPA adsorption to conditioned PP-CNT envelopes could not be isolated to just the CNTs. As a result, use of PP-CNT envelopes for BPA extraction was abandoned. 
BPA extraction using CNT envelopes made with the hydrophilic polyvinylidene fluoride membrane was successful. Initial BPA recovery was found to be $\sim 55 \%$. Study of adsorption over time indicated equilibrium was not reached until about 9.25 hours. The lengthy adsorption time indicated diffusion through the membrane was likely the rate limiting factor. Study of adsorption over time without stirring demonstrated the use of agitation improves the adsorption rate. BPA adsorption was found to be a pseudo-first order process. Extraction closely resembled SPME as it was determined to be an equilibrium extraction, rather than an exhaustive process.

Evaluation of extraction efficiency at different temperatures indicated BPA adsorption increases at higher temperatures. The enthalpy and entropy of the overall process of moving BPA from water to the nanotube surface system was determined from a van Hoff plot. $\Delta \mathrm{H}^{\circ}(37 \mathrm{~kJ} / \mathrm{mol})$ indicated $\mathrm{BPA}$ the overall reaction is endothermic and $\Delta \mathrm{S}^{\circ}(200 \mathrm{~J} /$ $\mathrm{K} \cdot \mathrm{mol})$ revealed the moving BPA from water to CNTs is less ordered. Further information is needed to deduce conclusions about the enthalpy and entropy of forming the BPA-CNT bond.

BPA adsorption was explored over a wide range of sample concentration and the limit of detection of the envelope was determined to be $0.5 \mathrm{ng} / \mathrm{mL}$. At low concentrations, adsorption was more appropriately described as an exhaustive extraction process (rather than equilibrium extraction) as the CNT envelope removed almost all BPA present in the aqueous sample solution. BPA recovery was also examined over a wide sample volume range and in small sample sizes; adsorption was also determined to be exhaustive. 
Varying the $\mathrm{pH}$ of the sample solution did not affect extraction efficiency in the range $\mathrm{pH}$ 2-8, however, extraction decreased above $\mathrm{pH} 8$ due to deprotonation of the $-\mathrm{OH}$ groups.

A study of CNT mass inside the envelope indicated the mass of CNTs used $(\sim 6.45 \mathrm{mg})$ was more than sufficient to extract BPA from aqueous solution. Comparison of envelopes filled with single, double or multi-walled CNTs indicated BPA adsorption to SWCNTs was greatest, likely due to the increased curvature of the tubes due to its small diameter, compared to nanotubes with greater diameter. Comparison of pristine and oxidized CNTs indicated BPA adsorption to pristine CNTs is more favourable.

The rate of desorption was examined with and without stirring, and equilibrium was observed within 10 minutes, regardless of whether stirring was used. BPA could be concentrated in as little as $1 \mathrm{~mL}$ of methanol without stirring. When desorption was explored with methanol and acetonitrile, methanol was found to be a better desorption solvent.

PVDF-CNT envelopes were successfully used to determine the mass of BPA leached into aqueous solution from polycarbonate baby bottles. The mass of BPA recovered was dependent on the state of the bottle, but generally, the less a bottle had been used (new versus old) the more BPA was extracted from it. 


\section{REFERENCES}

1. Iijima, S. Helical microtubes of graphitic carbon. Nature. 1991, 354, 56-58.

2. Iijima, S.; Ichihashi, T. Single-shell carbon nanotubes of 1-nm diameter. Nature. $1993,363,603-605$.

3. Valcárcel, M.; Simonet, B. M.; Cárdenas, S.; Suárez, B. Present and future applications of carbon nanotubes to analytical science. Anal. Bional. Chem. $2005,382,1783-1790$.

4. Merkoçi, A. Carbon nanotubes in analytical sciences. Microchim. Acta. 2006, $152,157-174$.

5. Dai, H. Carbon nanotubes: opportunities and challenges. Surface Science. 2002, $500,218-241$.

6. Ebbesen, T. W. Carbon Nanotubes. Amu. Rev. Mater. Sci. 1994, 24, 235-264.

7. Belin, T.; Epron, T. Characterization methods of carbon nanotubes: a review. Materials Science and Engineering B. 2005, 119, 105-118.

8. Ebbesen, T. W; Ajayan, P. M. Large scale synthesis of carbon nanotubes. Nature. 1994, 358, 220-222.

9. Colbert, D. T.; Zhang, J.; McClure; S. M. Nikolaev; P.; Chen, Z.;Hafner, J. H.; Owens, D. W.; Kotula, P. G.; Carter, C. B.; Weaver, J. H.; Rinzler, A. D.; 
Smalley, R. E. Growth and sintering of fullerene nanotubes. Science. 1994, 266, 1218-1222.

10. Ando, Y. Preparation of carbon nanotubes. Fullerene Sci. Tech. 1994, 2, 173 180.

11. Zhao, X.; Ohkohchi, M.; Wang, M.; Iijima, S.; Ichihashi, T.; Ando, Y. Preparation of high-grade carbon nanotubes by hydrogen arc discharge. Carbon. $1997,34,775-781$.

12. Sen, R.; Ohtsuka, Y.; Ishigaki, T.; Kasuya, D.; Suzuki, S.; Kataura, H.; Achiba, Y. Time period for the growth of single-wall carbon nanotubes in the laser ablation process: evidence from gas dynamic studies and time resolved imaging. Chem. Phys. Lett. 2000, 243, 49.

13. Couteau, E.; Hernadi, K.; Seo, J. W.; Thien-Nga, L.; Miko, C.; Gaal, R.; Forro, L. CVD synthesis of high-purity multiwalled carbon nanotubes using $\mathrm{CaCO}_{3}$ catalyst support for large-scale production. Chem. Phys. Lett. 2003, 378, 9-17.

14. Balasubramanian. K.; Burghard, M. Chemically Functionalized Carbon Nanotubes. Small. 2005, 1, 2, 180-192.

15. Kuzmany, H.; Kukovecz, A.; Simon, F.; Holzweber, M.; Kramberger, C.; Pichler, T. Functionalization of carbon nanotubes. Synthetic Metals. 2004, 141, 113122. 
16. Hu, H.; Bhowmik, P.; Zhao, B.; Hamon, M. A.; Itkis, M. E.; Haddon, R. C. Determination of the acidic sites of purified single-walled carbon nanotubes by acid-base titration. Chem. Phys. Lett. 2001, 345, 25-28.

17. Chen, J.; Hamon, M. A.; Hu, H.; Chen, Y.; Rao, A. M.; Eklund, P. C.; Haddon, R. C.; Solution Properties of Single-Walled Carbon Nanotubes. Science. 1998, $292,95-98$.

18. Banerjee, S.; Hemraj-Benny, T.; Wong S. S. Covalent Surface Chemistry of Single-Walled Carbon Nanotubes. Adv. Mater. 2005, 17 (1), 17-29.

19. Cousins, I. T.; Staples, C. A.; Klečka, G. M.; MacKay, D. A multimedia assessment of the environmental fate of bisphenol A. Human and Ecological Risk Assessment. 2002, 8 (5), 1107-1135.

20. Staples, C. A.; Dosn, P. B.; O'Block, S. T.; Harris, L. R. A review of the environmental fate, effects and exposures of bisphenol A. Chemosphere. 1998, $36(10), 2149-2173$.

21. vom Saal FS, Akingbemi BT, Belcher SM, Birnbaum LS, Crain DA, Eriksen M, Farabollini F, Guillette LJ Jr, Hauser R, Heindel JJ, Ho SM, Hunt PA, Iguchi T, Jobling S, Kanno J, Keri RA, Knudsen KE, Laufer H, LeBlanc GA, Marcus M, McLachlan JA, Myers JP, Nadal A, Newbold RR, Olea N, Prins GS, Richter CA, Rubin BS, Sonnenschein C, Soto AM, Talsness CE, Vandenbergh JG, Vandenberg LN, Walser-Kuntz DR, Watson CS, Welshons WV, Wetherill Y, Zoeller RT. Chapel Hill bisphenol A expert panel consensus statement: 
integration of mechanisms, effects in animals and potential to impact human health at current levels of exposure. Reprod. Toxicol. 2007, 24 (2), 131-138.

22. Vandenberg, L.N.; Maffini, M. V.; Sonnenschein, C.; Rubin, B. S.; Soto, A. M. Bisphenol A and the great divide: a review of controversies in the field of endocrine disruption. Endocrine Reviews. 2009, 30 (1), 75-95.

23. Burridge, E. Bisphenol A: product profile. Eur. Chem. News. 2003, 17.

24. Spivack, J.; Leib, T. K.; Lobos, J. H. Novel pathway for bacterial metabolism of bisphenol A, rearrangements and stilbene cleavage in bisphenol A metabolism. J. Biol. Chem. 1994, 269, 7323-7329.

25. Ike, M.; Chen, M. Y.; Jin, C. S.; Fujita, M. Acute toxicity, mutagenicity, and estrogenicity of biodegradation products of bisphenol-A. Environ. Toxicol. $2002,17(5), 457-461$.

26. Klecka, G. M.; Gonsior, S. J.; West, R. J.; Goodwin, P. A.; Markham, D. A. Biodegradation of bisphenol A in aquatic environments: river die-away. Environ Toxicol Chem. 2001, 12, 2725-35.

27. Gouin, T.; Mackay, D.; Webster, E.; Wania, F. Screening chemicals for persistence in the environment. Environ. Sci. Technol. 2000, 34, 881-884.

28. Calafat, A. M.; Ye, X.; Wong, L. Y.; Reidy, J. A.; Needham, L.L. Exposure of the U.S. population to bisphenol A and 4-tertiary-octylphenol. Environmental Health Perspectives. 2008, 116, 39-44. 
29. Becker, K.; Göen, T.; Seiwart, M. German Environmental Survey for Children 2003/06 (GerES IV): Phthalate metabolites and bisphenol A in urine of German children. International Journal of Hygiene and Environmental Health. 2009, 212, 685-692.

30. Beronius, A.; Rudén, Håkansson, H.; Hanberg, A. Risk to all or none? A comparative analysis of controversies in the health risk assessment of bisphenol A. Reproductive Toxicology. 2010, 29, 132-146.

31. Kang, J. H.; Kondo, F.; Katayama. Human exposure to bisphenol A. Toxicology. 2006, 226, 79-89.

32. Opinion of the scientific committee on food on bisphenol A. 2002. http://europa.eu.int/comm/food/fs/sc/scf/out128_en.pdf

33. IUPAC Compendium of Chemical Terminology. 1993, 65, 2076. http://old.iupac.org/goldbook/N04208.pdf

34. Tyl, R. W.; Myers, C. B.; Marr, M. C.; Thomas, B. F.; Keimowitz, A. R.; Brine, D. R.; Veselica, M. M.; Fail, P. A.; Chang, T. Y.; Seely, J. C.; Joiner, R. L.; Butula, J. H.; Dimond, S. S.; Cagen, S. Z.; Shiotsuka, R. N.; Stropp, G. D.; Waechter, J. M. Three-generation reproductive toxicity study of dietary bisphenol A in CD Sprague-Dawley rats. Toxicol. Sci. 2002, 68, 121-146.

35. R.W. Tyl, C.B. Myers, M.C. Marr, C.S. Sloan, N.P. Castillo and M.M. Veselica, Tyl, R. W.; Myers, C. B.; Marr, M. C.; Sloan, C. S.; Veselica, M. M.; Seely, J. C.; Dimond, S. S.; van Miller, J. P.; Shiotsuka, R. N.; Beyer, D.; Hentges, S. G.; 
Waechter, J. M. Two-generation reproductive toxicity study of dietary bisphenol A in CD-1 (Swiss) mice. Toxicol. Sci. 2008, 104, 362-384.

36. European Food Safety Authority. Opinion of the Scientific Panel on Food Additives, Flavourings, Processing Aids and Materials in Contact with Food on a request from the Commission related to 2,2-bis(4-hydroxyphenyl)propane (bisphenol A). 2006. www.efsa.europa.eu

37. US Food and Drug Administration. Draft assessment of bisphenol A for use in food contact applications. 2008. www.fda.gov.

38. Kuiper, G.G.; Lemmen, J. G.; Carlsson, B.; Corton, J.C.; Safe, S. H.; Van Der Saag, P. T.; van der Burg, B.; Gustafsson, J. A. Interaction of estrogenic chemicals and phytoestrogens with estrogen receptor $\beta$. Endocrinology. 1998, $139,4252-4263$.

39. Andersen, H. R.; Andersson, A. M.; Arnold, S. F.; Autrup, H.; Barfoed, M.; Beresford, N. A.; Bjerregaard, P.; Christiansen, L. B.; Gissel, B.; Hummel, R.; Jorgensen, E. B.; Korsgaard, B.; Le Guevel, R.; Leffers, H.; McLachlan, J,; Moller, A,; Nielsen, J. B,; Olea, N,; Oles-Karasko, A,; Pakdel, F,; Pedersen, K. L,; Perez, P,; Skakkebaek, N. E,; Sonnenschein, C,; Soto, A. M,; Sumpter, J. P,; Thorpe, S. M,; Grandjean, P. Comparison of short-term estrogenicity tests for identification of hormone-disrupting chemicals. Environ Health Perspect. 1999, $107,89-108$. 
40. Fang, H,; Tong, W.; Perkins, R.; Soto, A. M.; Prechtl, N. V.; Sheehan, D. M. Quantitative comparisons of in vitro assays for estrogenic activities. Environ. Health Perspect. 2000, 108, 723-729.

41. Shimizu, M.; Ohta, K.; Matsumoto, Y.; Fukuoka, M.; Ohno, Y.; Ozawa, S. Sulfation of bisphenol A abolished its estrogenicity based on proliferation and gene expression in human breast cancer MCF-7 cells. Toxicol. in Vitro. 2002, $16,549-556$.

42. Richter, C.; Birnbaum, L. S.; Farabollini, F.; Newbold, R. R.; Rubin. B. S.; Talsness, C. E.; Vandenbergh, J. G.; Walser-Kuntz, D. R.; vom Saal, F. S. In vivo effects of bisphenol A in laboratory rodent studies. Reprod.Toxicol. 2007, 24, 199-224.

43. Sheehan, D. M.; vom Saal, F. S. Low dose effects of hormones: a challenge for risk assessment. . 1997, 4, 31-39.

44. Welshons, W. V.; Thayer, K. A.; Judy, B. M.; Taylor, J. A.; Curran, E. M.; vom Saal, F. S. Large effects from small exposures: I. Mechanisms for endocrinedisrupting chemicals with estrogenic activity. Environ. Health Perspect. 2003, $111,994-1006$.

45. Vandenberg, L. N.; Hauser, R.; Marcus, M.; Olea, N.; Welshons, W. V. Human exposure to bisphenol A (BPA). Reprod. Toxicol. 2007, 24, 139-177.

46. vom Saal, F. S.; Moyer, C. L. Prenatal effects on reproductive capacity during aging in female mice. Biol. Reprod. 1985, 32, 1116-1126. 
47. Ramakrishnan, S.; Wayne, N. L. Impact of bisphenol-A on early embryonic development and reproductive maturation. Reprod. Toxicol. 2008, 25, 177-183.

48. Markey, C. M.; Wadia, P. R.; Rubin, B. S.; Sonnenschein, C.; Soto, A. M. Longterm effects of fetal exposure to low doses of the xenoestrogen bisphenol-A in the female mouse genital tract. Biol. Reprod. 2005, 72, 1344-1351.

49. Taylor, J. A.; Welshons, W. V.; vom Saal, F. S. No effect of route of exposure (oral; subcutaneous injection) on plasma bisphenol A throughout 24h after administration in neonatal female mice. Reprod. Toxicol. 2008, 25, 169-176.

50. Sakamoto, H.; Yokota, H.; Kibe, R.; Sayama, Y.; Yuasa, A. Excretion of bisphenol A-glucuronide into the small intestine and deconjugation in the cecum of the rat. Biochemica. et Biophysica. Acta. 2002, 1573, 171-176.

51. Mykkanen, H; Tikka, J.; Pitkanen, T.; Hanninen, O. Fecal bacterial enzyme activities in infants increase with age and adoption of adult-type diet. J. Pediatr. Gastroenterol. Nutr. 1997, 25, 312-316.

52. Welshons, W. V.; Nagel, S.C.; vom Saal, F. S. Large effects from small exposures. III. Endocrine mechanisms mediating effects of bisphenol A at levels of human exposure. Endocrinology. 2006, 147, S56-S69.

53. Calafat, A. M.; Kuklenyik, Z.; Reidy, J. A.; Caudill, S. P.; Ekong, J.; Needham, J. L.; Urinary concentrations of bisphenol A and 4-nonylphenol in a human reference population. Environ. Health Perspect. 2005, 113, 391-395. 
54. Calafat, A. M.; Ye, X.; Wong, L. Y.; Reidy, J. A.; Needham, J. L.; Exposure of the U.S. population to bisphenol A and 4-tertiary-octylphenol: 2003-2004. Environ. Health Perspect. 2008, 116, 39-44.

55. Willhite. C. C.; Ball, G. L.; McLellan, C. J. Derivation of a Bisphenol A organ reference dose (RfD) and drinking-water equivalent concentration. J. Toxicol Environ.. Health B Crit. Rev. 2008, 11, 69-146.

56. Kawai, K.; Nozaki, T.; Nishikata, H.; Aou, S.; Takii, M.; Kubo, C. Aggressive behavior and serum testosterone concentration during the maturation process of male mice: the effects of fetal exposure to bisphenol A. Environ. Health Perspect. $2003,111,175-178$.

57. Kabuto, H.; Amakawa, M.; Shishibori, T. Exposure to bisphenol A during embryonic/fetal life and infancy increases oxidative injury and causes underdevelopment of the brain and testis in mice. Life Sci. 2004, 74, 2931-2940.

58. Welshons, W. V.; Nagel, S. C.; Thayer, K. A.; Judy, B. M.; vom Saal, F. S. Lowdose bioactivity of xenoestrogens in animals: fetal exposure to low doses of methoxychlor and other xenoestrogens increases adult prostate size in mice. Toxicol. Ind. Health. 1999, 15, 12-25.

59. Rubin, B. S.; Lenkowski, J R.; Schaeberle, C. M.; Vandenberg, L. N.; Ronsheim, P. M.; Soto, A. M. Evidence of altered brain sexual differentiation in mice exposed perinatally to low, environmentally relevant levels of bisphenol A. Endocrinology. 2006, 147, 3681-3691. 
60. Farabollini, F.; Porrini, S.; Della Seta, D.; Bianchi, F.; Dessi-Fulgheri, F. Effects of perinatal exposure to bisphenol A on sociosexual behavior of female and male rats. Environ. Health Perspect. 2002, 110, 409-414.

61. Vandenberg, L. N.; Maffini, M. V.; Wadia, P. R.; Sonnenschein, C.; Rubin, B. S.; Soto, A. M.; Exposure to environmentally relevant doses of the xenoestrogen bisphenol-A alters development of the fetal mouse mammary gland. Endocrinology. 2007, 148, 116-127.

62. Government of Canada Takes Action on Another Chemical of Concern: Bisphenol A- News Release. 2008. http://www.hc-sc.gc.ca/ahc-asc/media/nrcp/ 2008/2008 59-eng.php

63. Bushnik, T.; Haines, D.; Levallois, P.; Levesque, J.; van Oostram, J.; Viau, C. Lead and bisphenol A concentrations in the Canadian population. Health Reports, Component of Statistics Canada, Cataloque no. 82-003-X, 2010.

64. Del Olmo, M.; González-Carado, A.; Navas, N. A., Vilchez, J. L. Determination of bisphenol A in water by gas-chromatography-mass spectrometry. Analytica Chimica Acta. 1997, 346, 87-92.

65. Peltonen, K.; Pukkila, J. Determination of bisphenol A in air by highperformance liquid-chromatography with electrochemical detection. Journal of Chromatography, 1988, 439, 375-380.

66. Ballesteros-Gómez, A.; Rubio, S.; Pérez-Bendito, D. Analytical methods for the determination of bisphenol A in food. Journal of Chromatography A. 2009, 1216, 449-469. 
67. LišKa, I. Fifty years of solid-phase extraction in water analysis- historical development and overview. Journal of Chromatography A. 2000, 885, 3-16

68. Berrueta, L. A.; Gallo, A.; Vicente, F. A review of solid-phase extraction: basic principles and new developments. Chromatographia. 1995, 40 (7), 474-483.

69. Aulakh, J. S.; Malik, A. K.; Kaus, V.; Schmitt-Kopplin, P. A review on solid phase micro extraction- high performance liquid chromatography (SPME-HPLC) analysis of pesticides. Critical Reviews in Analytical Chemistry. 2005, 35, 7185.

70. Lord, H.; Pawliszyn, P. Evolution of solid-phase microextraction technology. Journal of Chromatography A. 2000, 885, 153-193.

71. Risticevic, S.; Niri, V. H.; Vuckovic, D.; Pawliszyn, J. Recent development in solid-phase microextraction. Anal. Bioanal. Chem. 2009, 393, 781-795.

72. Zhang, Z.; Yang, M. J.; Pawlisyn, J. Solid-phase microextraction. Anal. Chem. $1994,66,17,844-853$ A.

73. Ouyang, G.; Pawliszyn, P. SPME in environmental analysis. Anal. Bioanal. Chem. 2006, 386, 1059-1073.

74. Long, R. Q.; Yang, R. L. Carbon nanotubes as superior sorbents for dioxin removal. J. Am. Chem. Soc. 2001, 123, 2058-2059.

75. Zhou, Q.; Xiao, J.; Wang, W.; Using multi-walled carbon nanotubes as solid phase extraction adsorbents to determine dichlorodiphenyltrichloroethane and its 
metabolites at trace level in water samples by high performance liquid chromatography with UV detection. Journal of Chromatography A. 2006, 1125 (2), 152-158.

76. Zhou, Q.; Xiao, J.; Wang, W.; Liu, G.; Shi, Q.; Wang, J. Determination of atrazine and simazine in environmental water samples using multiwalled carbon nanotubes as the adsorbents for preconcentration prior to high performance liquid chromatography with diode array detector. Talanta. 2006, 68 (4), 1309-1315.

77. Katsumata, H.; Matsumoto, T.; Kaneco, S.; Suzuki, T. Ohta, K. Preconcentration of diazinon using multiwalled carbon nanotubes as solid-phase extraction adsorbents. Microchemical Journal. 2008, 88 (1), 82-86.

78. Al-Degs, Y. S.; Al-Ghouti, M. A. Preconcentration and determination of high leachable pesticides residues in water using solid-phase extraction coupled with high-performance liquid chromatography. Journal of Environmental Analytical Chemistry. 2008, 88 (7), 487-498.

79. Cruz-Vera, M.; Lucena R.; Cárdenas, S.; Valcárcel M. Combined use of carbon nanotubes and ionic liquid to improve the determination of antidepressants in urine samples by liquid chromatography. Anal. Bioanal. Chem. 2008, 391, 11391145.

80. Zhao, H.; Wang, L.; Qiu, Y.; Zhou, Z.; Zhong, W.;Li, X. Multiwalled carbon nanotubes as a solid-phase extraction adsorbent for the determination of three barbiturates in pork by ion trap gas chromatography-tandem mass spectrometry 
(GC/MS/MS) following microwave assisted derivatization. Analytica Chimica Acta. $2007,586(1-2), 399-406$.

81. Suárez, B.; Santos, B.; Simonet, B. M.; Cárdenas, S.; Valcárcel, M. Solid-phase extraction-capillary electrophoresis-mass spectrometry for the determination of tetracyclines residues in surface water by using carbon nanotubes as sorbent material. Journal of Chromatography A. 2007, 1175 (1), 127-132.

82. Suárez, B.; Simonet, B. M.; Cárdenas, S.; Valcárcel, M. Determination of nonsteroidal anti-inflammatory drugs in urine by combining an immobilized carboxylated carbon nanotubes minicolumn for solid-phase extraction with capillary electrophoresis-mass spectrometry. Journal of Chromatography A. 2007, 1159 (1-2), 203-207.

83. Cai, Y. Q.; Jiang, G. B.; Liu, J. F.; Zhou, Q. K. Multi-walled carbon nanotubes packed cartridge for the solid-phase extraction of several phthalate esters from water samples and their determination by high performance liquid chromatography. Analytica Chimica Acta. 2003, 494 (1-2), 149-156.

84. Niu, H. Y.; Cai, Y. Q.; Shi, Y. L.; Wei, F. S.; Liu, J. M.; Jiang, G. B. A new solid-phase extraction disk based on a sheet of single-walled carbon nanotubes. Anal. Bioanal. Chem. 2008, 392, 927-935.

85. Salam, M. A.; Burk, R. Solid phase extraction of polyhalogenated pollutants from freshwater using chemically modified multi-walled carbon nanotubes and 
their determination by gas chromatography. Journal of Separation Science. 2009, $32(7), 1060-1068$.

86. Liang, P.; Liu, Y.; Guo, L.; Zeng, J.; Lu, H. Multiwalled carbon nanotubes as solid-phase extraction adsorbent for the preconcentration of trace metal ions and their determination by inductively coupled plasma atomic emission spectrometry. J. Anal. At. Spectrom. 2004, 19, 1489, 1492.

87. Liang, P.; Liu, Y.; Guo, L. Determination of trace rare earth elements by inductively coupled plasma atomic emission spectrometry after preconcentration with multiwalled carbon nanotubes. Spectrochimica Acta Part B: Atomic Spectroscopy. 2005, 60 (1), 125-129.

88. Barbosa, A. F.; Segatelli, M. G.; Pereira, A. C.; de Santana Santos, A.; Kubota, L. T.; Luccas, P. O.; Tarley, C. R. T. Solid-phase extraction system for Pb (II) ions enrichment based on multiwall carbon nanotubes coupled on-line to flame atomic absorption spectrometry. Talanta. 2007, 71 (4), 1512-1519.

89. Shang, X. H. Flow injection on-line solid phase extraction using multi-walled carbon nanotubes as sorbent for cold vapor atomic fluorescence spectrometric determination of trace mercury in water samples. Atomic Spectroscopy. 2007, $28(1), 35-40$.

90. Afzali, D.; Mostafavi, A. Potential of Modified Multiwalled Carbon Nanotubes with 1-(2-Pyridylazo)-2-naphtol as a New Solid Sorbent for the Preconcentration 
of Trace Amounts of Cobalt(II) Ion. Analytical Sciences. 2008, 24 (9), 11351139.

91. Tuzen, M.; Soylak, M. Multiwalled carbon nanotubes for speciation of chromium in environmental samples. Journal of Hazardous Materials. 2007, 147 (1-2). 219225.

92. Ravelo, L. M.; Herrera-Herrera, A. V.; Hernández-Borges, J.; RodríguezDelgado, M. A.; Carbon nanotubes: Solid-phase extraction. Journal of Chromatography A. 2010, 1217 (16), 2618-2641.

93. Cai, Y.; Jiang, G.; Liu, J. F.; Zhou, Q. Multiwalled carbon nanotubes as a solidphase extraction adsorbent for the determination of bisphenol A, 4-n-nonylphenol and 4-tert-octylphenol. Anal. Chem. 2003, 75, 10, 2517-2521.

94. Kuo, C. Y. Comparison with as-grown and microwave modified carbon nanotubes to removal aqueous bisphenol A. Desalination. 2009, 249, 976-982.

95. Rastkari, N.; Ahmadkhaniha, R.; Yunesian, M.; Baleh, L. J.; Mesdaghinia, A. Sensitive determination of bisphenol $\mathrm{A}$ and bisphenol $\mathrm{F}$ in canned food using a solid-phase microextraction fibre with single-walled carbon nanotubes before GC/MS. Food Additives and Contaminants. 2010, 27, 10, 1460-1468.

96. Li, Q.; Wang, X.; Yuan, D. Preparation of solid-phase microextraction fiber coated with single-walled carbon nanotubes by electrophoretic deposition and its 
application in extracting phenols from aqueous samples. J. Chromato. A. 2009, $1216,1305-1311$.

97. Basheer, C.; Alnedhary, A. A.; Rao, M.; Vallinyaveettil, S.; Lee, H. K. Development and application of porous membrane -protected carbon nanotube micro-solid-phase extraction combined with gas chromatography/mass spectrometry. Anal. Chem. 2006, 78, 2853-2858.

98. Basheer, C.; Chong, H. G.; Hii, T. M.; Lee, H. K. Application of porous membrane-protected micro-solid-phase extraction combined with HPLC for the analysis of acidic drugs. Anal. Chem. 2007, 79, 6845-6850.

99. Salam, M. A.; Burk, R. Novel application of modified multiwalled carbon nanotubes as a solid phase extraction adsorbent for the determination of polyhalogenated organic pollutants in aqueous solution. Anal. Bioanal. Chem. $2008,390,2159-2170$.

100. Salam, M. A. Chemical modification of multi-walled carbon nanotubes and their potential applications as new adsorbents for solid phase extraction from aqueous solution. Applied Surface Science, 2008, 255 (5), 1975-1981.

101. Bagheri, H.; Saraji, M. Conductive polymers as new media for solid-phase extraction: Isolation of chlorophenols from water sample. Journal of Chromatography A. 2003, 986 (1), 111-119.

102. Sarafraz-Yazdi, A.; Amiri, A. Liquid-phase microextraction. Trends in Analytical Chemistry. 2010, 29, 1, 1-14. 
103. Chakrapani, N.; Zhang, Y. M.; Nayak, S. K.; Moore, J. A.; Carroll, D. L.; CHoi, Y. Y.; Ajayan, P. M.; Chemisorption of Acetone on Carbon Nanotubes. J. Phys. Chem. B. 2007, 107, 9308-9311.

104. Tang, Z. R.; The adsorption of methanol at the defective site of a singlewalled carbon nanotube. Physica B, 2010, 405, 770-773.

105. Krishnan, A. V.; Stathis, P.; Permuth, S. F.; Tokes, L.; Feldman, D. Bisphenol A: an estrogenic substance is released from polycarbonate flasks into autoclaving. Endocrinology. 1993, 132, 2279-2286.

106. Barber, A. H.; Cohen, S. R.; Wagner, H. D.; Static and dynamic wetting measurements of single carbon nanotubes. Physical Review Letters. 2004, 92, $18,186103-1-186103-4$

107. Pan, B.; Lin. D.; Mashayekhi, H.; Xing, B. Adsorption and hysteresis of bisphenol A and $17 \alpha$-ethinyl estradiol on carbon nanomaterials. Environ. Sci. Technol. 2008, 42, 5480-5485. 


\section{APPENDIX A}

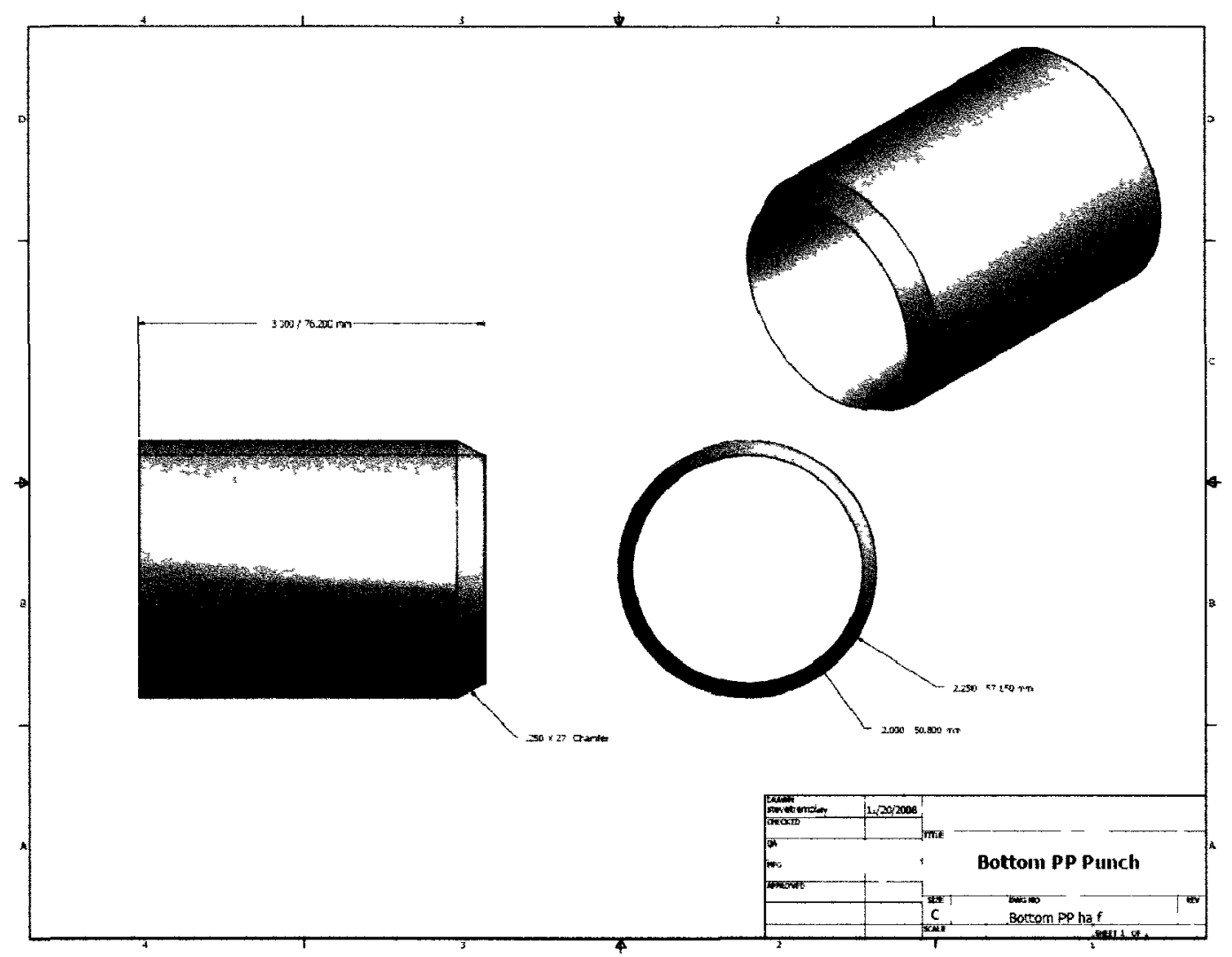

A. 1 


$$
\begin{array}{r}
=0 \\
=0
\end{array}
$$




$$
\begin{aligned}
& 10^{\circ} \\
& 10^{\circ}
\end{aligned}
$$



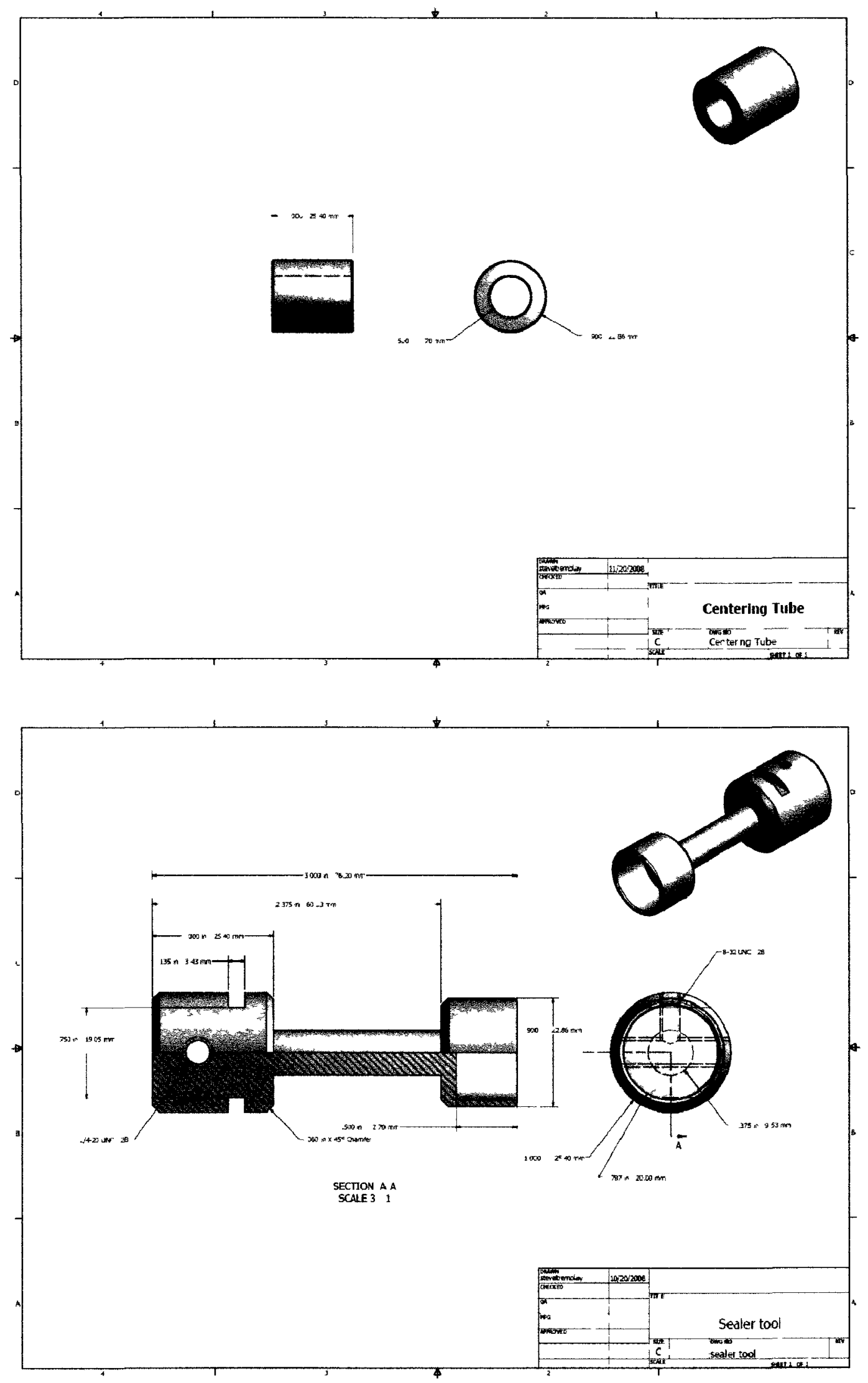

A. 4 

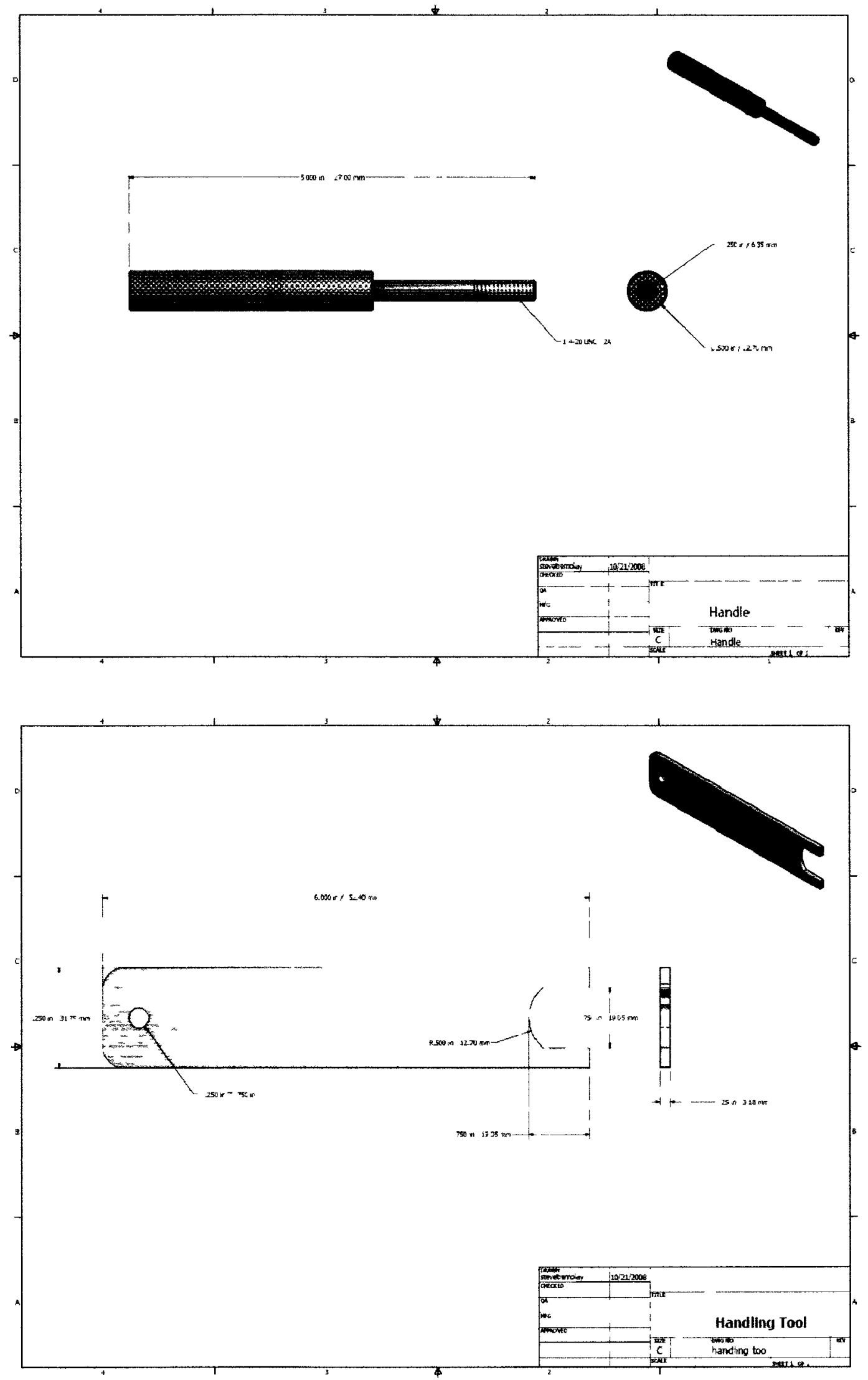

A. 5 